\title{
A Coupled Tire Structure-Acoustic Cavity Model
}

\author{
By \\ Leonardo Molisani \\ Dissertation submitted to the Faculty of the \\ Virginia Polytechnic Institute and State University \\ in Partial fulfillment of the requirements for the degree of \\ Doctor of Philosophy \\ in \\ Mechanical Engineering
}

Committee Members:

Dr. Ricardo A. Burdisso, Chairman

$\begin{array}{ll}\text { Dr. Martin Klaus } & \text { Dr. Alfred L. Wicks } \\ \text { Dr. Scott Hendricks } & \text { Dr. Marty Johnson }\end{array}$

Dr. Sergio Preidikman

May 7, 2004

Blacksburg, Virginia

The United States of America

Keywords: acoustic cavity resonances, acoustic cavity-tire structure interaction, resonance control techniques.

Copyright $@ 2004$, Leonardo Molisani 


\title{
A Coupled Tire Structure-Acoustic Cavity Model
}

\author{
by \\ Leonardo Molisani \\ (ABSTRACT) \\ Dr. Ricardo A. Burdisso, Chairman \\ Department of Mechanical Engineering \\ Virginia Polytechnic Institute \& State University
}

Recent experimental results have shown that the vibration induced by the tire air cavity resonance is transmitted into the vehicle cabin and may be responsible for significant interior noise. The tire acoustic cavity is excited by the road surface through the contact patch on the rotating tire. The effect of the cavity resonance is that results in significant forces developed at the vehicle's spindle, which in turn drives the vehicle's interior acoustic field. This tire-cavity interaction phenomenon is analytically investigated by modeling the fully coupled tire-cavity systems. The tire is modeled as an annular shell structure in contact with the road surface. The rotating contact patch is used as a forcing function in the coupled tire-cavity governing equation of motion. The contact patch is defined as a prescribed deformation that in turn is expanded in its Fourier components. The response of the tire is then separated into static (i.e. static deformation induced by the contact patch) and dynamic components due to inertial effects. The coupled system of equations is solved analytically in order to obtain the tire acoustic and structural responses. The model provides valuable physical insight into the patch-tire-acoustic interaction phenomenon. The influence of the acoustic cavity resonance on the spindles forces is shown to be very important. Therefore, the tire cavity resonance effect must be reduced in order to control the tire contribution to the vehicle interior. The analysis and modeling of two feasible approaches to control the tire acoustic cavity resonances are proposed and investigated. The first approach is the incorporation of secondary acoustic 
cavities to detune and damp out the main tire cavity resonance. The second approach is the addition of damping directly into the tire cavity. The techniques presented in this dissertation to suppress the adverse effects of the acoustic cavity in the tire response, i.e. forces at the spindle, show to be very effective and can be easily applied in practice. 


\section{TABLE OF CONTENTS}

TABLE OF CONTENTS .......................................................... iv

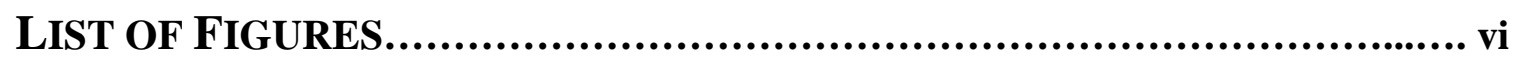

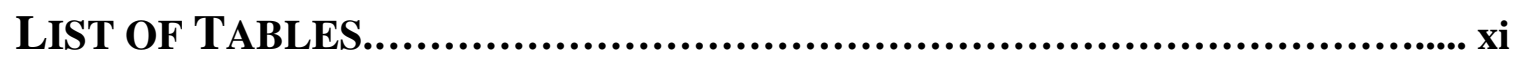

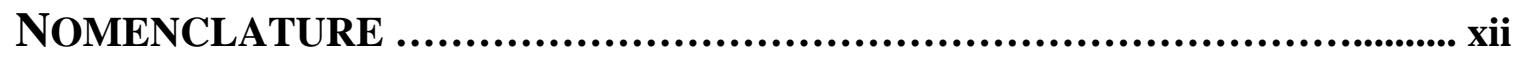

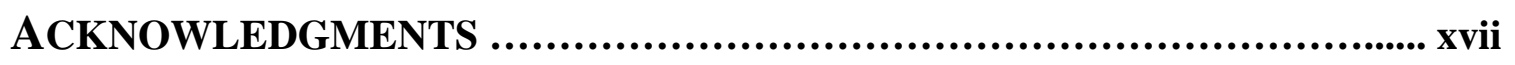

CHAPTER 1: INTRODUCTION ............................................... 1

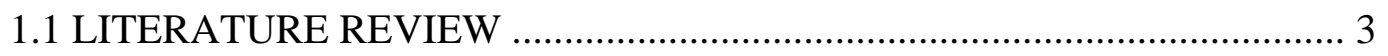

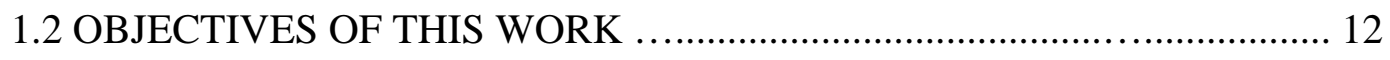

1.3 FOREMOST ORIGINAL CONTRIBUTIONS OF THIS WORK .................13

1.4 DISSERTATION ORGANIZATION .......................................................... 14

CHAPTER 2: STRUCTURAL MODEL ....................................... 16

2.1 FREE TIRE MODEL …….................................................................. 17

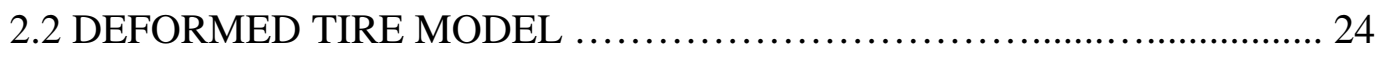

2.3 FORCES TRANSMITTED TO THE "SPINDLE” ........................................ 38

2.4 FREE TIRE NUMERICAL SIMULATIONS ............................................... 45

2.5 DEFORMED TIRE NUMERICAL SIMULATIONS .................................... 51

CHAPTER 3: COUPLED TIRE MODEL ........................................... 56

3.1 THE INTERIOR ACOUSTIC PROBLEM …............................. 56

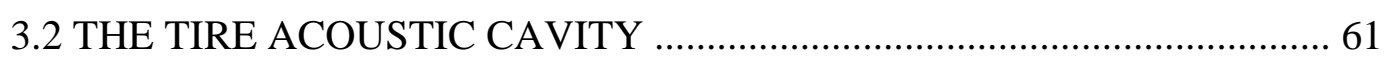

3.3 COUPLED STRUCTURAL-ACOUSTIC PROBLEM ………….................. 69

3.4 FREE TIRE NUMERICAL SIMULATIONS ............................................ 75

3.5 DEFORMED TIRE NUMERICAL SIMULATIONS ................................... 81

\section{CHAPTER 4: CONTROL OF THE TIRE ACOUSTIC CAVITY}

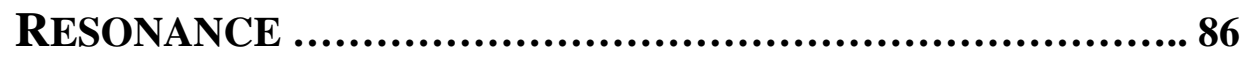

4.1 SECONDARY CAVITIES CONTROL APPROACH ........................... 87 
4.2 NUMERICAL SIMULATIONS OF SECONDARY CAVITIES

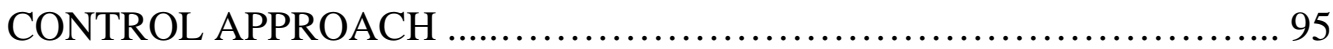

4.3 DAMPING APPROACH USING VISCOELASTIC SCREENS ............ 114

4.4 VISCOELASTIC SCREENS CONTROL APPROACH .................. 120

CHAPTER 5: SYNOPSIS, CONCLUSIONS, AND RECOMMENDATIONS

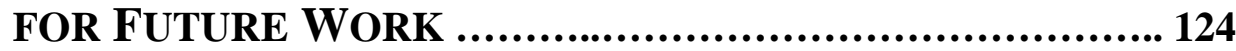

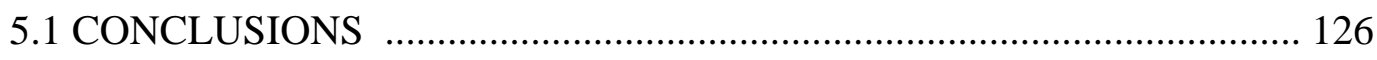

5.2 RECOMMENDATIONS FOR FUTURE WORK ........................ 127

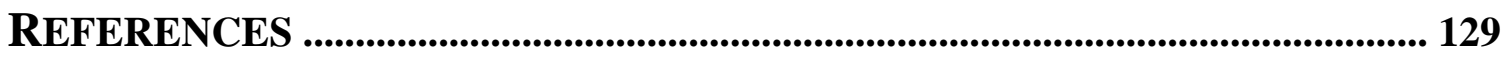

APPENDIX A: TIRE SIZE NOMENCLATURE .............................. 134

APPENDIX B: SOLVING THE CONTACT PATCH EQUATIONS ........... 137

APPENDIX C: GENERAL BOUNDARY FORCES FOR A SHELL ........... 140

APPENDIX D: SOLVING THE SPECTRAL PROPERTIES OF THE

HELMHOLTZ OPERATOR ........................................................ 147

APPENDIX E: THE COUPLING MATRIX ............................................................. 151

APPENDIX F: SCREEN ACOUSTIC IMPEDANCE MODEL ............................ 154

APPENDIX G: SOLVING THE COUPLING MATRICES .................................... 157

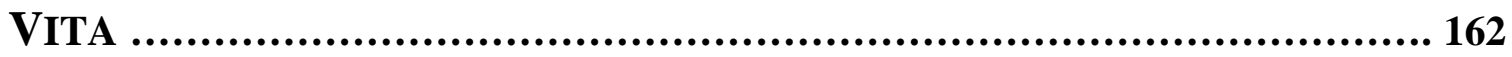




\section{LIST OF FIGURES}

Figure 1.1: Evolution of vehicle noise sources and control technologies .......................... 2

Figure 1.2: Vertical acceleration level at the spindle in a coasting test .............................. 4

Figure 1.3: Effects of filling the tire cavity with polyurethane foam on

the sound pressure level at the driver place in a coasting test ........................ 5

Figure 1.4: Dominating modes of the tire cavity resonance …………............................ 6

Figure 1.5: (a) Un-deformed or Free Tire model, (b) Deflected tire model ....................... 7

Figure 1.6: Dynamic Stiffness comparison ...................................... 8

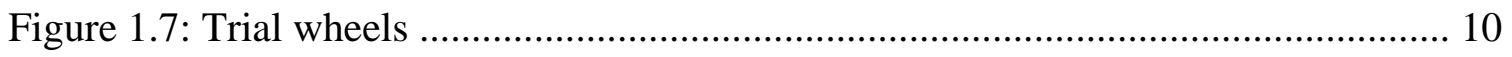

Figure 1.8: Approach to control the cavity effects ........................................................... 11

Figure 2.1: Simplified tire model ........................................................................... 18

Figure 2.2: Radial nodal patters for circular cylindrical shells ........................................ 21

Figure 2.3: Schematic of the contact patch modeling ................................. 25

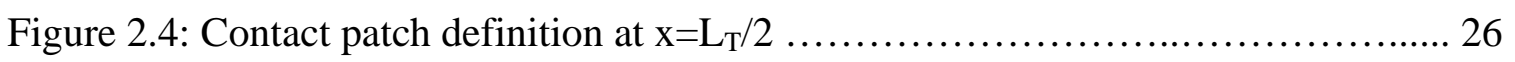

Figure 2.5: Prescribed deformation function $d\left(\frac{L_{T}}{2}, \theta\right)$ for a

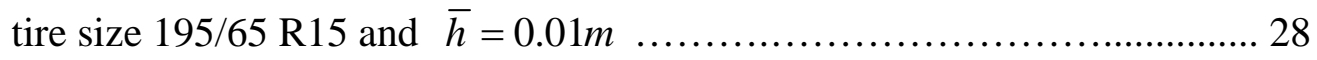

Figure 2.6: Radial displacement due to prescribed deformation at $x=\frac{L_{T}}{2}$

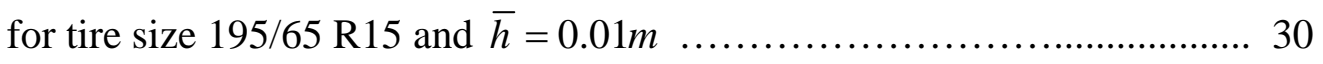

Figure 2.7: Radial displacement field $w^{S}[\mathrm{~m}]$ for tire size 195/65 R15 with

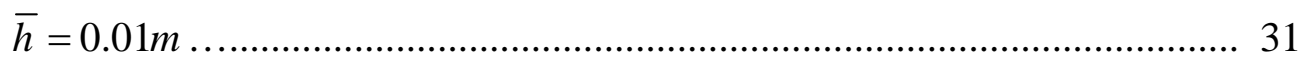

Figure 2.8: Radial displacement comparison between model and experimental data for tire size 195/65 R15 .............................. 32

Figure 2.9: Spindle force and moment .............................................. 38

Figure 2.10: Structural Modal Amplitude for mode $\Phi_{14}^{(1)}$

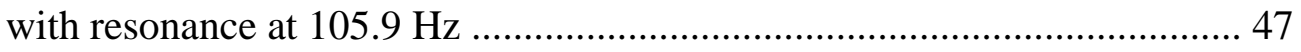

Figure 2.11: Vertical force $\mathrm{F}_{\mathrm{z}}$ at the spindle .............................................................. 48 
Figure 2.12: Horizontal moment $\mathrm{M}_{\mathrm{y}}$ at the spindle

Figure 2.13: Radial displacement for the in vacuo free tire model at the external force location

Figure 2.14: Point accelerance for the in vacuo free tire at the location of the external force. Acoustic cavity resonance not included in the model

Figure 2.15: Structural Modal Amplitude for mode $\Phi_{14}^{(1)}$ with resonance at

105.9 $\mathrm{Hz}$ for the deformed tire model 51

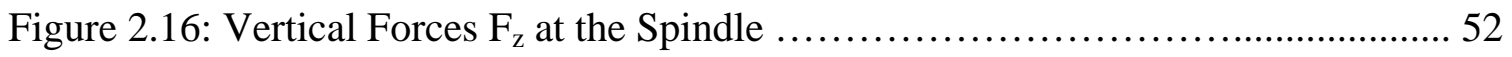

Figure 2.17: Horizontal Moment $\mathrm{M}_{\mathrm{y}}$ at the Spindle 53

Figure 2.18: Radial displacement for the in vacuo deformed tire model at

$$
\text { location }\left(x=\frac{L_{T}}{2}, \theta=0\right)
$$

Figure 2.19: Point accelerance for the in vacuo deformed tire model at

$$
\text { location }\left(x=\frac{L_{T}}{2}, \theta=0\right) \text {. }
$$

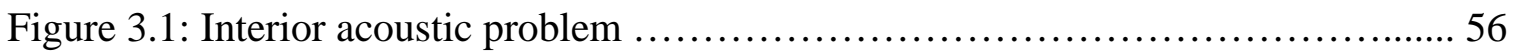

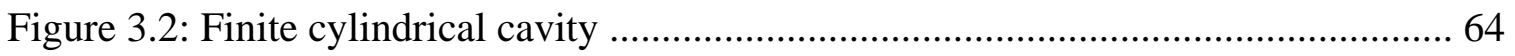

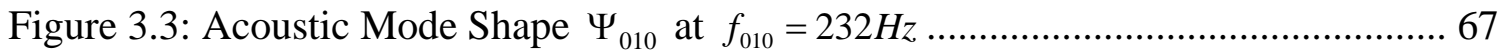

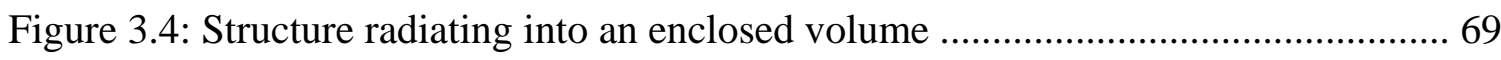

Figure 3.5: Dynamic interaction between the structure and the fluid ........................... 70

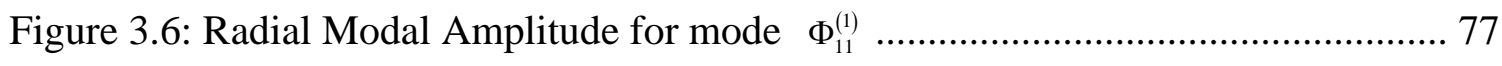

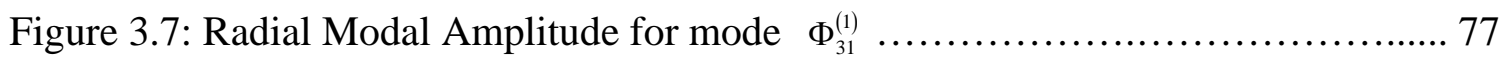

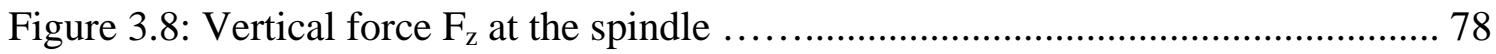

Figure 3.9: Horizontal moment $\mathrm{M}_{\mathrm{y}}$ at the spindle................................................... 79

Figure 3.10: Radial displacement for the free tire model at the

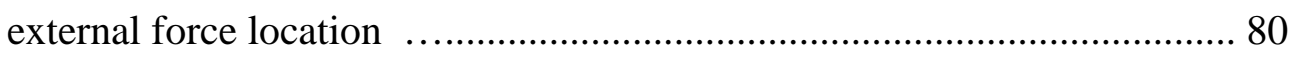

Figure 3.11 Point accelerance for the free tire model at the external force location ....... 80

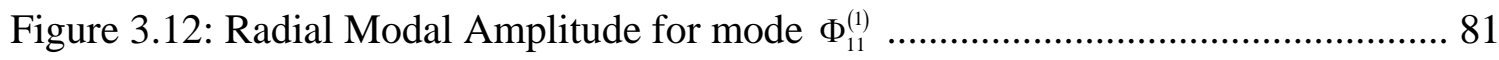

Figure 3.13: Radial Modal Amplitude for mode $\Phi_{13}^{(1)} \quad$................................................ 82 


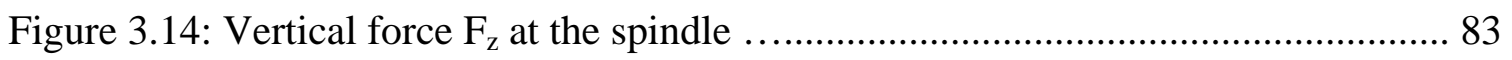

Figure 3.15: Horizontal moment $\mathrm{M}_{\mathrm{y}}$ at the spindle ........................................................ 83

Figure 3.16: Radial displacement for the deformed tire model at

$$
\text { location }\left(x=\frac{L_{T}}{2}, \theta=0\right) \text {. }
$$

Figure 3.17: Point accelerance for the deformed tire model at

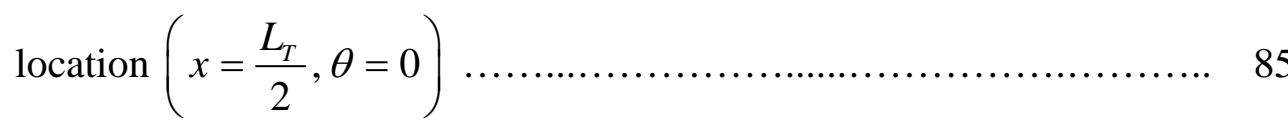

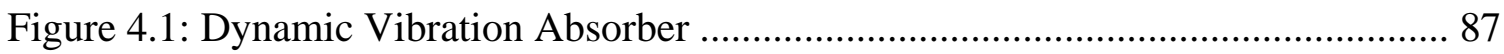

Figure 4.2: Tire and secondary cavities acoustic modeling ............................................ 89

Figure 4.3: Schematic of the acoustic model and coupling interface ............................. 91

Figure 4.4: Magnitude of the vertical force at the spindle for different secondary cavity length for the free tire model .............................. 97

Figure 4.5: Magnitude of the horizontal moment at the spindle for different secondary cavity length for the free tire model ............................................. 98

Figure 4.6: Magnitude of the vertical force at the spindle for different secondary cavity radius for the free tire model ................................................ 99

Figure 4.7: Magnitude of the horizontal moment at the spindle for different secondary cavity radius for the free tire model

Figure 4.8: Magnitude of the vertical force at the spindle for different screen impedance for the free tire model 101

Figure 4.9: Magnitude of the horizontal moment at the spindle for different screen impedance for the free tire model ...................................................... 102

Figure 4.10: Magnitude of the point accelerance for the free tire model ....................... 103

Figure 4.11: Magnitude of the vertical force at the spindle for different secondary cavity azimuth position for the free tire model

Figure 4.12: Magnitude of the horizontal moment at the spindle for different secondary cavity azimuth position for the free tire model

Figure 4.13: Magnitude of the vertical force at the spindle for the deformed tire model 106 
Figure 4.14: Magnitude of the horizontal moment at the spindle for the deformed tire model 107

Figure 4.15: Magnitude of the vertical force at the spindle for the deformed tire model 108

Figure 4.16: Magnitude of the horizontal moment at the spindle for the deformed tire model 109

Figure 4.17: Magnitude of the vertical force at the spindle for the deformed tire model 110

Figure 4.18: Magnitude of the horizontal moment at the spindle for the deformed tire model

Figure 4.19: Magnitude of the point accelerance 112

Figure 4.20: Magnitude of the vertical force at the spindle for different secondary cavity azimuth position

Figure 4.21: Magnitude of the horizontal moment at the spindle for different secondary cavity azimuth position

Figure 4.22: Hold and viscoelastic screens arranged in the tangential direction 114

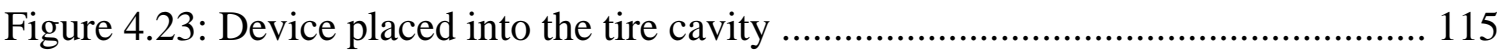

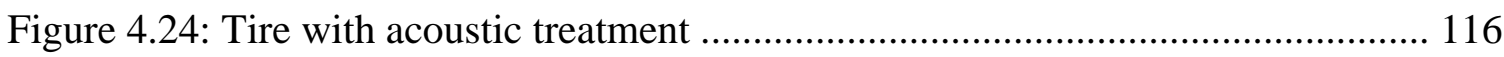

Figure 4.25: View of the viscoelastic screens inside of the main cavity ....................... 118

Figure 4.26: Experiment to validate the concept of the viscoelastic screens ................. 119

Figure 4.27: FRF Magnitudes with 8 Screens - 24 Slices (blue line)

and without screens (red line) at Microphone 3

Figure 4.28: Magnitude of the vertical force at the spindle 121

Figure 4.29: Magnitude of the horizontal moment at the spindle 121

Figure 4.30: Magnitude of the vertical force at the spindle 122

Figure 4.31: Magnitude of the horizontal moment at the spindle 123

Figure A.1: Schematic tyre dimension 134

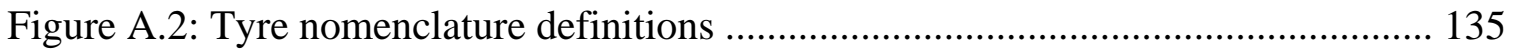

Figure C.1: Stresses in shell coordinates .................................... 140

Figure C.2: Force resultants in shell coordinates 142 
Figure C.3: Moment resultants in shell coordinates ................................................ 143

Figure F.1: Facesheet Normalized Impedance - Resistive Component ........................ 155

Figure F.2: Facesheet Normalized Impedance - Reactive Component ......................... 156 


\section{LIST OF TABLES}

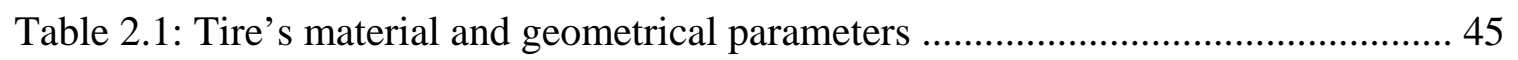

Table 2.2: Tire structure natural frequencies between 0 and $400 \mathrm{~Hz}$......................... 46

Table 3.1: Description of the effect of axial variation on the coupling between structural and acoustic modes of same azimuth variation ......................... 74

Table 3.2: Description of the effect of in the circumferential direction on the coupling between structural and acoustic modes .................................... 74

Table 3.3: Tire acoustic cavity natural frequencies for tire 195/65 R15

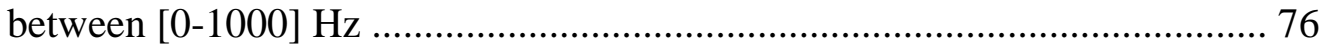

Table 4.1: Acoustic damping at 2nd resonance and expected noise

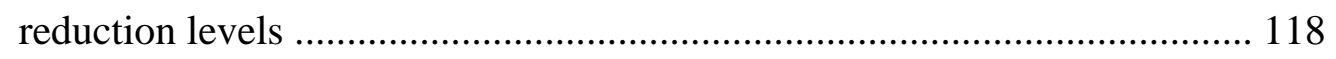

Table A.1: Tire’s material and geometrical parameters ........................................... 136 


\section{NOMENCLATURE}

$a$

$A_{m n}^{(j)}$

$b$

$B(\underset{\sim}{r})$

$\tilde{B}_{q p l}$

C

$\hat{c}$

$d$

$d(x, \theta)$

E

$E_{c}$

$E[d B]$

$f_{q p l}$

$\left\{f_{u}, f_{v}, f_{w}\right\}^{\mathrm{T}}$

$\left\{f_{m n}\right\}$

$\{F\}$

$F_{y}$

$F_{z}$

$\{F\}_{\text {Left } x=0}$

$\{F\}_{\text {Rigth } x=L_{T}}$

$\vec{F}_{\alpha}, \vec{F}_{\beta}$

$F(\underset{\sim}{r})$

$\{F\}_{\text {Spindle }}$
Tire outer radius

Modal amplitudes

Tire inner radius

Boundary condition operator

Coefficient of the Green’s function series expansion

Speed of sound

Complex speed of sound $\hat{c}=c\left(1+i \eta_{A}\right)$

Spindle length

Prescribed deformation function

Young's modulus of the tire material

Complex Young's modulus of the tire material defined as $E\left(1+i \eta_{\text {Shell }}\right)$

Potential noise level reduction in $[d B]$

Acoustic Natural Frequency in Hertz

Axial, tangential and radial force components

Generalized force vector

Forces per unit length vector in Cartesian components given by $\left\{F_{x^{\prime}}, F_{y^{\prime}}, 0\right\}$

Force per unit length component of $\{F\}$ in the horizontal direction

Force per unit length component of $\{F\}$ in the vertical direction

Left side edge force per unit length at the spindle

Right side edge force per unit length at the spindle

Reaction Forces at the boundary $\alpha$ and $\beta$ respectively

Distribution of volume velocity source strength per unit volume

Force per unit length at the spindle 
$\{F\}_{\text {spindle }}^{\text {Total }} \quad$ Resultant force at the spindle

$G\left(\underset{\sim}{r}||_{\sim}\right) \quad$ Green’s Function

$G\left(x, \theta, r \mid x_{o}, \theta_{o}, r_{o}\right)$ Green's function associated to the tire acoustic cavity domain

$G_{j}\left(\eta \mid \eta_{o}\right) \quad$ Green's function associated to the $j^{\text {th }}$ secondary cavity

$\hat{G}\left[x, \theta, a \mid\left(x_{o}, x_{o}^{(1)}, x_{o}^{(2)}\right),\left(\theta_{o}, \theta_{o}^{(1)}, \theta_{o}^{(2)}\right),(a, b, b)\right]$ Modified Green’s function

$h_{\text {Shell }} \quad$ Thickness of the tire structure

$\bar{h} \quad$ Contact patch depth

$h(t) \quad$ Harmonic component of the contact patch $h(t)=\bar{h}+\varepsilon e^{i \omega t}$

$\left[H_{s r}\right] \quad$ Structural transfer function matrix

i Imaginary unit $i=\sqrt{-1}$

[I] Identity matrix

$J_{p}(\cdot) \quad$ Bessel's Function of order "p" of the First Kind

$k \quad$ Free Field Acoustic Wavenumber

$k_{i}^{(j)} \quad$ Eigenvalue or Acoustic wavenumber of the $j^{\text {th }}$ secondary cavity

$k_{q p l} \quad$ Acoustic Wavenumber or Linear Wave Operator eigenvalue

$K=\frac{h_{\text {Shell }}^{2}}{12 a^{2}} \quad$ Nondimensional Tire Thickness Parameter

$K_{z z} \quad$ Dynamic Stiffness in the z-direction

$\left[K_{m n}\right] \quad$ Stiffness matrix

$K_{x}, K_{\theta}, \tau \quad$ Change in curvatures and twist of the shell middle surface

L Integral operator

$L_{T} \quad$ Tire width

$\underset{\sim}{L} \quad$ Adjoint Integral operator of $L$

$\left[L_{c}\right] \quad$ Shell operator associated to the Donnell-Mushtari-Vlasov theory

$\left[M_{m n}\right] \quad$ Mass matrix

$\vec{M}_{\alpha}, \vec{M}_{\beta} \quad$ Reaction Moments at the boundary $\alpha$ and $\beta$ respectively 
$M_{\alpha}, M_{\alpha \beta} \quad$ Resultant moments at shell edge $\beta$ constant

$M_{\beta}, M_{\beta \alpha} \quad$ Resultant moments at shell edge $\alpha$ constant

$M_{x}, M_{\theta}, M_{x \theta}$ Resultant moments at the boundary

$\{M\}_{\text {Spindle }} \quad$ Moment per unit length at the spindle

$\{M\}_{\text {Spindle }}^{\text {Total }} \quad$ Resultant moment at the spindle

$N_{\alpha}, N_{\alpha \beta}$ and $Q_{\alpha} \quad$ Resultant forces at shell edge $\beta$ constant

$N_{\beta}, N_{\beta \alpha}$ and $Q_{\beta} \quad$ Resultant forces at shell edge $\alpha$ constant

$N_{x}, N_{\theta}, N_{x \theta}$ Resultant forces at the boundary

$p(\underset{\sim}{r}) \quad$ Acoustic pressure

$p_{1}(\eta) \quad$ Acoustic pressure of the first secondary cavity

$p_{2}(\eta) \quad$ Acoustic pressure of the second secondary cavity

$Q_{x}, Q_{\theta} \quad$ Shear force components

$\left\{r^{s-L e f t}\right\} \quad$ Distance from left shell edge to the spindle

$\left\{r^{s-R i g h t}\right\} \quad$ Distance from right shell edge to the spindle

$[R] \quad$ Rotation matrix

$S_{x} \quad$ Tangential force per unit length component of $\left\{F_{x}\right\}$

$T_{x} \quad$ Normal force per unit length component of $\left\{F_{x}\right\}$

$\vec{u} \quad$ Displacement vector

$\left\{u_{i}\right\} \quad$ Displacement vector

$\{u, v, w\}^{\mathrm{T}} \quad$ Axial $(u)$, tangential $(v)$, and radial $(w)$ displacement components

$\left\{u^{t}\right\} \quad$ Total displacement vector given by $\left\{u^{t}, v^{t}, w^{t}\right\}^{T}$

$\left\{\tilde{u}^{s}, \tilde{v}^{s}, \tilde{w}^{s}\right\}^{T} \quad$ Displacement components around the equilibrium position $\bar{h}$

$\dot{w} \quad$ Radial velocity component

$W_{i} \quad$ Virtual work done by the reaction forces at the $i^{\text {th }}$ shell boundary

$W_{m n}^{(3)} \quad$ Radial coefficient of the series approximating radial displacement 
$\bar{W}_{m n}^{(3)} \quad$ Radial coefficient associated to static deformation

$\left\{\tilde{W}_{s n}^{(3)}\right\} \quad$ Radial coefficient associated to kinematics deformation

$x_{o}^{(1)}, \theta_{o}^{(1)} \quad$ Position of the first secondary cavity in the inner shell

$x_{o}^{(2)}, \theta_{o}^{(2)} \quad$ Position of the second secondary cavity the inner shell

$Y_{p}(\cdot)^{1} \quad$ Bessel's Function of order " $p$ ” of the Second Kind

$\dot{z}_{j} \quad$ Particle velocity at the interface of the $j^{\text {th }}$ secondary cavity

$Z_{S}^{(j)} \quad$ Acoustic impedance associated to the $j^{\text {th }}$ secondary cavity

$\sum_{q=0}^{Q} \sum_{p=0}^{P} \sum_{l=0}^{L}\left[\alpha_{\text {srmnqpl }}\right] \quad$ Fluid coupling matrix

$\sum_{q=0}^{Q} \sum_{p=0}^{P} \sum_{l=0}^{L}\left[\alpha_{\text {srmnqpl }}^{(1)}\right] \quad$ Coupling Matrix due to the interaction of the first secondary cavity

$\sum_{q=0}^{Q} \sum_{p=0}^{P} \sum_{l=0}^{L}\left[\alpha_{\text {srmnqpl }}^{(2)}\right] \quad$ Coupling Matrix due to the interaction of the second secondary cavity

$\delta\left(\underset{\sim}{r}-\sim_{\sim}\right) \quad$ Dirac delta

$\delta_{q r} \quad$ Kronecker delta defined $\delta_{q r}=\{0$ for $q \neq r$, or 1 for $q=r\}$

$\varepsilon \quad$ Linearization parameter $\varepsilon \ll \bar{h}$

$\varepsilon_{x}, \varepsilon_{\theta}, \varepsilon_{x \theta} \quad$ Strain components

$\varepsilon_{\mathfrak{J}} \quad$ Integration constant related to the Dirac delta

$\eta \quad$ Secondary cavity local coordinate

$\eta_{A} \quad$ Acoustic loss factor

$\eta_{\text {Shell }} \quad$ Tire material loss factor

$\theta_{p} \quad$ Boundary of the contact patch in the azimuth direction

$\lambda_{m} \quad$ Coupling structural modes parameter

$\Lambda_{q p l} \quad$ Normalization factor of the Acoustic Mode Shapes

$v \quad$ Poison ratio of the tire material

$\rho \quad$ Density of the tire material

${ }^{1}$ If $p$ is integer the function is known as a Newmann's Function. 


$\begin{array}{ll}\rho_{A} & \text { Air density } \\ {\left[\Phi_{m n}\right]} & \text { Modal matrix } \\ \chi_{i}^{(j)}(\eta) & \text { Eigenfunction or Acoustic mode shape of the } j^{\text {th }} \text { secondary cavity } \\ \Psi_{q p l}(r, \theta, x) & \text { Eigenfunction or Acoustic Mode Shape in cylindrical coordinates } \\ \omega & \text { Angular frequency } \\ \omega_{q p l} & \text { Angular Natural frequency } \\ \Omega & \text { Normalized frequency } \\ \vec{\Omega} & \text { Rotation vector } \\ \frac{1}{R_{\alpha}} & \text { Curvatures of the } \alpha \text { curves } \\ \frac{1}{R_{\beta}} & \text { Curvatures of the } \beta \text { curves } \\ \Im & \text { Domain of the operator } L \\ \nabla^{2} & \text { Bilaplacian Operator } \\ \square(\cdot)=\nabla^{2}(\cdot)-\frac{1}{c^{2}} \frac{d^{2}(\cdot)}{d t^{2}} & \text { Linear Wave Operator }\end{array}$




\section{ACKNOWLEDGMENTS}

I will like to express my gratitude to my advisor Dr. Ricardo Burdisso for his guidance, assistance, and understanding. Dr. Burdisso guidance contributes not only in this work but also in great part in my education since these few years in Virginia Tech. Dr. Burdisso assistance with the precise intuition based in his vast knowledge was fundamental to bring clarity in the moment of obscurity in the projects that I have the opportunity to work with him. Dr. Burdisso understanding played a very important role when the human being part had to be considered shown his outstanding quality as a person.

I also like to thank to Dr. Martin Klaus for his encouragement and support in opening the door to the world of the Mathematics.

My sincere admiration and gratitude to Dr. Sergio Preidikman for accept to be part in my committee. I worked in the past with Dr. Preidikman back in the National University of Río Cuarto for a short period of time but enough to admire his knowledge in the scientific area of mutual interest and his quality as a being.

I also whish to thank Dr. Alfred L. Wicks, Dr. Scott Hendricks, and Dr. Marty Johnson for serving in my committee and offering their help in the development of this work.

I am grateful to the staff of the Noise and Vibration Team from Michelin North America, Inc. for the financial support in part of this work. Special thank to Dr. Dimitri Tsihlas from Michelin North America, Inc. for his encouragement and appreciation for the associated research project. I also want to thank the collaboration of Hiroshi Yamauchi from Dynamics Testing Vehicle from Mitsubishi Motors Corporation for his contribution with experimental data and his interest related to this work. I am grateful to the members of the VAL staff Lynda C. King and Dawn Williams-Bennett and the Mechanical 
Engineering staff Eloise McCoy, Cathy Hill, Ben Poe and Jamie Archual for their help and support.

I will like to express my appreciation for their moral support and friendship during these years to Dr. Raúl Andruet and Aida Mendez Delgado, Nikolaos Georgopoulos, Patricio Ravetta, Juan Carlos del Real Romero, Diego de la Riva, Tatiana and Virgilio Centeno, Dione and Roberto Cordero, Valery and Edwin Robinson, Cathia Frago, and Jason M. Anderson.

I am thankful to Santiago Alonso for his friendship and company in the looking for answer to the wonder of linear operator theory. I also want to express my gratitude to Professor Rodolfo Duelli for his constant back-up. I am particularly appreciative to Professor Diego Moitre of the Engineering Department of the National University of Río Cuarto. I would like also to express my most sincere gratitude to Mariano, Ricardo, Rafael, Gabriel, Luis, and Etelvina for their continuing friendship and moral support upon these years.

I am also appreciative of the encouragement and support of my mother Noemí, my father Luis, and my sister Carina. I want to state my gratitude to my uncle Oscar for his constant encouragement toward to science.

I want to express deep appreciation to Beatriz for her unconditionally patient, support, and courage to came from a far away place to be with me.

Finally, I want to express my love and gratitude to María Paula my daughter and my future. Because of her love and endurance residing back in Argentina this work is possible. 


\section{Dedicated to MARÍA PAULA}




\section{CHAPTER 1: INTRODUCTION}

I

n motorized vehicles there are many different noise sources. Vehicle noise can be annoying and stressful to drivers and, consequently, consumers continuously demand quieter automobiles. The industry responds to this need with the development of new technologies to reduce vehicle noise. Therefore, noise reduction methods implemented over the last 30 years had led to a gradual transformation of the dominant noise source distribution for a modern vehicle.

Figure 1.1 shows estimated of interior noise levels of the various vehicles' noise sources as they evolved over the last 40 years (Sandberg, 2001). For instance, the exhaust noise have been controlled in succession by single, dual, and triple muffler systems leading to a rate of noise reduction of about $6 \mathrm{~dB}$ per decade. This figure also indicates that in the 1970s and early 1980s reduction of vehicles noise had been concentrated on the power unit. It is also interesting to note that by the late 80's the tire has become the main noise source as the other sources have been significantly attenuated. Except for a tire profile treatment and the use of some compound material, no real technologies have been applied to control tire noise.

As shown by the bibliography research performed by Kuijpers (2001), most of the tire models were mainly developed to predict external radiation. The reason for this fact is the new noise emission limits imposed in several countries including USA, focus on the external radiated noise reduction (Kuijpers, 2001). Several reports on the efforts and difficulties to meet regulations were recently discussed by Rochat (2002) and Sandberg (2001, 2002).

However, the automotive industry continues to strive for interior noise control technologies as part of its effort to improve vehicle noise, vibration, and harshness characteristics. Controlling interior noise due to the tires has, thus, become a topic of great interest to both industry and academia. 
For a modern vehicle, the interior tire noise generation is divided into two mechanisms: the air borne and structure borne paths. The air borne path is the noise generated by the tire that propagates trough the exterior medium (air) and excites the vehicle external shell, resulting in cabin noise as explained by Sandberg and Ejsmont (2002). The main noise source for the air borne path phenomenon is the air displacement induced by the tire tread cavities, i.e. air pumping into and out of the tread cavities. This topic was investigated in depth by Chai et al. (2002) and Klein (2002).

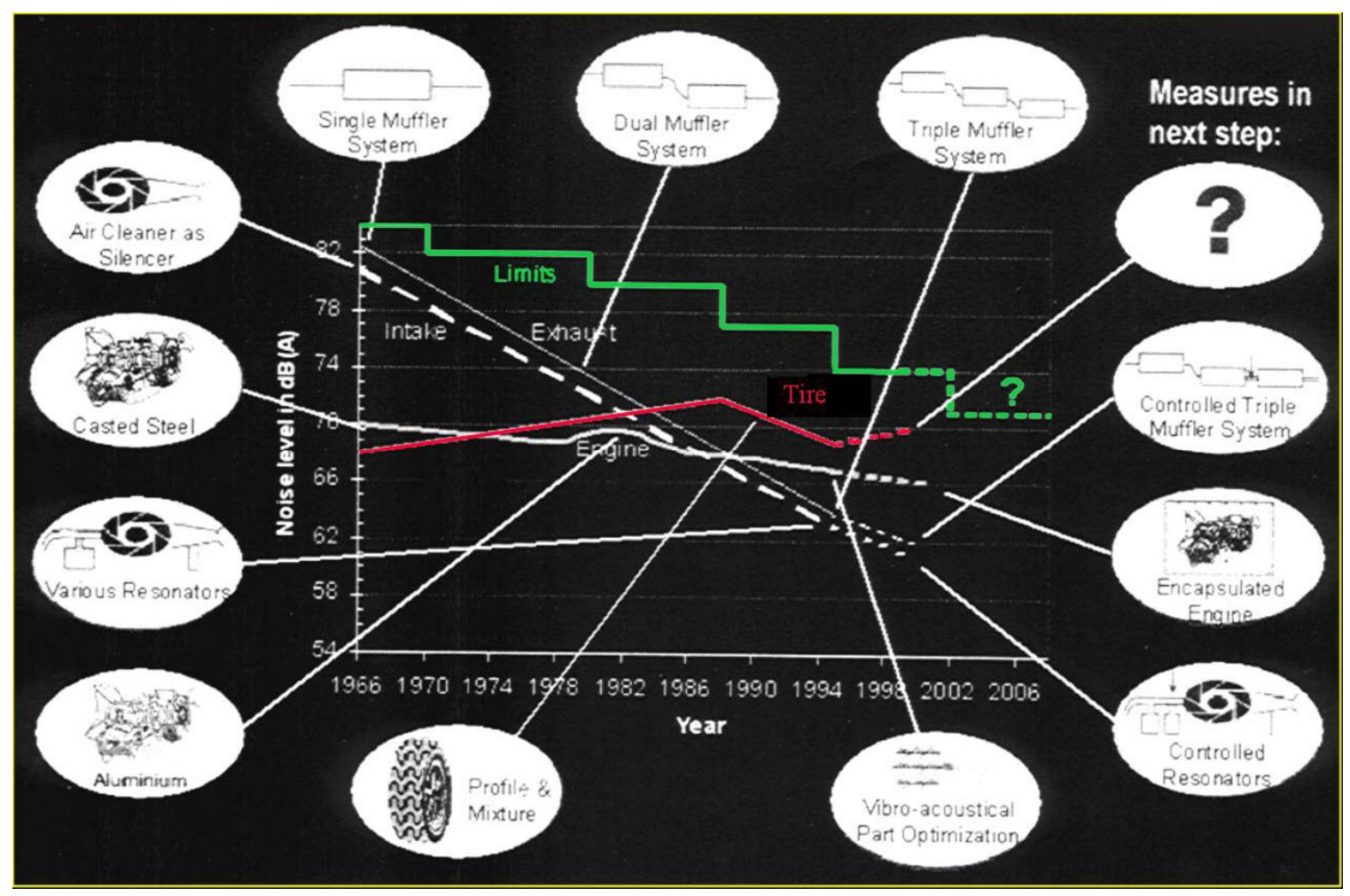

Figure 1.1: Evolution of vehicle noise sources and control technologies (Sandberg, 2001).

On the other hand, the structure borne path is the noise due to the tire vibrations transmitted to vehicle spindle and then to the body structure of the car, resulting in interior noise. The main mechanism responsible for inducing vibrations is the sudden displacement of a tread element, in relation to its "rest position" in the rotating tire when it impacts the road surface. By "rest position" it is meant the position during that part of 
the tire rotation which is not deflected by the road surface contact. The tire is also excited by the adhesion mechanism of the tread and the pavement where stick/slip and stick/snap processes take place. The stick/slip mechanism is due to tangential stresses in the rubberroad interface. This mechanism causes a tangential vibration called "scrubbing”. The stick/snap is due to adhesive bonds between rubber and road which are broken at certain levels when rubber is pulled away from the road contact.

As part of the structural borne mechanism, the tire resonances will greatly influence the forces at spindle and thus the interior noise. From the spindle, the main route of propagation of the vibrations originated by the resonances is believed to be through the solid parts of the vehicle, at least for a frequency range below 400 (Pietrzyk, 2001). Then, the solid parts of the vehicle excite the panels and surfaces of the vehicle cabin, which results in interior noise. It has recently been identified that one such tire resonance that shows rather prominently in the interior noise spectrum is due to the tire acoustic cavity, i.e. resonance of the air column inside the tire cavity. This newly identified effect has created a new challenge for researchers in the vehicle industry and the academic world. The challenge involves the modeling, understanding, and controlling of this tire cavity effect on interior noise. The effect of the acoustic cavity resonance as a new source in the generation of structural born noise is the main concern of this dissertation.

\subsection{LITERATURE REVIEW}

As indicated, the acoustic cavity resonance is a phenomenon recently discovered. The acoustic cavity inside the tire-wheel assembly was revealed to contribute to tire/road noise generation for the first time by Sakata et al. (1990). They performed both vibration and noise measurements in a test vehicle. The vertical point accelerance at the spindle was measured during a coasting test and it is shown in Figure 1.2. Simultaneously, the sound pressure level (SPL) was measured at the driver location. The SPL measurements are shown in Figure 1.3. In the point accelerance, three resonance peaks (indicated as A, 
B, and C) can be seen. From previous studies, the resonance peaks A and C were known to correspond to the tire structural resonances (Sakata et al., 1990). However, the resonance labeled B was not related to any tire structural mode. To further investigate this resonance, the noise results of the coasting test shown in Figure 1.3 were further analyzed. It was noted that the resonance peak B had the same frequency as the peak in the SPL spectrum. It was suspected that peak B in Figure 1.2 was related to the tire acoustic cavity resonance.

An additional road test was performed with the tires filled with polyurethane foam. In this manner, the tire acoustic cavity modes were completely damped out. The sharp peak observed at the frequency of $250 \mathrm{~Hz}$ (peak B) in both the acceleration and noise spectra was eliminated by the foam. From the observation of the experimental results, the resonance at $250 \mathrm{~Hz}$ was attributed to the acoustic cavity resonance. The main implication of this study was that the tire acoustic cavity was an important source on vehicle interior noise that needs to be understood and controlled.

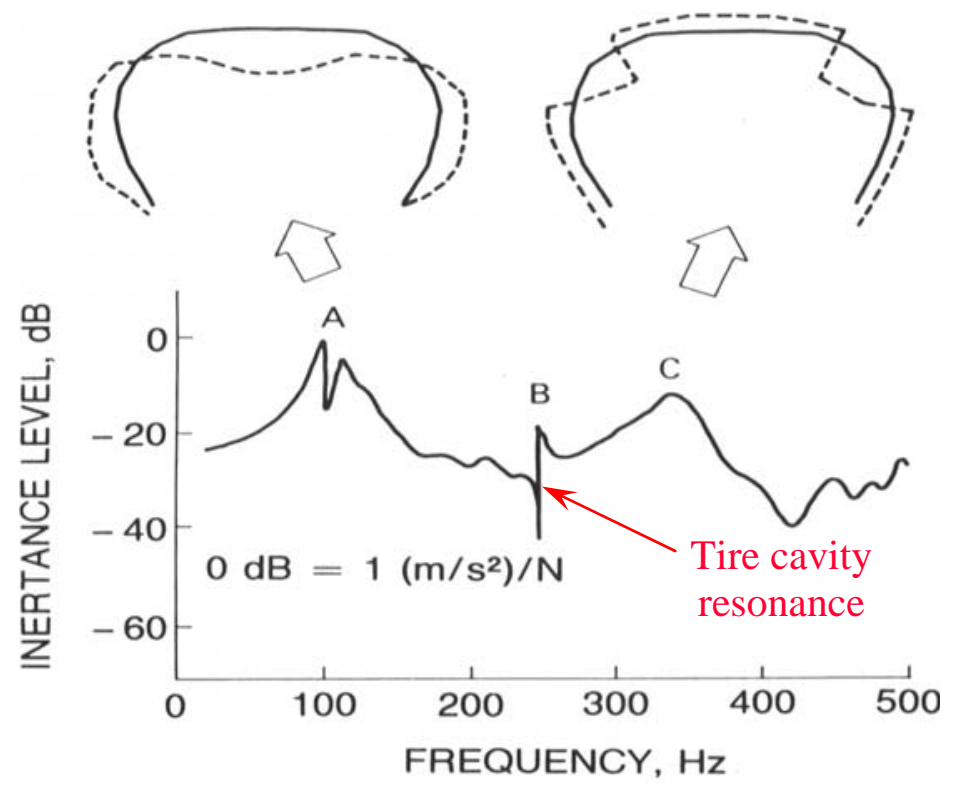

Figure 1.2: Vertical acceleration level at the spindle in a coasting test (Sakata et al. 1990). 
The effect of the tire deformation resulting from the contact with the ground was also investigated in the Sakata et al. (1990) work . Sakata et al. (1990) observed the presence of two acoustic modes for the deformed tire in contact with the ground as compared to the single mode observed in the non-deformed or free tire system. The two modes on the deformed tire occur because of the break in the axis-symmetry due to the contact patch. These modes have the same pattern but one is rotated $90^{\circ}$ relative to the other as shown in Figure 1.4. Therefore, these resonance frequencies were divided into a low and high mode, respectively. The low frequency mode is that for which the contact patch becomes the nodal point, while the high frequency mode is the same mode rotated $90^{\circ}$, i.e. antinode at the contact patch position. The low frequency mode excites the spindle in the horizontal direction, whereas the other mode excites the spindle in the vertical direction. Since the excitation forces are inputs from the road surface, the latter mode had a larger effect in the interior noise (Sakata et al. 1990).

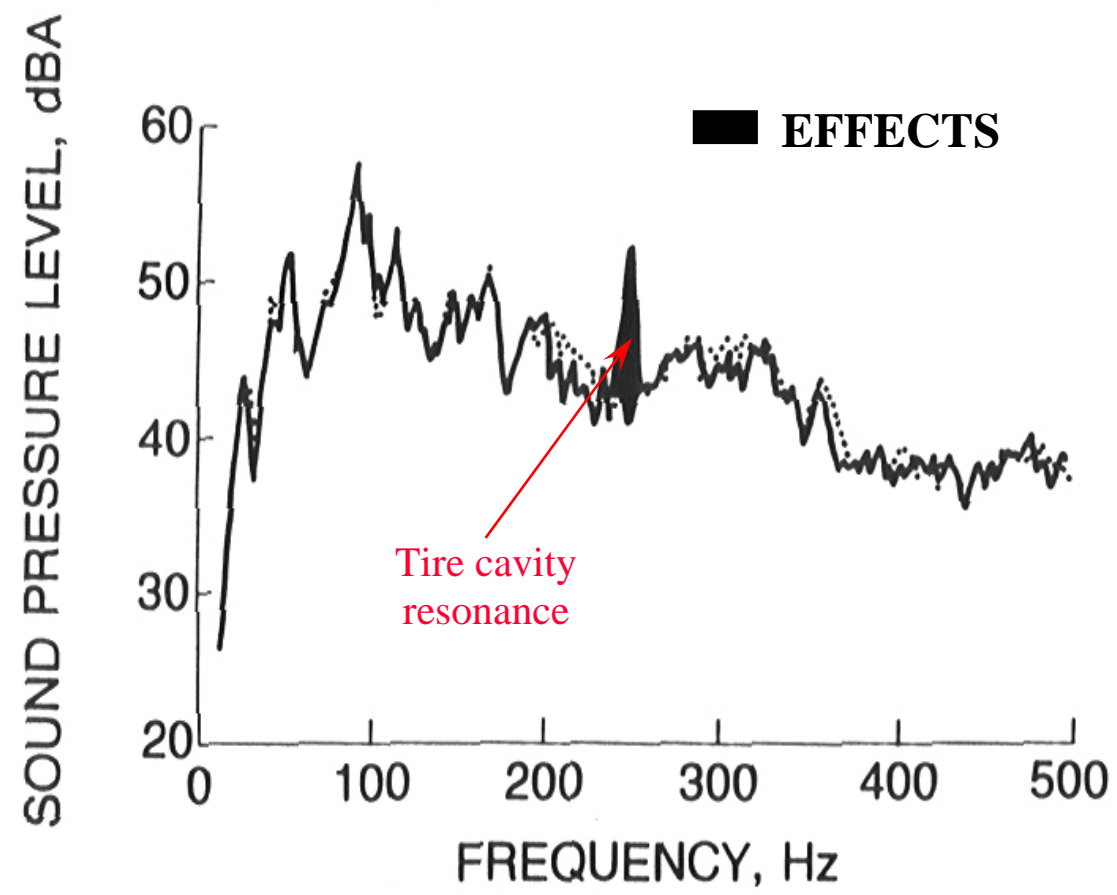

Figure 1.3: Effects of filling the tire cavity with polyurethane foam on the sound pressure level at the driver place in a coasting test (Sakata et al., 1990). 


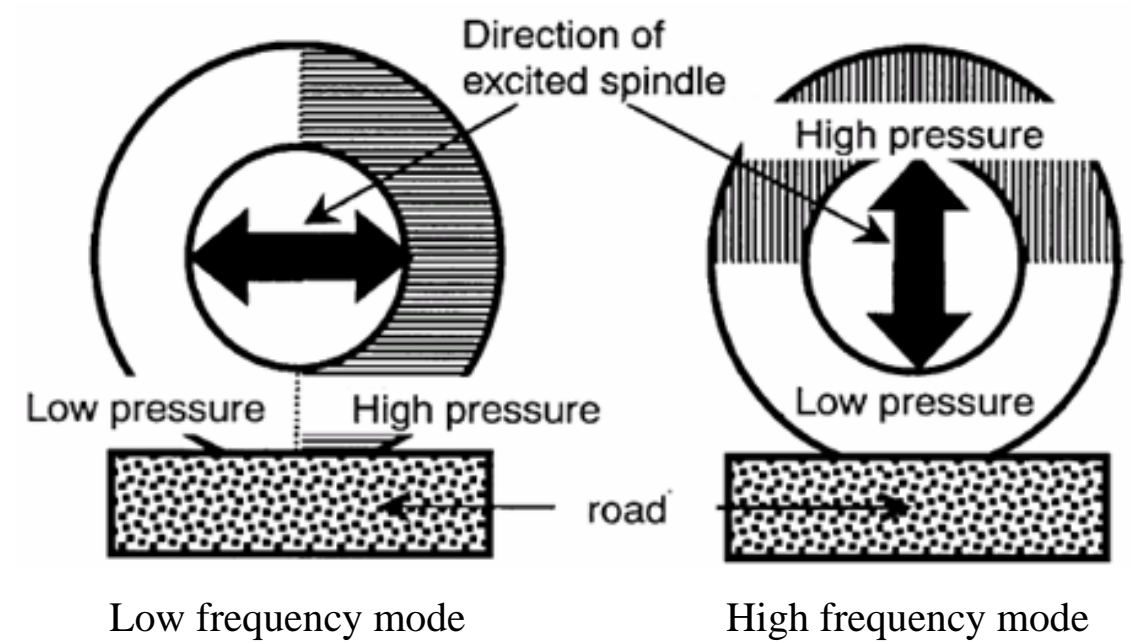

Figure 1.4: Dominating modes of the tire cavity resonance (Yamauchi and Akiyoshi, 2002).

Five years later, Thompson (1995) modeled the tire acoustic cavity by using simple approximate geometrical cavities as shown in Figure 1.5. The models consist of an unwrapped torus representation for the free tire in Figure 1.5a and three connected cavities for the deformed tire as shown in Figure 1.5b. In the case of the deformed tire, the deflected tire acoustic cavity had no longer a constant cross section in the region the tire is in contact with the ground. However, this tire sector was modeled as a constant cross section. In both models, sound in the tire was treated as consisting of plane waves, i.e., low frequency approximation where the wavelength is much greater than the cavity cross sectional dimensions. In addition, the periodicity of the system at the end boundaries was considered. The acoustic cavity natural frequencies were predicted with relatively good accuracy. Thompson (1995) also presented some qualitative information about the direction of the forces at the vehicle spindle. 


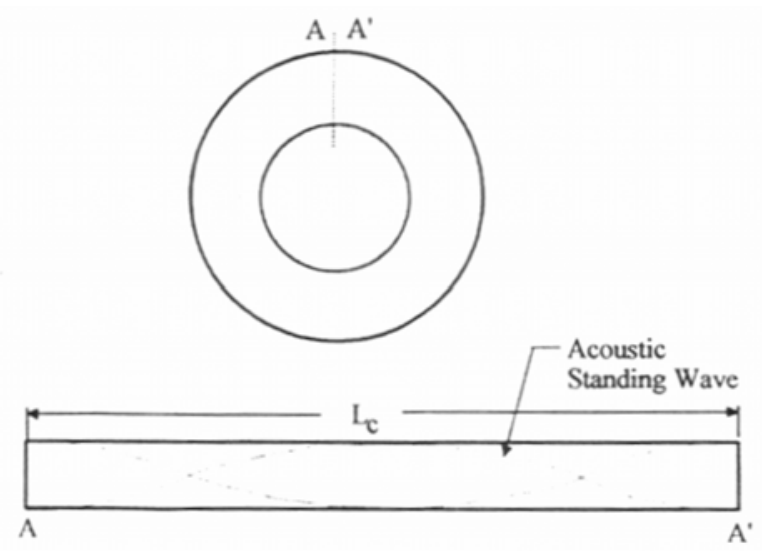

(a)

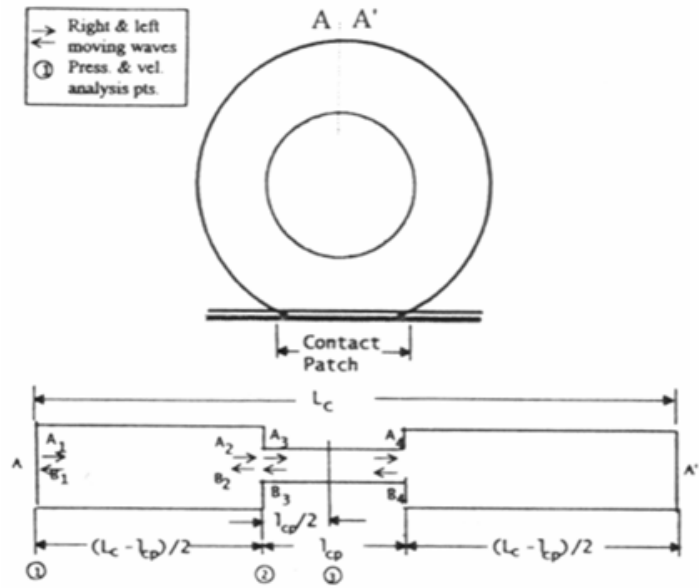

(b)

Figure 1.5: (a) Un-deformed or Free Tire model, (b) Deflected tire model (Thompson, 1995).

For the case of structure born noise, the most effective approach to reduce the noise is by modification of components in the structural path. Thus, it is common practice to develop large detailed computer models of the vehicle components using finite element (FE) models as Nakajima et al. (1992). Subsequently, the component models are integrated using sub-structuring techniques for analysis of the complete system. The tire model is one of such substructures.

Finite element models have been a very common tool used to investigate tire dynamics. Olatunbosun and Burke (2002) presented a complete finite element tire model, which accounts for hub loading, non-linear effects due to the tire inflation, and tire/road contact effects studying the interaction between the tire and the surface of the road. This finite element model did not account for the tire acoustic cavity. Another FE based model accounting for the forces induced on the tire by road irregularities was introduced for Belluzzo et al. (2002). The model was used to study the influence of the different pavement textures upon the tire. This model also excludes the tire acoustic cavity which is going to have a strong influence for some tire size in the range of frequencies that Belluzzo et al. (2002) studied. Darnell and Kestler (2002) presented a finite element model to account for the tire-terrain interaction. The model was used to show the tire 
footprint characteristics such as deflection, areas (contact patch), and stresses for rigid and deformable soil surfaces. Sobhanie (2003) developed a finite element model to study the forces in the tire/suspension system when a quarter car model traverses an obstacle such as pothole or bump. The model presented by this author was developed by using ABAQUS and the tire acoustic cavity was not included in the study.

Clayton and Saint-Cyr (1998) numerically incorporated the dynamics of the tire acoustic cavity into an existing 130,000 degree of freedom tire FE model. These researchers modeled the tire cavity using FE and used three different formulations to take into account the fluid-structure coupling. The results of this approach are shown in Figure 1.6. The Dynamic Stiffness in the vertical direction is shown in this figure. The results from the tire-cavity FE model are shown together with experimental data. The very large peak in the figure corresponds to the tire acoustic cavity resonance. The model underpredicts the tire acoustic cavity resonance. This effect was attributed to modal truncation errors.

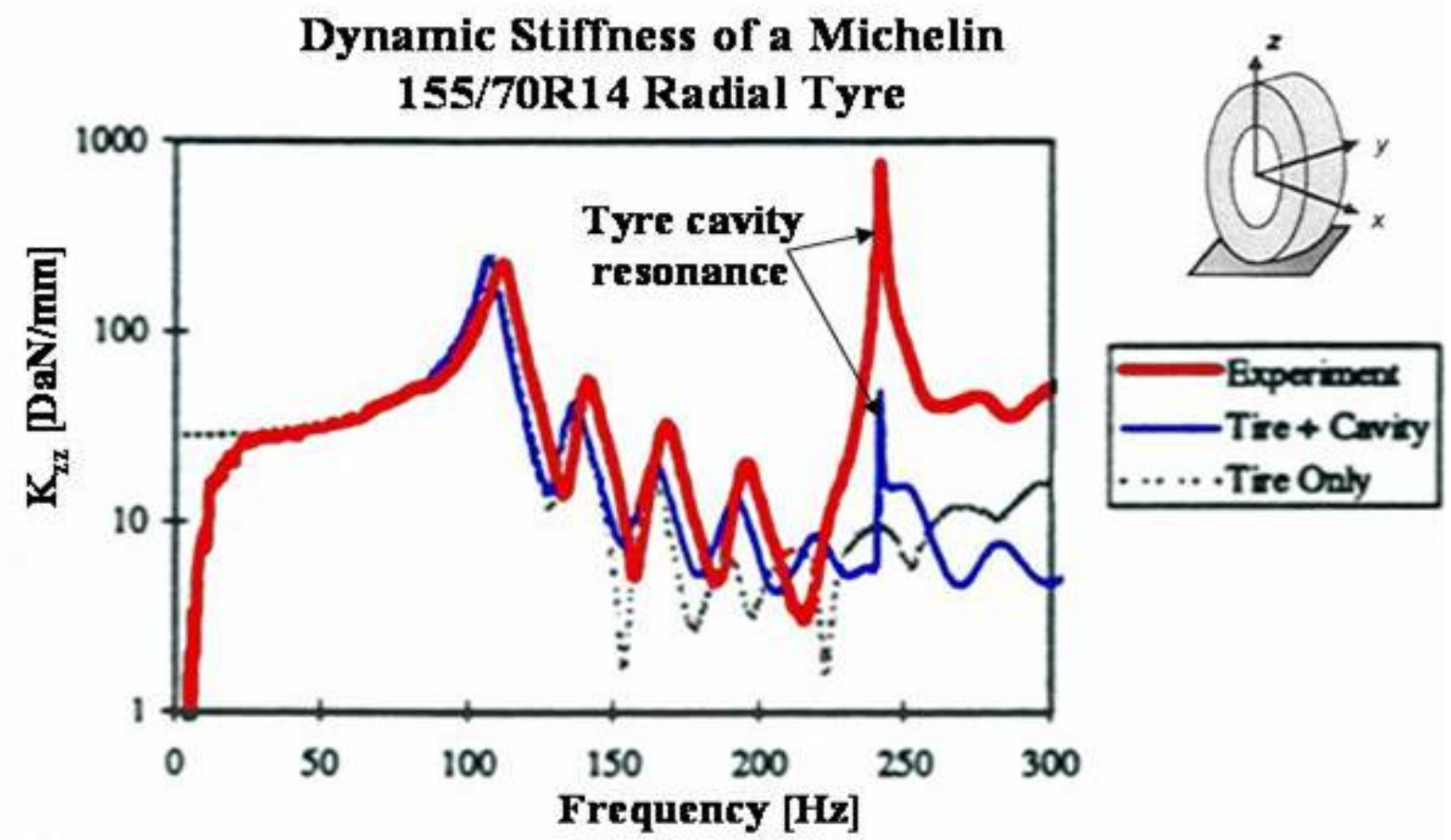

Figure 1.6: Dynamic Stiffness comparison (Clayton and Saint-Cyr, 1998). 
Until 2002, no practical control approach to solve the cavity resonance problem has been presented in the open literature. Yamauchi and Akiyoshi (2002) proposed the first and only open literature control technique to improve the cavity effect in the driving comfort. Yamauchi and Akiyoshi (2002) have used detailed FE models to investigate potential noise control solutions to the cavity acoustic resonance problem. Experiments were also performed to validate the control approaches.

The technique presented to control the tire acoustic cavity resonances by Yamauchi and Akiyoshi (2002) was based in changing the excitation direction. Then, the wheel is no longer exited in the vertical direction and the forces at the spindle are diminished and consequently the interior noise is improved.

It was speculated that in order to accomplish for the change in the direction of excitation, the cavity acoustic system had to be changing with the tire rotation. The changes in the tire acoustic system was achieved by using an oval wheel, i.e., change the tire cross section along the azimuth direction. The oval wheel was created by attaching two identical parts positioned at opposite sides of the rim of the wheel as shown in Figure 1.7. The attached components were configured with closed and open ends. The open ends case results in a secondary cavity inside the attached part. 


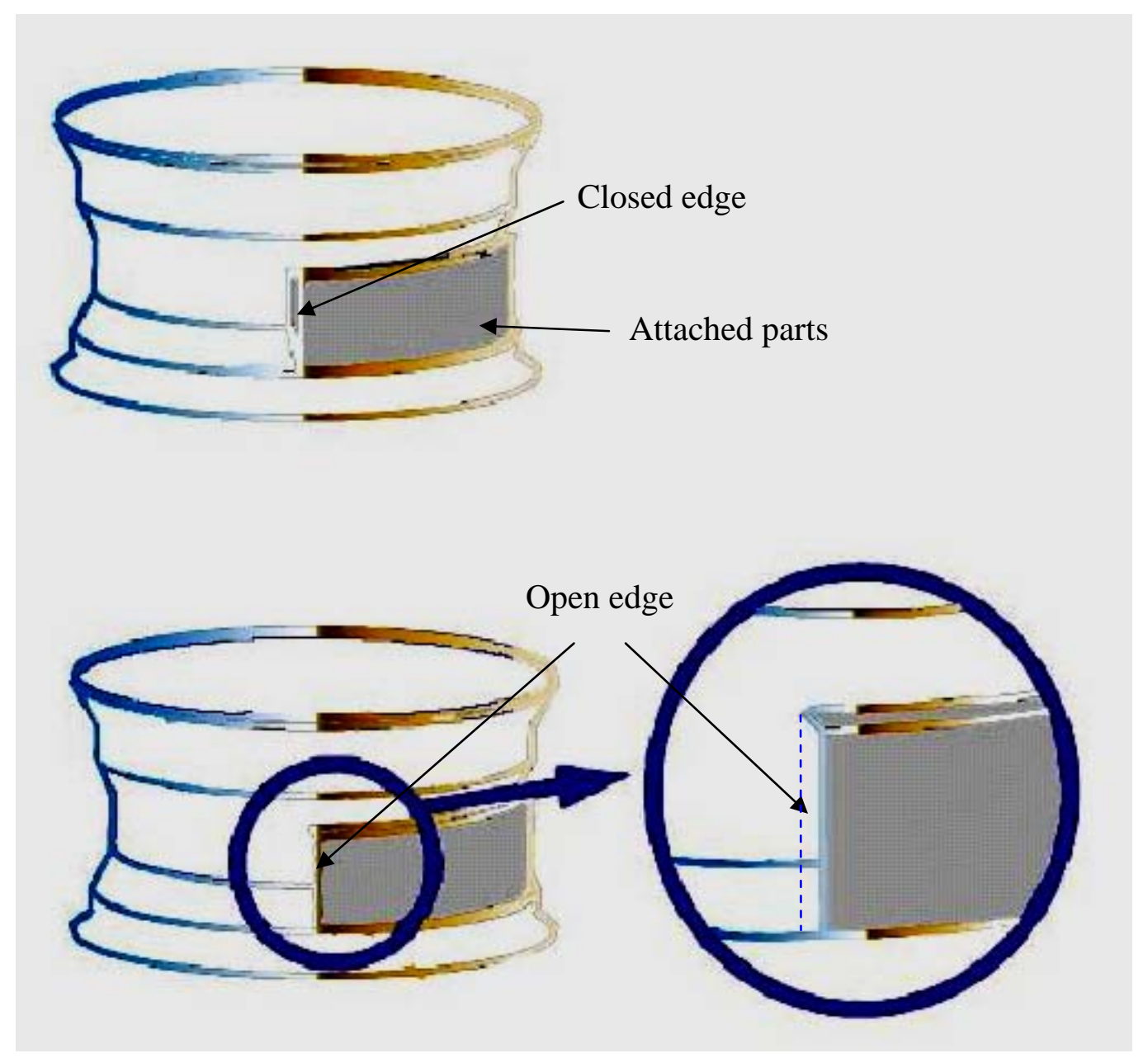

Figure 1.7: trial wheels (Yamauchi and Akiyoshi, 2002)

The control concepts were tested in a standard drum test at $50 \mathrm{~km} / \mathrm{hr}$. The results of the experiment are shown in Figure 1.8. This figure shows the noise level spectrum for the wheel without attachments (in red), the wheel with open edges attachments (in blue), and the wheel with closed edges attachments (in green), respectively. The results show that only "the close edge oval wheel" reduced the cavity resonance by approximately $6 \mathrm{~dB}$. An explanation for the poor performance of the open edge oval wheel case was omitted in this work. 


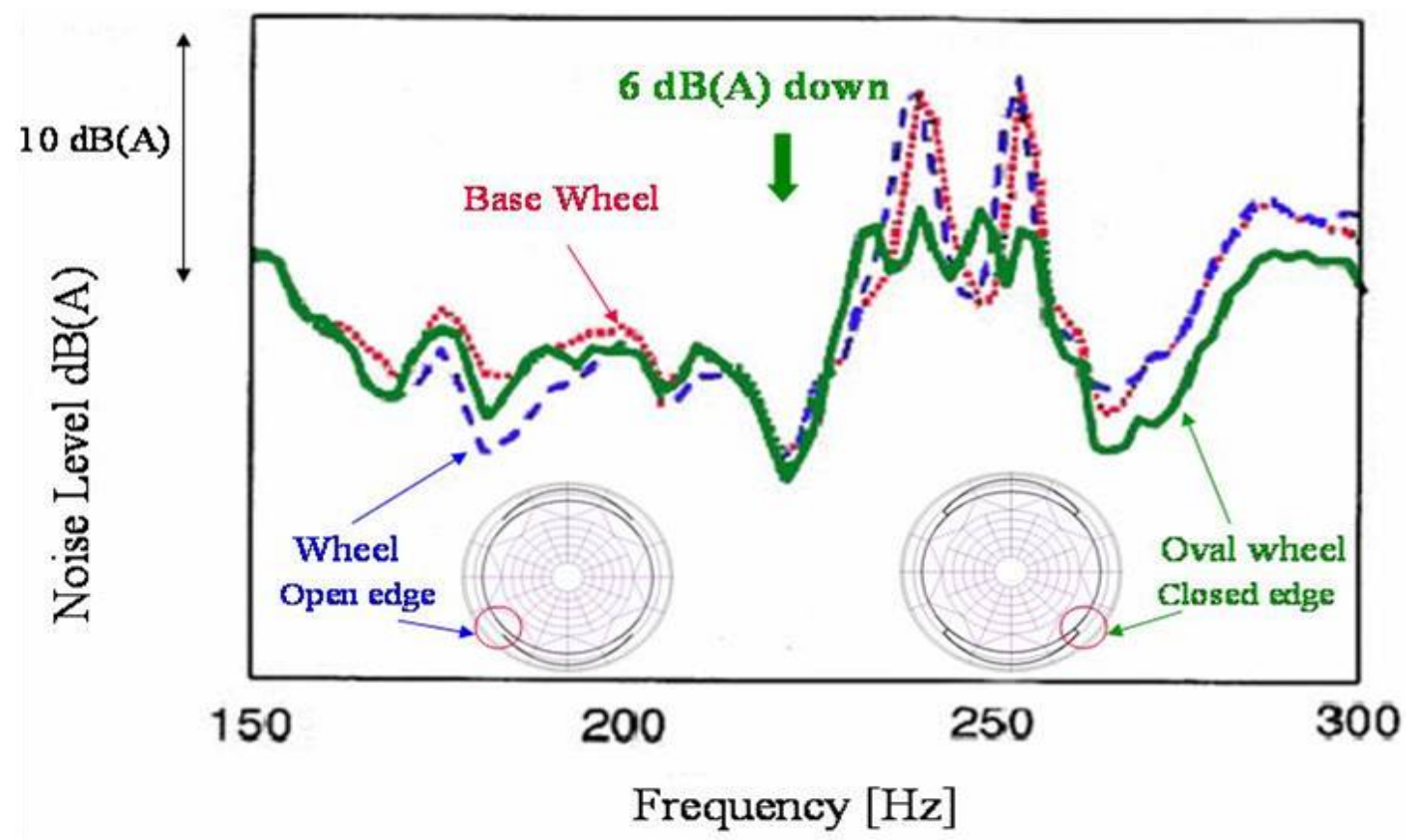

Figure 1.8: Approach to control the cavity effects (Yamauchi and Akiyoshi, 2002).

The literature review reveals limited work related to the problem of interior vehicle noise due to the tire cavity resonance. It can be summarized that: (i) the open literature offers simple models to predict only the natural frequencies of the tire cavity and qualitatively predict the direction of the forces at the vehicle spindle, (ii) most of the modeling effort to compute the tire dynamics have focused on FE techniques that hampers the understanding of the physics involved in the tire-cavity coupling problem, and (iii) the lack of well understood and validated control approaches. Therefore, there is a need for analytical closed form solutions to help gaining insight into the coupling phenomenon and subsequently permit developing practical techniques to control this cavity acoustic resonance problem. 


\subsection{OBJECTIVES OF THIS WORK}

The tire problem is amenable to be modeled analytically leading to close form solutions. The close form solutions can be effectively used to provide valuable insight into the physical mechanisms of the effect that the acoustic cavity resonances have on the transmitted spindle forces. This knowledge can be difficult to uncover from the complexity of numerical solutions such as those provided by Finite Elements or/and Boundary Elements models presented by previous researchers. In addition, the understanding of the physics will help in the design and analysis of potential modifications for the control of this noise source.

The dynamic behavior of the tire acoustic cavity affects the tire response which in turn determines the forces at the spindle. In order to incorporate the dynamics of the cavity, the tire acoustic-structure coupled problem needs to be solved. Moreover, the tire acoustic and structural responses have to be solved simultaneously due to the coupling between them, i.e. fully coupled problem.

Therefore, the main objectives are:

1. To develop an analytical model of the tire to account for the effect of the tire cavity resonances on the spindle forces. A closed form solution model can be developed and be an effective tool, i.e. it can capture in a relative accurate way the physics of the problem.

2. To use the tire model to understand the physics of the mechanism of tire-cavity coupling. In order to identify an effective noise control solution, it is important to understand the physical mechanisms that lead to significant spindle forces due to the tire cavity acoustic resonances.

3. To investigate and model potential techniques to control the cavity effects. After, the coupling mechanism is revealed several control techniques are investigated and 
incorporated into the tire model. Therefore, the model is a valuable tool to qualify interior vehicle noise reduction due to the proposed control approaches.

\subsection{FOREMOST ORIGINAL CONTRIBUTIONS OF THIS WORK}

This dissertation will result in several new contributions to the body of knowledge in this field.

1. Development of the first analytical model of the fully coupled tire structureacoustic cavity. Until now, there are tire models based on very large FE codes or simple models incomplete from the point of view of the quantification of the acoustic cavity effects in the spindle forces. The model developed here is the first ones that model the interaction between the tire structure and acoustic cavity by using close form solutions. The model also incorporates feasible techniques to control the tire acoustic cavity resonances.

2. Allow to gain physical insights into the coupling phenomenon. The versatility of the analytical model permits to investigate the contribution of the acoustic and structural parameters in the coupling phenomenon.

3. Analysis of control techniques to reduce the effect of the tire acoustic cavity resonances. A general modeling approach is developed to investigate potential control techniques that affects the tire cavity resonance using secondary cavities. The technique is also demonstrated using numerical simulations. 


\subsection{DISSERTATION ORGANIZATION}

This dissertation is organized in five chapters with a number of appendixes. Chapter 1 provides an introduction and literature review for this effort. The principal contributions of this work to the current body of knowledge are highlighted.

In Chapter 2, the equations of motion for the tire structure were obtained and the spectral properties computed. The cavity acoustics is not included in this initial formulation. Using the structure eigenproperties, the responses for two tire configurations are developed: (a) response for a "free tire" or un-deformed tire subjected to a point force excitation and (b) the response of the "deformed tire" due to the contact patch induced by the tire/road interaction. In this last case, the excitation is due to the effect of the contact patch due to the roughness of the road. The tire modal responses are then used to compute the forces at the spindle.

Chapter 3 presents the general acoustic problem and the derivation of the acoustic pressure for the interior of the tire. The eigemproblem for the tire acoustic cavity was solved here. This task provided the eigenvalues and eigenfunctions for the cavity sound field. Then, the equations of motion for the coupled system were developed to obtain the modal responses, which were again used to compute the forces at the vehicle spindle.

In Chapter 4, two approaches to control noise are presented. The analytical models developed in chapter 2 and 3 are extended to study these control approaches to suppress the response of the tire cavity acoustic resonances. Here, the first noise control technique uses secondary cavities to control the main tire cavity resonance. The second approach presented is based on adding damping in the interior of the tire main cavity by using viscoelastic screens. 
In Chapter 5, the conclusions and recommendations obtained from the analysis and study of this research project are presented. Several topics are suggested for future research in the tire noise reduction problem.

It is important to emphasize that the results obtained from the analytical models developed in this work were compared against experimental data. This comparison allowed to determine the capability of the models to capture the key features of the acoustic cavity problem. The experimental results were obtained from the work by Yamauchi and Akiyoshi (2002). It was decided to compare appropriate predicted and measured results through out the dissertation as the various models are developed. The predicted versus measured comparison was performed for a tire size 195/65 R15. Appendix A describes the tire size nomenclature. The experimental results are used to validate the tire models presented in Chapters 2 and 3 of this effort. 


\section{CHAPTER 2: STRUCTURAL MODEL}

$\mathrm{T}$

he core of this chapter is the development of the model for the in vacuo tire structure, i.e., without the acoustic cavity included. The modeling of the tire structure includes the formulation for the "un-deformed or free" and “deformed" tire cases. In addition, the formulation to compute the forces at the spindle is presented. The "free tire" is the model of the unsupported tire. In this model, the external excitation is a harmonic point force acting normal to the external surface of the tire. The “deformed tire" development includes the modeling of the contact patch area using a Fourier's ${ }^{1}$ series expansion. A prescribed displacement is imposed on the tire yielding to a static displacement field due to the contact patch. Afterward, a harmonic vertical motion of the tire is superimposed that results in an effective load acting on the tire and the associated dynamic response. A variational method is then used to obtain the resulting forces acting at the spindle, which are transmitted to the vehicle body. Lastly, numerical results for the tire models are shown. Validation of both the "free tire" and the "deformed

\footnotetext{
1 Jean Baptiste Joseph Fourier

Born: March 21, 1768, Auxerre, France.

Died: May 161830 Paris, France.
}

Fourier studied the mathematical theory of heat conduction. He established the partial differential equation governing heat diffusion and solved it by using infinite series of trigonometric functions.

Fourier trained for the priesthood but did not take his vows. Instead took up mathematics studying (1794) and later teaching mathematics at the new École Normale.

In 1798 he joined Napoleon's army in its invasion of Egypt as scientific advisor. He helped establish educational facilities in Egypt and carried out archaeological explorations. He returned to France in 1801 and was appointed prefect of the department of Isere by Napoleon.

He published "Théorie analytique de la chaleur" in 1822 devoted to the mathematical theory of heat conduction. He established the partial differential equation governing heat diffusion and solved it by using infinite series of trigonometric functions. In this he introduced the representation of a function as a series of sines or cosines now known as Fourier series.

Fourier's work provided the impetus for later work on trigonometric series and the theory of functions of real variable.

(Source: http://www.sci.hkbu.edu.hk/scilab/math/fourier.html ) 
tire” model is also included by comparison with experimental results obtained from Yamauchi and Akiyoshi (2002).

\subsection{FREE TIRE MODEL}

For an analytical closed form analysis, the tire was modeled as two shells of revolution and two annular plates as shown in Figure 2.1. In this model, the outer shell was assumed to be the only elastic component while the inner shell and the two annular side plates are considered to be rigid. The outer shell has thickness $h_{\text {Shell }}$, radius $a$, tire width $L_{T}$, and the inner shell radius is $b$. The structure connecting the tire to the spindle is also considered to be rigid. The boundary conditions for the outer shell were assumed to be simply supported, i.e. shear diaphragm edge conditions. The system is also assumed to be stationary, i.e. no rotation.

The equations of motion used for the thin circular cylindrical shell follow the DonnellMushtari theory, (Leissa, 1993, Soedel, 1993, and Ventsel et. al., 2001). The first step in the model of the tire structure is to solve for the eigenvalue problem of the self-adjoint Donnell-Mushtari operator. The displacement vector is defined as $\{u, v, w\}^{\mathrm{T}}$ where $(u)$ is the axial, $(v)$ tangential, and $(w)$ radial components as shown in Figure 2.1. For the simply-supported cylindrical shell of finite length, $L_{T}$, the harmonic displacement vector oscillating a frequency $\omega$ is given as

$$
\left\{u_{i}\right\}=\left\{\begin{array}{l}
u \\
v \\
w
\end{array}\right\}=\left\{\begin{array}{c}
U \cos \left(\frac{m \pi}{L_{T}} x\right) \cos (n \theta+\gamma) \cos (\omega t) \\
V \sin \left(\frac{m \pi}{L_{T}} x\right) \sin (n \theta+\gamma) \cos (\omega t) \\
W \sin \left(\frac{m \pi}{L_{T}} x\right) \cos (n \theta+\gamma) \cos (\omega t)
\end{array}\right\} \begin{gathered}
i=1,2,3 \\
n=0,1,2, \ldots m=1,2,3, . .
\end{gathered}
$$

Note that in this equation the angle $\gamma$ is undefined due to the axis-symmetry of the problem. This angle is determined by the position of the external load. For the sake of 
clarity, the angle $\gamma$ is set to zero for the rest of the derivation since the external load will be acting along the vertical direction.

In equation (2.1), each pair of indices $(m, n)$ define the modal pattern, i.e. $m$ and $n$ define the axial and azimuth variation of the mode shape, respectively. The displacement vector in (2.1) defines the mode shapes.

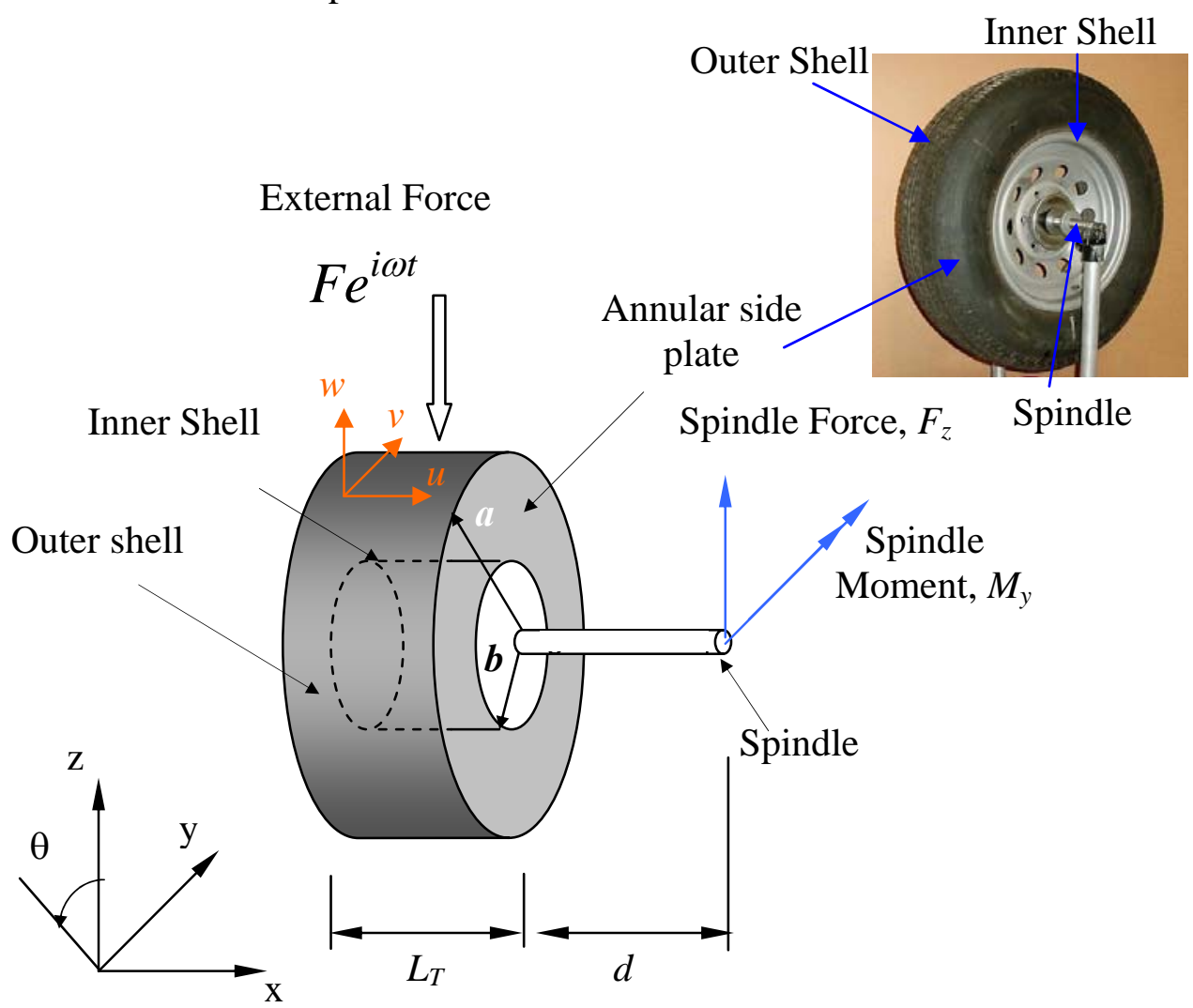

Figure 2.1: Simplified tire model.

To find the natural frequencies, the Donnel-Mushtari-Vlasov operator is applied on the displacement vector as

$$
\left[\begin{array}{ccc}
a^{2} \frac{\partial^{2}}{\partial x^{2}}+\frac{\left(1-v^{2}\right)}{2} \frac{\partial^{2}}{\partial \theta^{2}}-\rho \frac{\left(1-v^{2}\right) a^{2}}{E} \frac{\partial^{2}}{\partial t^{2}} & \frac{(1+v) a}{2} \frac{\partial^{2}}{\partial x \partial \theta} & v a \frac{\partial}{\partial x} \\
\frac{(1+v) a}{2} \frac{\partial^{2}}{\partial x \partial \theta} & \frac{\partial^{2}}{\partial \theta^{2}}+\frac{\left(1-v^{2}\right) a^{2}}{2} \frac{\partial^{2}}{\partial x^{2}}-\rho \frac{\left(1-v^{2}\right) a^{2}}{E} \frac{\partial^{2}}{\partial t^{2}} & \frac{\partial}{\partial \theta} \\
v a \frac{\partial}{\partial x} & \frac{\partial}{\partial \theta} & 1+K \nabla^{4}+\rho \frac{\left(1-v^{2}\right) a^{2}}{E} \frac{\partial^{2}}{\partial t^{2}}
\end{array}\right] \cdot\left\{\begin{array}{c}
u \\
v \\
w
\end{array}\right]=\left\{\begin{array}{l}
0 \\
0 \\
0
\end{array}\right]\left(\begin{array}{l}
2 \\
2.2
\end{array}\right.
$$


where $\rho$ is the tire material density, $E$ is the tire Young's Modulus, $v$ is the Poison ratio, and $K$ is the nondimensional tire thickness parameter given by

$$
K=\frac{h_{\text {Shell }}^{2}}{12 a^{2}}
$$

Replacing (2.1) into (2.2) leads to

$$
\left[\begin{array}{ccc}
-\lambda_{m}^{2}-\frac{\left(1-v^{2}\right)}{2} n^{2}+\Omega^{2} & \frac{(1+v)}{2} n \lambda_{m} & v \lambda_{\mathrm{m}} \\
\frac{(1+v)}{2} n \lambda_{m} & (1-v) \lambda_{m}^{2}-n^{2}+\Omega^{2} & -n \\
-v \lambda_{m} & n & 1+K\left(\lambda_{m}^{2}+n^{2}\right)^{2}-\Omega^{2}
\end{array}\right] \cdot\left\{\begin{array}{l}
U \\
V \\
W
\end{array}\right\}=\left\{\begin{array}{l}
0 \\
0 \\
0
\end{array}\right\}
$$

where $\Omega$ is referred as the normalized frequency, and is defined as

$$
\Omega=\sqrt{\frac{\rho\left(1-v^{2}\right)}{E}} a \omega
$$

and

$$
\lambda_{m}=\frac{m \pi a}{L_{T}} \quad \text { with } \quad m=1,2,3, \ldots \lim _{\delta x \rightarrow 0}
$$

The eigenvalue problem in (2.4) will yield the shell natural frequencies and mode shapes. For each pair $(m, n)$ defining a response pattern, the eigenvalue problem results in three 
natural frequencies which define three modes. In general, these modes are characterized by the dominance of one of the displacement vector component, i.e. longitudinally (i.e. $U_{m n}^{(j)}>W_{m n}^{(j)}$ and $U_{m n}^{(j)}>V_{m n}^{(j)}$ ), tangentially (i.e. $V_{m n}^{(j)}>U_{m n}^{(j)}$ and $V_{m n}^{(j)}>W_{m n}^{(j)}$ ), and radially (i.e. $W_{m n}^{(j)}>U_{m n}^{(j)}$ and $\left.W_{m n}^{(j)}>V_{m n}^{(j)}\right)$ dominated modes. The eigenvalues and eigenvectors are:

$$
\Omega_{m n}^{(j)} \quad \text { and } \quad\left\{\begin{array}{l}
U_{m n}^{(j)} \\
V_{m n}^{(j)} \\
W_{m n}^{(j)}
\end{array}\right\} \quad \text { for }(m, n) \quad j=1,2,3
$$

By replacing the eigenvector given in equation (2.7) into (2.1) leads to the eigenfunctions (or mode shapes)

$$
\left\{\Phi_{m n}^{(j)}\right\}=\left\{\begin{array}{l}
U_{m n}^{(j)} \cos (n \theta) \cos \left(\lambda_{m} \frac{x}{a}\right) \\
V_{m n}^{(j)} \sin (n \theta) \sin \left(\lambda_{m} \frac{x}{a}\right) \\
W_{m n}^{(j)} \cos (n \theta) \sin \left(\lambda_{m} \frac{x}{a}\right)
\end{array}\right\}
$$

Characteristic radial modal patterns for a circular cylindrical shell supported at both ends by "shear diaphragms" are shown in Figure 2.2. For instance, the $\Phi_{11}^{(1)}$ structural mode represents a half sine wave in the longitudinal direction and a complete cosine wave in the azimuth direction. The mode shapes with azimuthal index $n=0$ are referred to as "breathing” modes, while for $n=1$, they are noted as "bending” modes because the shell behaves similarly to a beam, i.e. no cross section deformation. 


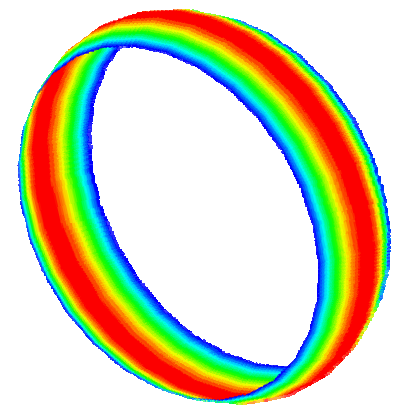

Structural Mode $\Phi_{10}^{(1)}$

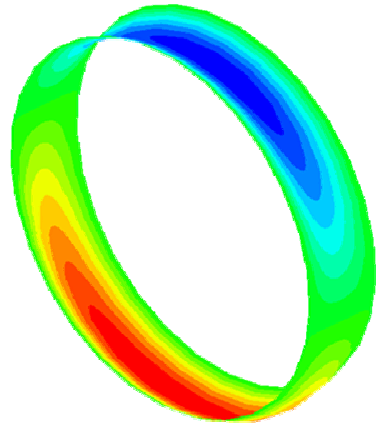

Structural Mode $\Phi_{11}^{(1)}$

Figure 2.2: Radial nodal patters for circular cylindrical shells.

For the forced response without considering the tire acoustic cavity coupling, the differential equation of motion is now given by

$$
\left(\left[L_{c}\right]-\Omega^{2}[I]\right)\left\{\begin{array}{l}
u \\
v \\
w
\end{array}\right\}=\left\{\begin{array}{l}
f_{u} \\
f_{v} \\
f_{w}
\end{array}\right\}
$$

where $\Omega$ is the normalized excitation frequency defined in equation (2.5). Here, the external load vector $\left\{f_{u}, f_{v}, f_{w}\right\}^{T}$ is assumed to be due to a harmonic point force as shown in Figure 2.1. Note that $f_{u}, f_{v}$, and $f_{w}$ are the axial, azimuthal, and radial components of the external load, respectively. The force amplitude is $F$, and $x_{f}$ and $\theta_{o}$ are the position in the axial and azimuth direction, respectively. The external force vector is then given by

$$
f_{u}=f_{v}=0 \text { and } f_{w}=F \cdot \delta\left(\theta-\theta_{o}\right) \cdot \delta\left(x-x_{f}\right) \cdot e^{i \omega t}
$$


where $\delta($.$) is the Dirac's delta function and \left[L_{c}\right]$ is the elastic component of the shell operator defined in (2.4) given as

$$
\left[L_{c}\right]=\left[\begin{array}{ccc}
a^{2} \frac{\partial^{2}}{\partial x^{2}}+\frac{\left(1-v^{2}\right)}{2} \frac{\partial^{2}}{\partial \theta^{2}} & \frac{(1+v) a}{2} \frac{\partial^{2}}{\partial x \partial \theta} & v a \frac{\partial}{\partial x} \\
\frac{(1+v) a}{2} \frac{\partial^{2}}{\partial x \partial \theta} & \frac{\partial^{2}}{\partial \theta^{2}}+\frac{\left(1-v^{2}\right) a^{2}}{2} \frac{\partial^{2}}{\partial x^{2}} & \frac{\partial}{\partial \theta} \\
v a \frac{\partial}{\partial x} & \frac{\partial}{\partial \theta} & 1+K \nabla^{4}
\end{array}\right]
$$

The response is now expressed in terms of a linear combination of the modes as

$$
\left\{\begin{array}{l}
u \\
v \\
w
\end{array}\right\}=\sum_{m=1}^{M} \sum_{n=0}^{N}\left[\Phi_{m n}\right]\left\{\begin{array}{c}
A_{m n}^{(1)} \\
A_{m n}^{(2)} \\
A_{m n}^{(3)}
\end{array}\right\} e^{i \omega t}
$$

where $A_{m n}^{(j)}$ are the modal amplitudes of the $(m, n)$ mode for the $j^{\text {th }}$ component and $\left[\Phi_{m n}\right]$ is the 3x3 $(m, n)$ modal matrix, i.e. $\left[\Phi_{m n}\right]=\left[\left\{\Phi_{m n}^{(1)}\right\}\left\{\Phi_{m n}^{(2)}\right\}\left\{\Phi_{m n}^{(3)}\right\}\right]$.

To solve for the modal amplitudes, equation (2.12) is replaced in (2.9) pre-multiplied by the transpose of the modal matrix, and integrated over the surface of the cylinder. That is:

$$
\int_{0}^{L} \int_{0}^{2 \pi} \sum_{m=1}^{M} \sum_{n=0}^{N}\left[\Phi_{r s}\right]^{T}\left(\left[L_{c}\right]-\Omega^{2}[I]\right) \sum_{m=1}^{M} \sum_{n=0}^{N}\left[\Phi_{m n}\right]\left\{\begin{array}{l}
A_{m n}^{(1)} \\
A_{m n}^{(2)} \\
A_{m n}^{(3)}
\end{array}\right\} a d x d \theta=\int_{0}^{L} \int_{0}^{2 \pi}\left[\Phi_{r s}\right]^{T}\left\{\begin{array}{l}
f_{u} \\
f_{v} \\
f_{w}
\end{array}\right\} a d x d \theta
$$

Expanding equation (2.13) as follow: 


$$
\sum_{m=1}^{M} \sum_{n=0}^{N}\left[\int_{0}^{L 2 \pi} \int_{0}^{2 \pi}\left[\Phi_{r s}\right]^{T}\left[L_{c}\right]\left[\Phi_{m n}\right] a d \theta d x-\Omega^{2} \int_{0}^{L 2 \pi} \int_{0}^{2 \pi}\left[\Phi_{r s}\right]^{T}[I]\left[\Phi_{m n}\right] a d \theta d x\right] \cdot\left\{\begin{array}{l}
A_{m n}^{(1)} \\
A_{m n}^{(2)} \\
A_{m n}^{(3)}
\end{array}\right\}=\int_{0}^{L 2 \pi} \int_{0}^{2 \pi}\left[\Phi_{r s}\right]^{T}\left\{\begin{array}{l}
f_{u} \\
f_{v} \\
f_{w}
\end{array}\right\} a d \theta d x
$$

Considering the orthogonality properties of the mode shapes leads to the modal mass $\left[M_{m n}\right]$ and modal stiffness $\left[K_{m n}\right]$ matrices as follow

$$
\int_{0}^{L} \int_{0}^{2 \pi}\left[\Phi_{m n}\right]^{T}[I]\left[\Phi_{m n}\right] a d \theta d x=\left[M_{m n}\right]=\left[\Phi_{m n}\right]^{T}\left[\Phi_{m n}\right] \varepsilon_{n} \frac{\pi}{2} a L=\left[\hat{M}_{m n}\right] \varepsilon_{n} \frac{\pi a L}{2}
$$

and

$$
\int_{0}^{L} \int_{0}^{2 \pi}\left[\Phi_{r s}\right]^{T}\left[L_{c}\right]\left[\Phi_{m n}\right] a d \theta d x=\left[K_{m n}\right]=\left[M_{m n}\right] \cdot\left\{\Omega_{m n}^{2}\right\}
$$

where

$$
\varepsilon_{n}=\left\{\begin{array}{l}
2 \text { for } \mathrm{n}=0 \\
1 \text { for } \mathrm{n}>0
\end{array}\right.
$$

The right hand side of (2.14) results in the generalized force vector

$$
\left\{f_{m n}\right\}=\left\{\begin{array}{l}
f_{m n}^{(1)} \\
f_{m n}^{(2)} \\
f_{m n}^{(3)}
\end{array}\right\}=\int_{0}^{L} \int_{0}^{2 \pi}\left[\Phi_{r s}\right]^{T}\left\{\begin{array}{l}
f_{u} \\
f_{v} \\
f_{w}
\end{array}\right\} a d \theta d x=a\left\{\begin{array}{l}
W_{m n}^{(1)} \\
W_{m n}^{(2)} \\
W_{m n}^{(3)}
\end{array}\right\} \sin \left(\lambda_{m} \frac{x_{f}}{a}\right) \cos \left(n \theta_{o}\right)
$$

Thus, the modal amplitudes are obtained by solving the following uncoupled system of algebraic equations

$$
\left[\left[K_{m n}\right]-\Omega^{2}\left[M_{m n}\right]\right]\left\{\begin{array}{l}
A_{m n}^{(1)} \\
A_{m n}^{(2)} \\
A_{m n}^{(3)}
\end{array}\right\}=\left\{f_{m n}\right\} \quad \begin{gathered}
n=0,1,2, \ldots, N \\
m=1,2,3, \ldots, M
\end{gathered}
$$




$$
\left[\begin{array}{ccc}
K_{m n}^{(1)}-\Omega^{2} M_{m n}^{(1)} & 0 & 0 \\
0 & K_{m n}^{(2)}-\Omega^{2} M_{m n}^{(2)} & 0 \\
0 & 0 & K_{m n}^{(3)}-\Omega^{2} M_{m n}^{(3)}
\end{array}\right] \cdot\left\{\begin{array}{l}
A_{m n}^{(1)} \\
A_{m n}^{(2)} \\
A_{m n}^{(3)}
\end{array}\right\}=\left\{\begin{array}{l}
f_{m n}^{(1)} \\
f_{m n}^{(2)} \\
f_{m n}^{(3)}
\end{array}\right\}
$$

The modal amplitudes obtained in the uncoupled system of equation (2.19) are replaced back into equation (2.12) to obtain the displacement field. The damping in the tire structure is incorporated in the equation of motion by using the complex Young's modulus as

$$
E_{c}=E\left(1+i \eta_{\text {Shell }}\right)
$$

where $\eta_{\text {Shell }}$ is the structural loss factor and $i$ is the imaginary unit, i.e. $i=\sqrt{-1}$.

\subsection{DEFORMED TIRE MODEL}

The aim of this section is to derive the model of the dynamic response of a deformed tire in vertical harmonic motion. The general steps in the development of the deformed tire model are shown in Figure 2.3. To this end, the deformation of the tire in the region in contact with the ground needs to be defined, i.e. contact patch model. In this work, the geometry of the contact patch area (footprint) follows the modeling approach used by Darnell and Kestler (2002) and Sobhanie (2003). The region of the tire deformed due to the contact with the ground is assumed to be perfectly defined by the geometry of the problem. That is, the tire deforms from a circular sector of curvature $1 / a$ to a flat surface as shown in Figure 2.3a. The contact patch imposes a prescribed deformation of the tire that needs to be defined. The size of this region (footprint) is completely defined by the static contact patch depth, $\bar{h}$. The prescribed deformation function in turn induces a static displacement field on the rest of the tire which is shown in Figure 2.3b. This static 
displacement field is obtained by using the classical equations for the elastic shells. After the static displacement field is obtained, a harmonic motion of the contact patch $h(t)$ around the equilibrium position defined by the contact patch depth $\bar{h}$ is superimposed, i.e. $h(t)=\bar{h}+\varepsilon e^{i \omega t}$ with $\varepsilon \ll \bar{h}$. This contact patch harmonic motion produces a dynamic displacement field due to the inertia properties of the elastic shell. A representation of the dynamic displacement is shown in Figure 2.3c. Thus, the formulation of the deformed tire case involves the modeling of the prescribed, static, and dynamic displacement fields. They are described in the following sections.

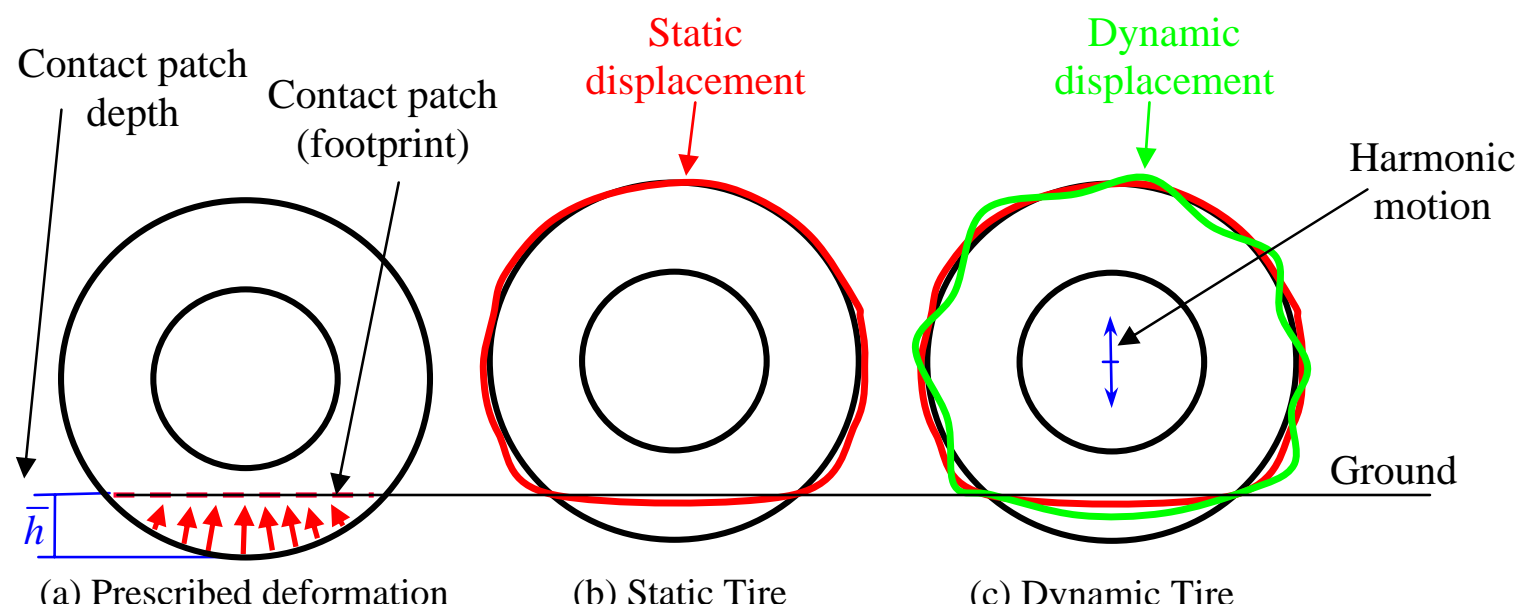

Figure 2.3: Schematic of the contact patch modeling.

Prescribed Deformation: The prescribed displacement is assumed symmetric with respect to $\theta=\pi$. The prescribed displacement function $d(x, \theta)$ is assumed to be affecting only the radial direction, i.e. a point on the tire in contact with the ground moves along the radial direction. This modeling assumption is critical for the next step in finding the static displacement. 


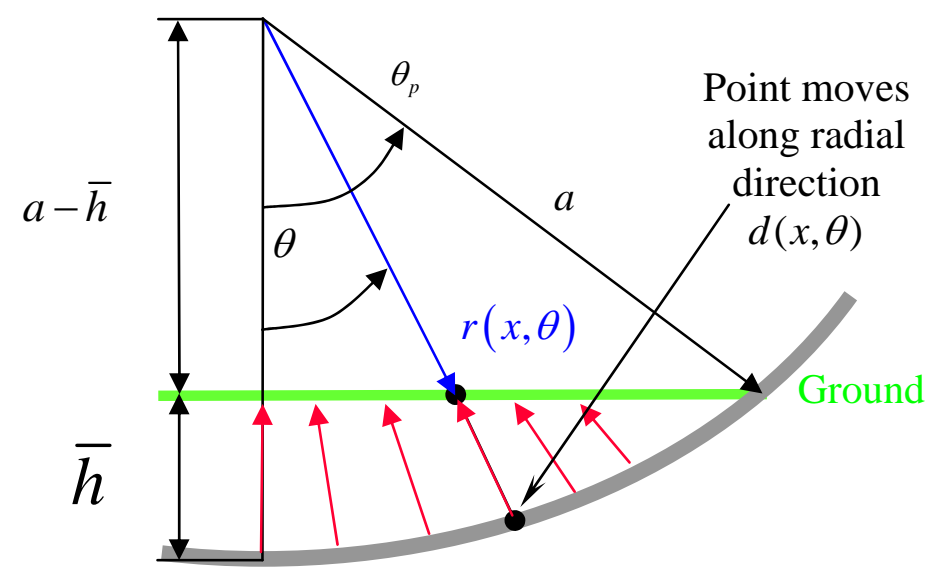

Figure 2.4: Contact patch definition at $x=L_{T} / 2$.

The domain of definition for the "contact patch" is given by

$$
D=\left\{x / x \in\left[0, L_{T}\right] \wedge \theta / \theta \in\left[\theta_{p}(\bar{h}),-\theta_{p}(\bar{h})\right]\right\}
$$

The following geometrical relations are obtained from Figure 2.4,

$$
\cos (\pi-\theta)=\frac{a-\bar{h}}{r(x, \theta)}
$$

and

$$
\cos \left(\pi-\theta_{p}(\bar{h})\right)=\frac{a-\bar{h}}{a}
$$

An explicit functional relationship between $\theta_{p}$ and $h(t)$ can be obtained from expression (2.23) as

$$
\theta_{p}=\pi-\cos ^{-1}\left[\frac{a-\bar{h}}{a}\right]
$$

Note that this is a non linear relation between $\theta_{p}$ and $\bar{h}$. 
Solving for $a-\bar{h}$ in equation (2.22) and substituting into equation (2.23), the value of $r(x, \theta)$ yields

$$
r(x, \theta)=a \frac{\cos \left(\pi-\theta_{p}(\bar{h})\right)}{\cos (\pi-\theta)}
$$

The value of the prescribed displacement is then given by

$$
d(x, \theta)=a\left[\frac{a-\bar{h}}{a \cos (\pi-\theta)}-1\right]\left[H\left(x-L_{T}\right)-H(x-0)\right]
$$

or

$$
d(x, \theta)=\{[\bar{h}-a] \sec (\theta)-a\}\left[H\left(x-L_{T}\right)-H(x-0)\right]
$$

where $H($.$) is the Heaviside's unit step function.$

The prescribed deformation function given by equation (2.27) is illustrated in Figure 2.5 assuming $\bar{h}=0.01 \mathrm{~m}$ for a tire size 195/65 R15. 


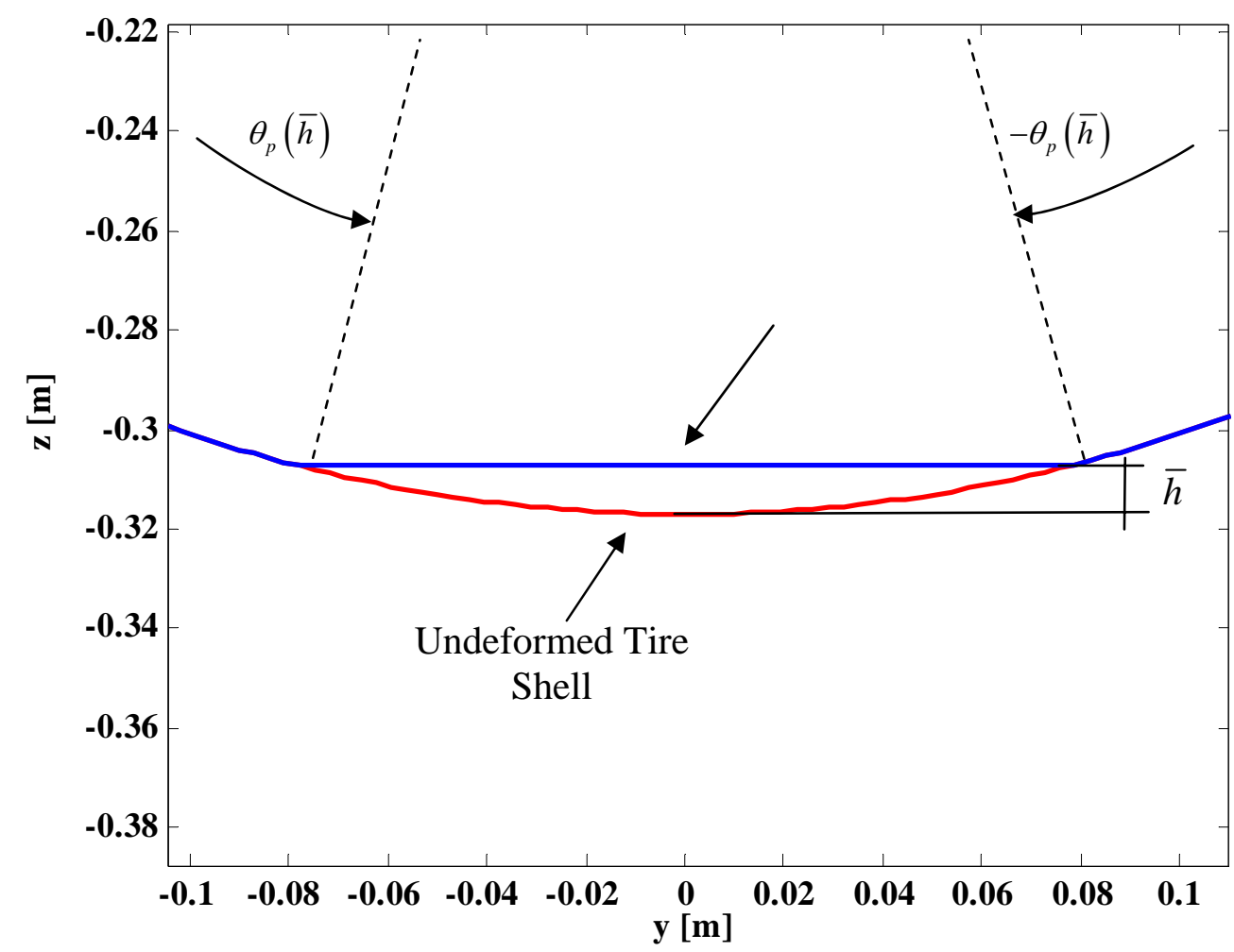

Figure 2.5: Prescribed deformation function $d\left(L_{T} / 2, \theta\right)$ for a tire size 195/65 R15 and

$$
\bar{h}=0.01 m \text {. }
$$

Static Displacement: The prescribed deformation function $d(x, \theta)$ induces a static deformation in the elastic shell as shown in Figure 2.3b. In order to find the static displacement, the prescribed deformation is approximated by the eigenfunctions of the shell. The selection of these functions simplifies the approach because they satisfy the shell equations and the simply supported boundary conditions ("shear diaphragms”). That is,

$$
\begin{aligned}
& w^{S}(x, \theta)=\sum_{m}^{M} \sum_{n}^{N} W_{m n}^{(3)} \sin \left(\frac{n \pi}{L_{T}} x\right) \cos (n \theta) \quad \text { with } \quad m=1,2,3, \ldots, M \\
& n=0,1,2, \ldots, N
\end{aligned}
$$

Note that the static displacement in (2.28) is written only for the radial component of the displacement field. The other two components of the displacement field will be defined 
later. The aim now is to solve for the unknown amplitude $W_{m n}^{(3)}$. To this end, the radial component of the static displacement is forced to match the prescribed displacement (defined only in term of the radial displacement component) over the contact patch sector. It is important to remark that this "matching" can be carried out because the prescribed displacement was defined as a radial displacement. That is

$$
d(x, \theta)=w^{s}(x, \theta) \quad \text { on } \quad 0<x<L, \quad-\theta_{\mathrm{p}}<\theta<\theta_{\mathrm{p}}
$$

Replacing equation (2.28) into equation (2.29), and integrating over the domain defined by the contact patch, it gives

$$
\begin{aligned}
& \int_{O}^{L} \int_{-\theta_{\mathrm{p}}}^{\theta_{\mathrm{p}}} d(x, \theta) \sin \left(s \frac{\pi}{L_{T}} x\right) \cos (r \theta) a d \theta d x= \\
& =\int_{O}^{L} \int_{-\theta_{\mathrm{p}}}^{\theta_{\mathrm{p}}}\left[\sum_{m}^{M} \sum_{n}^{N} W_{m n}^{(3)} \sin \left(\frac{n \pi}{L_{T}} x\right) \cos (n \theta) \sin \left(\frac{s \pi}{L_{T}} x\right) \cos (r \theta)\right] a d \theta d x
\end{aligned}
$$

that represents a linear system of equations as

$$
\left[I_{n r}(\bar{h})\right]\left\{W_{s n}^{(3)}\right\}=\left\{f_{s r}(\bar{h})\right\}
$$

where the matrices $\left[I_{n r}(\bar{h})\right]$ and $\left\{f_{s r}(\bar{h})\right\}$ are fully populated and can be found in Appendix B. The vector $\left\{W_{s n}^{(3)}\right\}$ is obtained by inverting the matrix $\left[I_{n r}(\bar{h})\right]$.

To illustrate the prescribed and static displacement fields several results are shown in Figures 2.6 to 2.8. Figure 2.6 shows the radial displacement of the shell center line for the static problem induced by the prescribed deformation function $d(x, \theta)$ for $\bar{h}=0.01 \mathrm{~m}$. The contact patch boundary in the azimuth direction for $\bar{h}=0.01 \mathrm{~m}$ is $\theta_{p}=165.6^{\circ}$. Several terms are included in the series until the contact patch is well approximated and 
therefore equation (2.29) is satisfied. The radial component of the displacement field matches very well the prescribed deformation domain.

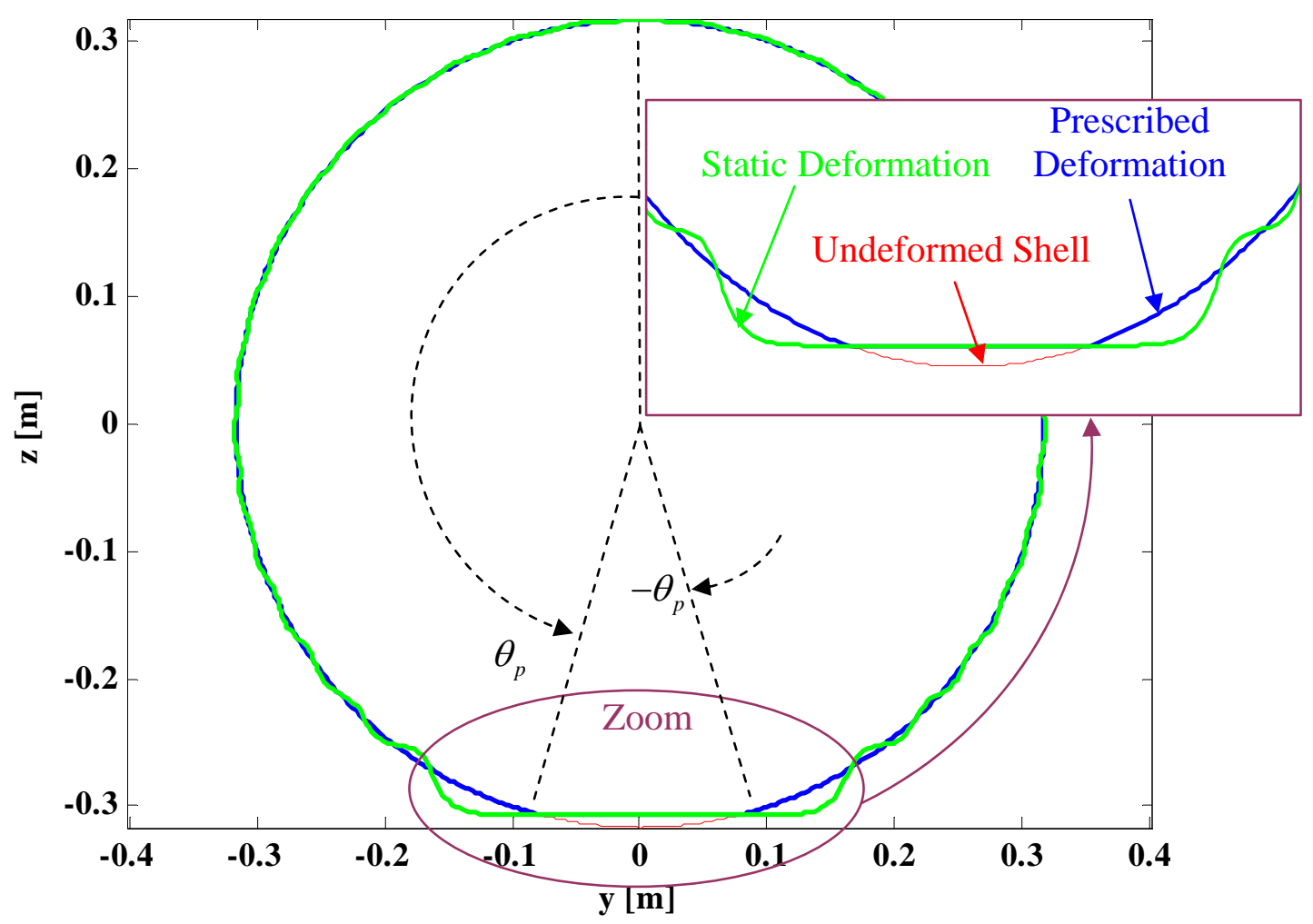

Figure 2.6: Radial displacement due to prescribed deformation at $x=\frac{L_{T}}{2}$ for tire size 195/65 R15 and $\bar{h}=0.01 m$.

In Figure 2.7 the radial static displacement field $w^{S}$ is shown. The upper figure represents the complete tire shell. The lower figure shows the contact patch region in detail. Therefore, the three-dimensional form of the contact patch modeled in this effort can be appreciated. The contact patch boundary in the axial directions is shown as well. 


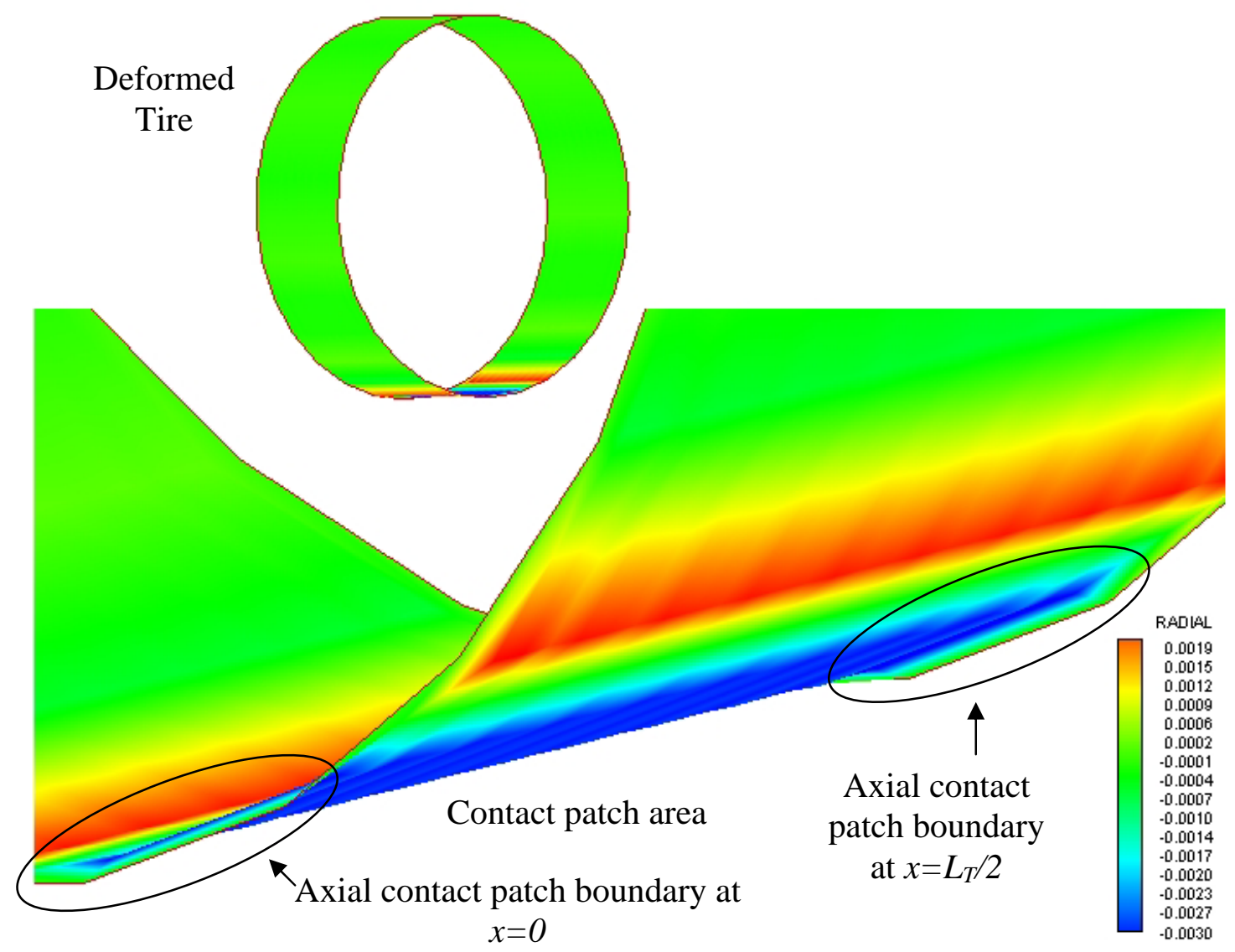

Figure 2.7: Radial displacement field $w^{S}[\mathrm{~m}]$ for tire size 195/65 R15 with $\bar{h}=0.01 \mathrm{~m}$.

In Figure 2.8, the static displacement predicted by the model is compared with experimental data collected by Yamauchi and Akiyoshi (2002) for a tire size 195/65 R15. The geometric parameters and material properties of the selected tire are given in Appendix A. This figure shows the radial displacement the "deformed tire", $w^{s}$, and the prescribed displacement function, $d(x, \theta)$, around the tire in the azimuth direction at the center line $x=L_{T} / 2$. The radial displacement, $w^{s}$, matches the contact patch very well. However, a very significant oscillation is observed and it is due to the $(1,8)$ mode. In order to compare the experimental data, an moving average of the static displacement away from the contact patch is also plotted (blue line). The average curve shows similar trend as the experiments. This suggests that the model overemphasizes the high order mode component not observed experimentally. Note that this $(1,8)$ mode component associated to the oscillation will not contribute to the spindle forces because it does not 
correspond to a "beam" mode with $n=1$ as described later in section 2.3. The predicted results shown in this figure are in relatively good agreement between the predicted and measured results in the contact patch area.

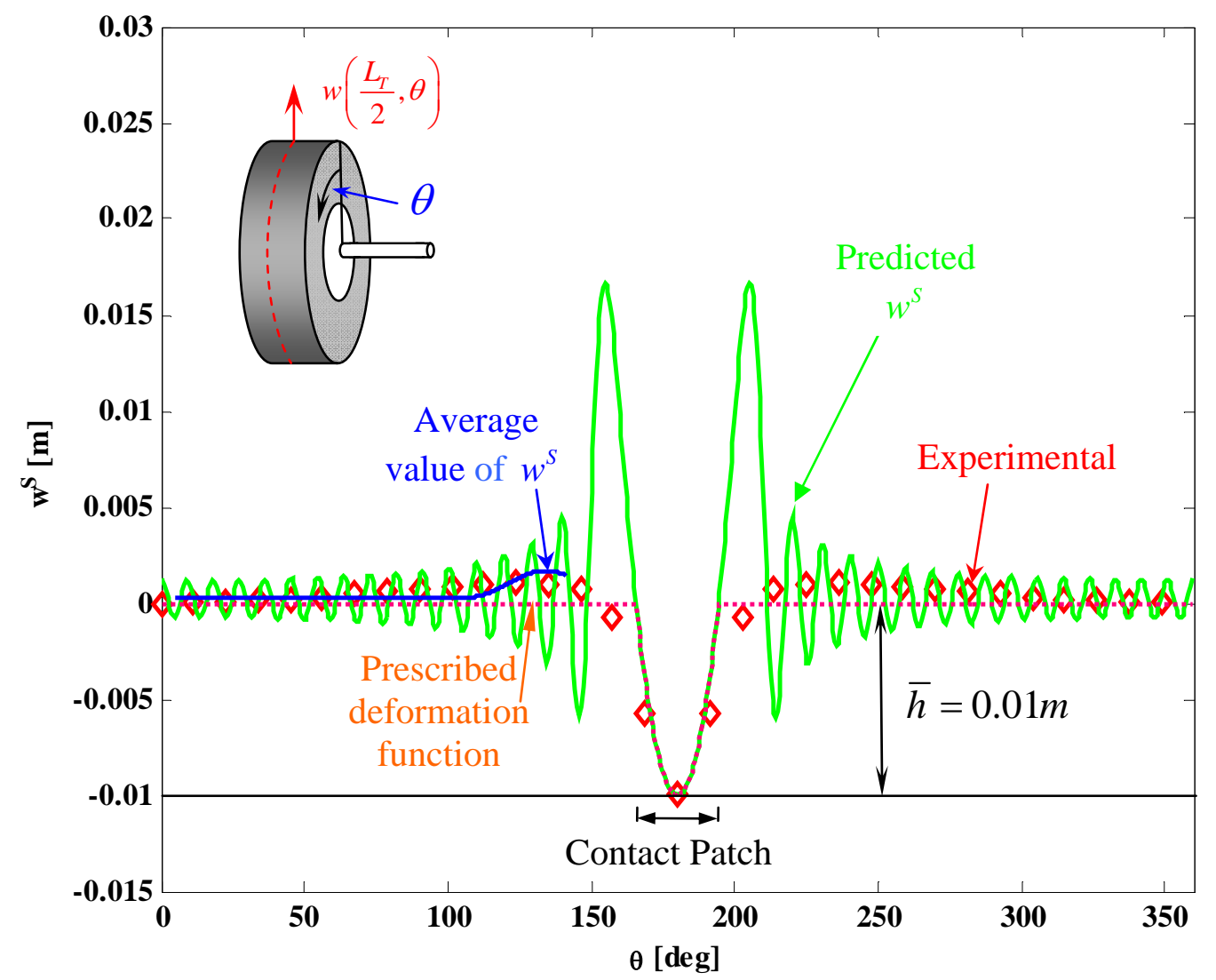

Figure 2.8: Radial displacement comparison between model and experimental data for tire size 195/65 R15 (Yamauchi and Akiyoshi, 2002).

The formulation up to this point allows to find the radial displacement component, $w^{s}$. That is the radial coefficient of the static displacement field as defined in equation (2.28). The other two components, i.e. axial $u^{s}$ and the tangential $v^{s}$ can now be determined. Since the displacement vector $\left\{u^{s}, v^{s}, w^{s}\right\}^{T}$ must satisfy the elasticity equations, it can be written that 


$$
\left[L_{c}\right]\left\{\begin{array}{l}
u^{s} \\
v^{s} \\
w^{s}
\end{array}\right\}=\left\{\begin{array}{l}
0 \\
0 \\
0
\end{array}\right\}
$$

Once again expressing the displacement vector as a linear combination of the mode shapes

$$
\left\{u^{s}\right\}=\sum_{m=1}^{M} \sum_{n=0}^{N}\left\{\begin{array}{l}
U_{m n}^{(3)} \cos \left(\frac{m \pi}{L_{T}} x\right) \cos (n \theta) \\
V_{m n}^{(3)} \sin \left(\frac{m \pi}{L_{T}} x\right) \sin (n \theta) \\
W_{m n}^{(3)} \sin \left(\frac{m \pi}{L_{T}} x\right) \cos (n \theta)
\end{array}\right\}
$$

and replacing into equation (2.32), it gives

$$
\left[\begin{array}{ccc}
-\lambda_{m}^{2}-\frac{\left(1-v^{2}\right)}{2} n^{2} & \frac{(1+v)}{2} n \lambda_{m} & v \lambda_{\mathrm{m}} \\
\frac{(1+v)}{2} n \lambda_{m} & (1-v) \lambda_{m}^{2}-n^{2} & -n \\
-v \lambda_{m} & n & 1+K\left(\lambda_{m}^{2}+n^{2}\right)^{2}
\end{array}\right]\left\{\begin{array}{l}
U_{m n}^{(3)} \\
V_{m n}^{(3)} \\
W_{m n}^{(3)}
\end{array}\right\}=\left\{\begin{array}{l}
0 \\
0 \\
0
\end{array}\right\}
$$

where the coefficients $W_{m n}^{(3)}$ are known from equation (2.31). The system of equations is then rearranged to give

$$
\left[\begin{array}{cc}
-\lambda_{m}^{2}-\frac{\left(1-v^{2}\right)}{2} n^{2} & \frac{(1+v)}{2} n \lambda_{m} \\
\frac{(1+v)}{2} n \lambda_{m} & (1-v) \lambda_{m}^{2}-n^{2} \\
-v \lambda_{m} & n
\end{array}\right]\left\{\begin{array}{l}
U_{m n}^{(3)} \\
V_{m n}^{(3)}
\end{array}\right\}=\left\{\begin{array}{c}
v \lambda_{\mathrm{m}} W_{m n}^{(3)} \\
-n W_{m n}^{(3)} \\
{\left[1+K\left(\lambda_{m}^{2}+n^{2}\right)^{2}\right] W_{m n}^{(3)}}
\end{array}\right\}
$$


This system of equations is solved by least squares, i.e. pseudo-inverse, for the coefficients $U_{m n}^{(3)}$ and $V_{m n}^{(3)}$. The coefficients $U_{m n}^{(3)}, V_{m n}^{(3)}$, and $W_{m n}^{(3)}$ are replaced back into equation (2.33) to give the complete deformed static displacement field.

Dynamic Displacement: The dynamic motion of the system is assumed to be produced by a harmonic vertical motion of the tire as shown in Figure 2.3c. The vertical motion of the tire translates into a time variation of the contact patch depth that can be expressed as

$$
h(t)=\bar{h}+\varepsilon e^{i \omega t}
$$

where $\varepsilon$ is the amplitude of the tire harmonic motion.

Since in this research effort the focus is in developing a general formulation, response spectra for a constant amplitude of the vertical motion $\varepsilon$ is determined. The response for a realistic vertical motion spectrum, i.e. characterized by the road properties, can then be used in conjunction with frequency response functions computed here.

The static displacement induced now by the time dependant contact patch depth, $h(t)$, oscillating with frequency $\omega$ induces inertial forces in the tire shell. The inertial effects will produce a dynamic response that needs to be superimposed to the static deformation. The time-varying static displacement can be easily obtained by replacing $\bar{h}$ with $h(t)$ in (2.31) to gives

$$
\left[I_{n r}(h(t))\right]\left\{W_{s n}^{(3)}\right\}=\left\{f_{s r}(h(t)\}\right.
$$

However, this matrix is time dependent and to find an explicit inverse is practically impossible. To solve for the time-dependant vector $\left\{W_{s n}^{(3)}\right\}$, small displacements are assumed i.e. $\varepsilon \ll \bar{h}$. Therefore, equation (2.31) is linearized with respect to $h(t)$ by 
using Taylor's series expansion evaluated around the equilibrium position $\bar{h}$. The expansion leads to

$$
\left\{W_{s n}^{(3)}\right\}=\left[I_{n r}(\bar{h})\right]^{-1}\left\{f_{s r}(\bar{h})\right\}+\left.\frac{d}{d h}\left\{\left[I_{n r}(h(t))\right]^{-1}\left\{f_{s r}(h(t))\right\}\right\}\right|_{\bar{h}}(h(t)-\bar{h})+O(\bar{h})^{2}(2.3
$$

The derivative term can be computed as

$$
\frac{d}{d h}\left(\left[I_{n r}(h)\right]^{-1}\left\{f_{s r}(h)\right\}\right)=\frac{d}{d h}\left(\left[I_{n r}(\bar{h})\right]^{-1}\right) \cdot\left\{f_{s r}(h)\right\}+\left[I_{n r}(h)\right]^{-1} \frac{d}{d h}\left(\left\{f_{s r}(h)\right\}\right)
$$

where the derivative of the matrix inverse $\frac{d}{d h}\left(\left[I_{n r}(h(t))\right]^{-1}\right)$ can be obtained from,

$$
\frac{d}{d h}\left(\left[I_{n r}(h(t))\right] \cdot\left[I_{n r}(h(t))\right]^{-1}\right)=\frac{d}{d h}([I]) \quad \forall t
$$

yielding

$$
\frac{d}{d h}\left(\left[I_{n r}(h(t))\right]^{-1}\right)=-\left[I_{n r}(h(t))\right]^{-1} \frac{d}{d h}\left(\left[I_{n r}(h(t))\right]\right)\left[I_{n r}(h(t))\right]^{-1}
$$

Substituting equation (2.39) and (2.41) into (2.38), the $\left\{W_{\text {sn }}\right\}$ are given by

$$
\left\{W_{s n}^{(3)}\right\}=\left[I_{n r}(\bar{h})\right]^{-1}\left\{f_{s r}(\bar{h})\right\}+\left[I_{n r}(\bar{h})\right]^{-1}\{D(\bar{h})\}(h(t)-\bar{h})
$$

where

$$
\{D(\bar{h})\}=\left.\frac{d}{d h}\left(\left\{f_{s r}(h)\right\}\right)\right|_{\bar{h}}-\left.\frac{d}{d h}\left(\left[I_{n r}(h)\right]\right)\right|_{\bar{h}}\left[I_{n r}(\bar{h})\right]^{-1}\left\{f_{s r}(\bar{h})\right\}
$$


Since the contact patch depth has a harmonic motion with amplitude $\varepsilon$, the vector $\left\{W_{s n}^{(3)}\right\}$ can be written as the sum of a static deformation $\left\{\bar{W}_{s n}^{(3)}\right\}$ due to $\bar{h}$ and an oscillating part $\left\{\tilde{W}_{s n}^{(3)}\right\}$ due to the harmonic oscillation around $\bar{h}$. That is

$$
\left\{W_{s n}^{(3)}\right\}=\left\{\bar{W}_{s n}^{(3)}\right\}+\left\{\tilde{W}_{s n}^{(3)}\right\} e^{i \omega t}=\left[I_{n r}(\bar{h})\right]^{-1}\left\{f_{s r}(\bar{h})\right\}+\left[I_{n r}(\bar{h})\right]^{-1}\{D(\bar{h})\} \varepsilon e^{i \omega t}
$$

The axial and tangential displacement components due to the harmonic component of the static displacement can again be computed using equation (2.43). Thus, the complete static displacement field and the time-dependant part are fully defined, i.e. $\left\{u^{s}, v^{s}, w^{s}\right\}^{T}=\left\{\bar{u}^{s}, \bar{v}^{s}, \bar{w}^{s}\right\}^{T}+\left\{\tilde{u}^{s}, \tilde{v}^{s}, \tilde{w}^{s}\right\}^{T}$. It is important to note that it was found that the axial and tangential displacement components can be neglected since their contribution to the response is insignificant.

The dynamic response due to the oscillating component of the static displacement field, $\left\{\tilde{u}^{s}, \tilde{v}^{s}, \tilde{w}^{s}\right\}^{T}$, can now be determined. Applying the shell Donnell-Mushtari operator, $\left[L_{D-M}\right]$, to the total displacement vector $\left\{u^{t}\right\}$ yields

$$
\left(\left[L_{c}\right]-\Omega^{2}[I]\right)\left\{\begin{array}{l}
u^{t} \\
v^{t} \\
w^{t}
\end{array}\right\}=\{0\}
$$

where the total displacement vector is the contribution of the dynamic displacement due to the shell inertia and the time-dependant part of the static displacement due to the contact patch oscillation around $\bar{h}$. That is 


$$
\left\{\begin{array}{l}
u^{t} \\
v^{t} \\
w^{t}
\end{array}\right\}=\left\{\begin{array}{l}
u^{d} \\
v^{d} \\
w^{d}
\end{array}\right\}+\left\{\begin{array}{l}
\tilde{u}^{s} \\
\tilde{v}^{s} \\
\tilde{w}^{s}
\end{array}\right\}
$$

where

$$
\left\{\begin{array}{l}
\tilde{u}^{s} \\
\tilde{v}^{s} \\
\tilde{w}^{s}
\end{array}\right\}=\left\{\begin{array}{c}
0 \\
0 \\
\sum_{s=1}^{S} \sum_{n=0}^{N} W_{s n}^{(3)} \sin \left(\frac{s \pi}{L_{T}} x\right) \cos (n \theta)
\end{array}\right\}
$$

That is the axial $\tilde{u}^{s}$ and tangential $\tilde{v}^{s}$ displacements are neglected.

Thus, equation (2.45) can be written as

$$
\left(\left[L_{c}\right]-\Omega^{2}[I]\right)\left\{\begin{array}{l}
u^{d} \\
v^{d} \\
w^{d}
\end{array}\right\}=-\left(\left[L_{c}\right]-\Omega^{2}[I]\right)\left\{\begin{array}{l}
\tilde{u}^{s} \\
\tilde{v}^{s} \\
\tilde{w}^{s}
\end{array}\right\}=\left\{\begin{array}{l}
f_{u} \\
f_{v} \\
f_{w}
\end{array}\right\}
$$

where the right hand side of equation (2.48) can be recognized as a loading term due to the oscillating static displacement.

The procedure to solve the system of differential equation (2.48) is perform in the same manner than for the case of the "free tire" model in section 2.1. Then, the response of the "deformed tire” model is then used to compute the forces at the spindle. The modeling of the forces at the spindle is the topic presented in the next section. 


\subsection{FORCES TRANSMITTED TO THE “SPINDLE”}

The objective of this section is to formulate the expression for the forces transmitted to the vehicle spindle due to the vibration of the tire system. Then, these forces can be used as an input in FE models of the car structure.

The previous sections described the steps to solve for the structural modal amplitudes. The approach to compute spindle force and moment is to first separate the elastic outer shell from the rest of the system and find the boundary forces per unit length of the outer flexible shell; i.e. the normal force $T_{X}$ and tangential $S_{X}$ as illustrated in Figure 2.9. Finally, the boundary forces are translated from the boundary to the spindle. This process results in a vertical force $\{F\}_{\text {Spindle }}^{\text {Total }}$ and a horizontal moment $\{M\}_{\text {Spindle }}^{\text {Total }}$ as shown in Figure 2.9. These two forces keep the system in static equilibrium.

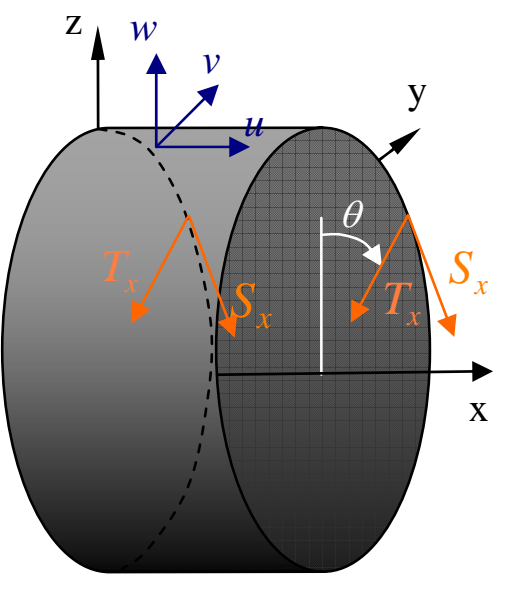

Elastic shell

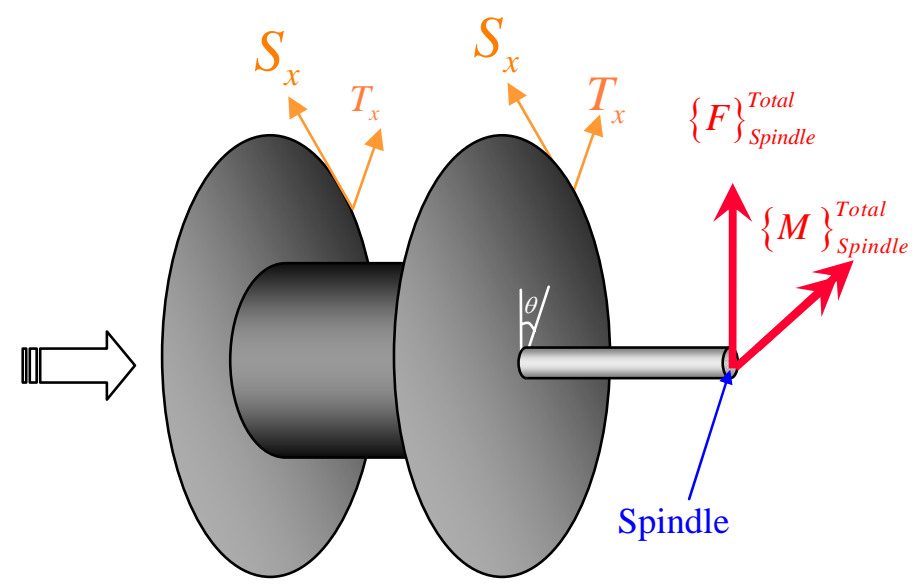

Rigid system

Figure 2.9: Spindle force and moment.

To compute the normal $T_{X}$ and tangential $S_{X}$ forces on the boundary of the elastic shell, a variational approach is used and shown in Appendix C. Then, the expressions of the resultants forces at the boundary are easily computed. 
Using the results obtained in Appendix C, for the case of a circular cylindrical shell as the tire is modeled the following parameters must be set

$$
\begin{array}{rlrl}
\alpha=\frac{x}{a} & \beta=\theta \\
A=a & B=a \\
R_{\alpha}=\infty & R_{\beta}=a
\end{array}
$$

Consider a closed circular cylindrical shell of finite length $L$, which satisfies the boundary conditions at $x=0, L_{T}$ given in the Appendix $\mathrm{C}$ as follow

$$
\begin{array}{ccc}
N_{x}=0 & \Rightarrow \mathrm{u} \neq 0 \\
N_{x \theta}+\frac{M_{x \theta}}{a}=S_{x} & \Rightarrow \quad v=0 \\
Q_{x}+\frac{1}{a} \frac{\partial M_{x \theta}}{\partial \theta}=T_{x} & \Rightarrow w=0
\end{array}
$$

where $S_{x}$ represent a tangential force per unit length, and $T_{x}$ is a normal force per unit length. These forces are then translated to the spindle location and integrated over the shell boundary, i.e. $0 \leq \theta \leq 2 \pi$. The final equations for the forces are developed now in the next paragraphs using the approximated shell theory given by Donnell-Mushtari -Vlasov.

The strain-displacement relationship using Kirchhoff ${ }^{2}$ hypothesis in matrix form can be written as, Leissa (1993),

\footnotetext{
${ }^{2}$ Gustav Robert Kirchhoff

Born: 12 March, 1824, Königsberg, East Prussia (now Kaliningrad, Russia)

Died: 17 Oct 1887 in Berlin, Germany

Kirchhoff was a student of Gauss. He taught at Berlin (an unpaid post) from 1847, then at Breslau. In 1854 he was appointed professor of physics at Heidelberg where he collaborated with Bunsen.

He was a physicist who made important contributions to the theory of circuits using topology and to elasticity. Kirchhoff's Laws, announced in 1854, allow calculation of currents, voltages and resistances of electrical circuits extending the work of Ohm. His work on black body radiation was fundamental in the development of quantum theory.
} 


$$
\left\{\begin{array}{l}
\varepsilon_{x} \\
\varepsilon_{\theta} \\
\varepsilon_{x \theta}
\end{array}\right\}=\left[\begin{array}{ccc}
\frac{\partial}{\partial x} & 0 & 0 \\
0 & \frac{1}{a} \frac{\partial}{\partial \theta} & \frac{1}{a} \\
\frac{1}{a} \frac{\partial}{\partial \theta} & \frac{\partial}{\partial x} & 0
\end{array}\right] \cdot\left\{\begin{array}{l}
u \\
v \\
w
\end{array}\right\}=\left[D_{1}\right] \cdot\left\{\begin{array}{l}
u \\
v \\
w
\end{array}\right\}
$$

Replacing the displacement in terms of the modal amplitudes (with or without fluid loading effects), the strain becomes

$$
\{\varepsilon\}=\left[D_{1}\right] \sum_{m=1}^{M} \sum_{n=0}^{N}\left[\Phi_{m n}\right]\left\{A_{m n}\right\}
$$

The resultant forces at the boundary in matrix notation becomes

$$
\left\{\begin{array}{l}
N_{x} \\
N_{\theta} \\
N_{x \theta}
\end{array}\right\}=\left[\begin{array}{ccc}
\frac{E h}{\left(1-v^{2}\right)} & \frac{E h v}{\left(1-v^{2}\right)} & 0 \\
\frac{v E h}{\left(1-v^{2}\right)} & \frac{E h}{\left(1-v^{2}\right)} & 0 \\
0 & 0 & \frac{E h}{2(1+v)}
\end{array}\right] \cdot\left\{\begin{array}{c}
\varepsilon_{x} \\
\varepsilon_{\theta} \\
\varepsilon_{x \theta}
\end{array}\right\}
$$

Once again in terms of the modal amplitudes is

$$
[N]=\left[C_{1}\right] \cdot[\varepsilon]=\left[C_{1}\right] \cdot\left[D_{1}\right] \sum_{m=1}^{M} \sum_{n=0}^{N}[\Phi]\left\{A_{m n}\right\}
$$

Using the same approach, the resultant moments at the boundary becomes

His work on spectrum analysis led on to a study of the composition of light from the Sun. Kirchhoff was the first to explain the dark lines in the Sun's spectrum as caused by absorbsion of particular wavelengths as the light passes through a gas. This started a new era in astronomy. In 1875 he was appointed to the chair of mathematical physics at Berlin. Disability meant he had to spend much of his life on crutches or in a wheelchair. His best known work is the four volume masterpiece Vorlesungen über mathematische Physik (1876-94).

(Source: http://www.acmi.net.au/AIC/KIRCHHOFF BIO.html) 


$$
\left\{\begin{array}{l}
K_{x} \\
K_{\theta} \\
\tau
\end{array}\right\}=\left[\begin{array}{ccc}
0 & 0 & -\frac{\partial^{2}}{\partial x^{2}} \\
0 & 0 & -\frac{1}{a^{2}} \frac{\partial^{2}}{\partial \theta^{2}} \\
0 & 0 & -\frac{2}{a} \frac{\partial^{2}}{\partial \theta \partial x}
\end{array}\right] \cdot\left\{\begin{array}{l}
u \\
v \\
w
\end{array}\right\}=\left[D_{2}\right] \cdot\left\{\begin{array}{l}
u \\
v \\
w
\end{array}\right\}
$$

The change in curvature can be expressed in terms of a linear combination of the modes

$$
\{K\}=\left[D_{2}\right] \sum_{m=1}^{M} \sum_{n=0}^{N}\left[\Phi_{m n}\right]\left\{A_{m n}\right\}
$$

that results in the resultant moments as

$$
\left\{\begin{array}{l}
M_{x} \\
M_{\theta} \\
M_{x \theta}
\end{array}\right\}=\left[\begin{array}{ccc}
\frac{E h^{3}}{12\left(1-v^{2}\right)} & \frac{E h^{3} v}{12\left(1-v^{2}\right)} & 0 \\
\frac{E h^{3} v}{12\left(1-v^{2}\right)} & \frac{E h^{3}}{12\left(1-v^{2}\right)} & 0 \\
0 & 0 & \frac{E h^{3}}{24(1+v)}
\end{array}\right] \cdot\left\{\begin{array}{l}
\kappa_{x} \\
\kappa_{\theta} \\
\tau
\end{array}\right\}
$$

In matrix notation equation (2.56) can be rewrite:

$$
\{M\}=\left[C_{2}\right] \cdot\{K\}=\left[C_{2}\right] \cdot\left[D_{2}\right] \sum_{m=1}^{M} \sum_{n=0}^{N}\left[\Phi_{m n}\right]\left\{A_{m n}\right\}
$$

and the 


$$
\left\{\begin{array}{l}
Q_{x} \\
Q_{\theta} \\
0
\end{array}\right\}=\left[\begin{array}{ccc}
\frac{E h^{3}}{12\left(1-v^{2}\right)} \frac{\partial}{\partial x} & \frac{E h^{3} v}{12\left(1-v^{2}\right)} \frac{\partial}{\partial x} & 0 \\
\frac{E h^{3} v}{12\left(1-v^{2}\right)} \frac{\partial}{\partial \theta} & \frac{E h^{3}}{12\left(1-v^{2}\right)} \frac{\partial}{\partial \theta} & 0 \\
0 & 0 & 0
\end{array}\right] \cdot\left\{\begin{array}{l}
\kappa_{x} \\
\kappa_{\theta} \\
\tau
\end{array}\right\}
$$

thus,

$$
\{Q\}=\left[C_{3}\right] \cdot\{K\}=\left[C_{3}\right] \cdot\left[D_{2}\right] \sum_{m=1}^{M} \sum_{n=0}^{N}\left[\Phi_{m n}\right]\left\{A_{m n}\right\}
$$

Then, applying the "shear diaphragms” or simply supported boundary conditions

$$
w=M_{x}=N_{x}=v=0 \text { at } x=0, L
$$

the tangential and shearing forces become

$$
\left\{\begin{array}{l}
T_{x} \\
S_{x} \\
0
\end{array}\right\}=\left[\begin{array}{ccc}
0 & 0 & \frac{1}{a} \frac{\partial}{\partial \theta} \\
0 & 0 & \frac{1}{a} \\
0 & 0 & 0
\end{array}\right]\left\{\begin{array}{l}
M_{x} \\
M_{\theta} \\
M_{x \theta}
\end{array}\right\}+\left[\begin{array}{ccc}
0 & 0 & 0 \\
0 & 0 & 1 \\
0 & 0 & 0
\end{array}\right] \cdot\left\{\begin{array}{l}
N_{x} \\
N_{\theta} \\
N_{x \theta}
\end{array}\right\}+\left[\begin{array}{ccc}
1 & 0 & 0 \\
0 & 0 & 0 \\
0 & 0 & 0
\end{array}\right]\left\{\begin{array}{l}
Q_{x} \\
Q_{\theta} \\
Q_{x \theta}
\end{array}\right\}
$$

and in terms of the modal amplitudes results in

$$
\begin{aligned}
\left\{F_{x}\right\}= & {\left[B_{2}\right] \cdot[M]+\left[B_{1}\right] \cdot[N]+\left[B_{3}\right] \cdot[Q] } \\
= & {\left[B_{2}\right]\left[C_{2}\right]\left[D_{2}\right] C \sum_{m=1}^{M} \sum_{n=0}^{N}\left[\Phi_{m n}\right]\left\{A_{m n}\right\}+\left[B_{1}\right]\left[C_{1}\right]\left[D_{1}\right] C \sum_{m=1}^{M} \sum_{n=0}^{N}\left[\Phi_{m n}\right]\left\{A_{m n}\right\}^{(2.6} } \\
& +\left[B_{3}\right]\left[C_{3}\right] \cdot\left[D_{2}\right] C \sum_{m=1}^{M} \sum_{n=0}^{N}\left[\Phi_{m n}\right]\left\{A_{m n}\right\}
\end{aligned}
$$

Therefore, 


$$
\left\{F_{x}\right\}=\left(\left[B_{2}\right]\left[C_{2}\right]\left[D_{2}\right]+\left[B_{1}\right]\left[C_{1}\right]\left[D_{1}\right]+\left[B_{3}\right]\left[C_{3}\right] \cdot\left[D_{2}\right]\right) \sum_{m=1}^{M} \sum_{n=0}^{N}\left[\Phi_{m n}\right]\left\{A_{m n}\right\}
$$

where

$$
\left\{F_{x}\right\}=\left\{T_{x}, S_{x}, 0\right\}^{T}
$$

Next the boundary forces are projected into the Cartesian coordinates to give

$$
\left\{\begin{array}{l}
F_{y} \\
F_{z} \\
0
\end{array}\right\}=\left[\begin{array}{ccc}
\cos (\theta) & -\sin (\theta) & 0 \\
\sin (\theta) & \cos (\theta) & 0 \\
0 & 0 & 1
\end{array}\right]\left\{\begin{array}{l}
T_{x} \\
S_{x} \\
0
\end{array}\right\}
$$

or

$$
\{F\}=[R]\left\{F_{x}\right\}=[R]\left(\left[B_{2}\right]\left[C_{2}\right]\left[D_{2}\right]+\left[B_{1}\right]\left[C_{1}\right]\left[D_{1}\right]+\left[B_{3}\right]\left[C_{3}\right] \cdot\left[D_{2}\right]\right) \sum_{m=1}^{M} \sum_{n=0}^{N}[\Phi]\left\{A_{m n}\right\}
$$

where

$$
\{F\}=\left\{F_{y}, F_{z}, 0\right\}
$$

The next step is to translate the forces per unit length from the shell left and right edges to the spindle point, located at the end on the car axis. The approach is represented in Figure 2.9 .

The procedure yields a force

$$
\{F\}_{\text {Spindle }}=\{F\}_{\text {Left }, x=0}+\{F\}_{\text {Rigth }, x=L}
$$

and a moment

$$
\{M\}_{\text {Spindle }}=\left\{r^{s-L e f t}\right\} \times\{F\}_{\text {Left }, x=0}+\left\{r^{s-\text { Rigtht }}\right\} \times\{F\}_{\text {Rigth }, x=L}
$$


Finally, the spindle forces and moments due to the right and left boundaries are obtained by integrating along the shell edge

$$
\{F\}_{\text {Spindle }}^{\text {Total }}=\int_{0}^{2 \pi}\left\{\{F\}_{\text {Leff, } x=0}+\{F\}_{\text {Right }, x=L_{T}}\right\} a d \theta
$$

and

$$
\{M\}_{\text {Spindle }}^{\text {Total }}=\int_{0}^{2 \pi}\left\{\left\{r^{s-\text { Left }}\right\} \times\{F\}_{\text {Left }, x=0}+\left\{r^{s-\text { Righth }}\right\} \times\{F\}_{\text {Right }, x=L_{T}}\right\} \operatorname{ad} \theta
$$

The final expression for the force at the spindle is

$$
\begin{aligned}
\{F\}_{\text {Spindle }}^{\text {Total }}= & \sum_{m=1}^{M}\left(\frac { [ 1 + ( - 1 ) ^ { m } ] E h \pi } { 1 2 a L _ { T } ^ { 3 } ( 1 + v ) ( 1 + v ^ { 2 } ) } \left\{h^{2} L_{T}^{2} m \pi \bar{W}_{m 1}[1-(v-2) v]+6 a L_{T}^{3} \bar{U}_{m 1}\left(1+v^{2}\right)\right.\right. \\
& \left.+a^{2} m \pi\left[h^{2} m^{2} \pi^{2} \bar{W}_{m 1}(1+v)-6 L_{T}^{2} \bar{V}_{m 1}\left(1+v^{2}\right)\right]\right) \hat{k}
\end{aligned}
$$

and

$$
\begin{aligned}
\{M\}_{\text {Spindle }}^{\text {Total }}= & \sum_{m=1}^{M}\left(\frac { - E h \pi [ d + ( - 1 ) ^ { m } d + L _ { T } ] } { 1 2 a L _ { T } ^ { 3 } ( 1 + v ) ( 1 + v ^ { 2 } ) } \left\{h^{2} L_{T}^{2} m \pi \bar{W}_{m 1}[1-(v-2) v]+6 a L_{T}^{3} \bar{U}_{m 1}\left(1+v^{2}\right)\right.\right. \\
& \left.+a^{2} m \pi\left[h^{2} m^{2} \pi^{2} \bar{W}_{m 1}(1+v)-6 L_{T}^{2} \bar{V}_{m 1}\left(1+v^{2}\right)\right]\right) \hat{j}
\end{aligned}
$$

Where

$$
\begin{aligned}
& \bar{U}_{m n}=U_{m n}^{(1)} A_{m n}^{(1)}+U_{m n}^{(2)} A_{m n}^{(2)}+U_{m n}^{(3)} A_{m n}^{(3)} \\
& \bar{V}_{m n}=V_{m n}^{(1)} A_{m n}^{(1)}+V_{m n}^{(2)} A_{m n}^{(2)}+V_{m n}^{(3)} A_{m n}^{(3)} \\
& \bar{W}_{m n}=W_{m n}^{(1)} A_{m n}^{(1)}+W_{m n}^{(2)} A_{m n}^{(2)}+W_{m n}^{(3)} A_{m n}^{(3)}
\end{aligned}
$$




\subsection{FREE TIRE NUMERICAL SIMULATIONS}

In order to illustrate the models developed in this chapter, the 195/65 R15 tire size was used for the simulations. The geometric parameters and material properties of the selected tire are given in the Appendix A and in Table 2.1. The material properties of the tire were provided by Dimitri Tsihlas from Michelin North America (Tsihlas, 2001). Table 2.2 shows the natural frequencies for the tire structure over the $[0-400 \mathrm{~Hz}]$ frequency range. The dominant displacement component is also indicated in the Table.

Table 2.1: Tire’s material and geometrical parameters

\begin{tabular}{|c|c|}
\hline Parameter & Description \\
\hline$L_{T}=0.195 m(7.68$ in $)$ & Tire Width \\
\hline $\boldsymbol{a}=\mathbf{0 . 3 1 8} \boldsymbol{m}(12.52 \mathrm{in})$ & Outer Radius \\
\hline $\boldsymbol{b}=\mathbf{0 . 1 9 1 m}(7.52 \mathrm{in})$ & Inner Radius \\
\hline $\boldsymbol{h}_{\text {Shell }}=0.015 \boldsymbol{m}(0.59 \mathrm{in})$ & Thickness \\
\hline $\boldsymbol{d}=\mathbf{0 . 0 5} \boldsymbol{m}(1.97 \mathrm{in})$ & Spindle length \\
\hline$E=75 \times 10^{6} \mathrm{~N} / \mathrm{m}^{2}$ & Young's Modulus \\
\hline$\rho=1350 \mathrm{~kg} / \mathrm{m}^{3}$ & Material Density \\
\hline$\eta=0.03$ & Loss Factor \\
\hline$v=0.4$ & Poisson ratio \\
\hline$F=1$ & Force magnitude for the "free tire" \\
\hline
\end{tabular}


Table 2.2: Tire structure natural frequencies between 0 and $400 \mathrm{~Hz}$

\begin{tabular}{|c|c|c|c|}
\hline $\mathbf{m}$ & $\mathbf{n}$ & frequency [Hz] & Mode Type \\
\hline 1 & 4 & 105.9 & Radial \\
\hline 1 & 3 & 106.9 & Radial \\
\hline 1 & 5 & 112.8 & Radial \\
\hline 1 & 2 & 113.4 & Radial \\
\hline 1 & 1 & 121.0 & Radial \\
\hline 1 & 6 & 127.4 & Radial \\
\hline 1 & 7 & 148.6 & Radial \\
\hline 1 & 8 & 175.3 & Radial \\
\hline 1 & 9 & 206.9 & Radial \\
\hline 2 & 0 & 231.2 & Radial \\
\hline 2 & 1 & 232.3 & Radial \\
\hline 2 & 2 & 235.7 & Radial \\
\hline 2 & 3 & 242.0 & Radial \\
\hline 1 & 10 & 242.9 & Radial \\
\hline 2 & 4 & 251.5 & Radial \\
\hline 2 & 5 & 264.9 & Radial \\
\hline 2 & 6 & 282.5 & Radial \\
\hline 1 & 11 & 283.0 & Radial \\
\hline 2 & 7 & 304.4 & Radial \\
\hline 1 & 12 & 327.1 & Radial \\
\hline 2 & 8 & 330.8 & Radial \\
\hline 1 & 1 & 356.2 & Tangential \\
\hline 2 & 9 & 361.6 & Radial \\
\hline 1 & 13 & 375.2 & Radial \\
\hline 1 & 2 & 377.4 & Tangential \\
\hline 2 & 10 & 396.7 & Radial \\
\hline
\end{tabular}

The input harmonic force $f_{w}=F \cdot \delta\left(\theta-\theta_{o}\right) \cdot \delta\left(x-x_{f}\right) \cdot e^{i \omega t}$ is placed at $\theta_{o}=0$ and $x_{f}=\frac{L_{T}}{2}$.

For the sake of completeness, the modal amplitude response for the fundamental mode is shown in Figure 2.10. 


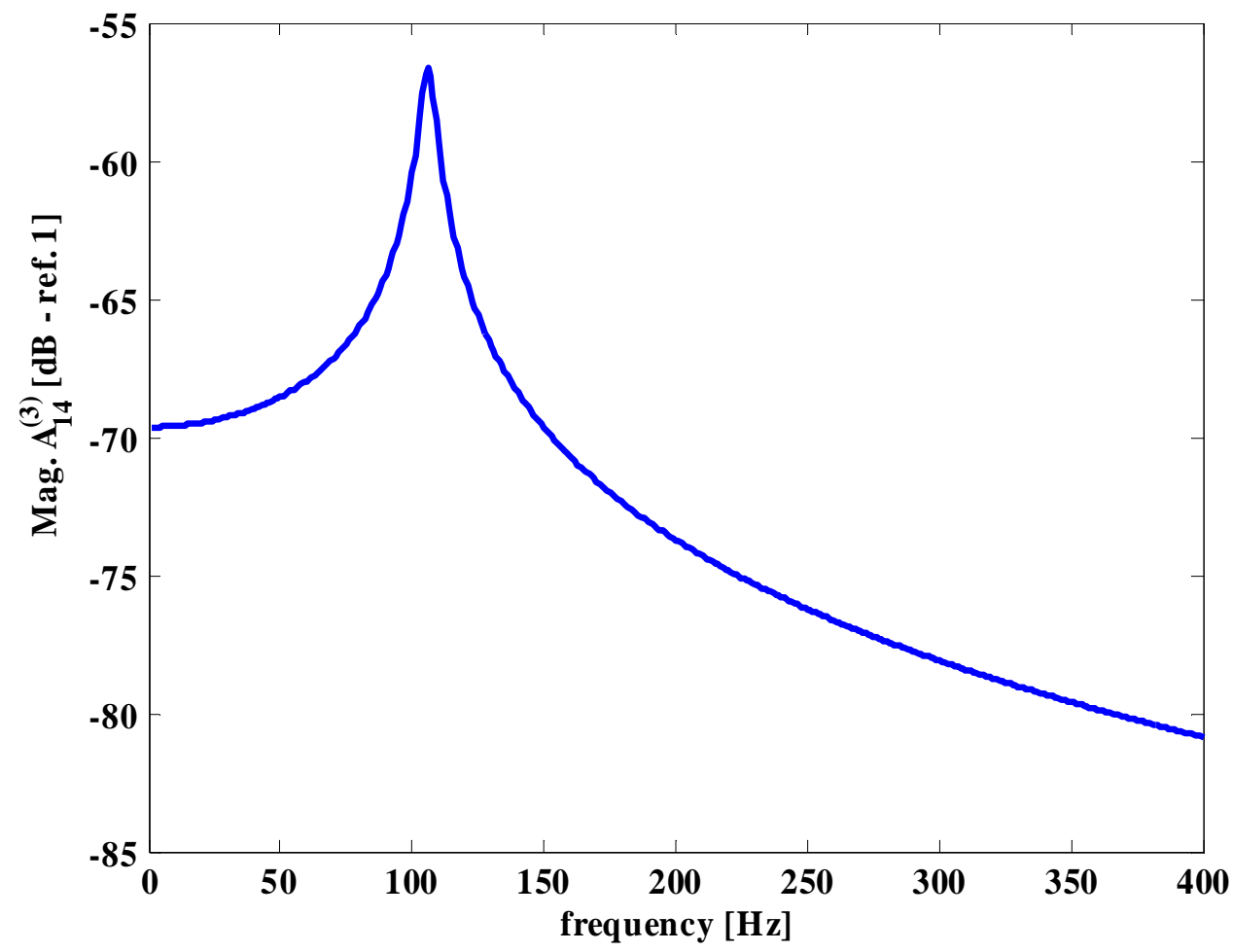

Figure 2.10: Structural Modal Amplitude for mode $\Phi_{14}^{(1)}$ with resonance at $105.9 \mathrm{~Hz}$.

The simulation was performed using the 25 modes corresponding to $n=0, . ., 4$ and $m=1, . .5$, and the spindle forces computed. The spindle vertical force and the horizontal moment were normalized by the input force. Figure 2.11 and 2.12 show the vertical force and horizontal moment at the spindle, respectively. Though the tire structure is a modally rich system, the forces are dominated by the contribution of only two structural modes, i.e. the radial and tangential $(1,1)$ modes. From the spindle force computation, it is seen that all forces and moments are zero for $n \neq 1$ (Greenspon, 1958), i.e. only "beam” type or $n=1$ shell modes transmit forces to the spindle. The motion corresponding to the case $n \neq 1$ are the so called "lobar" vibrations of the cylinder, and there is not resultant force. This means that the centroid of the section moves in the transversal direction $y$ during the vibration. The vibration produces a reaction at the support and a resultant bending of the entire cylindrical tire. Then, these forces are transmitted by a rigid spindle to the rest of the vehicle. 


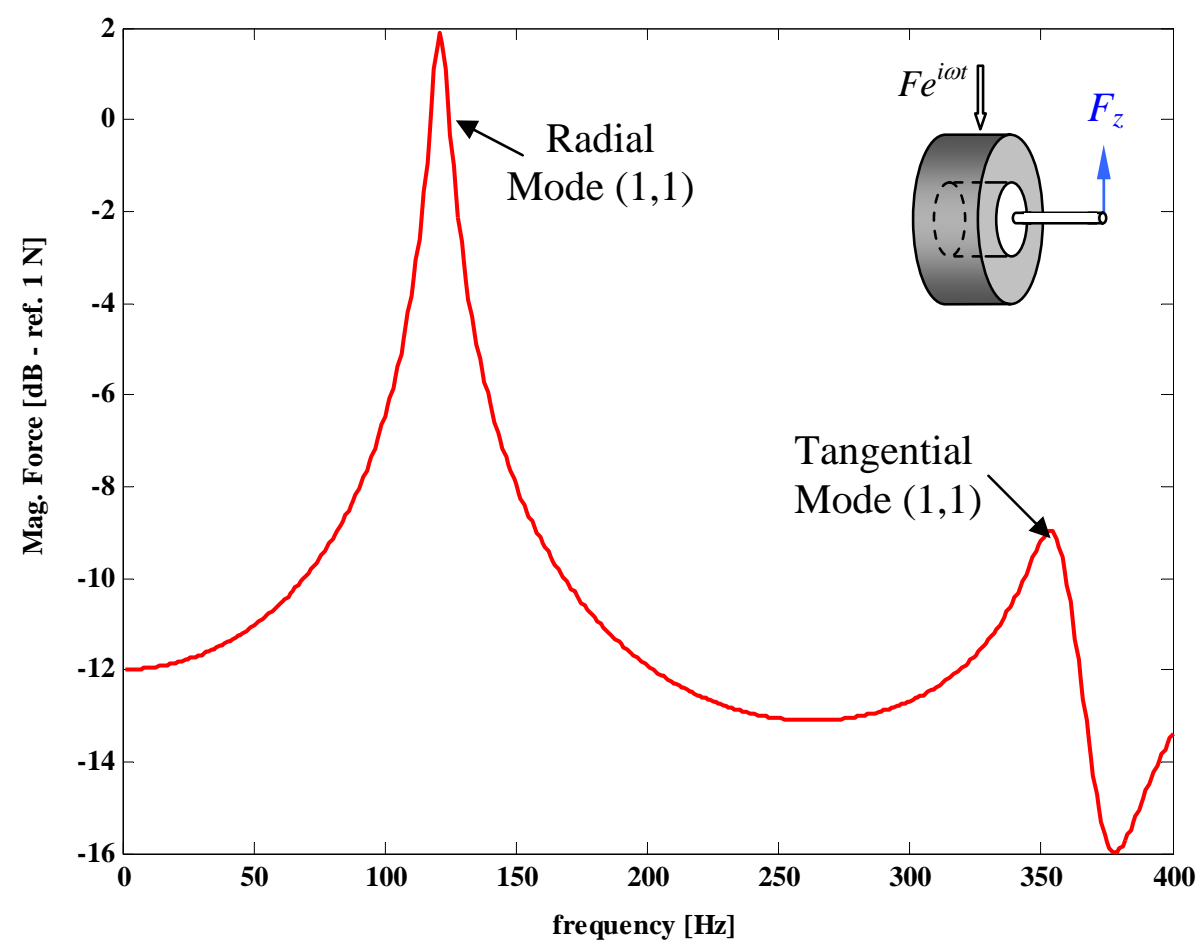

Figure 2.11: Vertical force $F_{z}$ at the spindle.

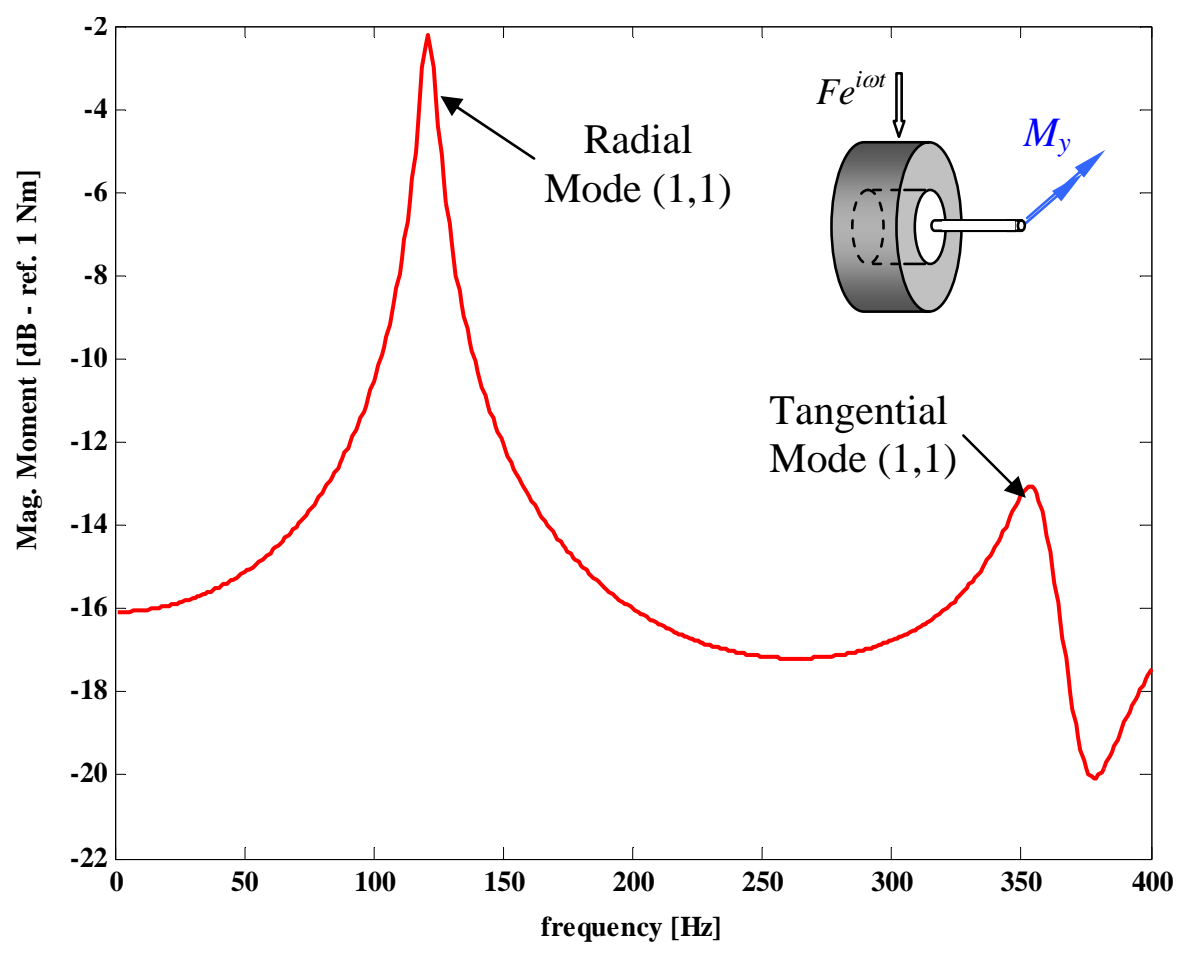

Figure 2.12: Horizontal moment $\mathrm{M}_{\mathrm{y}}$ at the spindle. 
The radial displacement at the external force location of the tire is computed and shown in Figure 2.13. The radial displacement is dominated by the radial modes $(1,3)$ and $(1,0)$.

To validate the model, the predicted point accelerance for the free tire is compared to experimental data in Figure 2.14. To compare experimental and analytical results a gain was applied upon the analytical results. The gain factor was selected in such a way to match the experimental with the analytical acoustic cavity resonance (see section 3.4). The same gain value is applied to all the results presented in this dissertation. The experimental data corresponds to the point accelerance shown in the work by Yamauchi and Akiyoshi (2002) for the tire 195/65 R15.

The experimental data plotted in the figure shows the contribution of the acoustic cavity resonance at $238 \mathrm{~Hz}$. The structure resonances at 107 and $142 \mathrm{~Hz}$ correspond to the radial $(1,3)$ and radial $(1,0)$ modes, respectively. The results in this figure show that the free tire model predicts very well the experimental data except around the acoustic cavity resonance which was not yet included in the model. Thus, this comparison validates the modeling approach. Additional comparisons will be presented later in this research effort to further validate the model. 


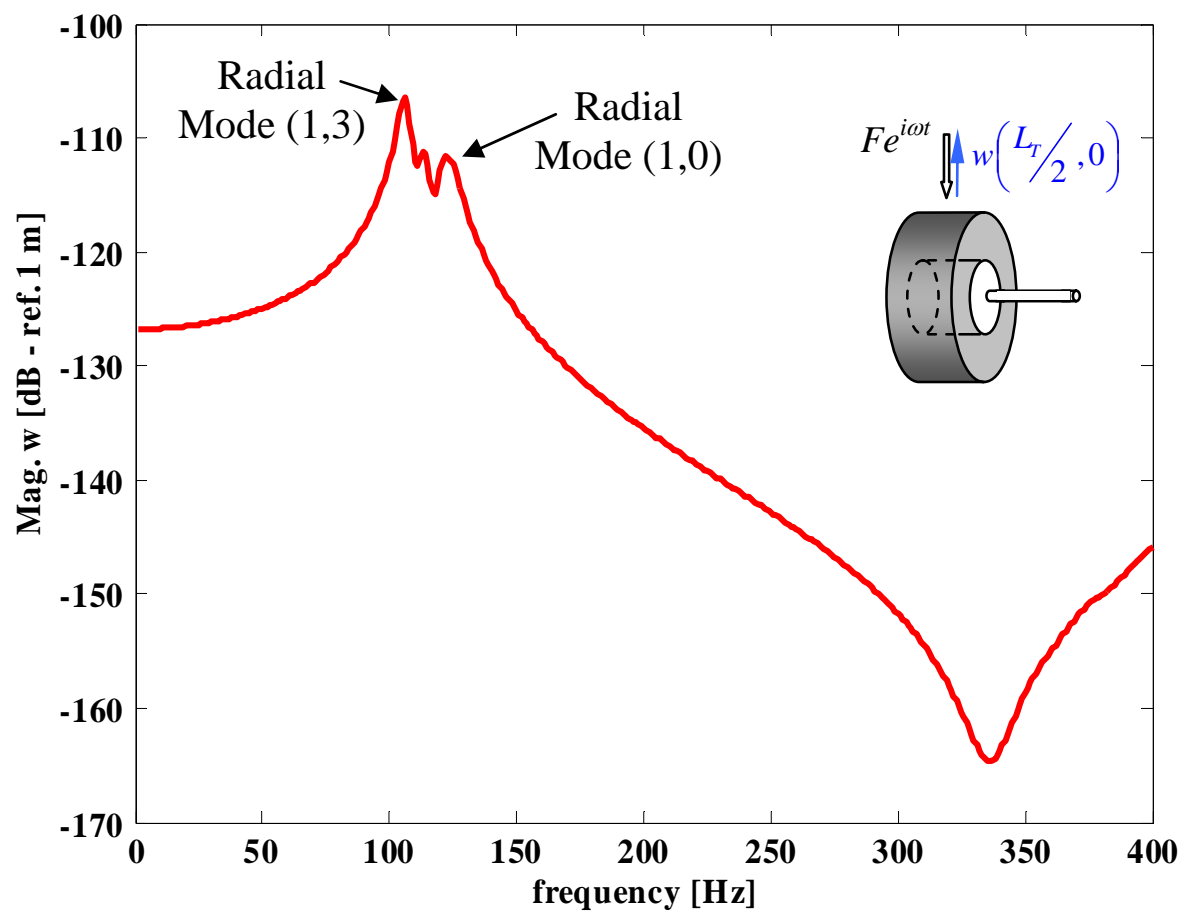

Figure 2.13: Radial displacement for the in vacuo free tire model at the external force location.

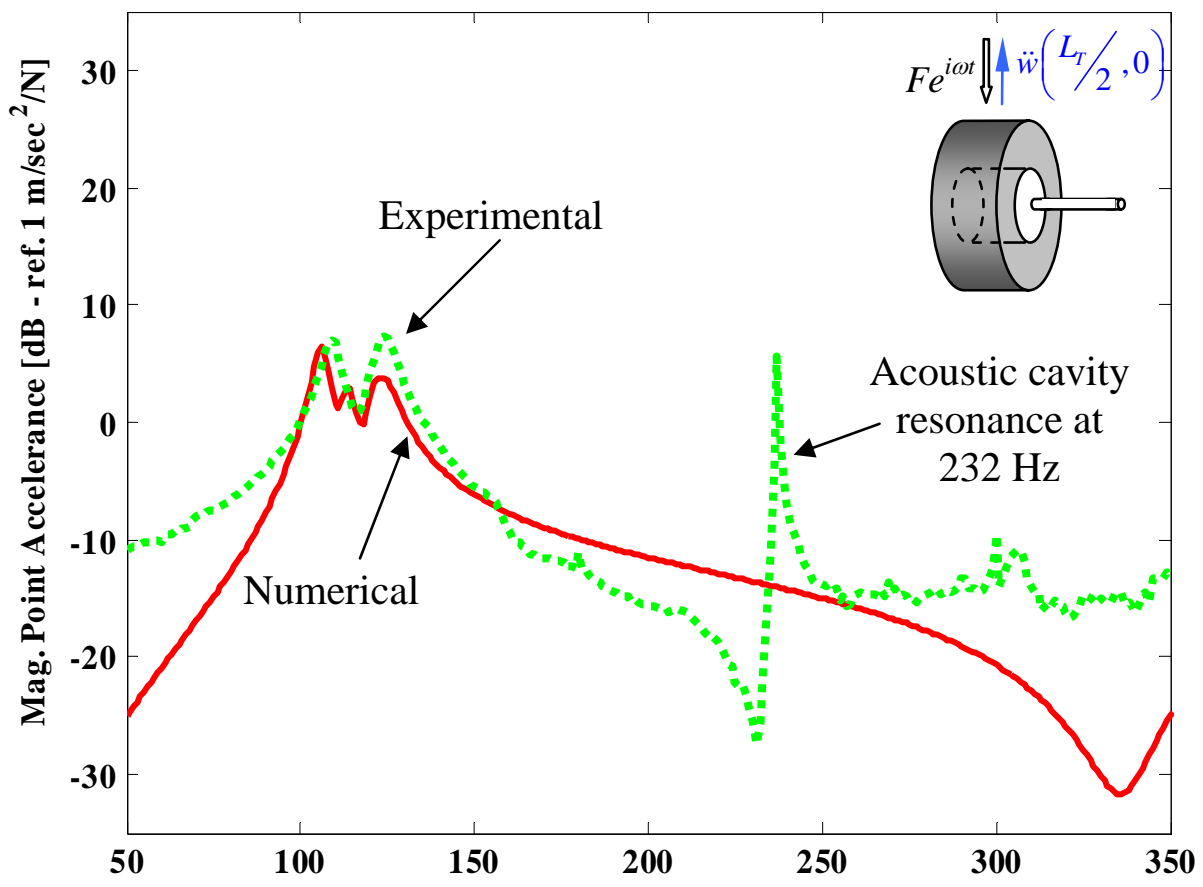

Figure 2.14: Point accelerance for the in vacuo free tire at the location of the external force. Acoustic cavity resonance not included in the model. 


\subsection{DEFORMED TIRE NUMERICAL SIMULATIONS}

The deformed tire dynamics is also simulated for a contact patch depth $\bar{h}=0.01 \mathrm{~m}$ and a harmonic oscillating $\varepsilon=\bar{h} \times 10^{-3} \mathrm{~m}=1 \times 10^{-5} \mathrm{~m}$. The simulation was performed using 124 modes corresponding to $m=1, . ., 4$ and $n=0, . ., 30$. The modal amplitude response for the fundamental mode is shown in Figure 2.15. This figure shows a very different response above the mode resonance as compared to the un-deformed or free tire model in Figure 2.10. The response shows a drop around $181 \mathrm{~Hz}$ which is due to the vanishing of the modal force given by the right side of equation (2.48). Note that in this case the modal force is frequency dependent.

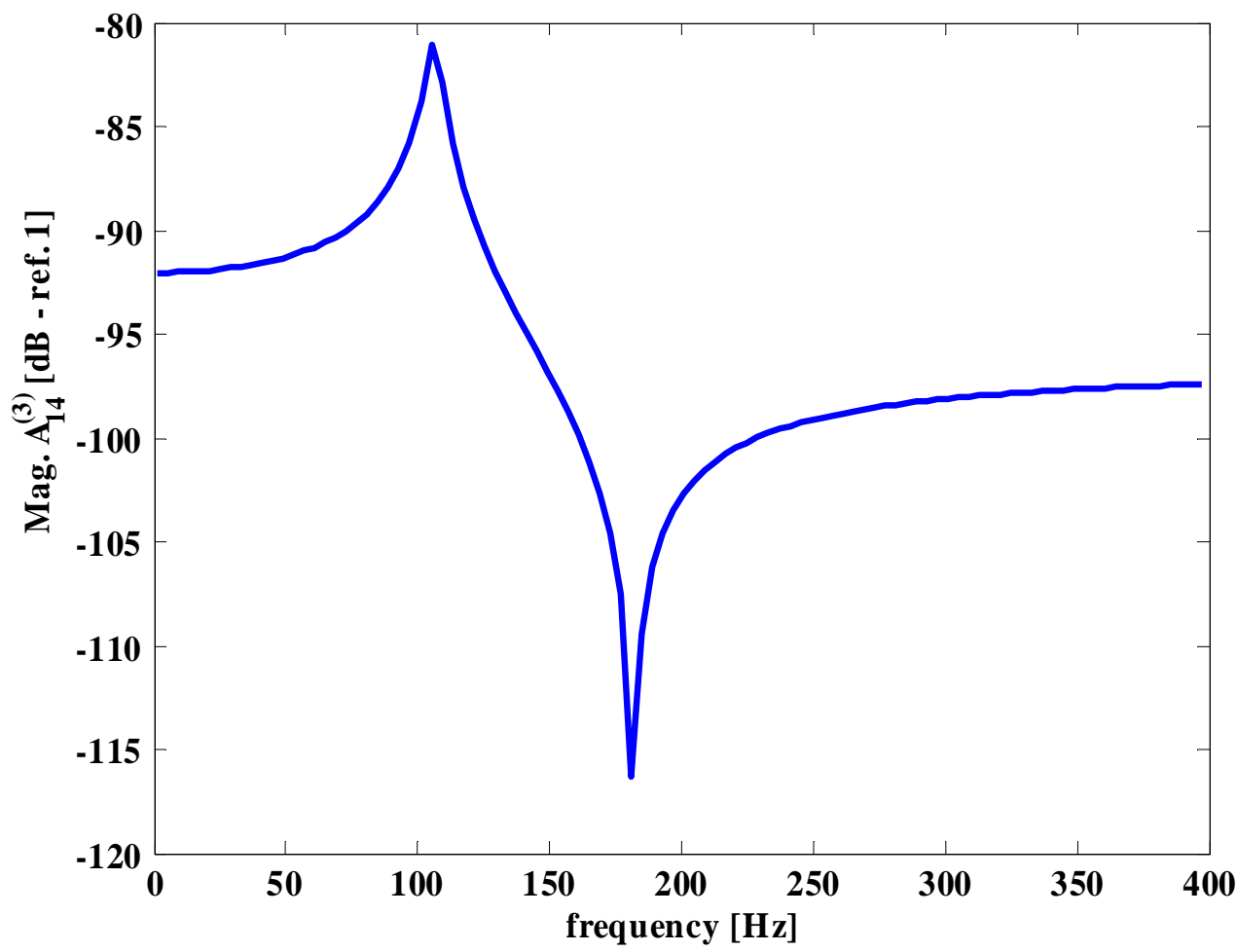

Figure 2.15: Structural Modal Amplitude for mode $\Phi_{14}^{(1)}$ with resonance at $105.9 \mathrm{~Hz}$ for the deformed tire model. 
The vertical force transmitted to the spindle for the deformed tire model is show in Figure 2.16. The radial $(1,1)$ and tangential $(1,1)$ modes are again the only modes contributing to the vertical force. Comparing to the case of the "free tire" in Figure 2.11, the contact patch input yields a different response where both modes are now equally important.

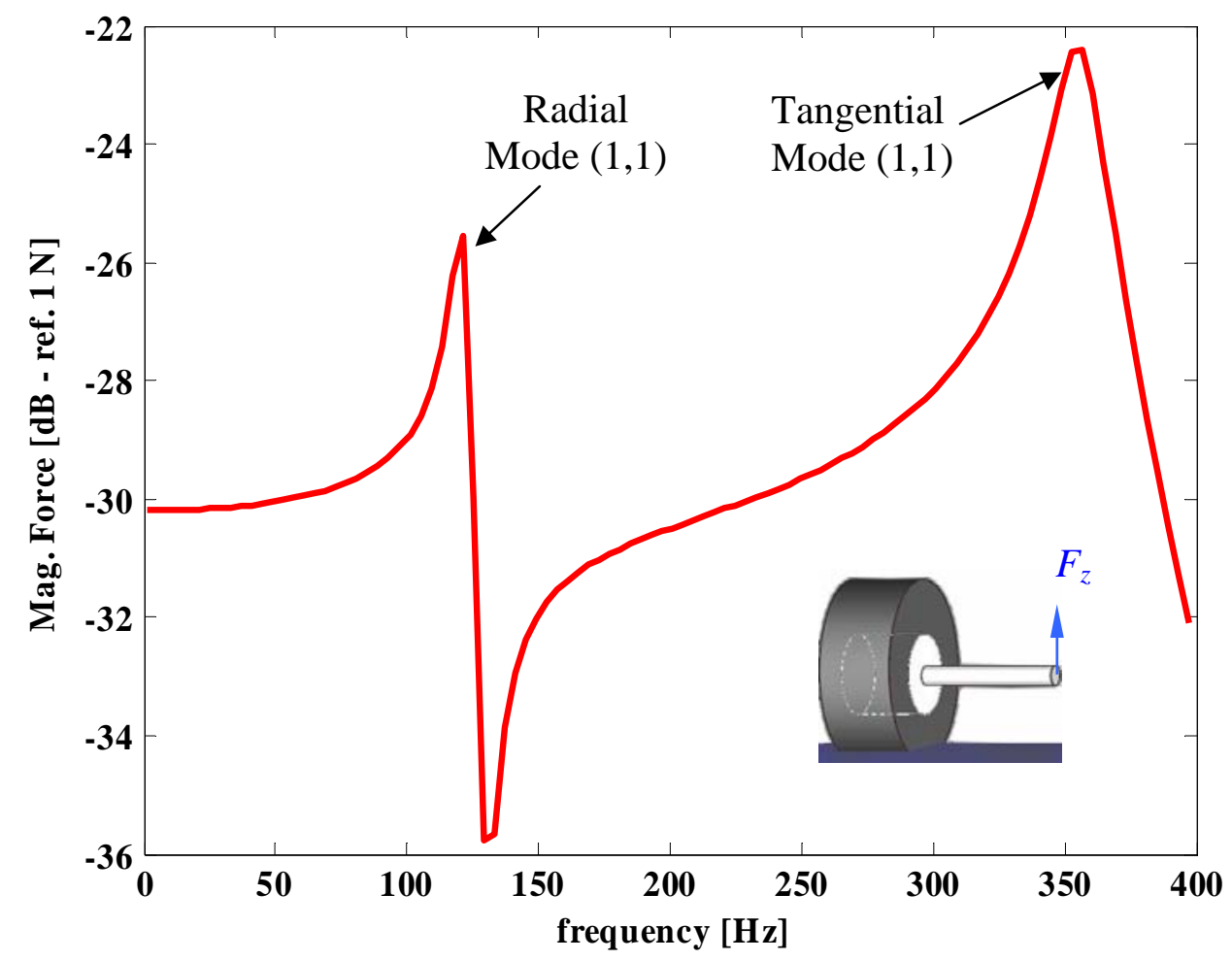

Figure 2.16: Vertical Forces $F_{z}$ at the spindle.

The horizontal moment at the spindle is shown in Figure 2.17. The radial $(1,1)$ and tangential $(1,1)$ mode are again the only ones present in the horizontal moment. Comparison with the horizontal moment obtained in the "free tire" model shows that the contacts patch excitation gives a response where both modes are equally dominating in the frequency range [0-400] Hz. 


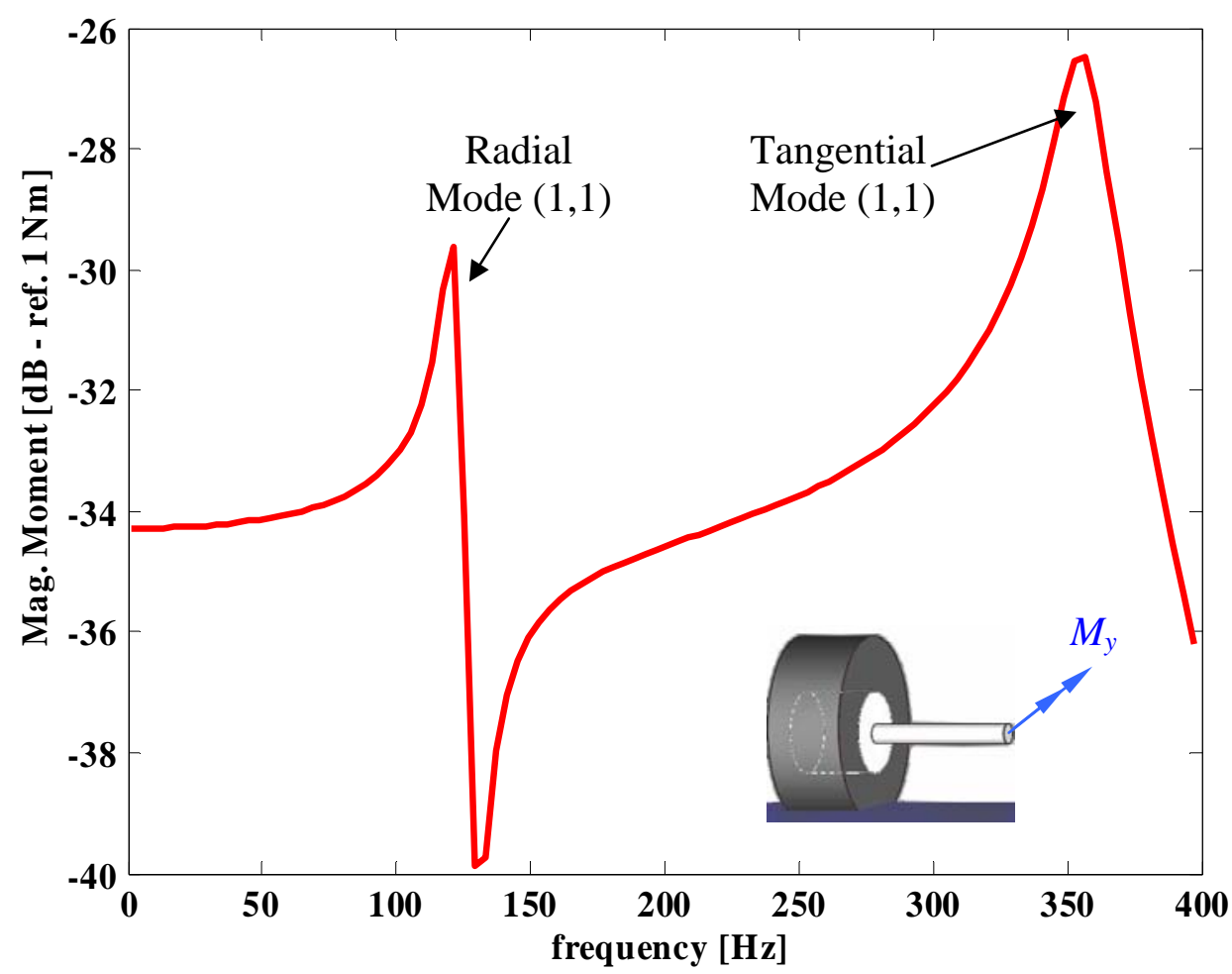

Figure 2.17: Horizontal Moment $\mathrm{M}_{\mathrm{y}}$ at the spindle.

Figure 2.18 shows the radial displacement for the location $(x=L / 2, \theta=0)$ on the tire. The response obtained for the "deformed tire" case is very different form the one obtained in the "free tire" case. Figure 2.18 shows that many modes are present in the response of the "deformed tire”. The distributed nature of the contact patch excites most of the modes. This distributed nature is given by the corresponding components of the contact patch expansion. 


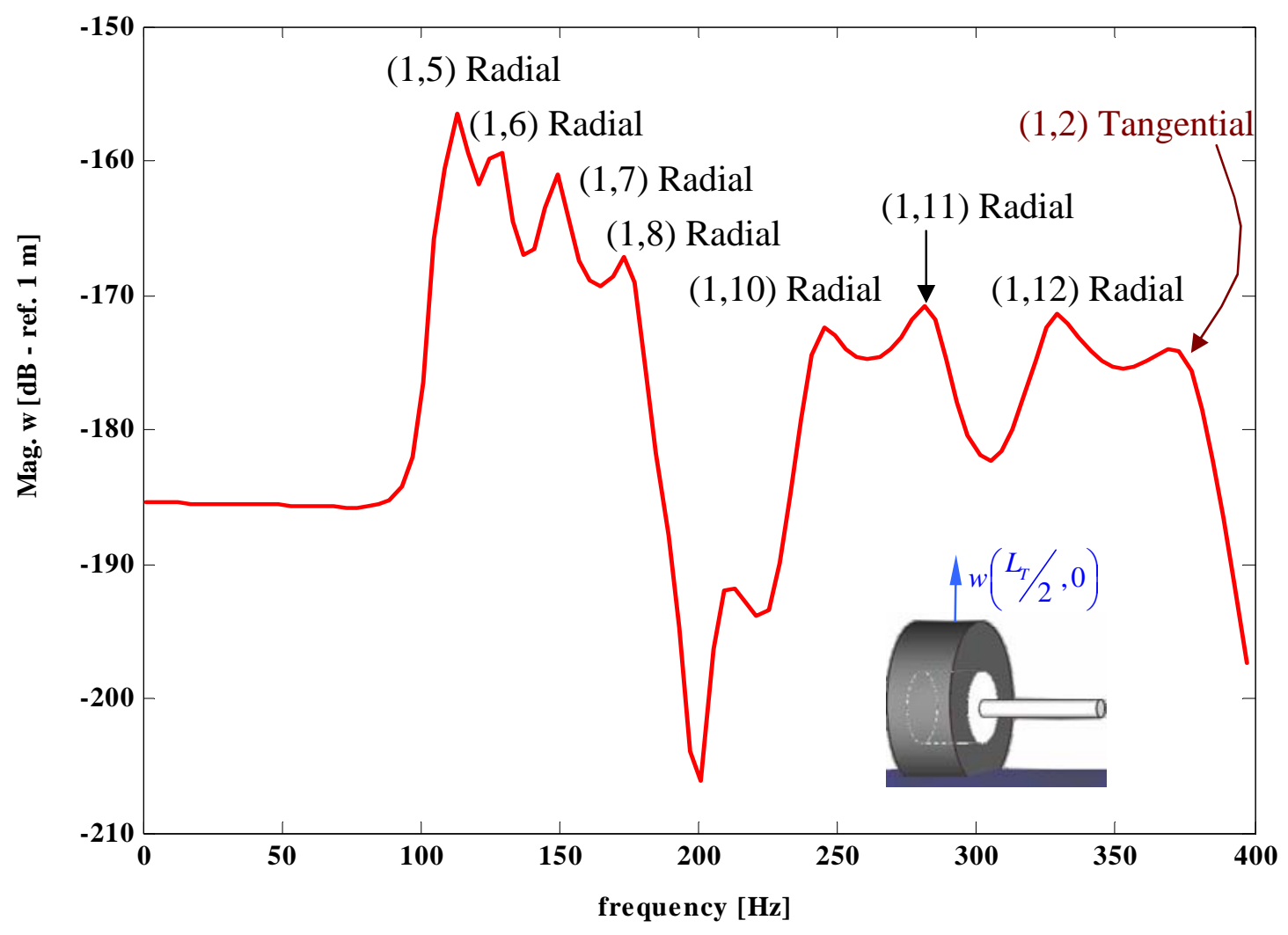

Figure 2.18: Radial displacement for the in vacuo deformed tire model at

$$
\text { location }\left(x=\frac{L_{T}}{2}, \theta=0\right) \text {. }
$$

Figure 2.19 shows a comparison between the point accelerance calculated by the deformed tire model (without the acoustic cavity) and measured in an experimental test by Yamauchi and Akiyoshi (2002). From Figure 2.19, the tire model was able to capture relatively well the general dynamic behavior of the experiments. There are two main differences between the numerical and experimental results. Since the modeling of the acoustic cavity is not yet included, the numerical results do not capture the acoustic resonance at around $230 \mathrm{~Hz}$. The other difference occur at low frequencies $(<100 \mathrm{~Hz})$ where the experimental data shows a resonance like behavior not shown by the model. It is speculated that the resonance at around $35 \mathrm{~Hz}$ doesn't belong to the tire but is most likely associated to the experimental setup mounting and thus not modeled. Note that 
from Table 2.2 the fundamental tire frequency is at $105 \mathrm{~Hz}$. In general, the model predicts well the dynamic behavior of the deformed tire thus validating the modeling approach.

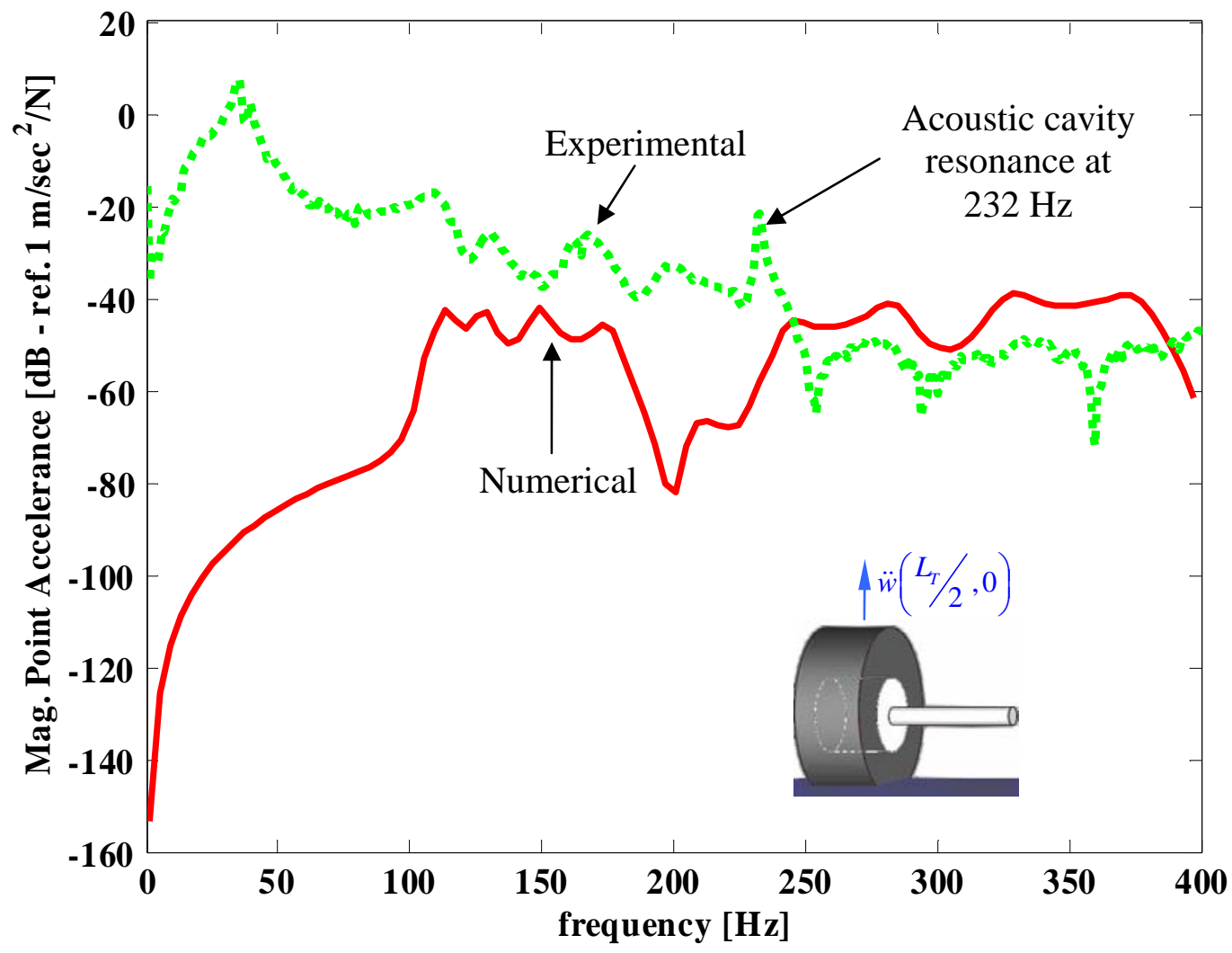

Figure 2.19: Point Accelerance for the in vacuo deformed tire model at location $\left(x=\frac{L_{T}}{2}, \theta=0\right)$. 


\title{
CHAPTER 3: COUPLED TIRE MODEL
}

\author{
he tire models developed in Chapter 2 do not include the effect of the tire \\ acoustic cavity. To account for the tire acoustic cavity in the structural \\ response, an expression for the acoustic pressure produced by the motion of \\ L the tire has to be obtained. To this end, the acoustic mode shapes and
} natural frequencies of this cavity are computed (Fahy, 2000). The eigenproperties are then used to solve for the Green's functions, which allow to compute the acoustic pressure due to the tire motion. In this Chapter, the modeling of the tire acoustic cavity is included in the structural models developed in Chapter 2. The "free tire" and "deformed tire" models are evaluated numerically and validated by comparison to experimental results.

\subsection{THE INTERIOR ACOUSTIC PROBLEM}

The problem here is to obtain an expression for the acoustic pressure $p(\underset{\sim}{r})$ induced by the tire motion. The acoustic cavity pressure in turn affects the tire response i.e. experimental feedback. In Figure 3.1, the problem of finding the acoustic pressure in an enclosure is presented.

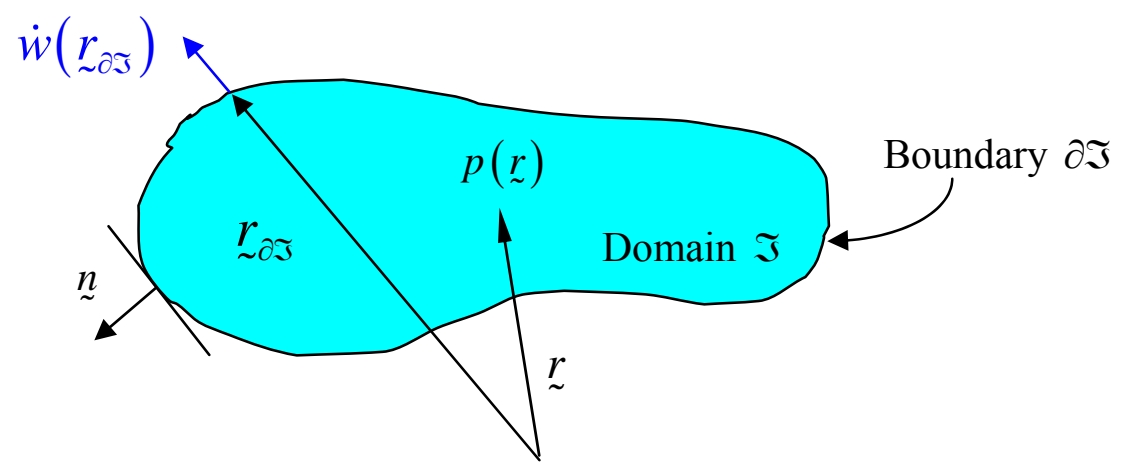

Figure 3.1: Interior acoustic problem. 
The acoustic cavity is the volume $\mathfrak{I}$ defined by the elastic boundary $\partial \mathfrak{I}$. The motion of the boundary is given by the velocity $\dot{W}(\underset{\sim}{r} \underset{\mathfrak{I}}{ })$ in the normal direction $\underset{\sim}{n}$ to the surface creates the acoustic pressure $p(\underset{\sim}{r})$

Before addressing the general problem of finding $p \underset{\sim}{r})$, a discussion on the general boundary conditions for this type of problem is presented. The boundary conditions on the domain surface $\partial \mathfrak{I}$, i.e. inside cavity surface, can be expressed as

$$
\alpha p(\underset{\sim}{r})+\left.\beta \frac{\partial p(\underset{\sim}{r})}{\partial n}\right|_{\partial \mathfrak{I}}=B(\underset{\sim}{r}), \quad \text { on } \partial \mathfrak{I}
$$

where $n$ is the normal to the boundary $\partial \mathfrak{I}, p \underset{\sim}{r})$ the acoustic pressure and $\alpha, \beta$, and $B$ are given functions evaluated on the region $\partial \mathfrak{I}$. The boundary condition relates the values of $p(\underset{\sim}{r})$ on $\partial \mathfrak{I}$ and the flux of $p(\underset{\sim}{r})$ through $\partial \mathfrak{I}$. The condition of $\alpha \geq 0, \beta \geq 0$, and $\alpha+\beta>0$ on $\partial \mathfrak{I}$ are required (Zauderer, 1989). If $\alpha \neq 0$ and $\beta=0$, the boundary condition is called of the first kind or Dirichlet ${ }^{1}$ condition. If $\alpha=0$ and $\beta \neq 0$, the boundary condition is called of the second kind or Neumann ${ }^{2}$ condition. If

\footnotetext{
${ }^{1}$ Dirichlet, Johan Peter Gastav Lejeune (1805-1859)
}

Dirichlet was a German mathematician who studied at a Jesuit college and then at the Collège de France. He became a tutor for the royalty of France and met many famous mathematicians. Although Dirichlet's main interest was in number theory, he also contributed in calculus and physics. Dirichlet returned to Germany in 1826 and taught at the military academy and the University of Berlin. He was good friends with colleague Carl Jacobi. Dirichlet investigated the solution and equilibrium of systems of differential equations and discovered many results on the convergence of series. In 1855, Dirichlet succeeded Gauss as the professor of mathematics at Göttingen.

(Source:http://occawlonline.pearsoned.com/bookbind/pubbooks/thomas awl/chapter1/medialib/custom3/bi os/dirichlet.htm )

\section{John von Neumann}

(1903-1957)

Johnny, as it seems everyone called him, was one of those people who are so bright it's hard to believe they were human. (Maybe he wasn't. There's an old joke about the Fermi Paradox, a problem which occurred to Enrico Fermi one day at Los Alamos: where are They? If there are intelligent aliens out there in the universe, why aren't they here yet? A million years is nothing, as the universe reckons things, but, judging 
$\alpha \neq 0$ and $\beta \neq 0$, the boundary condition is called of the third kind or mixed kind. When $B$ does not vanish, the boundary conditions are of inhomogeneous type. If $B$ and $\alpha$ vanish at the boundary the condition is "hard wall".

Consider now a general differential operator $L$ and

$$
\operatorname{Lp}(\underset{\sim}{r})=F(\underset{\sim}{r}) \text { in } \mathfrak{I}
$$

where $F(\underset{\sim}{r})$ represent the external influences on the system. The operator $L$ is not assumed to be formally self-adjoint, $L \neq \tilde{L}$ in order to keep the richness in the development. Therefore, the adjoint operator $\tilde{L}$ of $L$ satisfies the equation (Roach, 1970)

$$
\tilde{L} G\left(\left.\underset{\sim}{r}\right|_{\sim}\right)=\delta\left(\underset{\sim}{r}-{\underset{\sim}{\infty}}_{0}\right)
$$

where $G\left(\left.\underset{\sim}{r}\right|_{\sim}\right)$ is the Green's function. The boundary condition are given by the adjoint of the operator $B(\underset{\sim}{r})$.

The inner product upon the domain $\mathfrak{I}$ is defined as

$$
\langle x, y\rangle=\int_{\mathfrak{I}} x \bar{y} d \mathfrak{I}
$$

from our own track-record, a species only that much older than us would have technology which would blow our minds, pretty close to limits set by physical laws. Leo Szilard is supposed to have answered Fermi: "Maybe they're already here, and you just call them Hungarians.") About the only large current of the natural sciences in this century which von Neumann's work has not added to is molecular biology. Almost everything else of any significance he touched: mathematical logic; pure math; quantum physics; computing (which, as we know it, is largely his invention), cybernetics and automata theory; the Bomb; turbulence; game theory (another invention) and so economics, evolutionary biology, and the theory of war and conflict; artificial life, cellular automata (a third invention), the theory of self-reproduction (which, with molecular biology, finally killed off any last lingering hopes for vitalism) and artificial evolution. What many of us like to think of as new and profound changes in the way science works, brought about by computer modeling and simulation, were foreseen and called for by von Neumman in the '40s. If any one person can be said to be the intellectual ancestor of complexity and all that travels alongside it, it was Johnny. His only real rival for the honor is Norbert Wiener, a better man but a less overwhelming scientist.

( Source: $\underline{\text { http://cscs.umich.edu/ crshalizi/notebooks/von-neumann.html ) }}$ 
where the over-bar in equation (3.4) denotes complex conjugates (Naylor and Sell, 2000).

Taking the inner product on equation (3.2) with the Green's function $G\left(\left.\underset{\sim}{r}\right|_{\sim}\right)$, it gives

$$
\left\langle G\left(\underset{\sim}{r} \mid r_{\sim}\right), L p(\underset{\sim}{r})\right\rangle=\left\langle G\left(\underset{\sim}{r} \mid r_{\sim}\right), F(\underset{\sim}{r})\right\rangle
$$

and again the inner product on equation $(3.3)$ by $p(\underset{\sim}{r})$, it yields

$$
\left\langle p(\underset{\sim}{r}), \tilde{L} G\left(\underset{\sim}{r} \mid r_{\sim}\right)\right\rangle=\langle p(\underset{\sim}{r}), \delta(\underset{\sim}{r}-\underset{\sim}{r})\rangle
$$

Subtracting equation (3.6) from(3.5), the following relation holds

$$
\left\langle G\left(\underset{\sim}{r} \mid \sim_{\sim}\right), L p(\underset{\sim}{r})\right\rangle-\langle p(\underset{\sim}{r}), \tilde{L} G(\underset{\sim}{r} \mid \underset{\sim}{r})\rangle=\left\langle G\left(\left.\underset{\sim}{r}\right|_{\sim} ^{r}\right), F(\underset{\sim}{r})\right\rangle-\langle p(\underset{\sim}{r}), \delta(\underset{\sim}{r}-\underset{\sim}{r})\rangle
$$

or

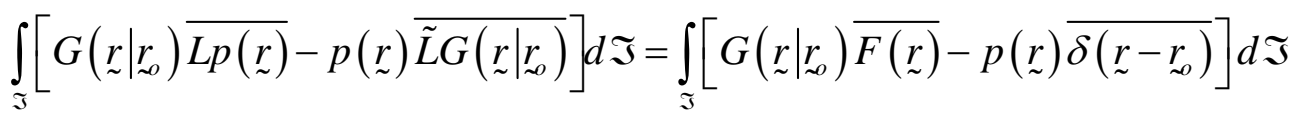

Using the following properties of the three-dimensional Dirac ${ }^{3}$ delta

\footnotetext{
${ }^{3}$ Paul Adrien Maurice Dirac

(August 81902 - October 20 1984)
}

Dirac was born in Bristol, Gloucestershire, England. In 1926 he developed a version of quantum mechanics, which included "Matrix Mechanics" and "Wave Mechanics" as special cases.

In 1928, building on Pauli's work on nonrelativistic spin systems, he derived the Dirac equation, a relativistic equation describing the electron. This allowed Dirac to formulate the Dirac sea and predict the existence of the positron, the electron's anti-particle; the positron was subsequently observed by Anderson in 1932. Dirac explained the origin of quantum spin as a relativistic phenomenon.

Dirac's Principles of Quantum Mechanics, published in 1930, pioneered the use of linear operators as a generalization of the theories of Heisenberg and Schrödinger. It also introduced the bracket notation, in which $|\psi\rangle$ denotes a state vector in the Hilbert space of a system and $\langle\psi|$ its dual vector. Dirac also introduced Dirac's delta function. 


$$
\int_{\mathfrak{I}} p(\underset{\sim}{r}) \overline{\delta\left(\underset{\sim}{r}-r_{\sim}\right)} d \mathfrak{I}=\left\{\begin{array}{lr}
p\left(r_{\sim}\right), & \underset{\sim}{r} \text { within } \mathfrak{I} \\
\frac{1}{2} p\left(r_{\sim}\right), & \underset{\sim}{r} \text { on } \partial \mathfrak{I} \\
0, & \underline{r}_{\sim} \text { outside of } \mathfrak{I}
\end{array}\right.
$$

the expression for the acoustic pressure is given by

$$
p(\underset{\sim}{\underset{\sim}{\alpha})})=\varepsilon_{\mathfrak{I}} \int_{\mathfrak{J}}\left[G\left(\left.\underset{\sim}{r}\right|_{\sim}\right) \overline{F(\underset{\sim}{r})}+p(\underset{\sim}{r}) \overline{\tilde{L} G\left(\left.\underset{\sim}{r}\right|_{\sim} ^{r}\right)}-G\left(\left.\underset{\sim}{r}\right|_{\sim}\right) \overline{L p(\underset{\sim}{r})}\right] d \mathfrak{I}
$$

where

$$
\varepsilon_{\mathfrak{I}}=\left\{\begin{array}{lr}
1, & \underset{\sim}{r} \text { within } \mathfrak{I} \\
2, & {\underset{\sim}{\sim}}_{0} \text { on } \partial \mathfrak{I}
\end{array}\right.
$$

Equation (3.10) becomes

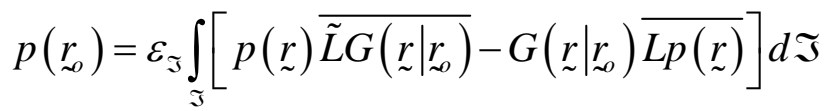

The integrand of equation (3.12) is a divergence expression where $\tilde{L}$ is the formally adjoint operator of $L$. Moreover if $\tilde{L}=L$, the operator $L$ is formally self-adjoint. The boundary condition associated to the given equation and its formal adjoint boundary condition play an important roll to determine whether or not the problem is self-adjoint. Therefore, it is possible for an operator to be formally self adjoint but for the problem

Dirac shared the Nobel Prize for physics in 1933 with Erwin Schrödinger "for the discovery of new productive forms of atomic theory". Dirac was Lucasian professor of mathematics at Cambridge from 1932 to 1969 and an undergraduate at Bristol University. The Dirac Prize is awarded in his honor.

Dirac died in Tallahassee, Florida.

(Source: http://www.brainyencyclopedia.com/encyclopedia/p/pa/paul_dirac.html) 
associated with this operator and its adjoint to be nonself-adjoint. But if the operator is not self-adjoint the problem cannot be self adjoint.

Equation (3.12) is a general expression for the acoustic pressure. Further development requires specifying the character of the operator $L$ used to model the acoustic problem.

\subsection{THE TIRE ACOUSTIC CAVITY}

The operator for this particular application is given by the linear wave equation, which is self-adjoint $(\tilde{L}=L)$. That is

$$
\nabla^{2} p-\frac{1}{c^{2}} \frac{d^{2} p}{d t^{2}}=0
$$

with boundary condition

$$
\left.\frac{\partial p(\underset{\sim}{r})}{\partial n}\right|_{\partial \mathfrak{I}}=-\rho \frac{\partial \dot{w}(\underset{\sim}{r})}{\partial t} \quad \text { with } \underset{\sim}{r} \in \partial \mathfrak{I}
$$

where $\dot{w}(\underset{\sim}{r})$ is the velocity at the boundary and $p$ is assumed to have the form

$$
p(r, \theta, x, t)=p(r, \theta, x) e^{i \omega t}
$$

Since the boundary conditions is harmonic, replacing equation (3.15) into (3.13) and (3.14), the resultant equations is given by

$$
\nabla^{2} p+k^{2} p=0 \quad \text { and }\left.\frac{\partial p(\underset{\sim}{r})}{\partial n}\right|_{\partial \mathfrak{T}}=-i \omega \rho \dot{w}(\underset{\sim}{r}) \quad \text { with } \underset{\sim}{\underset{\sim}{r} \in \partial \mathfrak{I}}
$$


this equation is the so called Helmholtz ${ }^{4}$ equation, in where $k$ is the free field acoustic wavenumber. Then, using equation (3.12) the acoustic pressure becomes

$$
\begin{aligned}
p(\underset{\sim}{r})= & \varepsilon_{\mathfrak{I}} \int_{\mathfrak{I}}\left\{p(\underset{\sim}{r})\left[\nabla^{2} G\left(\left.\underset{\sim}{\underset{\sim}{r}}\right|_{\sim}\right)+k^{2} G\left(\underset{\sim}{r}||_{\sim}\right)\right]-G\left(\left.\underset{\sim}{\underset{\sim}{r}}\right|_{\sim}\right)\left[\nabla^{2} p(\underset{\sim}{r})+k^{2} p(\underset{\sim}{r})\right]\right\} d \mathfrak{I} \\
& \text { with } \underset{\sim}{r} \in \partial \mathfrak{I}
\end{aligned}
$$

or

$$
\begin{aligned}
p(\underset{\sim}{r})= & \varepsilon_{\Im} \int_{\mathfrak{I}}\left\{\left[p(\underset{\sim}{r}) \nabla^{2} G\left(\left.\underset{\sim}{r}\right|_{\sim}\right)-G\left(\left.\underset{\sim}{r}\right|_{\sim}\right) \nabla^{2} p(\underset{\sim}{r})\right]+\left[p(\underset{\sim}{r}) k^{2} G\left(\left.\underset{\sim}{r}\right|_{\sim}\right)-G\left(\left.\underset{\sim}{r}\right|_{\sim}\right) k^{2} p(\underset{\sim}{r})\right]\right\} d \mathfrak{I} \\
& \text { with } \underset{\sim}{r} \in \partial \mathfrak{I}
\end{aligned}
$$

The second term in the integral of equation (3.18) vanishes. The simplification in the inner product given in the integration is due to the self-adjoint property of the Helmholtz operator. Therefore, the operator left in equation (3.18) is the Laplace's ${ }^{5}$ operator, which also is self adjoint (Kreyszig, 1978). Then, it is possible to write

\footnotetext{
${ }^{4}$ Helmholtz, Hermann von (1821-1894)
}

German physicist who first extended Joule's results to a general principle. He expressed the relationship between mechanics, heat, light, electricity, and magnetism by treating them all as manifestations of a single force. He presented a mathematical proof for his Law of Conservation of Force in Über die Erhaltung der Kraft (On the Conservation of Force, 1847). Helmholtz's use of the word "force" corresponds to what later became known as energy, so his law actually amounted to a statement of the Law of conservation of energy.

Helmholtz sought to synthesize Maxwell's electromagnetic theory of light with the central force theorem. To accomplish this, he formulated an electrodynamic theory of action at a distance in which electric and magnetic forces were propagated instantaneously. He attempted to demonstrate that body heat and muscular action of animals could be produced by the oxidation of foodstuffs.

(Source: http://scienceworld.wolfram.com/biography/Helmholtz.html )

\section{${ }^{5}$ Pierre-Simon Laplace}

(March 23, 1749 - March 5, 1827) was a French mathematician, the discoverer of the Laplace transform and Laplace's equation. He was a believer in causal determinism.

One curious formula for the probability that the sun would rise was given by Laplace. He claimed that this probability was $(d+1) /(d+2)$-, where $d$ is the number of days that the sun has risen in the past. Laplace claimed that this formula, known as (Laplace) Rule of Succession, applied in all cases where we knew nothing, or where what we did know was swamped by what we didn't. It is still used as an estimator for the probability of an event if we know the event space, but only have a small number of samples.

Laplace strongly believed in causal determinism, which is expressed in the following citation:

"We may regard the present state of the universe as the effect of its past and the cause of its future. An intellect which at any given moment knew all of the forces that animate nature and the mutual positions of the beings that compose it, if this intellect were vast enough to submit the data to analysis, could condense 


$$
\begin{aligned}
p(\underset{\sim}{r}) & =\varepsilon_{\mathfrak{I}} \int_{\mathfrak{I}}\left[p(\underset{\sim}{r}) \nabla^{2} G\left(\left.\underset{\sim}{r}\right|_{\sim}\right)-G\left(\left.\underset{\sim}{r}\right|_{\sim} ^{r}\right) \nabla^{2} p(\underset{\sim}{r})\right] d \mathfrak{I} \\
& =\varepsilon_{\mathfrak{I}} \int_{\mathfrak{I}} \nabla \cdot\left[p(\underset{\sim}{r}) \nabla G\left(\left.\underset{\sim}{r}\right|_{\sim}\right)-G\left(\left.\underset{\sim}{\underset{\sim}{r}}\right|_{\sim}\right) \nabla p(\underset{\sim}{r})\right] d \mathfrak{I} \\
& \text { with } \underset{\sim}{r} \in \partial \mathfrak{I}
\end{aligned}
$$

Applying Gauss's second identity on (3.19) (Griffel, 1985), it gives

$$
\begin{aligned}
& p(\underset{\sim}{r})=\varepsilon_{\mathfrak{I}} \int_{\mathfrak{I}} \nabla \cdot\left[p(\underset{\sim}{r}) \nabla G(\underset{\sim}{\underset{\sim}{r}}{\underset{\sim}{r})})-G\left(\left.\underset{\sim}{\underset{\sim}{r}}\right|_{\sim}\right) \nabla p(\underset{\sim}{r})\right] d \mathfrak{I} \\
& =\varepsilon_{\Im} \int_{\partial \mathfrak{I}} \underset{\sim}{n} \bullet\left[p(\underset{\sim}{r}) \nabla G\left(\left.\underset{\sim}{r}\right|_{\sim}\right)-G\left(\left.\underset{\sim}{r}\right|_{\sim}\right) \nabla p(\underset{\sim}{r})\right] d(\partial \mathfrak{I}) \\
& =\varepsilon_{\mathfrak{I}} \int_{\partial \mathfrak{I}}\left[p(\underset{\sim}{r}) \frac{\partial G\left(\left.\underset{\sim}{r}\right|_{\sim} ^{r_{0}}\right)}{\partial n}-G\left(\left.\underset{\sim}{r}\right|_{\sim} ^{r_{0}}\right) \frac{\partial p(\underset{\sim}{r})}{\partial n}\right] d(\partial \mathfrak{I}) \\
& \text { with } \underset{\sim}{r} \in \partial \mathfrak{I}
\end{aligned}
$$

A Green's function satisfying the "rigid wall" (Wu, 2000) condition implies that

$$
\frac{\partial G\left(\left.\underset{\sim}{r}\right|_{\sim}\right)}{\partial n}=0 \quad \text { on } \underset{\sim}{r} \in \partial \mathfrak{I}
$$

Replacing the boundary condition given by equation (3.14) and the condition (3.21), the acoustic pressure produced by the motion of the boundary is given by

into a single formula the movement of the greatest bodies of the universe and that of the lightest atom; for such an intellect nothing could be uncertain and the future just like the past would be present before its eyes."

This intellect is often referred to as Laplace's demon (cf. Maxwell's demon). The discoveries of modern physics, especially quantum physics and uncertainty principle proved that the existence of such an intellect is not possible even in principle.

(Source: http://en.wikipedia.org/wiki/Laplace ) 


$$
\begin{aligned}
& p\left(r_{\sim}\right)=\varepsilon_{\mathfrak{I}} \int_{\partial \mathfrak{I}} i \omega \rho \dot{w}(\underset{\sim}{r}) G\left(\left.\underset{\sim}{r}\right|_{\sim}\right) d(\partial \mathfrak{I}) \\
& \text { with } \underset{\sim}{r} \in \partial \mathfrak{I}
\end{aligned}
$$

To find the acoustic pressure and thus the dynamics of the tire acoustic the hard wall Green's function are needed. To this end, the eigenvalue problem associated to the operator for the hard wall boundary condition need to be solved. The eigenproperties are then used to construct the Green's functions. Then, the Green's function is used to compute the pressure inside the tire acoustic cavity due to the motion of the flexible shell using (3.22). The tire acoustic cavity is modeled as a cylindrical cavity with annular section as shown in Figure 3.2.

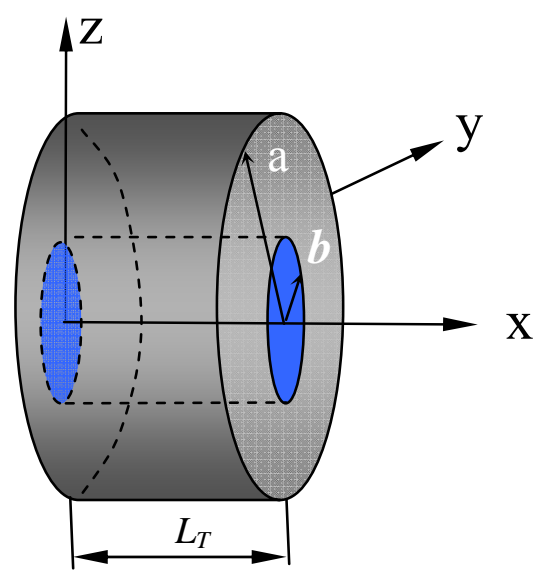

Figure 3.2: Finite cylindrical cavity.

Due to the geometry of the tire acoustic cavity, the Helmholtz equation is expressed in cylindrical coordinates as follow

$$
\frac{\partial^{2} p}{\partial r^{2}}+\frac{1}{r} \frac{p}{\partial r}+\frac{1}{r^{2}} \frac{\partial^{2} p}{\partial \theta^{2}}+\frac{\partial^{2} p}{\partial x^{2}}+k^{2} p=0
$$

The rigid wall boundary conditions are of the first kind or Dirichlet conditions defined as 


$$
\left(\frac{\partial p}{\partial x}\right)_{x=0}=\left(\frac{\partial p}{\partial x}\right)_{x=L_{T}}=\left(\frac{\partial p}{\partial r}\right)_{r=a}=\left(\frac{\partial p}{\partial r}\right)_{r=b}=0
$$

The acoustic pressure $p$ is assumed to be the solution of equation (3.23) in the form

$$
p(r, \theta, x, t)=p(r, \theta, x) e^{i \omega_{q p l} t}=\hat{A}_{q p l} \Psi_{q p l}(r, \theta, x) e^{i \omega_{q p l} t}
$$

where $\Psi_{q p l}(r, \theta, x)$ is the acoustic pressure mode shape corresponding to the natural angular frequency $\omega_{q p l}$ and $\hat{A}_{q p l}$ is the acoustic modal amplitude. The three indexes used in the acoustic pressure mode shapes have the following meaning:

- $q$ is the mode index on the axial direction.

- $\quad p$ index is the acoustic mode order on the azimuth direction, which also couples with the radial direction for the cylindrical cavity .

- $\quad l$ index represents the mode order in the radial direction.

The details of the procedure used to solve the problem can be seen in the Appendix C. Replacing equation (3.25) into (3.23), the solution of equation (3.23) under the boundary conditions (3.24) is

$$
\begin{gathered}
\Psi_{q p l}(r, \theta, x)=\left[Y_{p}^{\prime}\left(k_{p l} a\right) J_{p}\left(k_{p l} r\right)-J_{p}^{\prime}\left(k_{p l} a\right) Y_{p}\left(k_{p l} r\right)\right] \cos (p \theta) \cos \left(k_{x q} x\right) \\
\text { with } q, p, l=0,1,2, \ldots
\end{gathered}
$$

where $J_{p}$ and $Y_{p}$ are the Bessel's functions of First and Second Kind, respectively; $k_{p l}$ is the radial wavenumber which couples with the azimuth direction, and $k_{x q}$ is the wavenumber associated to the axial direction. The details of the eigensolution can be found in Appendix D. 
The eigen-wavenumber of the Helmholtz operator is given by

$$
k_{q p l}=\frac{\omega_{q p l}}{c}=\frac{2 \pi f_{q p l}}{c} \cong \sqrt{k_{x q}^{2}+k_{p l}^{2}}=\sqrt{\left(\frac{l \pi}{a-b}\right)^{2}+\left(\frac{2 p}{a+b}\right)^{2}+\left(\frac{q \pi}{L_{T}}\right)^{2}}
$$

The eigenfunction $\Psi_{q p l}(r, \theta, x)$ satisfies the following orthogonality property

$$
\int_{0}^{2 \pi} \int_{0}^{L_{T}} \int_{b}^{a} \Psi_{q p l}(x, \theta, r) \Psi_{r s o}(x, \theta, r) r d r d x d \theta=\Lambda_{q p l} \delta_{q r} \delta_{p s} \delta_{l o}
$$

where $\Lambda_{q p l}$ is the mode normalization factor.

Figure 3.3 shows the $\Psi_{010}$ acoustic mode shape. This acoustic mode shape is highlighted because it is the one that couples with the structure in the low frequency range of interest. Only this acoustic mode is analyzed in this dissertation because it is the culprit of the vertical excitation of the wheel. Moreover, the harmonic point force and the contact patch as inputs are capable only to excite this acoustic mode due to the symmetry of the problem.

The mode shape $\Psi_{010}$ has variation along the azimuth direction $\theta(\cos (p \theta))$, while the values in the radial direction are "almost constant", i.e. only a $2.5 \%$ of variation in the pressure from $r=a$ to $r=b$. Therefore, the radial sections can be assumed constant without much loss of generality. 

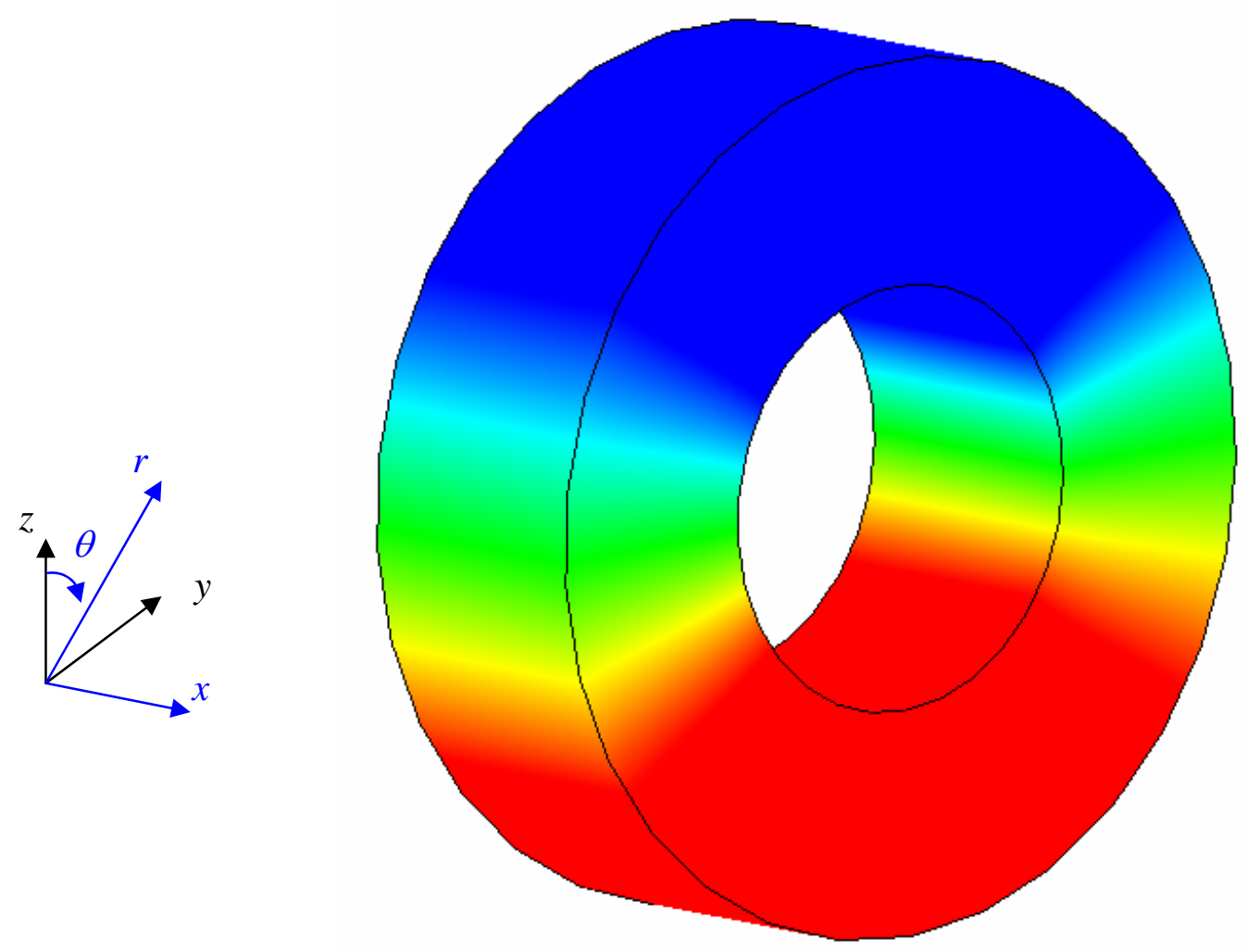

Figure 3.3: Acoustic Mode Shape $\Psi_{010}$ at $f_{010}=232 \mathrm{~Hz}$.

The next step is to use the spectral properties to find the Green's functions $G$ required in equation (3.22). The Green's function is the solution of

$$
\nabla^{2} G\left(x, \theta, r \mid x_{o}, \theta_{o}, r\right)_{o}+k^{2} G\left(x, \theta, r \mid x_{o}, \theta_{o}, r_{o}\right)=-\delta\left(x-x_{o}, \theta-\theta_{o}, r-r_{o}\right)
$$

with rigid boundary conditions. Note that the dimension of the Dirac delta $\delta\left(x-x_{o}, \theta-\theta_{o}, r-r_{o}\right)$ is $m^{-3}$.

The eigenfunctions $\Psi_{q p l}(x, \theta, r)$ satisfy the boundary condition of "rigid wall" given by equation (3.24). Therefore, these functions are candidates to expand the Green's function 
in the domain because the "rigid wall" condition is also required in $G$. Then, it is possible to write,

$$
G\left(x, \theta, r \mid x_{o}, \theta_{o}, r_{o}\right)=\sum_{q=0}^{Q} \sum_{p=0}^{P} \sum_{l=0}^{L} \tilde{B}_{q p l} \Psi_{q p l}(x, \theta, r)
$$

Replacing equation (3.30) into (3.29) yields (See Appendix C)

$$
-\sum_{q=0}^{Q} \sum_{p=0}^{P} \sum_{l=0}^{L} \tilde{B}_{q p l} k_{q p l}^{2} \Psi_{q p l}(r, \theta, x)+k^{2} \sum_{q=0}^{Q} \sum_{p=0}^{P} \sum_{l=0}^{L} \tilde{B}_{q p l} \Psi_{q p l}(x, \theta, r)=-\partial\left(x-x_{o}, \theta-\theta_{o}, r-r_{o}\right)
$$

The next step is to solve for $\tilde{B}_{q p l}$ by using the orthogonality condition of the acoustic modes shapes $\Psi_{q p l}$ as follow

$$
\begin{aligned}
& \int_{0}^{2 \pi} \int_{0}^{L_{r}} \int_{b}^{a} \Psi_{r s o}(x, \theta, r)\left[-\sum_{q=0}^{Q} \sum_{p=0}^{P} \sum_{l=0}^{L} \tilde{B}_{q p l} k_{q p l}^{2} \Psi_{q p l}(x, \theta, r)+k^{2} \sum_{q=0}^{Q} \sum_{p=0}^{P} \sum_{l=0}^{L} \tilde{B}_{q p l} \Psi_{q p l}(x, \theta, r)\right] r d r d x d \theta= \\
& =-\int_{0}^{2 \pi} \int_{0}^{L_{r}} \int_{b}^{a} \Psi_{r s o}(x, \theta, r) \partial\left(x-x_{o}, \theta-\theta_{o}, r-r_{o}\right) r d r d x d \theta
\end{aligned}
$$

which results in the following expression

$$
\tilde{B}_{q p l} \Lambda_{q p l}\left(k^{2}-k_{q p l}^{2}\right)=-\Psi_{q p l}\left(x_{o}, \theta_{o}, r_{o}\right) \frac{1}{\varepsilon_{\mathfrak{I}}}
$$

and

$$
\tilde{B}_{q p l}=\frac{\Psi_{q p l}\left(x_{o}, \theta_{o}, r_{o}\right)}{\Lambda_{q p l}\left(k_{q p l}^{2}-k^{2}\right)} \frac{1}{\varepsilon_{\mathfrak{I}}}
$$


Therefore, the Green's function given by equation (3.30) becomes

$$
G\left(x, \theta, r \mid x_{o}, \theta_{o}, r_{o}\right)=\sum_{q=0}^{Q} \sum_{p=0}^{P} \sum_{l=0}^{L} \frac{\Psi_{q p l}\left(x_{o}, \theta_{o}, r_{o}\right) \Psi_{q p l}(x, \theta, r)}{\Lambda_{q p l}\left(k_{q p l}^{2}-k^{2}\right)} \frac{1}{\varepsilon_{\mathfrak{I}}}
$$

This Green's function is symmetric or reciprocal, i.e. $G\left(x, \theta, r \mid x_{o}, \theta_{o}, r_{o}\right)=G\left(x_{o}, \theta_{o}, r_{o} \mid x, \theta, r\right)$ due to the self-adjoint character of the Helmholtz operator and the "rigid wall" boundary condition. Equation (3.35) is used into (3.22) to obtain the acoustic pressure due to the boundary motion, $\underset{w}{\dot{r}} \underset{\sim}{r})$ with $\underset{\sim}{r} \in \partial \mathfrak{I}$.

\subsection{COUPLED STRUCTURAL-ACOUSTIC PROBLEM}

The cavity-structure interaction problem is now solved. Figure 3.4 illustrates the problem of a structure and the interior acoustic pressure. It is important to remark that the description of the cavity-structural problem is quite complex due to the six indices required to describe the structural and acoustic modes. Here a brief description of the coupled problem will be presented for a general understanding of the solution approach.

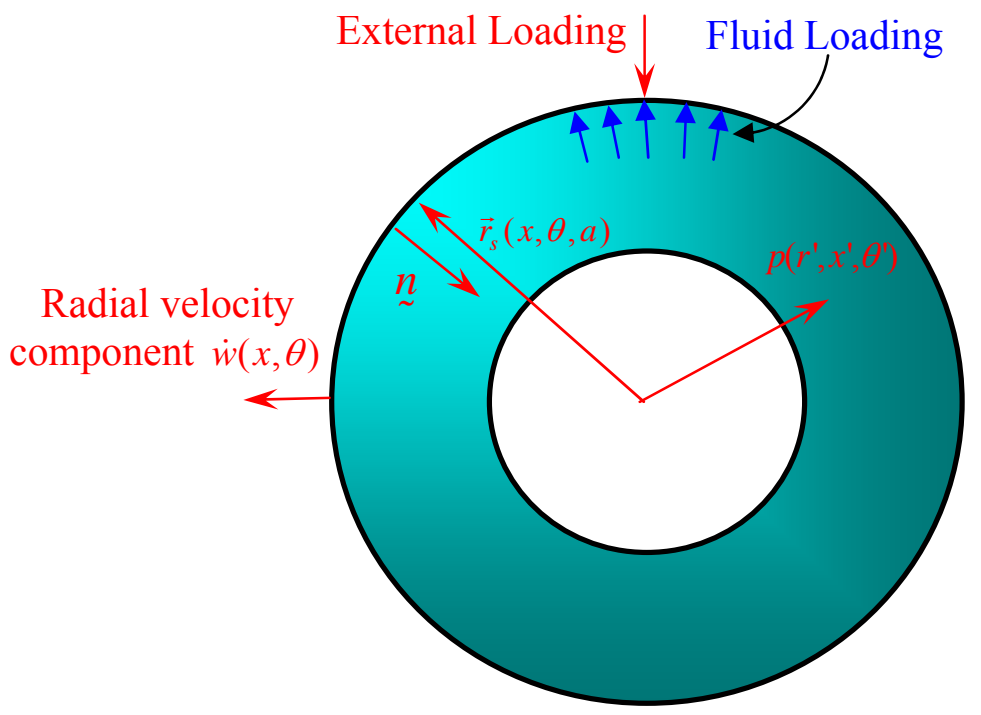

Figure 3.4: Structure radiating into an enclosed volume. 
The approach to solve the coupled problem is to include in the equation of motion of the tire the force due to the interior pressure i.e. fluid loading. However, the fluid forces depend on the velocity response of the structure which leads to the fluid feedback problem depicted in Figure 3.5.

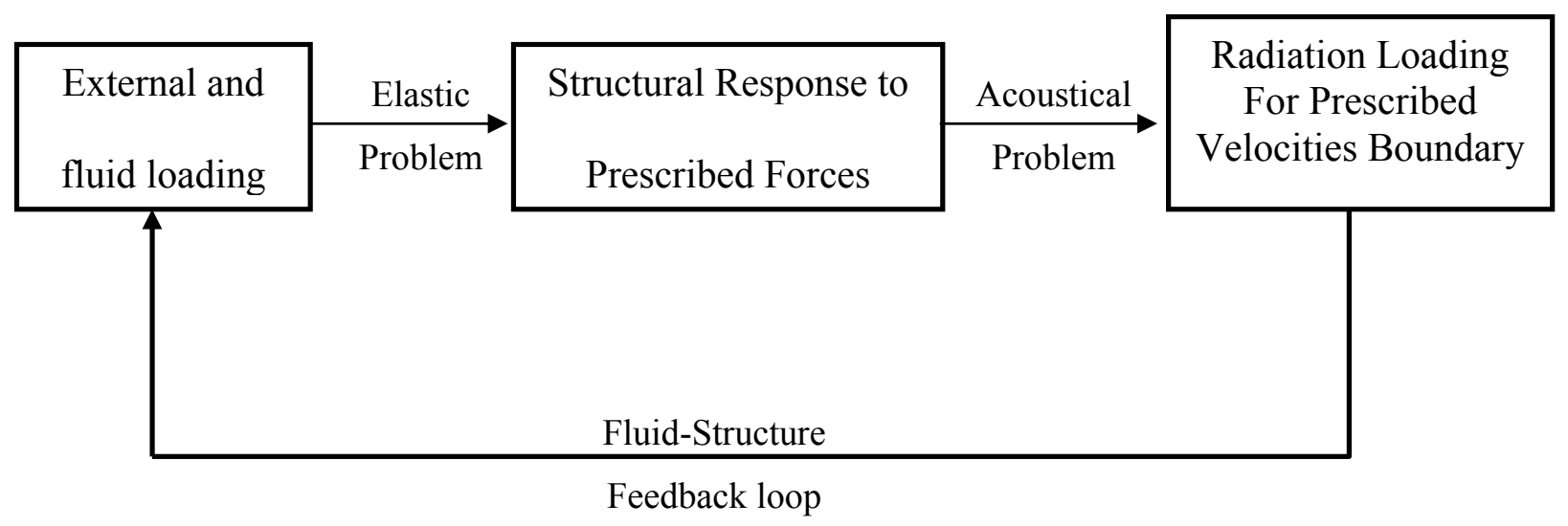

Figure 3.5: Dynamic interaction between the structure and the fluid.

The equation of motion of the system including the fluid loading is written as

$$
\left(\left[L_{c}\right]-\Omega^{2}[I]\right)\left\{\begin{array}{l}
u \\
v \\
w
\end{array}\right\}=\left\{\begin{array}{l}
f_{u} \\
f_{v} \\
f_{w}
\end{array}\right\}-\left\{\begin{array}{c}
0 \\
0 \\
p(x, \theta, a)
\end{array}\right\}
$$

The forcing function $\left\{f_{u}, f_{v}, f_{w}\right\}^{T}$ in equation (3.36) is the external force either for the harmonic point force in the "free tire" model or the contact patch forcing function in the model of the "deformed tire". The last vector in (3.36) represents the acoustic pressure acting on the tire. The acoustic pressure, $p(x, \theta, a)$, on the tire structure is given by equation (3.22) with $G$ defined in (3.35)

$$
p(x, \theta, a)=\varepsilon_{\mathfrak{I}} \int_{0}^{L_{T}} \int_{0}^{2 \pi} i \omega \rho \dot{w}\left(x_{o}, \theta_{o}\right) G\left(x, \theta, a \mid x_{o}, \theta_{o}, a\right) a \mathrm{~d} \theta_{o} d x_{o}
$$


where $\dot{w}\left(x_{o}, \theta_{o}\right)$ is the radial component of the shell velocity response and $G\left(x, \theta, r \mid x_{o}, \theta_{o}, a\right)$ is the Green's function obtained in (3.35).

The solution of the coupled problem is again obtained by expanding the structural response in term of the modes as:

$$
\left\{\begin{array}{l}
u \\
v \\
w
\end{array}\right\}=\sum_{m=1}^{M} \sum_{n=0}^{N}\left[\Phi_{m n}\right] \cdot\left\{\begin{array}{l}
A_{m n}^{(1)} \\
A_{m n}^{(2)} \\
A_{m n}^{(3)}
\end{array}\right\}
$$

where $A_{m n}^{(j)}$ are the structural modal amplitudes including the fluid loading. Then, replacing (3.38) into (3.36), it gives

$$
\left\{\left[L_{c}\right]-\Omega^{2}[I]\right\} \sum_{m=1}^{M} \sum_{n=0}^{N}\left[\Phi_{m n}\right]\left\{\begin{array}{l}
A_{m n}^{(1)} \\
A_{m n}^{(2)} \\
A_{m n}^{(3)}
\end{array}\right\}=\left\{\begin{array}{l}
f_{u} \\
f_{v} \\
f_{w}
\end{array}\right\}-\left\{\begin{array}{c}
0 \\
0 \\
p(x, \theta, a)
\end{array}\right\}
$$

Once again this equation is pre-multiplied by the transpose of the modal matrix and integrated over the surface of the structure as

$$
\int_{0}^{L_{T}} \int_{0}^{2 \pi}\left[\Phi_{r s}\right]^{T}\left\{\left[L_{c}\right]-[\Omega]^{2}[I]\right\} \sum_{m=1}^{M} \sum_{n=0}^{N}\left[\Phi_{m n}\right]\left\{\begin{array}{c}
A_{m n}^{(1)} \\
A_{m n}^{(2)} \\
A_{m n}^{(3)}
\end{array}\right\} a d \theta d x=\int_{0}^{L_{T}} \int_{0}^{2 \pi}\left[\Phi_{r s}\right]^{T}\left\{\left\{\begin{array}{l}
f_{u} \\
f_{v} \\
f_{w}
\end{array}\right\}-\left\{\begin{array}{c}
0 \\
0 \\
p(x, \theta, a)
\end{array}\right\}\right\} a d \theta d x(3 .
$$

The acoustic pressure acting on the surface of the structure $p(x, \theta, a)$ is obtained from (3.37) where the radial velocity component is replaced in terms of the structural modes and the modal amplitudes as follow 


$$
\dot{w}\left(x_{o}, \theta_{o}\right)=i \omega \sum_{m=1}^{M} \sum_{n=0}^{N}\left\{W_{m n}^{(1)}, \quad W_{m n}^{(2)}, \quad W_{m n}^{(3)}\right\}\left\{\begin{array}{c}
A_{m n}^{(1)} \\
A_{m n}^{(2)} \\
A_{m n}^{(3)}
\end{array}\right\} \sin \left(\frac{m \pi}{L_{T}} x_{o}\right) \cos \left(n \theta_{o}\right)
$$

Then,

$$
\begin{gathered}
p(x, \theta, a)=\varepsilon_{\mathfrak{I}} \int_{0}^{L} \int_{0}^{2 \pi} i \omega \rho_{A} i \omega \sum_{m=1}^{M} \sum_{n=0}^{N}\left\{W_{m n}^{(1)}, \quad W_{m n}^{(2)}, \quad W_{m n}^{(3)}\right\}\left\{\begin{array}{c}
A_{m n}^{(1)} \\
A_{m n}^{(2)} \\
A_{m n}^{(3)}
\end{array}\right\} \sin \left(\frac{m \pi}{L_{T}} x_{o}\right) \cos \left(n \theta_{o}\right) . \\
\cdot \sum_{q}^{Q} \sum_{p}^{P} \sum_{l}^{L} \frac{\Psi_{q p l}(x, \theta, a) \Psi_{q p l}\left(x_{o}, \theta_{o}, a\right)}{\Lambda_{q p l}\left(k_{q p l}^{2}-k^{2}\right)} \frac{1}{\varepsilon_{\mathfrak{I}}} a d \theta_{o} d x_{o}
\end{gathered}
$$

Since only the radial velocity component of the structural response couples with the cavity, the cavity pressure will affect in turn mainly the radial structural modes. Replacing (3.42) into (3.40) and solving the integrals explicitly in terms of the unknown modal amplitudes of the radial modes, the following coupled system of equations results

$$
\left[\left[K_{s r}\right]-\Omega^{2}\left[M_{s r}\right]\right] \cdot\left\{A_{s r}\right\}=\left\{f_{s r}\right\}-\Omega^{2} \sum_{q=0}^{Q} \sum_{p=0}^{P} \sum_{l=0}^{L}\left[\alpha_{m n s r q p l}\right] \cdot\left\{A_{s r}\right\}
$$

where $\left[K_{s r}\right]$ and $\left[M_{s r}\right]$ are diagonal matrices and their elements represents the modal stiffness and mass of the radial modes; $\left\{A_{s r}\right\}$ is the vector of modal amplitudes; $\left\{f_{m n}\right\}$ is the vector of modal forces; and $\sum_{q=0}^{Q} \sum_{p=0}^{P} \sum_{l=0}^{L}\left[\alpha_{\text {srmnqpl }}\right]$ is the fluid coupling matrix, which requires the manipulation of six indexes as shown in Appendix E. The second term in the right hand side of equation (3.43) is moved to the other side and the coupled system of equation is solved for the unknown modal amplitudes $\left\{A_{s r}\right\}$. 
The coefficients $\alpha_{\text {srmn }}$ corresponding to the radial component of the matrix $\sum_{q=0}^{Q} \sum_{p=0}^{P} \sum_{l=0}^{L}\left[\alpha_{\text {srmnqpl }}\right]$ are given by the product of the surface integrals as

$$
\alpha_{s r m n}=\frac{\int_{0}^{L_{T}} \int_{0}^{2 \pi} \Phi_{s r}(x, \theta) \Psi_{q p l}(a, x, \theta) a d \theta d x \int_{0}^{L_{T}} \int_{0}^{2 \pi} \Phi_{m n}\left(x_{o}, \theta_{o}\right) \Psi_{q p l}\left(a, x_{o}, \theta_{o}\right) a d \theta_{o} d x_{o}}{\Lambda_{q p l}\left(k_{q p l}^{2}-k^{2}\right)}
$$

The coefficient in (3.44) represents the effect of the $(q, p, l)$ acoustic mode on the $(m, n)$ structural radial mode and reciprocally. One of the advantage of the model developed here is that the interaction of the acoustic and structural modes can be easily determine by inspection of the coefficients in (3.44). For example, if the coefficient $\alpha_{\text {srmn }}$ vanishes implies that the $(q, p, l)$ acoustic does not affect the $(m, n)$ structural mode. It can be observed that the coupling coefficient in (3.44) is the results of two independent integrations, i.e. in the axial and azimuth directions. Thus, inspection of these integrations can be performed independently. Tables 3.1 and 3.2 shows the results of the coupling analysis for the $(0,1,0)$ acoustic mode with several structural modes in the radial and azimuth directions, respectively. In Table 3.1, the axial pressure variation of the $(0,1,0)$ mode on the shell $(r=a)$ and the axial variation of the structural modes are illustrated. From inspection, it is trivial to find that the inner product of these axial variations show that the $(0,1,0)$ acoustic mode will couple with only the $(1,1)$ and $(1,3)$ structural modes. Table 3.2 shows the coupling results on the azimuth direction for the $(0,1,0)$ acoustic mode and the $(1,1),(1,2)$, and $(1,3)$ structural modes. Simple inspection of this table shows that the $(0,1,0)$ mode couples with only the $(1,1)$ structural mode in the azimuth direction. It is easy to find that acoustic and structural modes that have the same azimuth variation will be coupled. Thus, the acoustic mode $(0,1,0)$ can couple with the $(1,1),(3,1)$, $(5,1)$ and so forth. 
Table 3.1: Description of the effect of axial variation on the coupling between structural and acoustic modes of same azimuth variation.

\begin{tabular}{|c|c|c|c|}
\hline & \multicolumn{3}{|c|}{ Structural $(\mathrm{m}(\mathrm{x}), \mathrm{n}(\theta))$} \\
\hline $\begin{array}{c}\text { Acoustic } \\
(\mathrm{q}(\mathrm{x}), \mathrm{p}(\theta), 1(\mathrm{r}))\end{array}$ & AIIIIIIIIn & 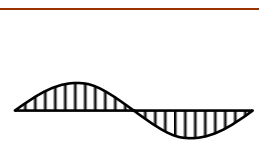 & 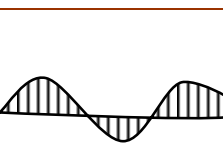 \\
\hline 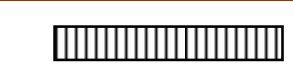 & $(1,1)$ & $(2,1)$ & $(3,1)$ \\
\hline$(0,1,0)$ & $\begin{array}{l}\text { Coupling } \\
\alpha_{1111010} \neq 0\end{array}$ & $\begin{array}{c}\text { No coupling } \\
\alpha_{1212010}=0\end{array}$ & $\begin{array}{l}\text { Coupling } \\
\alpha_{1313010} \neq 0\end{array}$ \\
\hline
\end{tabular}

Table 3.2: Description of the effect of in the circumferential direction on the coupling between structural and acoustic modes.

\begin{tabular}{|c|c|c|c|}
\hline & \multicolumn{3}{|c|}{ Structural $(\mathrm{m}(\mathrm{x}), \mathrm{n}(\theta))$} \\
\hline $\begin{array}{c}\text { Acoustic } \\
(\mathrm{q}(\mathrm{x}), \mathrm{p}(\theta), 1(\mathrm{r}))\end{array}$ & $(1,1)$ & $(1,2)$ & $(1,3)$ \\
\hline & $\begin{array}{c}\text { Coupling } \\
\alpha_{1111010} \neq 0\end{array}$ & $\begin{array}{c}\text { No Coupling } \\
\alpha_{1212010}=0\end{array}$ & $\begin{array}{c}\text { No Coupling } \\
\alpha_{1313010}=0\end{array}$ \\
\hline$(0,1,0)$ &
\end{tabular}

The modal amplitudes for the radial modes are obtained from equation (3.43), and the structural response is then obtained from (3.38) and then the acoustic response is computed using (3.37).

Equation (3.43) represents the following coupled matrix equation 
$\left[\begin{array}{cccc}{\left[H_{10}\right]} & {[0]} & \cdots & {[0]} \\ {[0]} & {\left[H_{11}\right]} & \cdots & {[0]} \\ \vdots & \vdots & \ddots & \vdots \\ {[0]} & {[0]} & \cdots & {\left[H_{1 N}\right.}\end{array}\right.$

$$
\begin{array}{cccc}
{\left[H_{20}\right]} & {[0]} & \cdots & {[0]} \\
{[0]} & {\left[H_{21}\right]} & \cdots & {[0]} \\
\vdots & \vdots & \ddots & \vdots \\
{[0]} & {[0]} & \cdots & {\left[H_{2 N}\right]}
\end{array}
$$
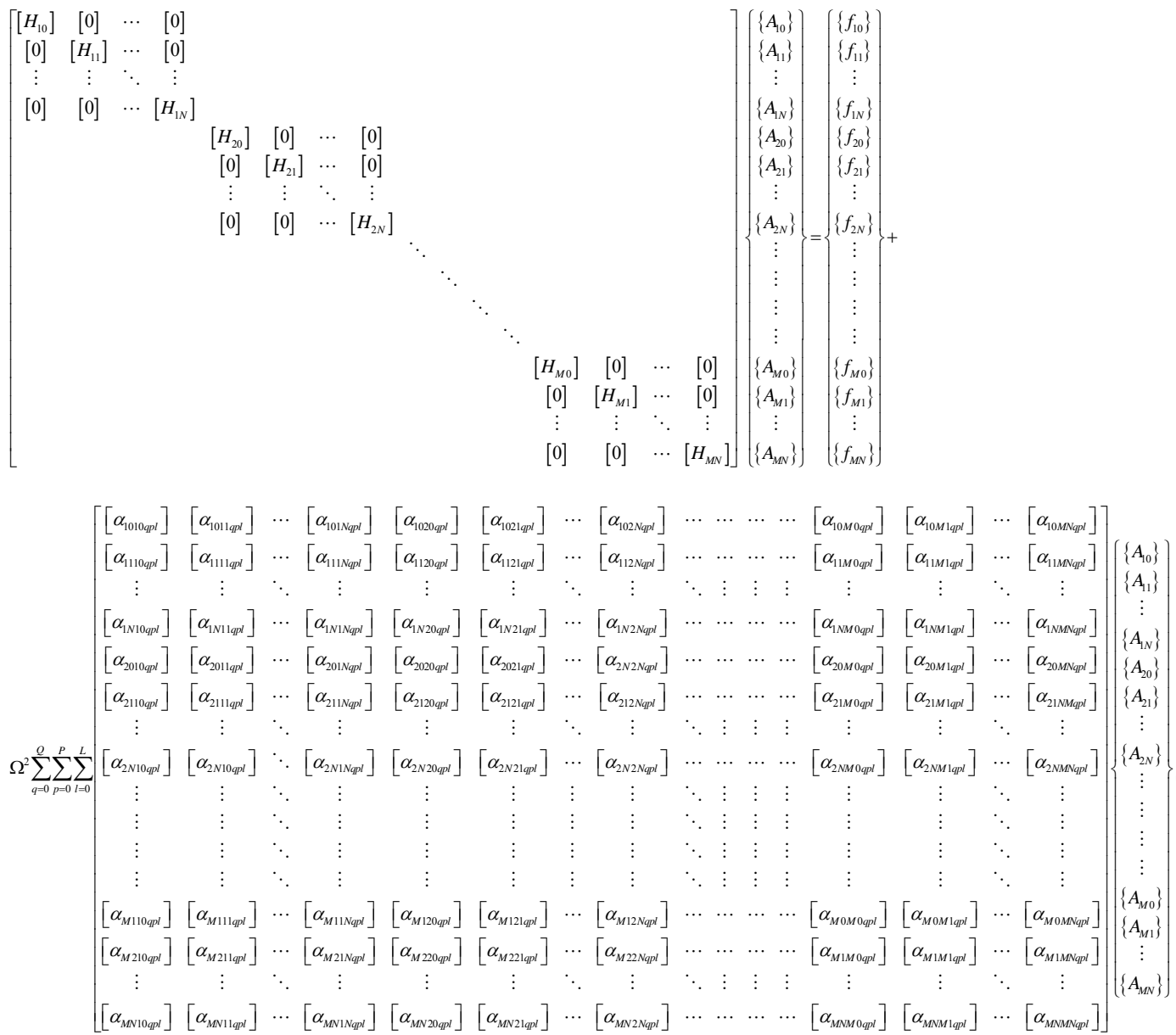

where in the last equation

$$
\left[H_{s r}\right]=\left[\left[K_{s r}\right]-\Omega^{2}\left[M_{s r}\right]\right]
$$

\subsection{FREE TIRE NUMERICAL SIMULATIONS}

In this section, a numerical simulation for the coupled tire model will be presented. The analytical tire model will also be validated with experimental results. The material properties of the tire are again given by Table 2.1 in Chapter 2 and the tire size is 195/65 R15. 
Table 3.3 shows the acoustic cavity resonance frequencies for a range between 0 to 1000 Hz. The acoustic natural frequency for the mode $\Psi_{010}$ is highlighted in the table because is the only acoustic mode in the frequency range of interest that couples with the structure dynamics. From Table 2.2, this acoustic mode will couple with only the $(1,1)$ radial mode.

Table 3.3: Tire acoustic cavity natural frequencies for tire 195/65 R15 between [0-1000]

$\mathrm{Hz}$

\begin{tabular}{|c|c|c|c|}
\hline $\mathbf{q}$ & $\mathbf{p}$ & $\mathbf{l}$ & frequency $[\mathrm{Hz}]$ \\
\hline 0 & 0 & 0 & 0.0 \\
\hline 0 & 1 & 0 & 232.6 \\
\hline 0 & 2 & 0 & 465.1 \\
\hline 0 & 3 & 0 & 697.6 \\
\hline 1 & 0 & 0 & 879.5 \\
\hline 1 & 1 & 0 & 909.7 \\
\hline 0 & 4 & 0 & 930.2 \\
\hline 1 & 2 & 0 & 994.9 \\
\hline
\end{tabular}

The in vacuo and coupled responses were computed and compared over the frequency range of $0-400 \mathrm{~Hz}$. The analysis included 25 structural modes and 5 acoustic modes. Figure 3.6 and 3.7 show the modal amplitudes for the $\Phi_{11}^{(1)}$ and $\Phi_{31}^{(1)}$ radial structural modes with and without acoustic coupling, respectively. The tire radial mode $\Phi_{11}^{(1)}$, and the acoustic mode $\Psi_{010}$ are coupled as shown in these figures. It is clear that the effect of the tire acoustic cavity mode in the structural response is significant even though the acoustic resonance is not very close to the structural resonance, i.e. not tuned. Since the acoustic system is modeled without acoustic damping, the "slight" damping in the acoustic resonance is coming from the tire structure due to the coupling process. It can be observed that this small amount of damping do not help enough to control the adverse effect of the acoustic cavity in the modal amplitude response, i.e., Figure 3.6 and 3.7. 


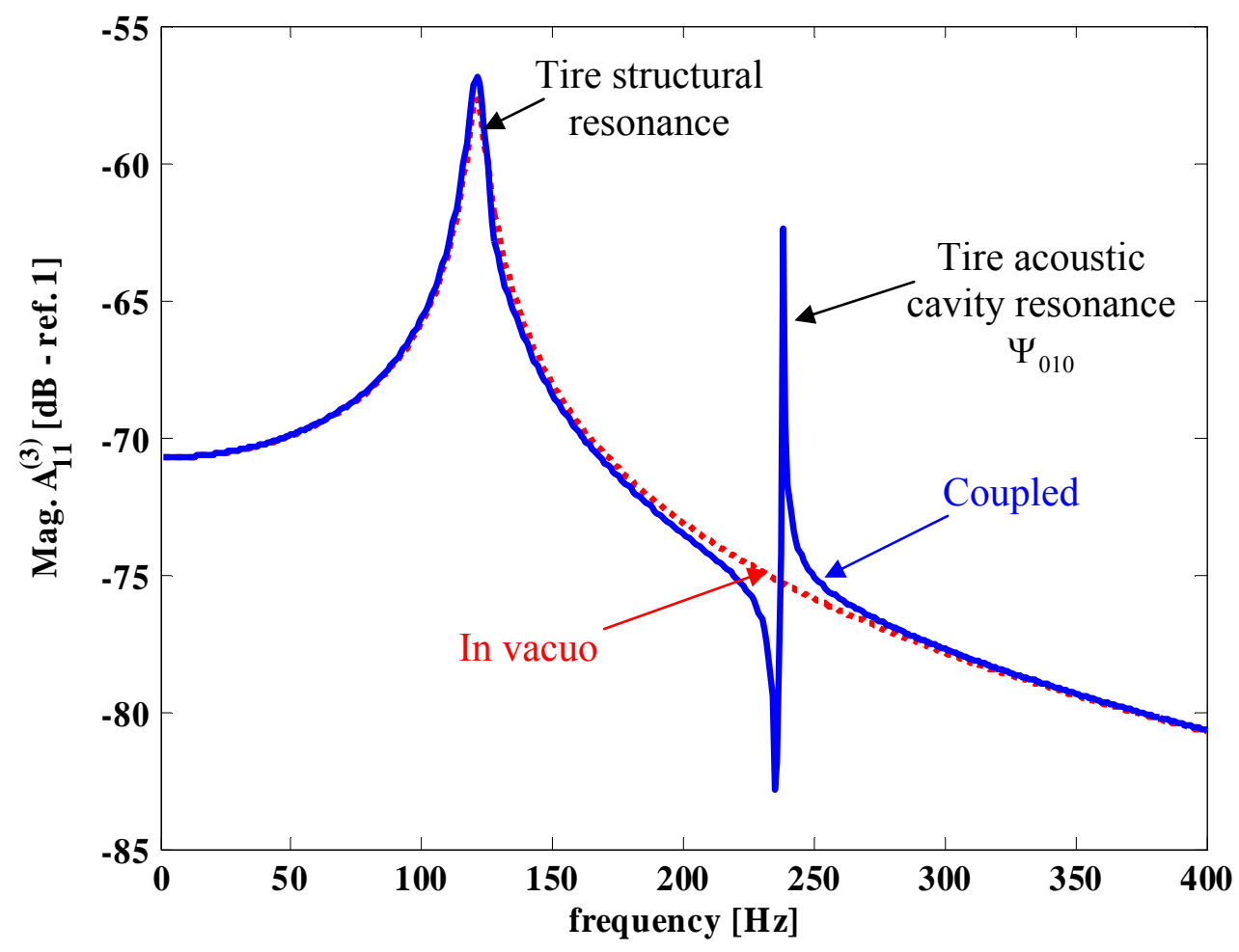

Figure 3.6: Radial Modal Amplitude for mode $\Phi_{11}^{(1)}$.

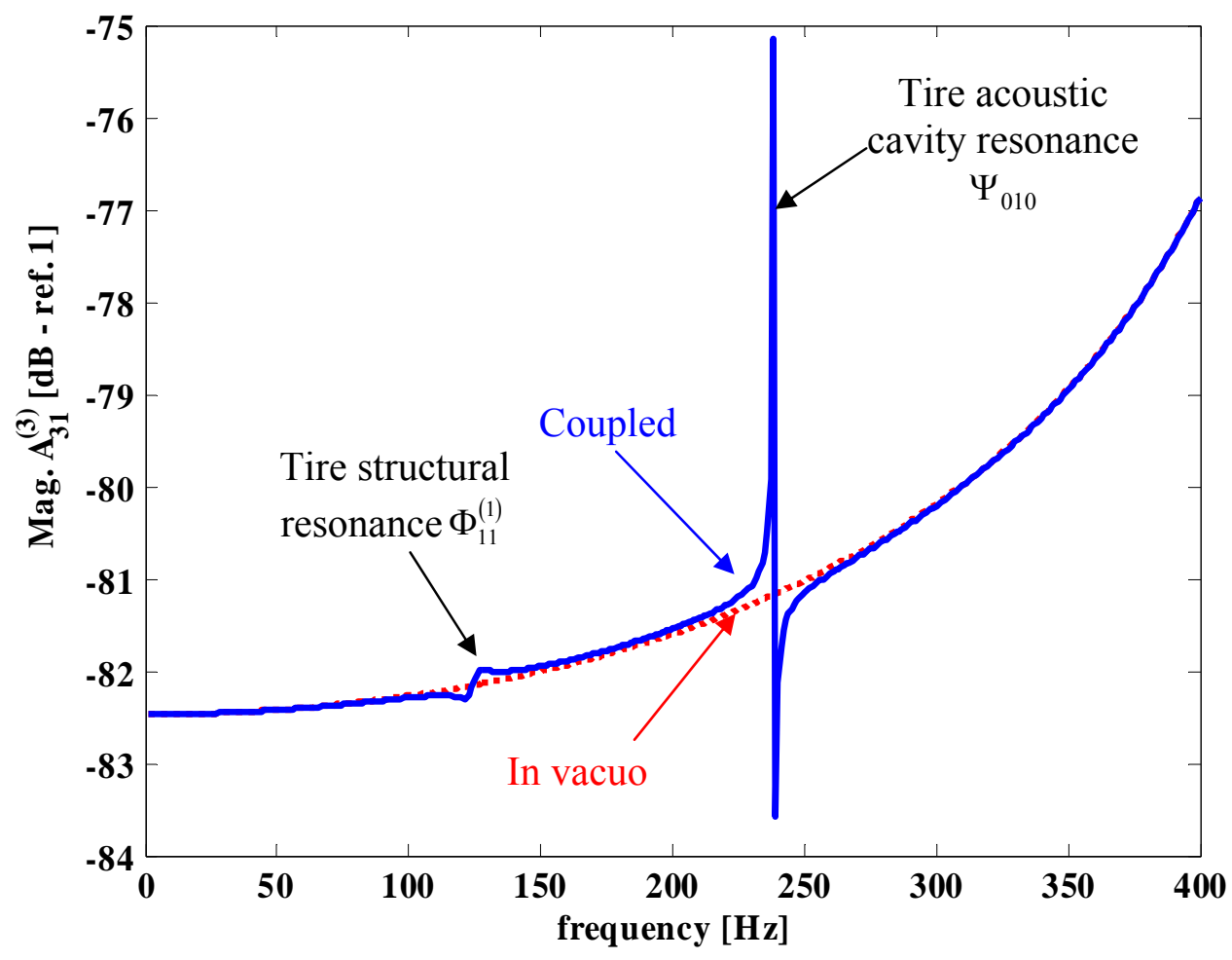

Figure 3.7: Radial Modal Amplitude for mode $\Phi_{31}^{(1)}$. 
It is interesting to note that the relatively high damping of the tire structure does not damp out the energy in the acoustic resonance. Figure 3.7 also reveals the coupling of the structural modes $\Phi_{11}^{(1)}$ and $\Phi_{31}^{(1)}$ by the acoustic mode.

The resultant force and moment at the spindle were also computed. Figure 3.8 and Figure 3.9 show the spindle force and moment with and without the acoustic cavity effect, respectively. Again they show that the internal acoustic cavity resonance effects are important and thus they should be accounted for in the prediction of vehicle interior noise.

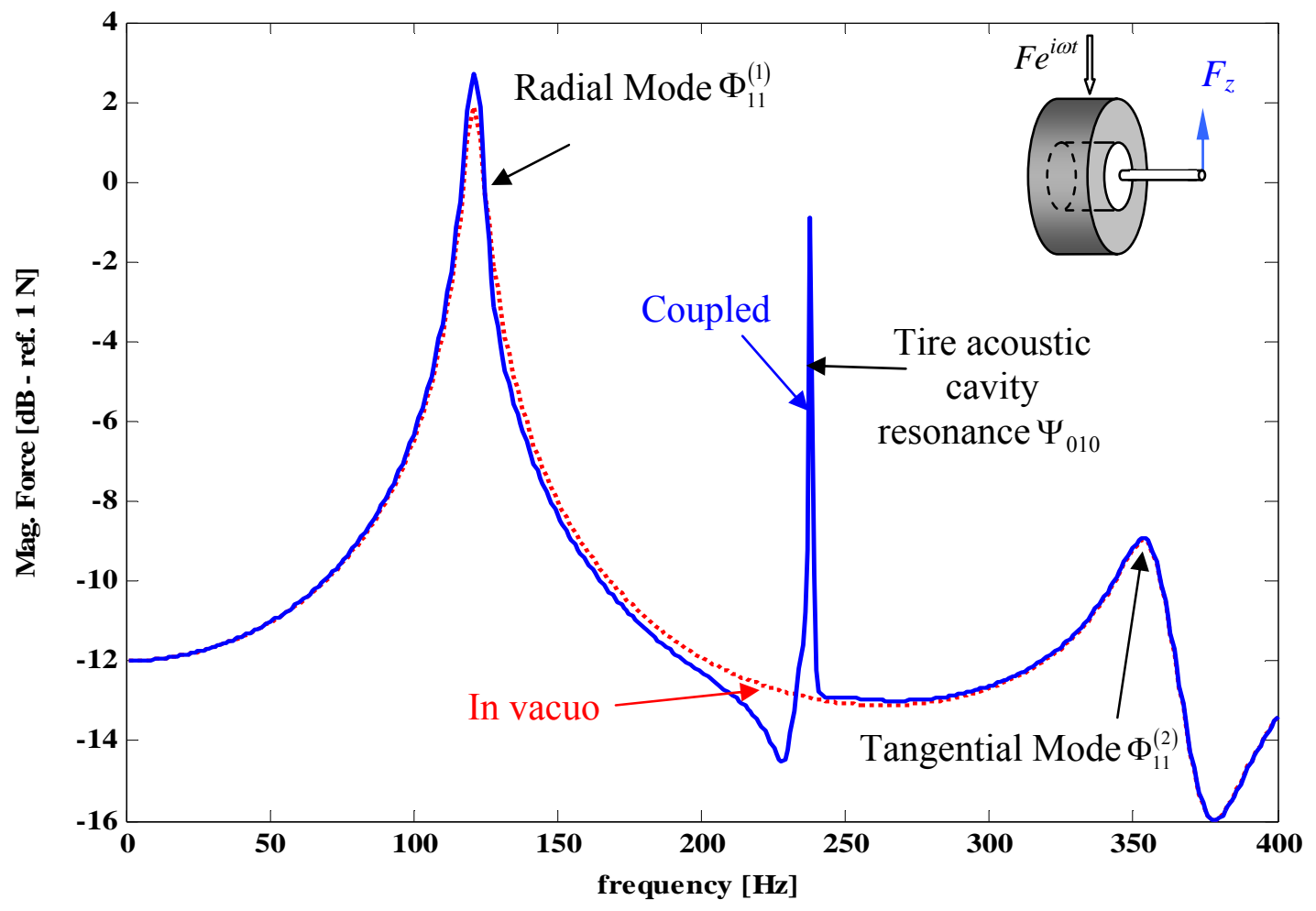

Figure 3.8: Vertical force $\mathrm{F}_{\mathrm{z}}$ at the spindle. 


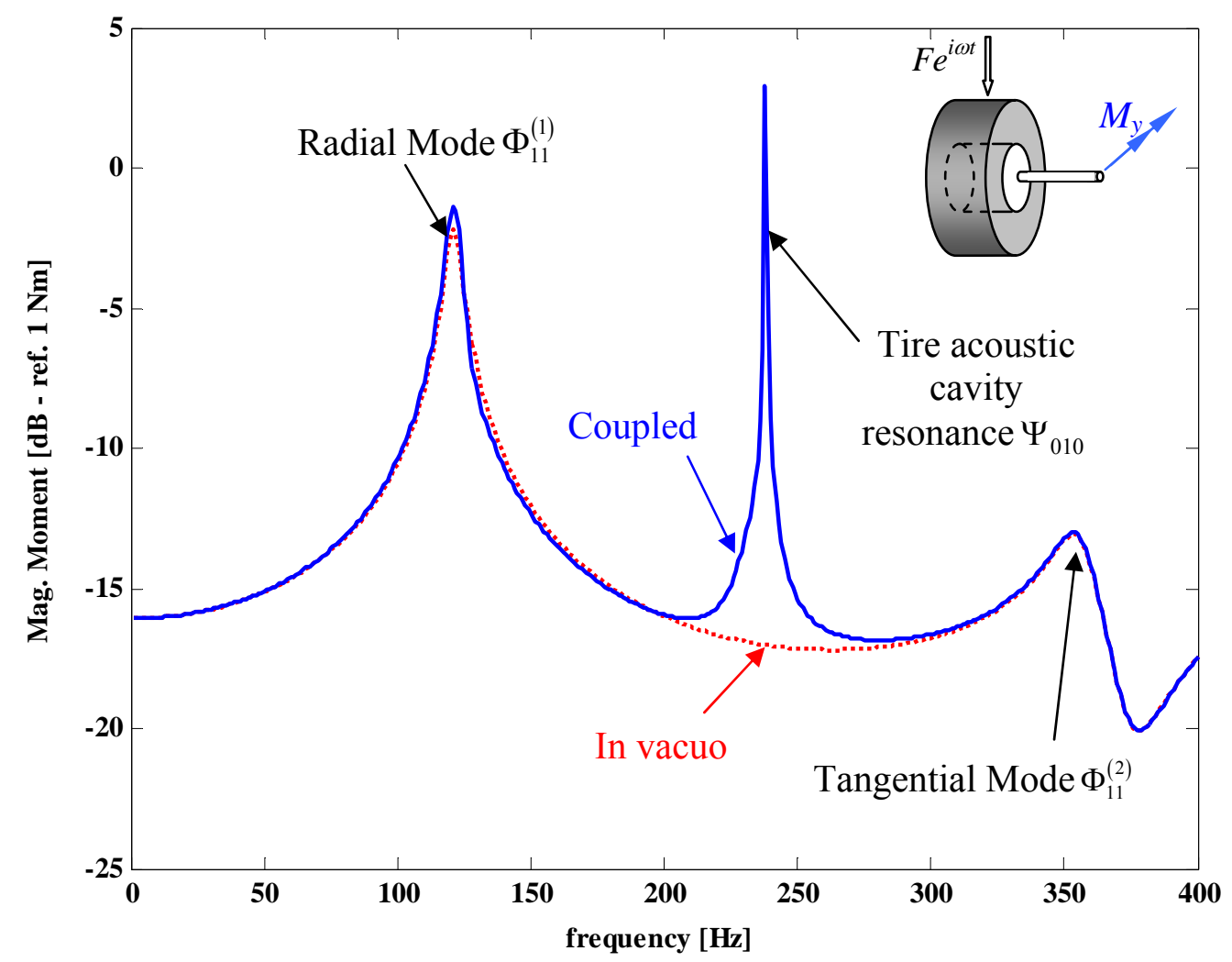

Figure 3.9: Horizontal moment $\mathrm{M}_{\mathrm{y}}$ at the spindle.

Figure 3.10 shows the radial displacement at the external force location with and without acoustic coupling. The cavity resonance effect is present for the coupled case at $232 \mathrm{~Hz}$ due to the influence of the acoustic mode $\Psi_{010}$.

Figure 3.11 includes three curves corresponding to the in vacuo model in red dotted line, the coupled model in blue solid line, and results from a test measurement performed by Yamauchi and Akiyoshi (2002) in a green dash-dotted line. The gain factor to match the acoustic cavity resonances is 0.1775 . The results from this simple analytical model show qualitatively similar trends as the experimental observation, i.e. blue line for coupled and green line for experimental results. The green line corresponding to the experimental data shows also the presence of the rigid body motion, which is not accounted for the model. The dynamic of the tire structure is clearly represented and the effect of the tire acoustic cavity is very well predicted. 
These results provide a validation of the close model approach undertaken here as a valid tool to capture the behavior of the tire dynamics including the acoustic cavity.

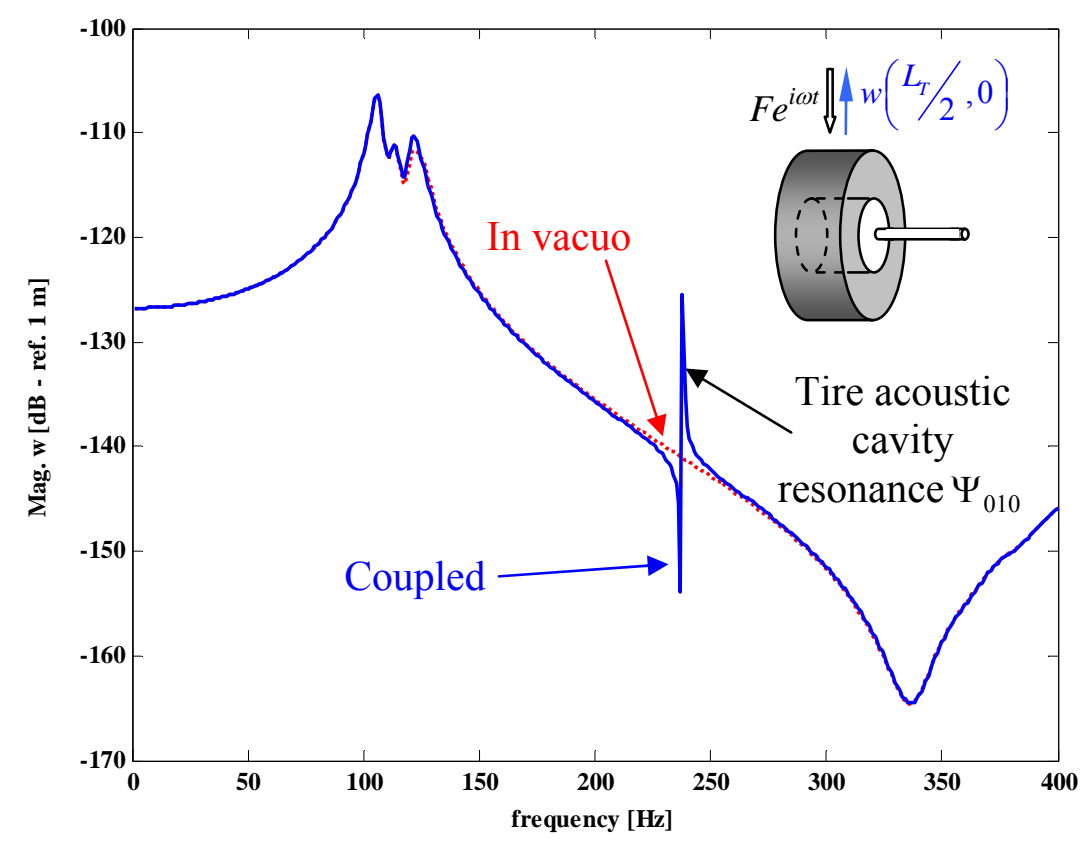

Figure 3.10: Radial displacement for the free tire model at the external force location.

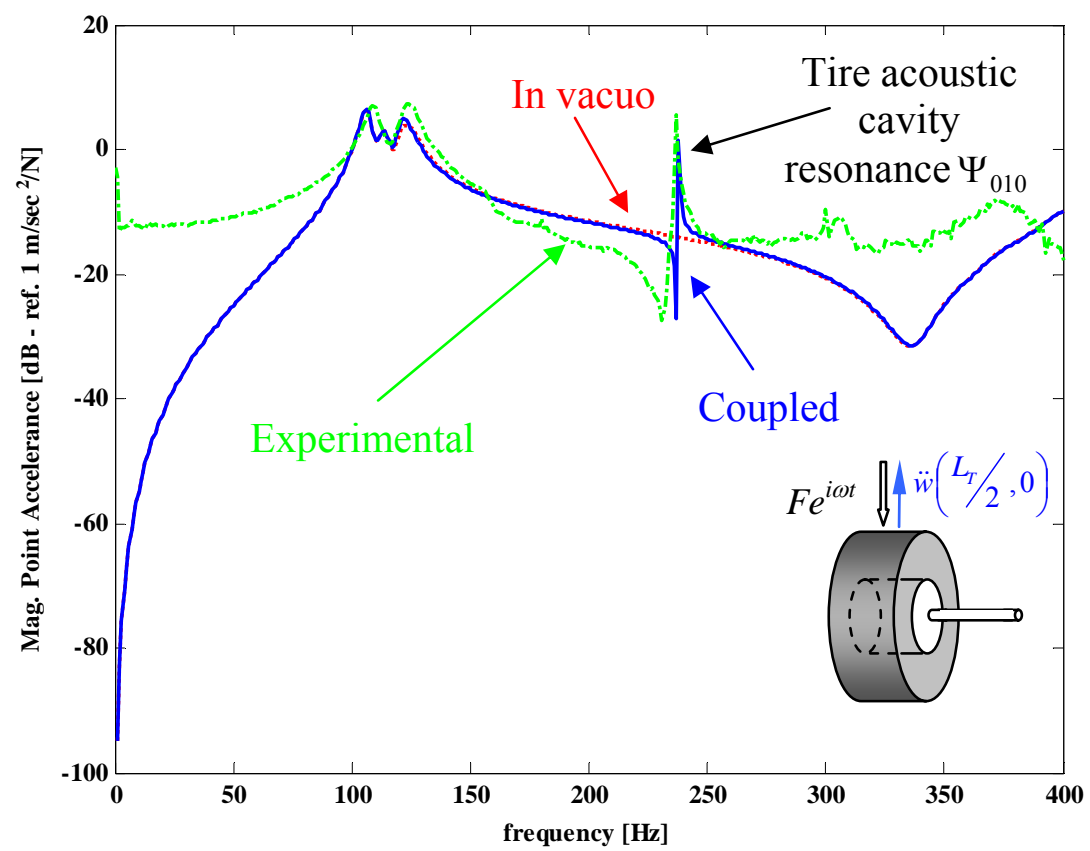

Figure 3.11: Point accelerance for the free tire model at the external force location. 


\subsection{DEFORMED TIRE NUMERICAL SIMULATIONS}

In this section the results for the case of the deformed tire are presented. The contact patch depth was assumed to be $\bar{h}=0.01 \mathrm{~m}$ and the harmonic oscillating amplitude is $\varepsilon=1 \times 10^{-5} \mathrm{~m}$. The tire material properties are given in the Table 2.1 and the tire size is 195/65 R15. The model is also validated with experimental results provided by Yamauchi and Akiyoshi (2002).

Figure 3.12 and 3.13 illustrate the resulting radial modal amplitudes for the modes $\Phi_{11}^{(1)}$ and $\Phi_{31}^{(1)}$ respectively. The acoustic cavity resonance is present in the coupled tire response. In the "deformed tire" model, the resonance of the acoustic cavity is larger than the structural resonance, i.e. Figure 3.12 and 3.13. Note that in the case of the "free tire", the structural resonance is larger than the acoustic resonance, i.e. Figure 3.6 and 3.7.

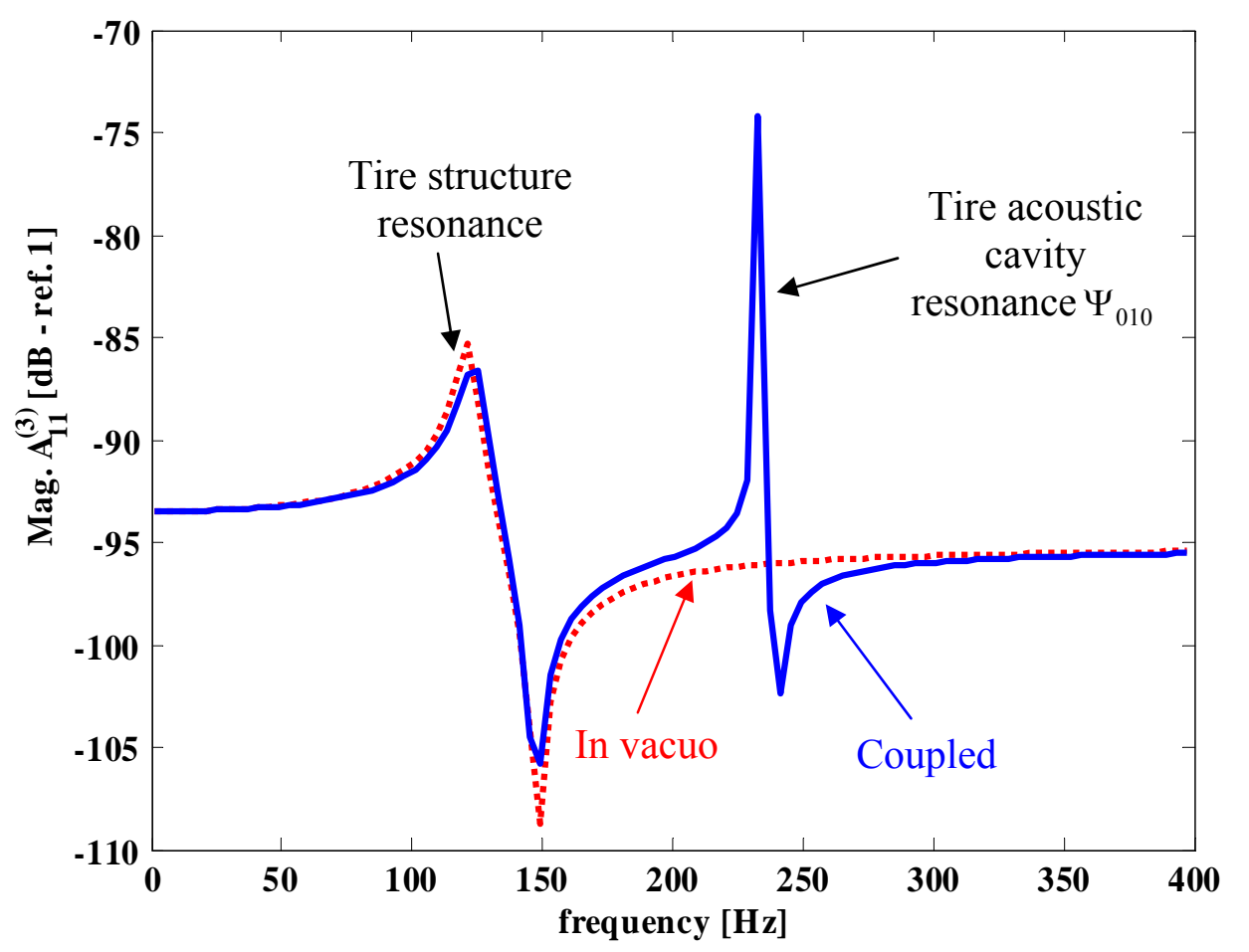

Figure 3.12: Radial Modal Amplitude for mode $\Phi_{11}^{(1)}$. 


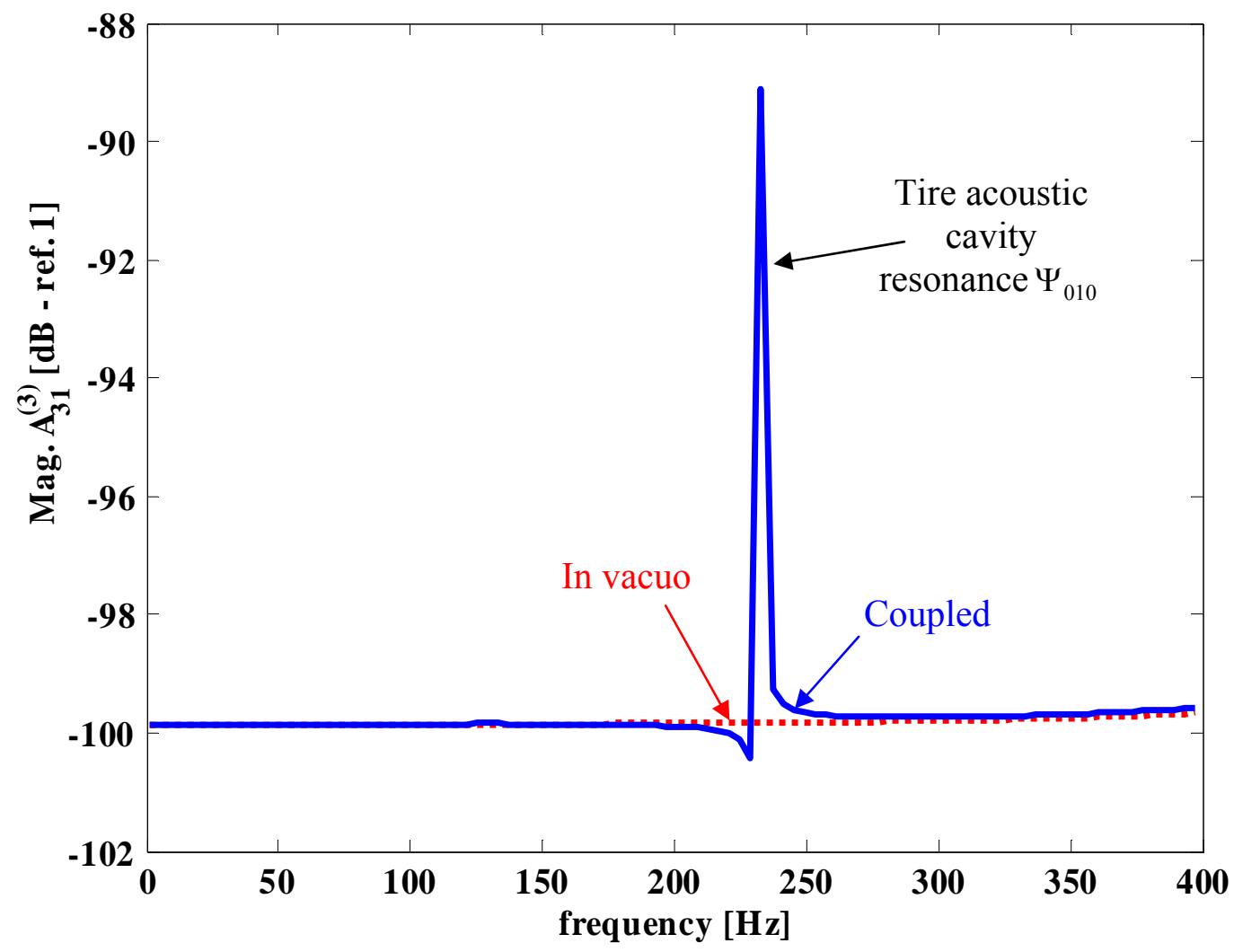

Figure 3.13: Radial Modal Amplitude for mode $\Phi_{13}^{(1)}$.

The force and moment at the spindle for the deformed model are shown in Figure 3.14 and 3.15, respectively. The cavity resonance effect relative to the structural resonance is much significant in the "deformed tire" forces than in the "free tire", i.e. from comparison of Figure 3.8 and 3.9 to Figure 3.14 and 3.15, respectively. 


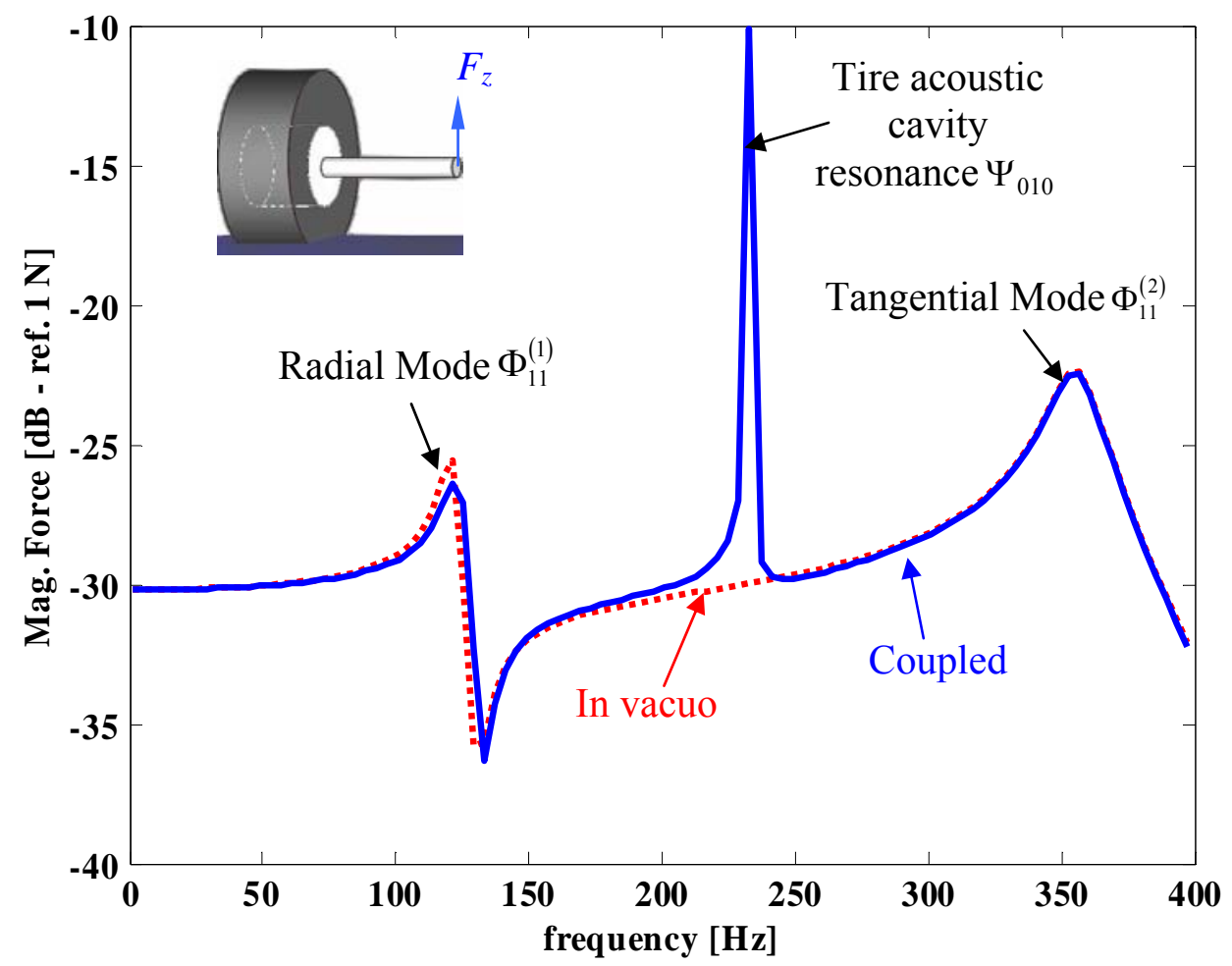

Figure 3.14: Vertical force $\mathrm{F}_{\mathrm{z}}$ at the spindle.

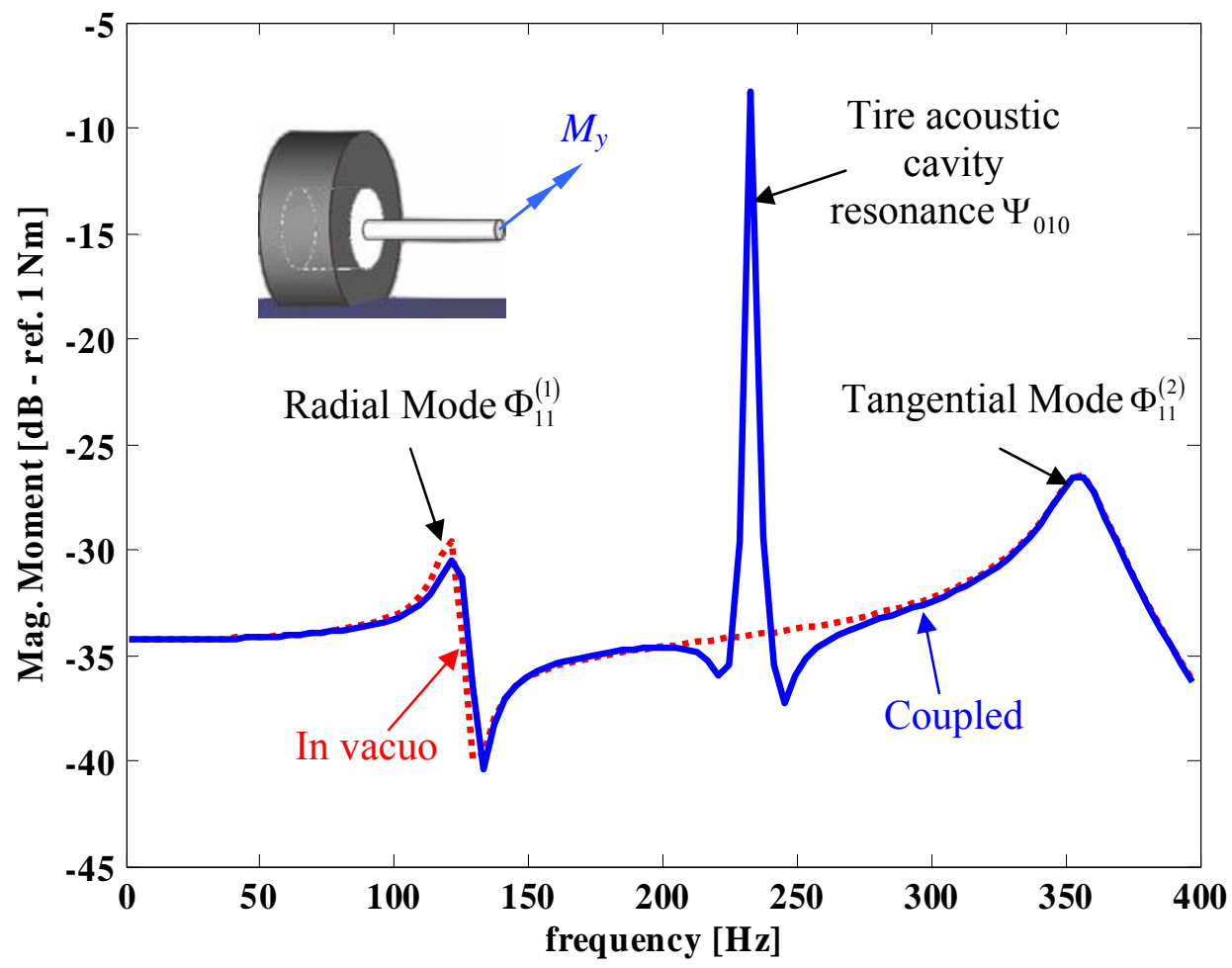

Figure 3.15: Horizontal moment $\mathrm{M}_{\mathrm{y}}$ at the spindle. 
The radial displacement at $\left(x=\frac{L_{T}}{2}, \theta=0\right)$ can be seen in Figure 3.16. From comparison of Figure 3.16 to Figure 3.10, it can be observed that the acoustic resonance in the "deformed tire" has a much larger effect on the tire response in contrast to the "free tire".

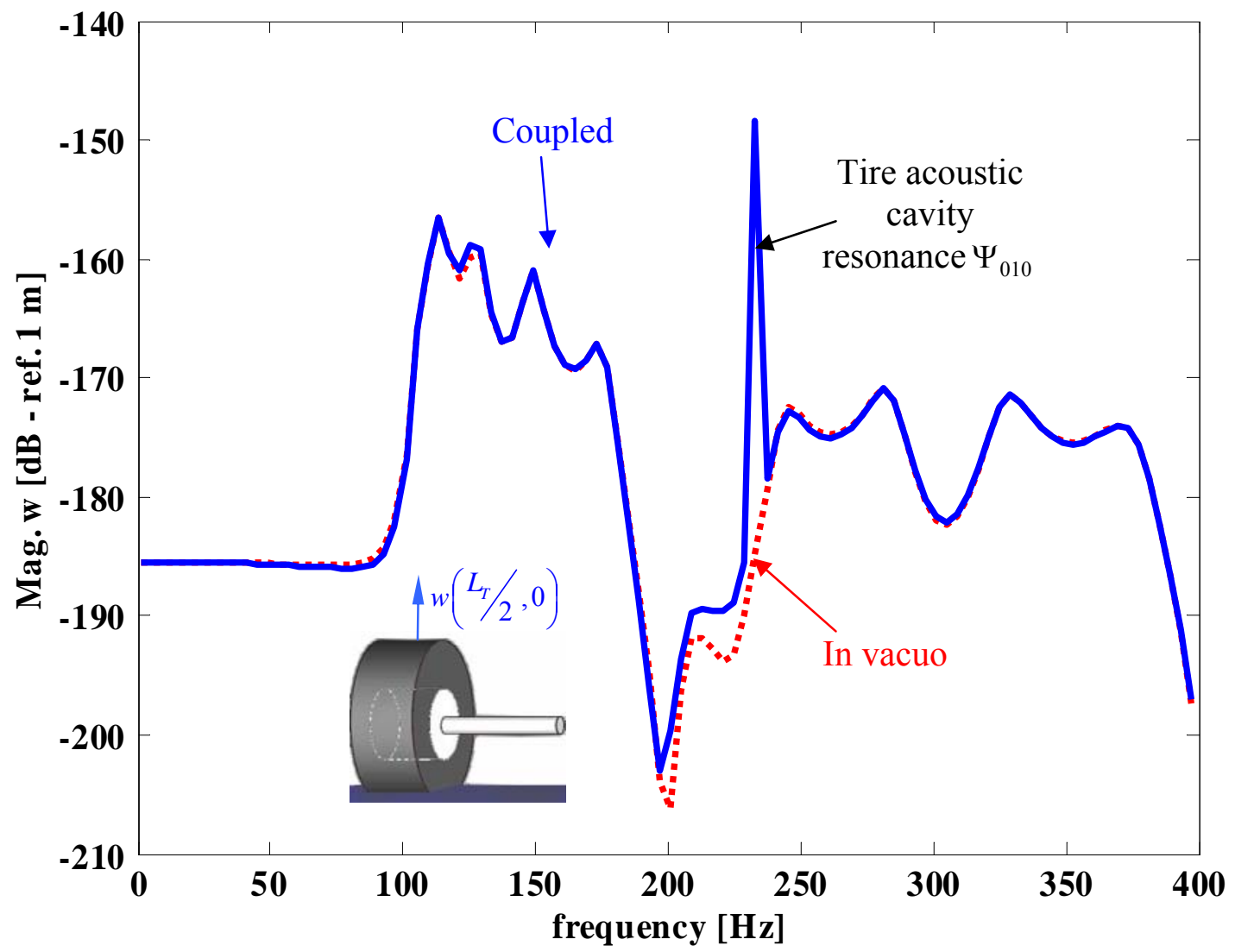

Figure 3.16: Radial displacement for the deformed tire model at location $\left(x=\frac{L_{T}}{2}, \theta=0\right)$.

Figure 3.17 shows the point accelerance given for the "deformed tire" model. The gain factor used in this case is 0.0041 in order to match the acoustic cavity resonances. In this figure the results for the in vacuo and coupled cases are shown. For the coupled case, the experimental result obtained by Yamauchi and Akiyoshi (2002) is also included. It can again be observed that the coupled "deformed tire" model predicts the effect of the tire 
acoustic cavity very well and thus validate the proposed model.

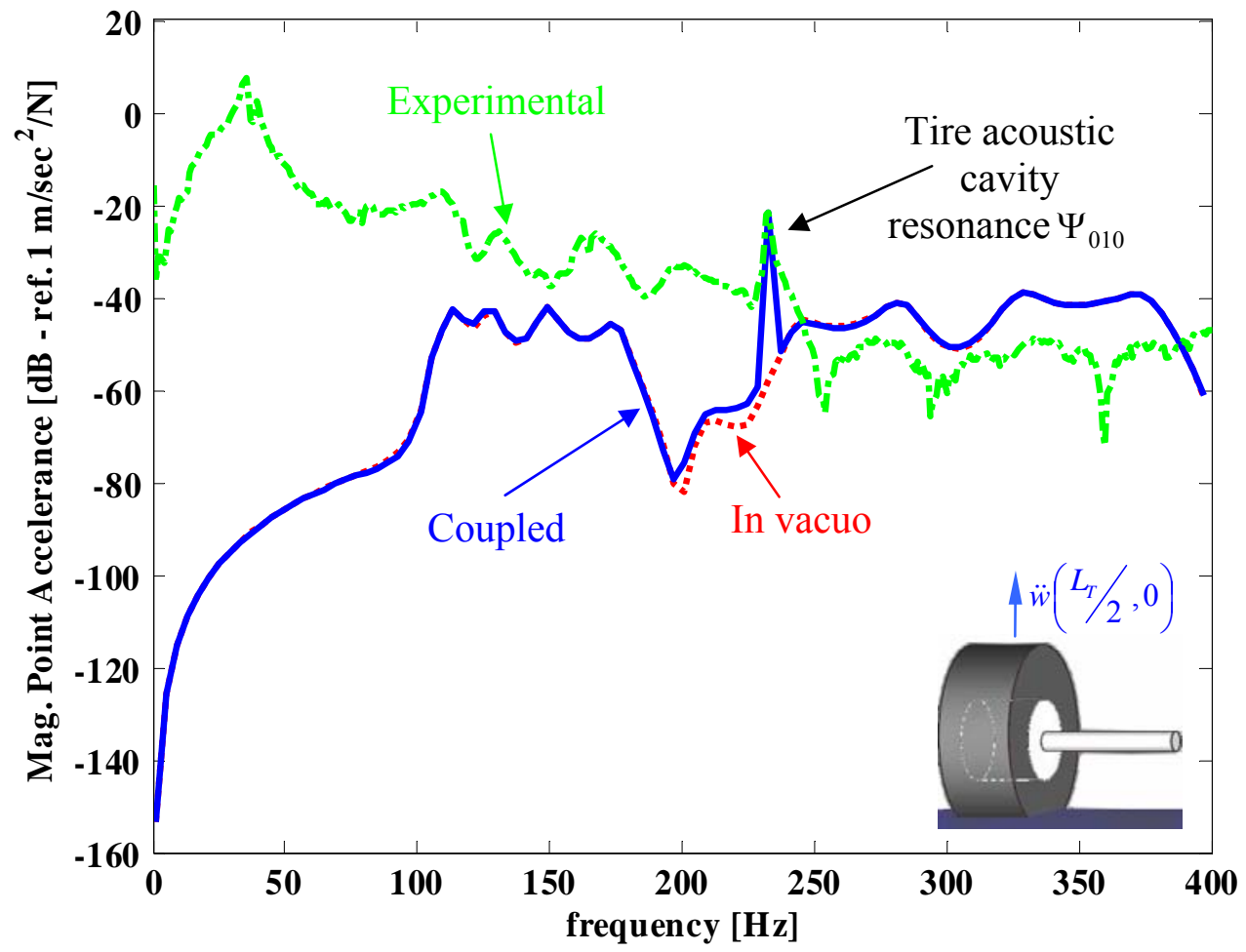

Figure 3.17: Point accelerance for the deformed tire model at location $\left(x=\frac{L_{T}}{2}, \theta=0\right)$.

As a concluding remark for this chapter, the results presented by the models allow to clearly identifying the coupling mechanisms between the structural and acoustic modes. In addition, the results show that the acoustic cavity resonances have a significant effect on the response of the system, i.e. the cavity resonances results in important forces and moments at the spindle, which are the source of noise in the vehicle interior. 


\section{CHAPTER 4: CONTROL OF THE TIRE ACOUSTIC CAVITY RESONANCE}

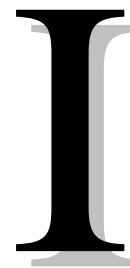

$\mathrm{n}$ the previous chapter, the influence of the acoustic cavity resonance on the spindles forces was shown to be very important. The acoustic cavity dynamics results in a sharp increase of the spindle forces around the tire cavity resonance. These forces are transmitted to the rest of the car structure and contribute adversely to the vehicle interior noise. Therefore, the tire cavity resonance effect must be reduced in order to control the tire contribution to the structural born noise.

In this chapter, the analysis and modeling of two feasible approaches to control the tire acoustic cavity resonances are proposed and investigated. The approaches are analytically modeled and included into both the "free" and "deformed" tire formulations.

The first approach is the incorporation of secondary acoustic cavities to detune and damp out the main tire cavity resonance. The second approach is the addition of damping directly into the tire cavity. The damping is achieved by placing viscoelastic screens into the tire acoustic cavity. The use of viscoelastic screens is a novel technique to control the acoustic resonance.

The control concepts are explained in more detail in the next sections. The modeling of the secondary cavities technique is developed in section 4.2. The numerical simulations related to this technique are shown in section 4.3. Then, in section 4.4 the viscoelastic screen approach is presented. 


\subsection{SECONDARY CAVITIES CONTROL APPROACH}

The secondary cavity control approach is similar to the concept of Dynamic Vibration Absorber (DVA) approach used in structures. In the DVA, a secondary dynamic (mass, spring, damper) system is used to reduce the vibration level of a primary system (Rao, 1990 and Meirovitch, 2001). The DVA concept schematic is shown in Figure 4.1. The vibration level reduction is achieved by detuning, i.e. shifting of the resonance, and adding damping to the primary system. Figure 4.1 shows that the main resonance of the primary system "splits” in two when the secondary system is added and tuned to that resonance frequency. The frequency range in Figure 4.1 is normalized with respect to the resonance frequency of the primary system. The resonance shift is governed by the mass of the secondary system, i.a. DVA mass. The amplitude of the resonances of the new coupled system is controlled by the amount of damping incorporated in the absorber.

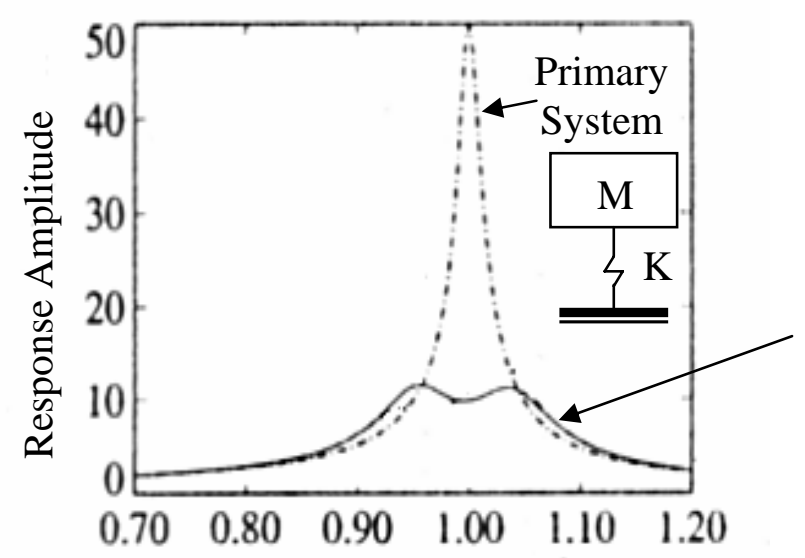

Acoustic

DVA

Normalized Frequency

Figure 4.1: Dynamic Vibration Absorber.

The DVA concept is applied to detune the tire acoustic cavity (primary system) by using secondary cavities as illustrated in Figure 4.2. The DVA concept to the cavity acoustic problem is ideal because there is a need to control a single cavity mode, i.e. the $(0,1,0)$ 
acoustic mode. In practice, the secondary cavities can be added to the wheel of the tire as shown in Figure 4.2a, i.e. attached and conformal to the wheel. The best location for the cavity is at the anti-node of the acoustic mode shape. Since this is not feasible due to the rolling of the tire, more than a single cavity is needed. Therefore, the effect of the rotation of the tire is accounted by using two secondary cavities placed $90^{\circ}$ apart from each other in the azimuth direction. This amount of angular separation is selected because the acoustic mode shape that need to be controlled has an azimuth variation of $\cos (\theta)$, i.e. $p=1$. Thus, as the tire rotates there is at least one cavity that is effective in controlling the cavity resonance, i.e. as one of the secondary cavity is located at a node the other is at an antinode and able to detune the main tire cavity resonance. The tire main and secondary cavities are interconnected by openings which are referred here as the “coupling interfaces". To introduce damping in the system, sound absorptive screens such as wiremesh, perforate plates, and so forth can be implemented at these interfaces. In addition to the damping, the absorptive screens will also introduce a masslike impedance, i.e. mass. The resistance of the screens will introduce damping of the acoustic resonances and consequently reduce their magnitude.

The formulation of the tire cavity coupled to secondary cavities is now presented. The modeling of the tire main cavity coupled to the secondary cavities can be accomplished using two approaches. The first option is to find the new eigenproperties for the coupled tire-secondary cavity as single system assuming rigid boundary conditions. The new eigenproperties can then be used to construct the Green's function which in turn can be directly used in the same formulation presented in chapter 3. The difficulty with this approach is that the modeling of the screen at the coupling interfaces is not straightforward. Modeling of the complex impedance at the coupling interfaces would lead to complex eigenproperties. 


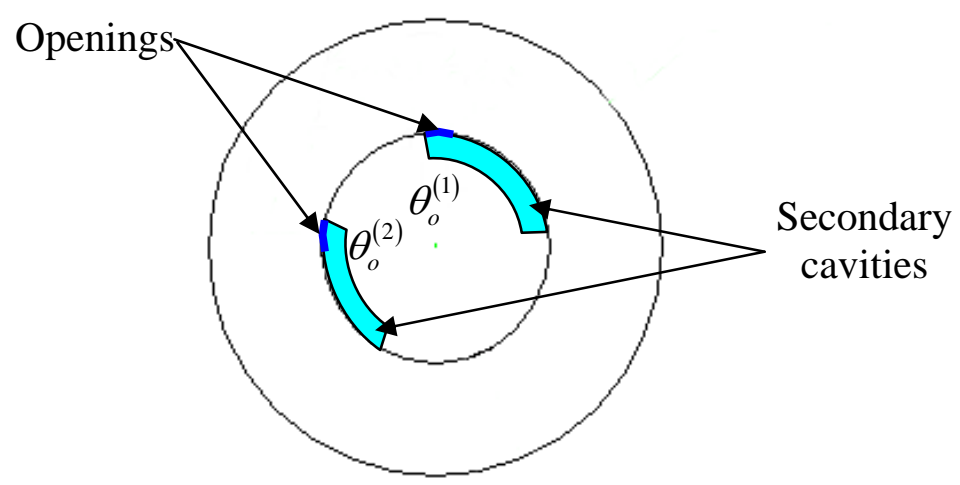

(a)

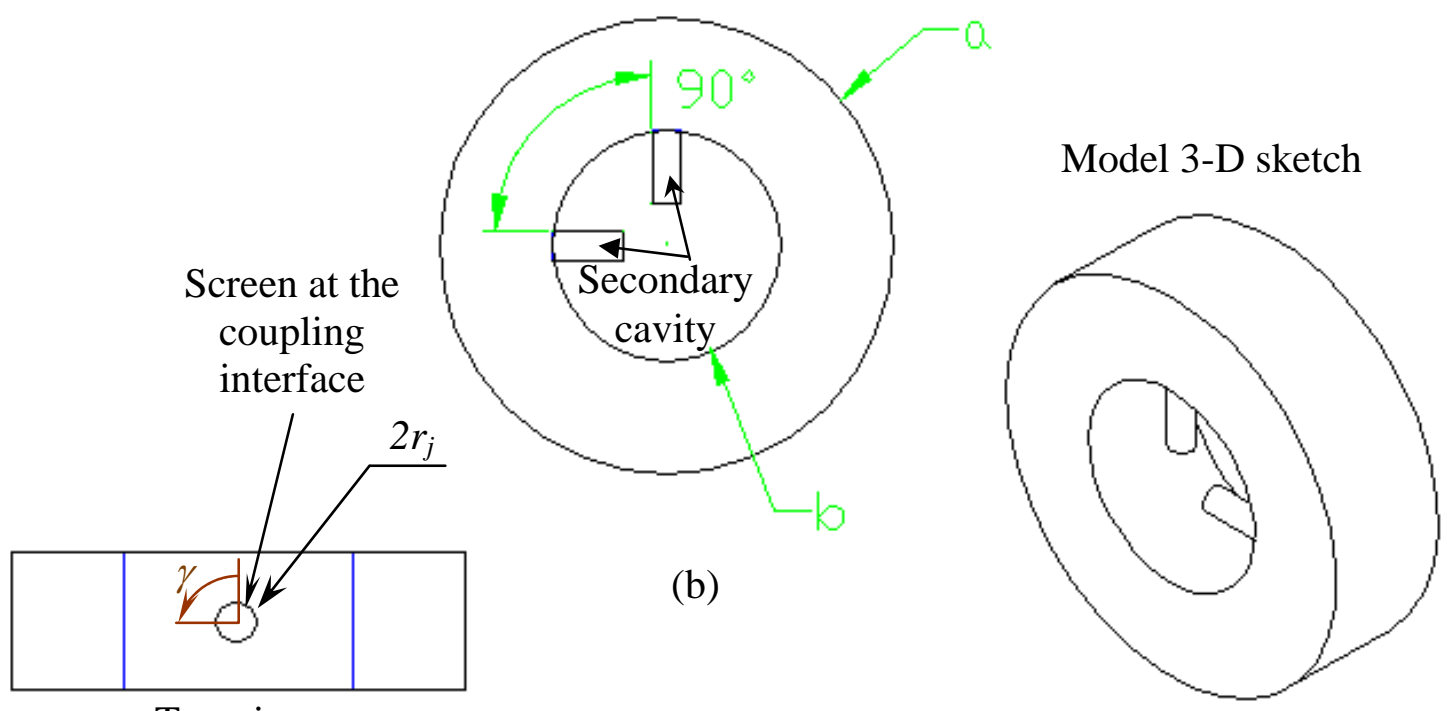

Top view

Figure 4.2: Tire and secondary cavities acoustic modeling.

The second method is illustrated in Figure 4.3 and involves first separating the secondary cavities from the tire cavity. Then, the effect of the secondary cavities on the tire cavity is considered or modeled as an additional moving boundary in the formulation, i.e. in addition to the elastic shell motion. This approach has two main advantages. Firstly, the same acoustic eigenproperties used in the previous chapters can be used to compute the Green's function needed to predict the pressure, i.e. same tire cavity system. Secondly, the screen effect at the coupling interfaces can be easily accounted for in the formulation. This second approach is implemented in this work to model the control technique. 
For simplicity and without loss of generality, the secondary cavities are modeled as a constant cross section straight waveguide (tube) as shown in Figures 4.2 and 4.3. The size of the tube cross section is assumed much smaller than the acoustic wavelength. Since for typical tires the acoustic resonance $(0,1,0)$ is at few hundred Hertz, this assumption is valid and implies that the motion of the boundary at the coupling interfaces can be modeled as a piston sources with unknown normal velocity $\dot{z}_{j}$ with $j=1,2$.

The couple response of the tire structure and acoustic cavity (tire and secondary cavities) is again the solution of the system

$$
\left(\left[L_{c}\right]-\Omega^{2}[I]\right)\left\{\begin{array}{l}
u \\
v \\
w
\end{array}\right\}=\left\{\begin{array}{l}
f_{u} \\
f_{v} \\
f_{w}
\end{array}\right\}-\left\{\begin{array}{c}
0 \\
0 \\
p(x, \theta, a)
\end{array}\right\}
$$

where the expression for the pressure $p(x, \theta, a)$ has to now include the effect of the secondary cavities, i.e. piston sources. To this end, the acoustic pressure in the tire main cavity is given as

$$
\begin{aligned}
p(x, \theta, a)= & \int_{0}^{L_{T}} \int_{0}^{2 \pi} i \omega \rho \dot{w}\left(x_{o}, \theta_{o}\right) G\left(x, \theta, a \mid x_{o}, \theta_{o}, a\right) a \mathrm{~d} \theta_{o} d x_{o} \\
& -\sum_{j=1}^{2} \int_{0}^{\gamma} \int_{0}^{r_{j}} i \omega \rho \dot{z}_{j} G\left(x, \theta, a \mid x_{o}^{(j)}, \theta_{o}^{(j)}, b\right) \bar{r} \mathrm{~d} \bar{r} d \gamma
\end{aligned}
$$

It can be observed that the first term in this equation is the pressure produced by the elastic shell motion while the second term accounts for the effect of the motion of the piston sources at the coupling interfaces. 

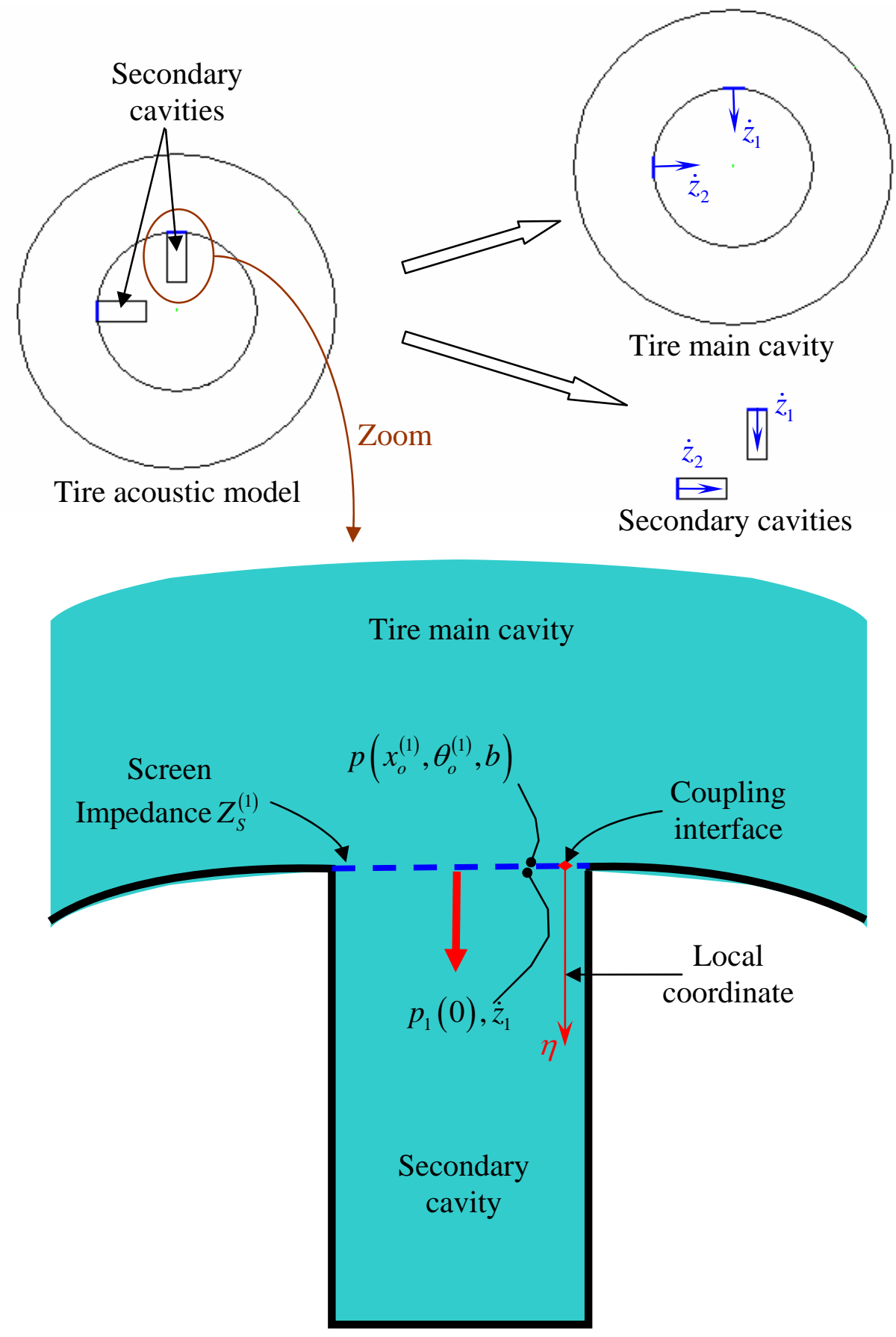

Figure 4.3: Schematic of the acoustic model and coupling interface. 
From the assumption of the acoustic wavelength much larger than the size of the secondary cavity cross section, equation (4.2) becomes

$$
\begin{aligned}
p(x, \theta, a)= & \int_{0}^{L_{T}} \int_{0}^{2 \pi} i \omega \rho \dot{w}\left(x_{o}, \theta_{o}\right) G\left(x, \theta, a \mid x_{o}, \theta_{o}, a\right) a \mathrm{~d} \theta_{o} d x_{o} \\
& -\sum_{j=1}^{2} S_{j} G\left(x, \theta, a \mid x_{o}^{(j)}, \theta_{o}^{(j)}, b\right) i \omega \rho \dot{z}_{j}
\end{aligned}
$$

where $S_{j}$ is the cross sectional area of the $j^{\text {th }}$ secondary cavities. The Green's function $G\left(x, \theta, r \mid x_{o}^{(j)}, \theta_{o}^{(j)}, b\right)$ is the same one obtained in Chapter 3, i.e. for the uniform torus tire cavity, where the source is at the center of the coupling interface with coordinate $\left(x_{o}^{(j)}, \theta_{o}^{(j)}, b\right)$.

On the other hand, the acoustic pressure in the secondary cavity can also be expressed in terms of the piston motion as

$$
p_{j}(\eta)=G_{j}(\eta \mid 0) i \omega \rho \dot{z}_{j} S_{j}
$$

where the Green's function for the waveguide secondary cavity is easily obtained as (Kinsler et. al., 2000)

$$
G_{j}\left(\eta \mid \eta_{0}\right)=\sum_{i=0}^{N A} \frac{\cos \left(k_{i}^{(j)} \eta\right) \cos \left(k_{i}^{(j)} \eta_{o}\right)}{\varepsilon_{i} S_{j} L_{j}\left(\left(k_{i}^{(j)}\right)^{2}-k^{2}\right)}
$$

where $L_{j}$ is the cavity length, $k_{i}^{(j)}=l \pi / L_{j}$ is the axial waveguide wavenumber, and $\eta$ is the local coordinate system in the secondary cavity as shown in Figure 4.3. 
In order to find the piston velocity $\dot{z}_{j}$, the continuity of acoustic pressure at the coupling interface must be satisfied as illustrated in Figure 4.3. Note that continuity of particle velocity is automatically satisfied since the same piston velocity is used in both cavities, i.e. equations (4.3) and (4.4). The continuity of pressure is defined as Hallez et. al. (2000)

$$
p\left(x_{o}^{(j)}, \theta_{o}^{(j)}, b\right)=p_{j}(0)+Z_{S}^{(j)} \dot{z}_{j} \quad j=1,2, \ldots
$$

where $Z_{S}^{(j)}$ is accounting for the acoustic impedance of the screen installed at the coupling interface. An example of the screen impedance for a perforated plate is presented in Appendix F.

For the case of the two secondary cavities considered here, replacing equations (4.4) and (4.3) into the system of equation given by (4.6), the equations for the piston velocities $\dot{z}_{1}$ and $\dot{z}_{2}$ becomes

$$
\left[\begin{array}{ll}
z_{11} & z_{12} \\
z_{21} & z_{22}
\end{array}\right]\left\{\begin{array}{l}
\dot{z}_{1} \\
\dot{z}_{2}
\end{array}\right\}=\left\{\begin{array}{l}
\int_{0}^{L_{T}} \int_{0}^{2 \pi} i \omega \rho \dot{w}\left(x_{o}, \theta_{o}\right) G\left(x_{o}^{(1)}, \theta_{o}^{(1)}, b \mid x_{o}, \theta_{o}, a\right) a \mathrm{~d} \theta_{o} d x_{o} \\
\int_{0}^{L_{T}} \int_{0}^{2 \pi} i \omega \rho \dot{w}\left(x_{o}, \theta_{o}\right) G\left(x_{o}^{(2)}, \theta_{o}^{(2)}, b \mid x_{o}, \theta_{o}, a\right) a \mathrm{~d} \theta_{o} d x_{o}
\end{array}\right\}
$$

where

$$
\begin{aligned}
& z_{11}=i \omega \rho S_{1} G_{1}(0 \mid 0)+Z_{S}^{(1)}+i \omega \rho S_{1} G\left(x_{o}^{(1)}, \theta_{o}^{(1)}, b \mid x_{o}^{(1)}, \theta_{o}^{(1)}, a\right) \\
& z_{22}=i \omega \rho S_{2} G_{2}(0 \mid 0)+Z_{S}^{(2)}+i \omega \rho S_{2} G\left(x_{o}^{(2)}, \theta_{o}^{(2)}, b \mid x_{o}^{(2)}, \theta_{o}^{(2)}, a\right) \\
& z_{12}=i \omega \rho S_{2} G\left(x_{o}^{(1)}, \theta_{o}^{(1)}, b \mid x_{o}^{(2)}, \theta_{o}^{(2)}, b\right) \\
& z_{21}=i \omega \rho S_{1} G\left(x_{o}^{(2)}, \theta_{o}^{(2)}, b \mid x_{o}^{(1)}, \theta_{o}^{(1)}, b\right)
\end{aligned}
$$

The unknown piston velocities $\dot{z}_{1}$ and $\dot{z}_{2}$ are solve explicitly as 
Leonardo Molisani Chapter 4: Control of the Tire Acoustic

$$
\begin{gathered}
\dot{z}_{1}=\frac{1}{z_{11} z_{22}-z_{12} z_{21}}\left\{z_{22}\left[\int_{0}^{L_{T}} \int_{0}^{2 \pi} \dot{w}\left(x_{o}, \theta_{o}\right) G\left(x_{o}^{(1)}, \theta_{o}^{(1)}, b \mid x_{o}, \theta_{o}, a\right) a d \theta_{o} d x_{o}\right]\right. \\
\left.-z_{12}\left[\int_{0}^{L_{T}} \int_{0}^{2 \pi} \dot{w}\left(x_{o}, \theta_{o}\right) G\left(x_{o}^{(2)}, \theta_{o}^{(2)}, b \mid x_{o}, \theta_{o}, a\right) a d \theta_{o} d x_{o}\right]\right\}
\end{gathered}
$$

and

$$
\begin{gathered}
\dot{z}_{2}=\frac{1}{z_{11} z_{22}-z_{12} z_{21}}\left\{z_{11}\left[\int_{0}^{L_{T}} \int_{0}^{2 \pi} \dot{w}\left(x_{o}, \theta_{o}\right) G\left(x_{o}^{(2)}, \theta_{o}^{(2)}, b \mid x_{o}, \theta_{o}, a\right) a d \theta_{o} d x_{o}\right]\right. \\
\left.-z_{21}\left[\int_{0}^{L} \int_{0}^{2 \pi} \dot{w}\left(x_{o}, \theta_{o}\right) G\left(x_{o}^{(1)}, \theta_{o}^{(1)}, b \mid x_{o}, \theta_{o}, a\right) a d \theta_{o} d x_{o}\right]\right\}
\end{gathered}
$$

Replacing equations (4.9) and (4.10) into equation (4.2), the acoustic pressure in the tire main cavity becomes,

$$
p(x, \theta, a)=\int_{0}^{L_{T}} \int_{0}^{2 \pi} \hat{G}\left[x, \theta, a \mid\left(x_{o}, x_{o}^{(1)}, x_{o}^{(2)}\right),\left(\theta_{o}, \theta_{o}^{(1)}, \theta_{o}^{(2)}\right),(a, b, b)\right] i \omega \rho \dot{w}\left(x_{o}, \theta_{o}\right) a \mathrm{~d} \theta_{o} d x_{o}
$$

where

$$
\begin{aligned}
\hat{G}\left[x, \theta, a \mid\left(x_{o}, x_{o}^{(1)}, x_{o}^{(2)}\right),\left(\theta_{o}, \theta_{o}^{(1)}, \theta_{o}^{(2)}\right),(a, b, b)\right]=G\left(x, \theta, a \mid x_{o}, \theta_{o}, a\right)+\frac{1}{z_{11} z_{22}-z_{12} z_{21}} \\
\left\{G\left(x_{o}^{(1)}, \theta_{o}^{(1)}, b \mid x_{o}, \theta_{o}, a\right)\left[S_{2} G\left(x, \theta, r \mid x_{o}^{(2)}, \theta_{o}^{(2)}, b\right) z_{21}-S_{1} G\left(x, \theta, r \mid x_{o}^{(1)}, \theta_{o}^{(1)}, b\right) z_{22}\right]\right. \\
\left.+G\left(x_{o}^{(2)}, \theta_{o}^{(2)}, b \mid x_{o}, \theta_{o}, a\right)\left[S_{1} G\left(x, \theta, r \mid x_{o}^{(1)}, \theta_{o}^{(1)}, b\right) z_{12}-S_{2} G\left(x, \theta, r \mid x_{o}^{(2)}, \theta_{o}^{(2)}, b\right) z_{11}\right]\right\}
\end{aligned}
$$

represents the “modified” Green's function accounting for the effect of the secondary cavities on the tire cavity.

Equation (4.11) is then substituted into the equation of motion (4.1) to account for the acoustic pressure loading. Following the same steps as in chapter 3, the modal amplitudes of the tire (fully coupled to tire and secondary cavities) can be written as 
$\left[\left[K_{s r}\right]-\Omega^{2}\left[\left[M_{s r}\right]-\sum_{q=0}^{Q} \sum_{p=0}^{P} \sum_{l=0}^{L}\left[\alpha_{\text {srmnqpl }}\right]-\sum_{q=0}^{Q} \sum_{p=0}^{P} \sum_{l=0}^{L}\left[\alpha_{\text {srmnqpl }}^{(1)}\right]-\sum_{q=0}^{Q} \sum_{p=0}^{P} \sum_{l=0}^{L}\left[\alpha_{\text {srmnqpl }}^{(2)}\right]\right]\right]\left\{A_{s r}\right\}=\left\{f_{s r}\right\}$

Equation (4.13) is a coupled algebraic system of equations that must be solved simultaneously to obtain the unknown modal amplitudes. These modal amplitudes can then be used to compute the force and moment at the spindle. The computation of $\sum_{q=0}^{Q} \sum_{p=0}^{P} \sum_{l=0}^{L}\left[\alpha_{\text {srmnqpl }}\right], \sum_{q=0}^{Q} \sum_{p=0}^{P} \sum_{l=0}^{L}\left[\alpha_{\text {srmnqpl }}^{(1)}\right]$, and $\sum_{q=0}^{Q} \sum_{p=0}^{P} \sum_{l=0}^{L}\left[\alpha_{\text {srmnqpl }}^{(2)}\right]$ is given in Appendix G.

The approach presented in this section can be easily extended to any arbitrary shaped added second cavity geometry. The procedure requires only knowing the spectral properties of the secondary cavity.

\subsection{NUMERICAL SIMULATIONS OF SECONDARY CAVITIES CONTROL APPROACH}

A numerical evaluation for the free and deformed tire models with the proposed secondary cavities control approach is presented in this section. The tire size used in the analysis is again the 195/65 R15 with the same material properties used in Chapter 2. The main goal of this section is to determine the effectiveness of this approach to control the tire cavity resonance problem. Another goal is to investigate the effect of the secondary cavity properties such as length, cross sectional area, and screen impedance on the performance.

In order to define the length of the secondary cavities, the waveguide cavity axial resonance must be tuned to the tire cavity resonance at $232.6 \mathrm{~Hz}$ (see Table 3.3). The tuning of the cavity leads to the length of the secondary cavities to be a quarter of the wavelength associated to the tire main cavity resonance, Beranek and Vér (1992). The wavelength of the tire main cavity for the tire size 195/65 R15 is $\mathbf{1 . 4 7 5} \mathbf{~ m}$, i.e. 
approximately the tire centerline circumference. The secondary cavity resonances can be estimated assuming “close-open” boundary conditions, i.e. quarter wave tube. That is

$$
f_{i}=\frac{c}{4} \frac{(2 i+1)}{L_{j}} \quad i=0,1,2, \ldots
$$

where $L_{j}$ is the length of the secondary cavity. Simulations for different secondary cavity length are shown in Figures 4.4 and 4.5 for the spindle vertical force and the horizontal moment for the free tire model. In these simulation, the radius of the secondary cavity cross section was assumed to be $\mathbf{0 . 0 2} \boldsymbol{m}$ and as an initial value to set the length of the secondary cavity, the screen impedance was assumed purely resistive (only damping) with a value of $\mathbf{0 . 0 2} \boldsymbol{\rho}$ c. Later in the analysis to study the effect of the acoustic damping, a realistic model of the frequency dependant acoustic screen impedance for a perforated plate screen is used at the coupling interface (See Appendix F). From the results in these figures, it can be observed that the concept is very effective at suppressing the acoustic cavity resonance. The optimum secondary cavity length corresponds to a value of $L_{j}=0.368 \mathrm{~m}$. 


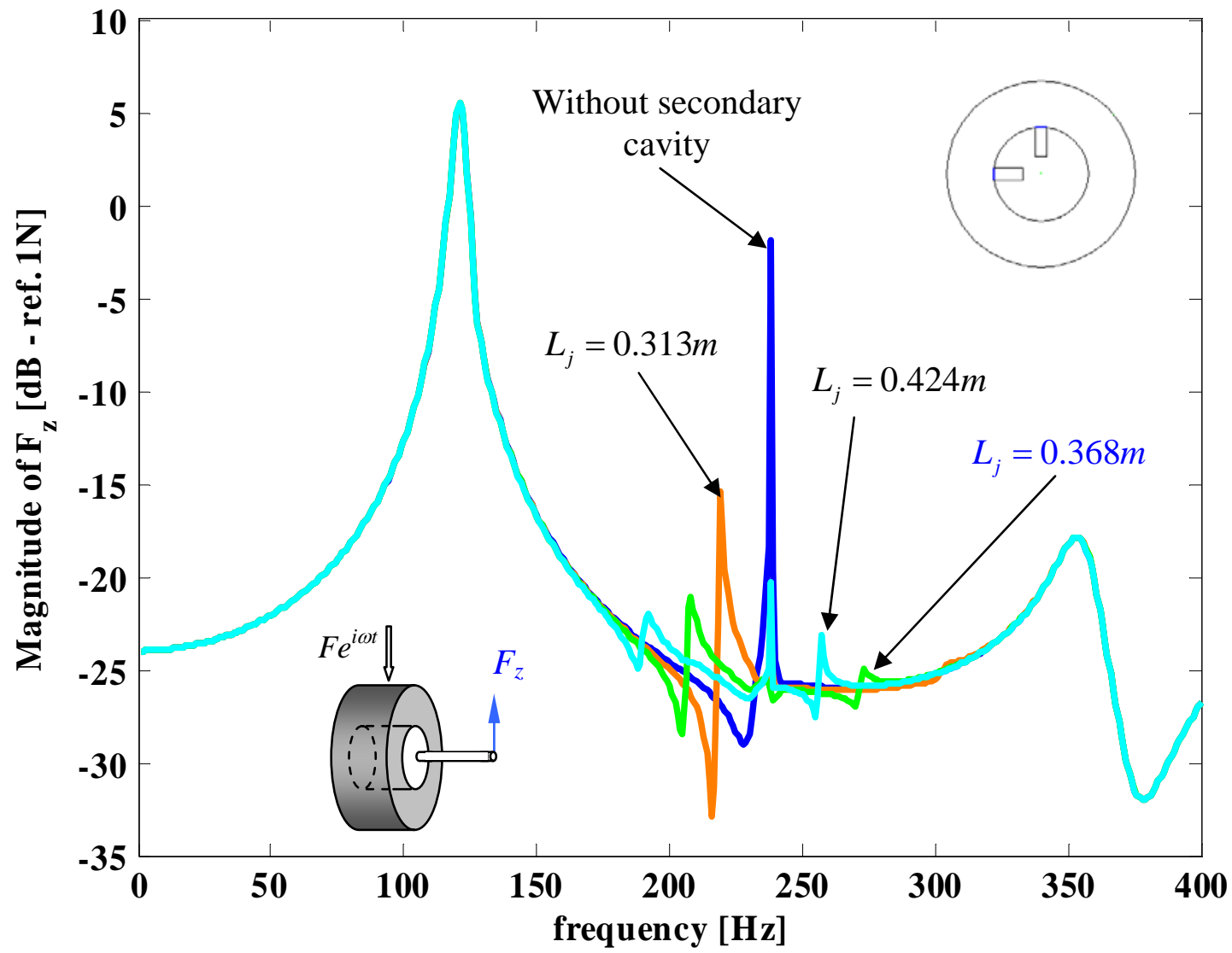

Figure 4.4: Magnitude of the vertical force at the spindle for different secondary cavity length for the free tire model. 


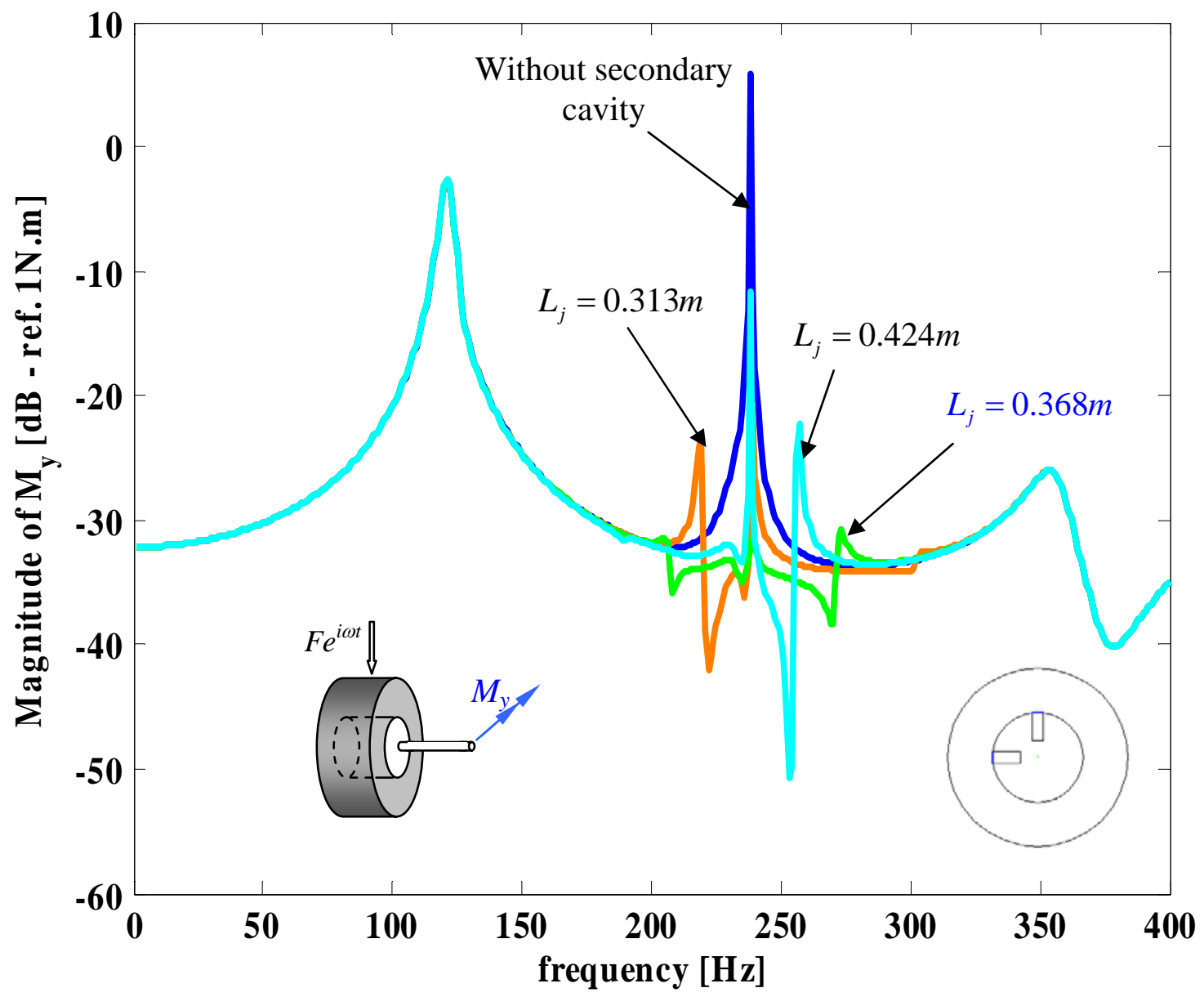

Figure 4.5: Magnitude of the horizontal moment at the spindle for different secondary cavity length for the free tire model.

The cross sectional area of the secondary cavities is the second parameter to be determined in this study. To this end, Figures 4.6 and 4.7 show the resultant force and moment at the spindle for different cross section radii. The increasing in the radius of the section the larger is the split of the main cavity resonance. The effect in the split is analogue to the effect when more mass is added in the secondary mass of the DVA. The results indicate that even a small cross sectional area will be effective at attenuating the cavity problem. 


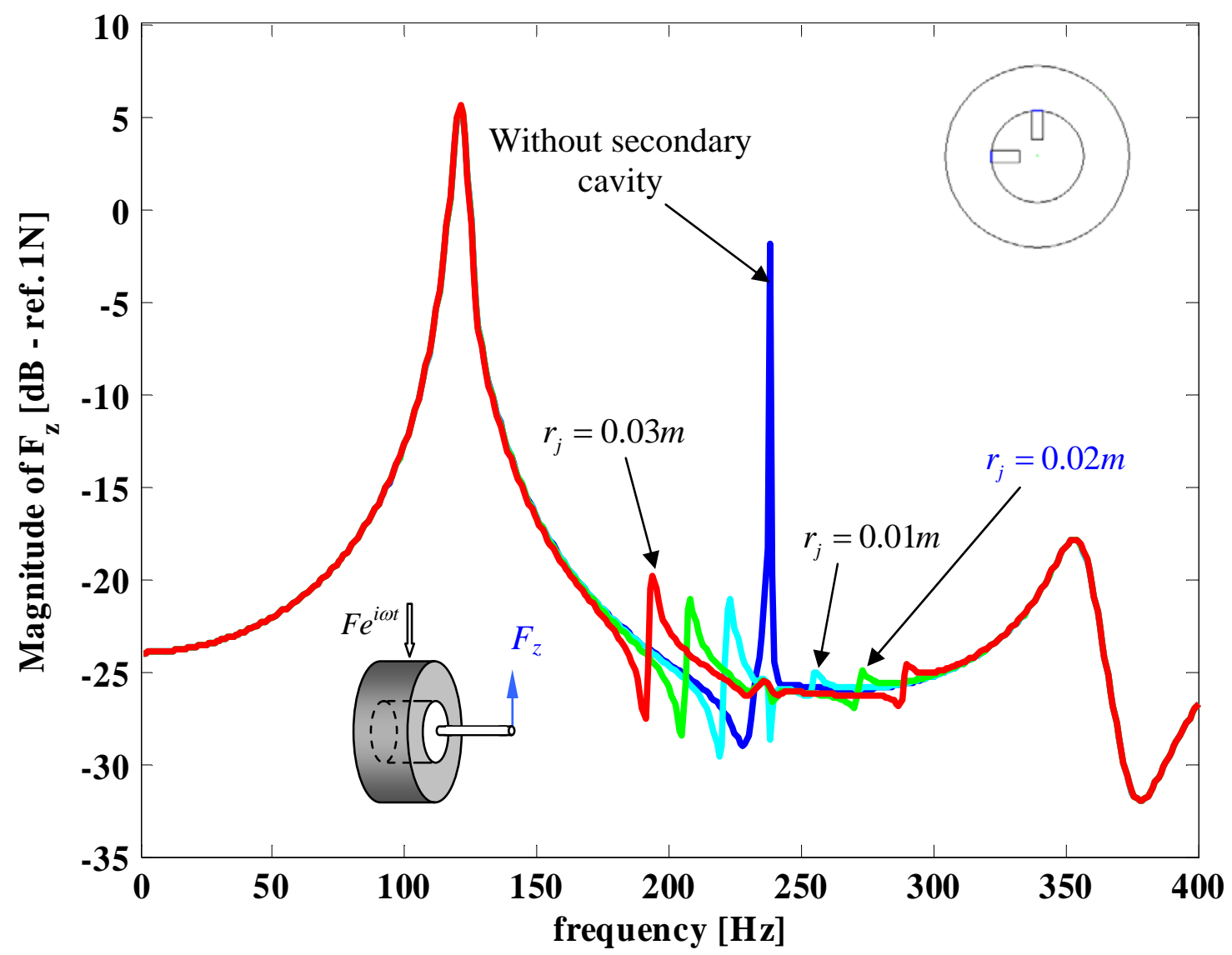

Figure 4.6: Magnitude of the vertical force at the spindle for different secondary cavity radius for the free tire model. 


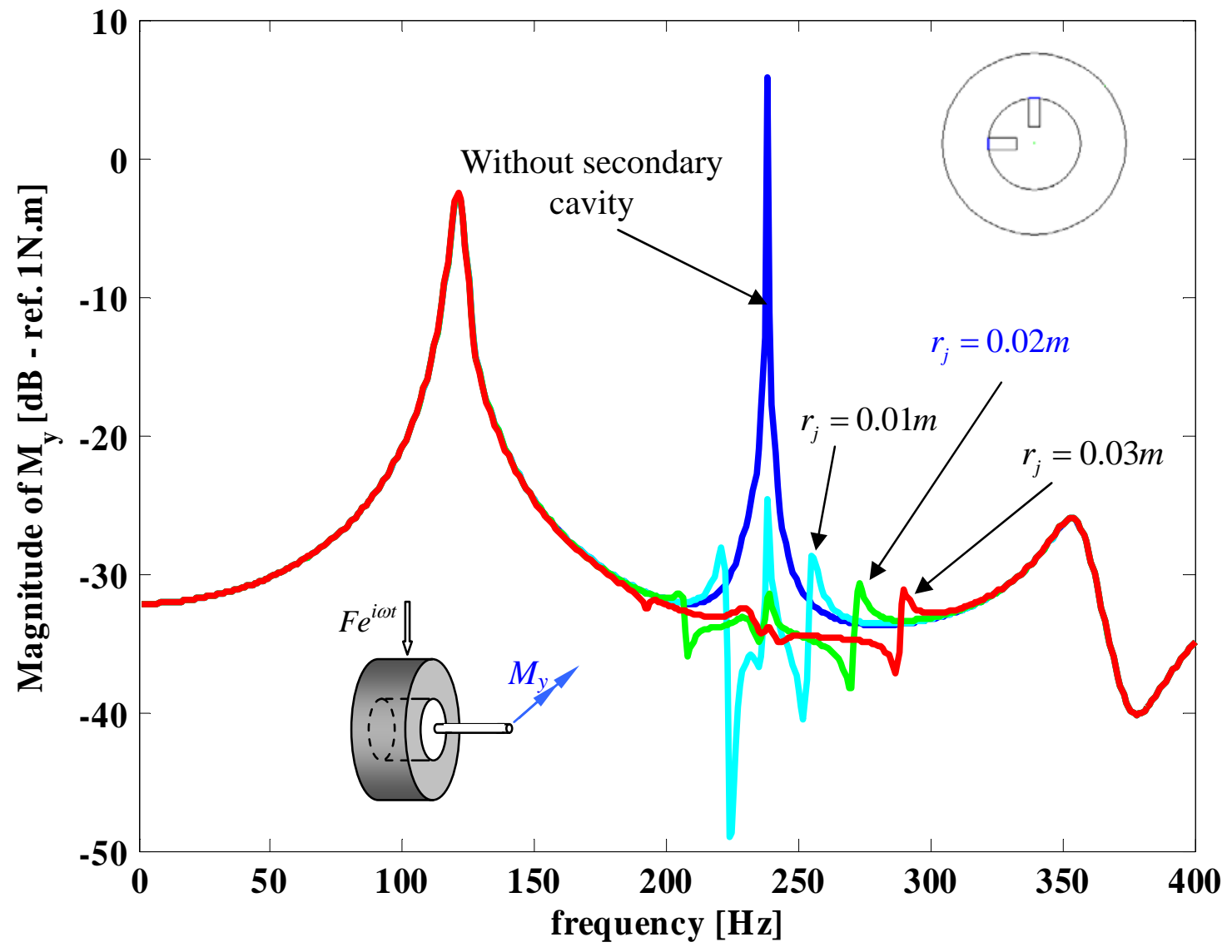

Figure 4.7: Magnitude of the horizontal moment at the spindle for different secondary cavity radius for the free tire model. 
The next study is the sensitivity study of the screen's impedance by using the model of a perforated plate presented in the Appendix F. The model of the impedance [units: $N \frac{\mathrm{S}}{\mathrm{m}^{3}}$ ] is mainly a function of the thickness of the perforated screen, the radius of the orifices at the screen, and the percentage of open area (POA) due to the screen porosity. The fixed screen parameters were the screen thickness $(\mathbf{0 . 0 0 0 7 5 m})$ and the orifices radius $(\mathbf{0 . 0 0 0 7 5 m})$. The screen POA was investigated for the cases $\mathbf{1 5 \%}, \mathbf{2 0 \%}$, and $\mathbf{2 5 \%}$. The length and cross section radius of the secondary cavities were also fixed at the values of $0.368 \mathrm{~m}$ and $0.02 \mathrm{~m}$, respectively. Figures 4.8 and 4.9 show the resultant force and moment at the spindle for the different POA values. The results show that the attenuation is basically insensitive for the range of POAs evaluated. From the analysis, a screen resistance of $20 \%$ of $P O A$ is selected.

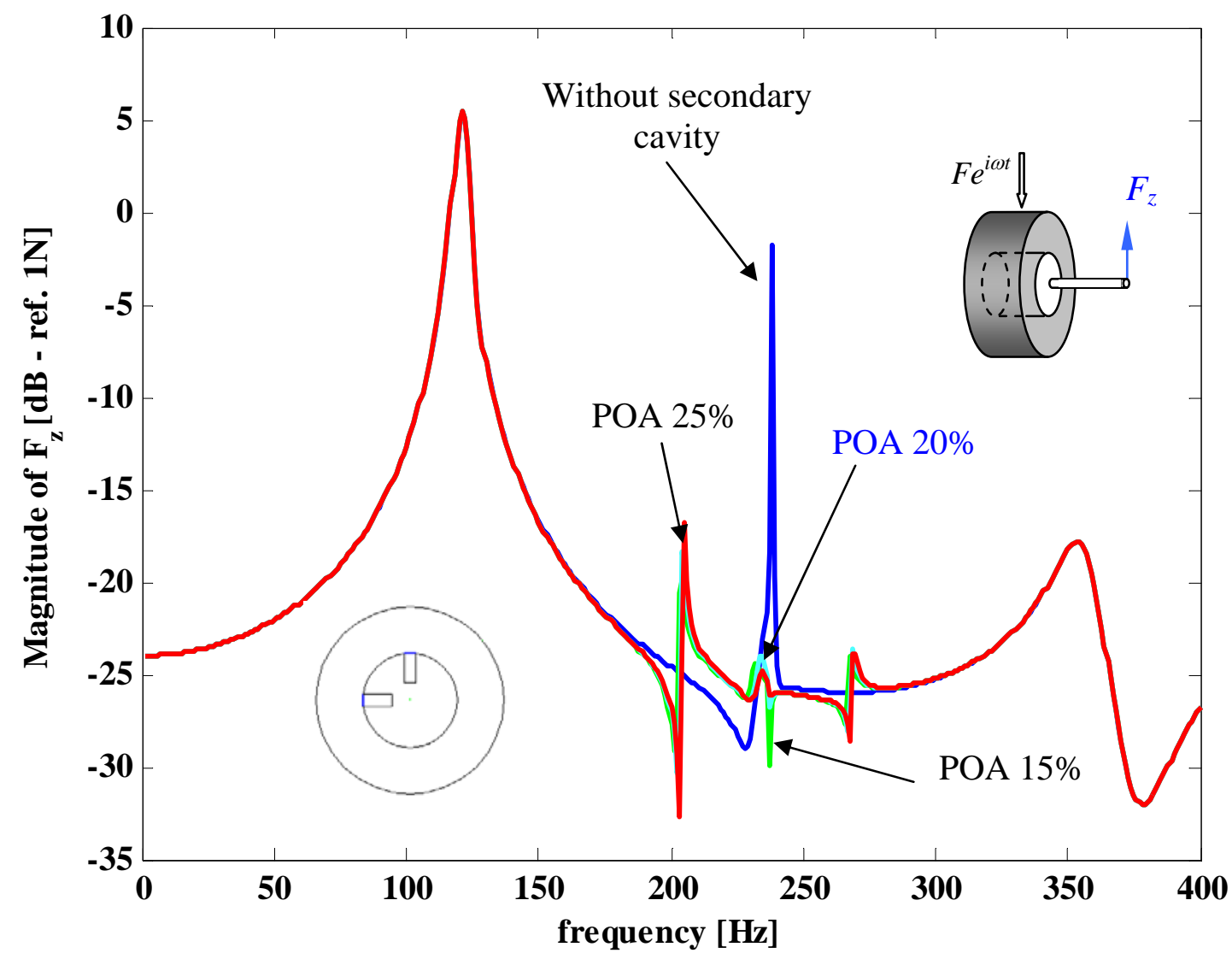

Figure 4.8: Magnitude of the vertical force at the spindle for different screen impedance for the free tire model. 


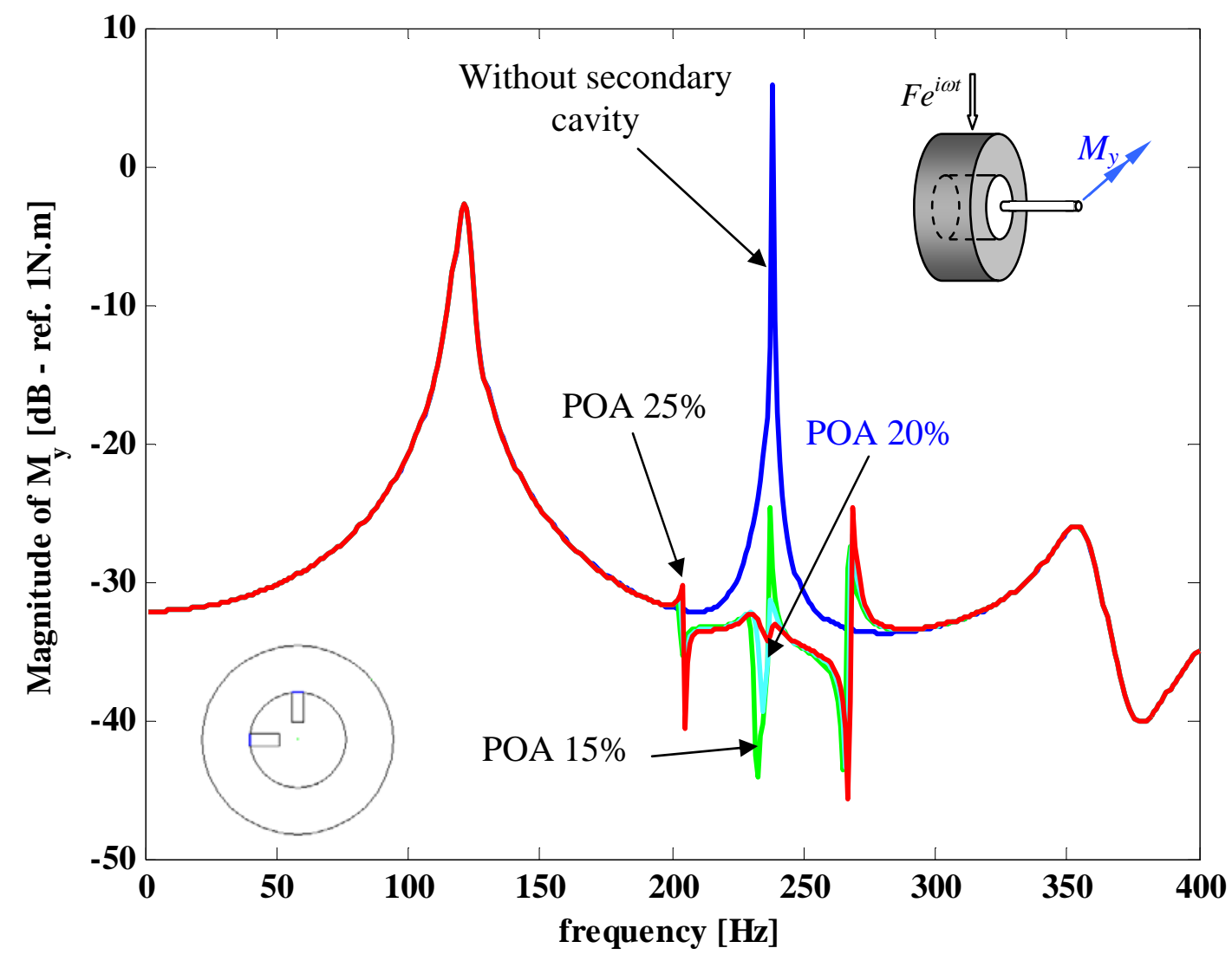

Figure 4.9: Magnitude of the horizontal moment at the spindle for different screen impedance for the free tire model. 
For the sake of completeness, the predicted magnitude of the point accelerance is shown in Figure 4.10 for the in vacuo model (red dotted line), the coupled model with the secondary cavities (solid blue line), and the measurement (green line from Yamauchi and Akiyoshi, 2002). The secondary cavities with the selected parameters successfully achieve the resonance control as seen in the figure.

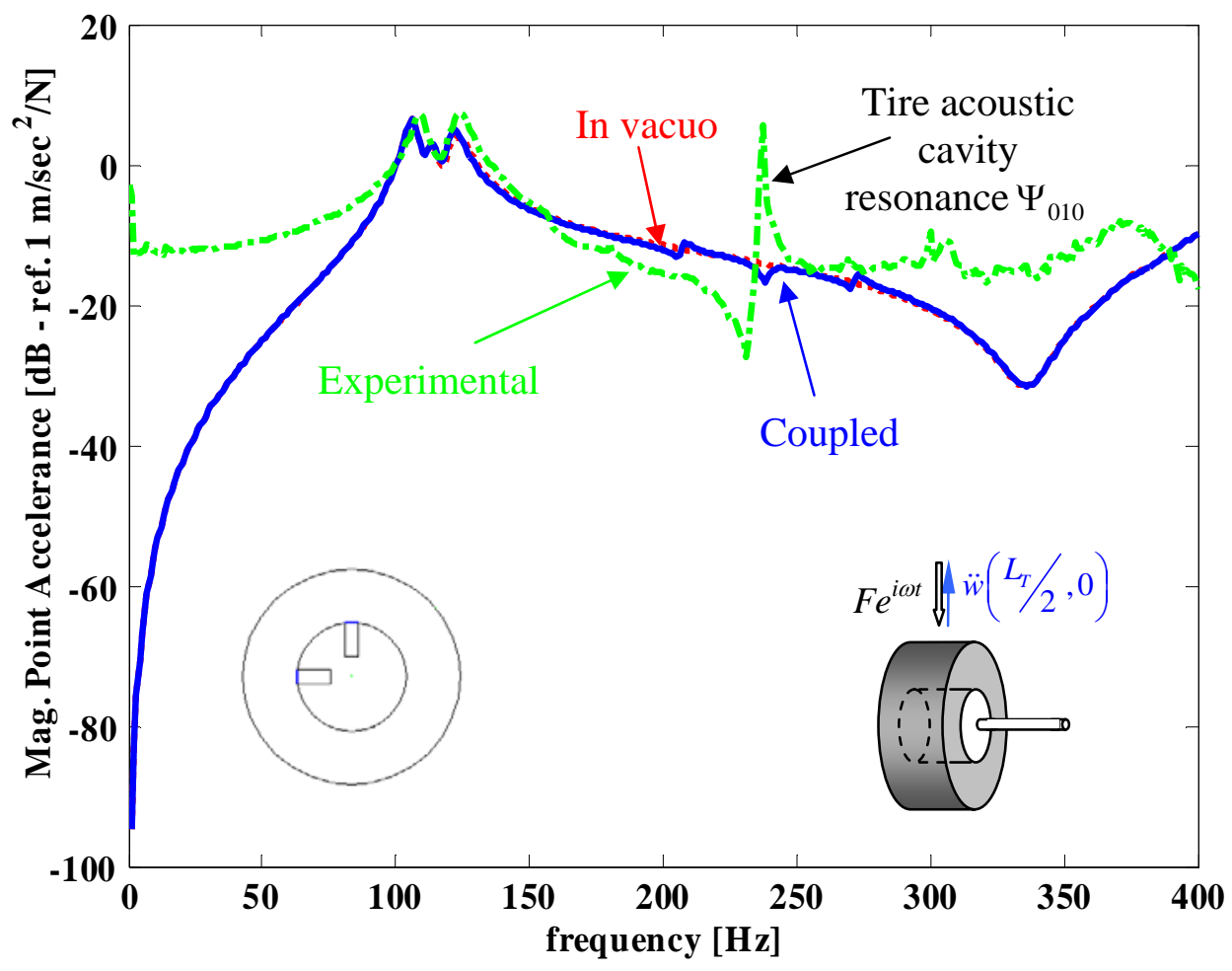

Figure 4.10: Magnitude of the point accelerance for the free tire model.

The numerical simulation studies performed above assumed that one of the secondary cavities was positioned at $\theta_{o}^{(1)}=0^{\circ}$, i.e. at the anti-node of the $(0,1,0)$ mode. That is this secondary cavity was at the optimum position. Since the tire is rotating, the position of the secondary cavities with respect to the $(0,1,0)$ mode will be varying. The worst positing would be when neither secondary cavity is at the acoustic mode anti-node, i.e. $\theta_{o}^{(1)}=45^{\circ}$ and $\theta_{o}^{(2)}=135^{\circ}$. Note also that the vehicle traveling to a $55 \mathrm{mph}$ with tire size 195/65 R15 produce an angular variation of around $7 \mathrm{~Hz}$ while the secondary cavities 
resonate at $232.6 \mathrm{~Hz}$. Therefore, the rolling of the tire can be considered as a quasi-static effect. That is the rotation can be neglected and the analysis performed at fixed angular positions of the tire.

To quantify the performance of the control approach as the tire rotates, simulations were performed for the two cavities positioned at $\theta_{o}^{(1)}=45^{\circ}$ and $\theta_{o}^{(2)}=135^{\circ}$. The resultant force and moment at the spindle for the secondary cavity parameters $\boldsymbol{L}_{j}=\mathbf{0 . 3 6 8} \boldsymbol{m}$ and $\boldsymbol{r}_{\boldsymbol{j}}=\mathbf{0 . 0 2 \boldsymbol { m }}$ are shown in Figures 4.11 and 4.12, respectively. Comparing these results to the case in Figures 4.8 and 4.9 show that even in this most adverse position, the secondary cavities control approach is very effective.

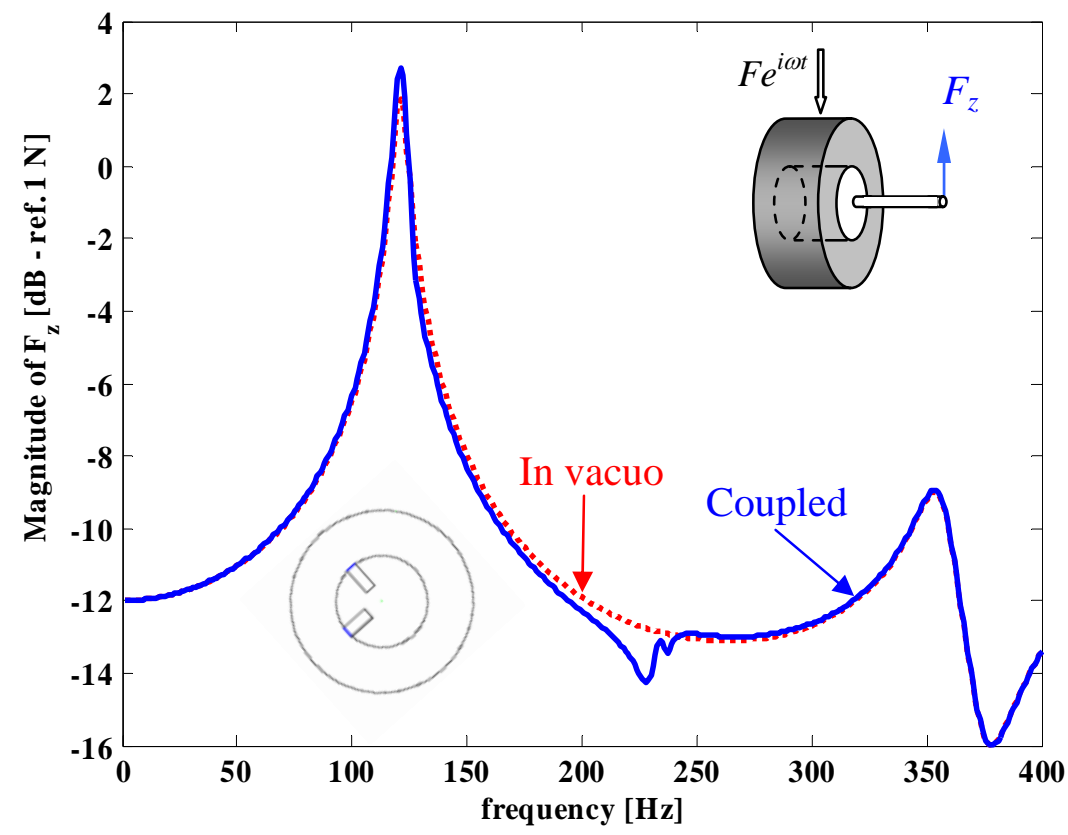

Figure 4.11: Magnitude of the vertical force at the spindle for different secondary cavity azimuth position for the free tire model. 


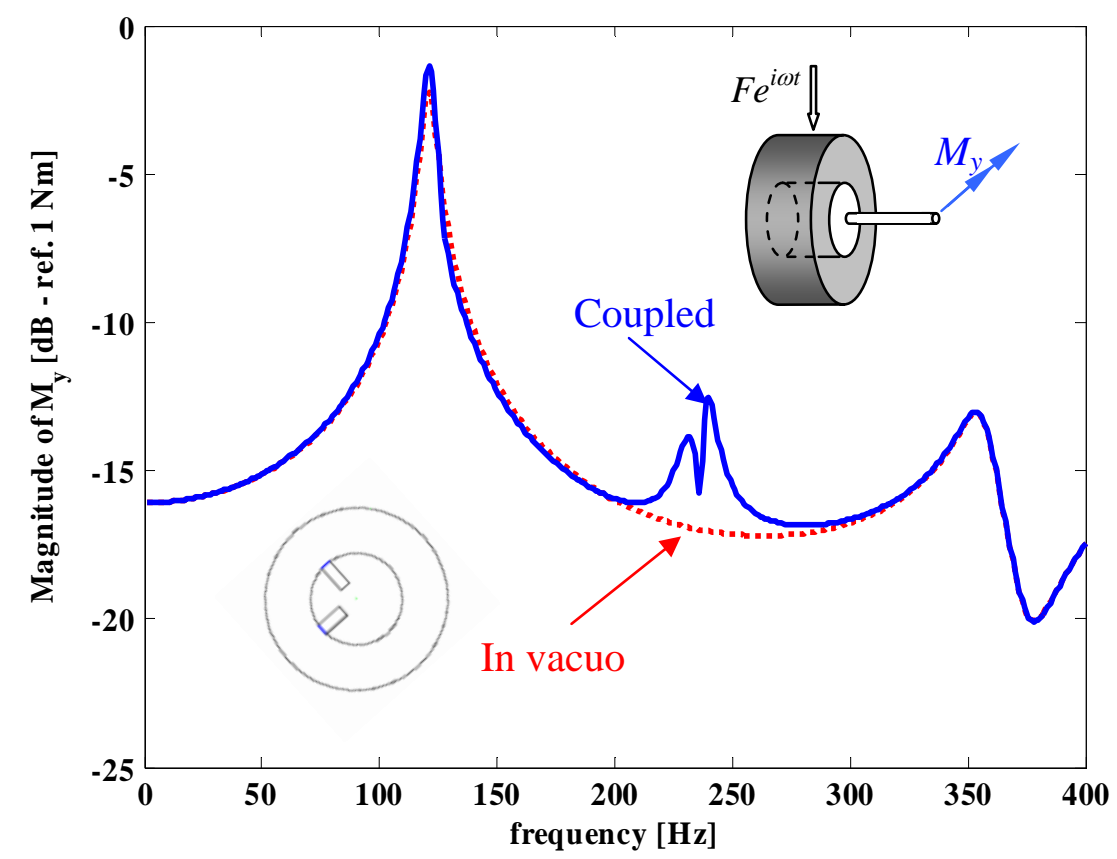

Figure 4.12: Magnitude of the horizontal moment at the spindle for different secondary cavity azimuth position for the free tire model.

In the case of the deformed tire, the length of the secondary cavities that achieved best results once again is $L_{j}=0.386 \mathrm{~m}$ as shown in Figures 4.13 and 4.14 for the vertical force and horizontal moment at the spindle. 


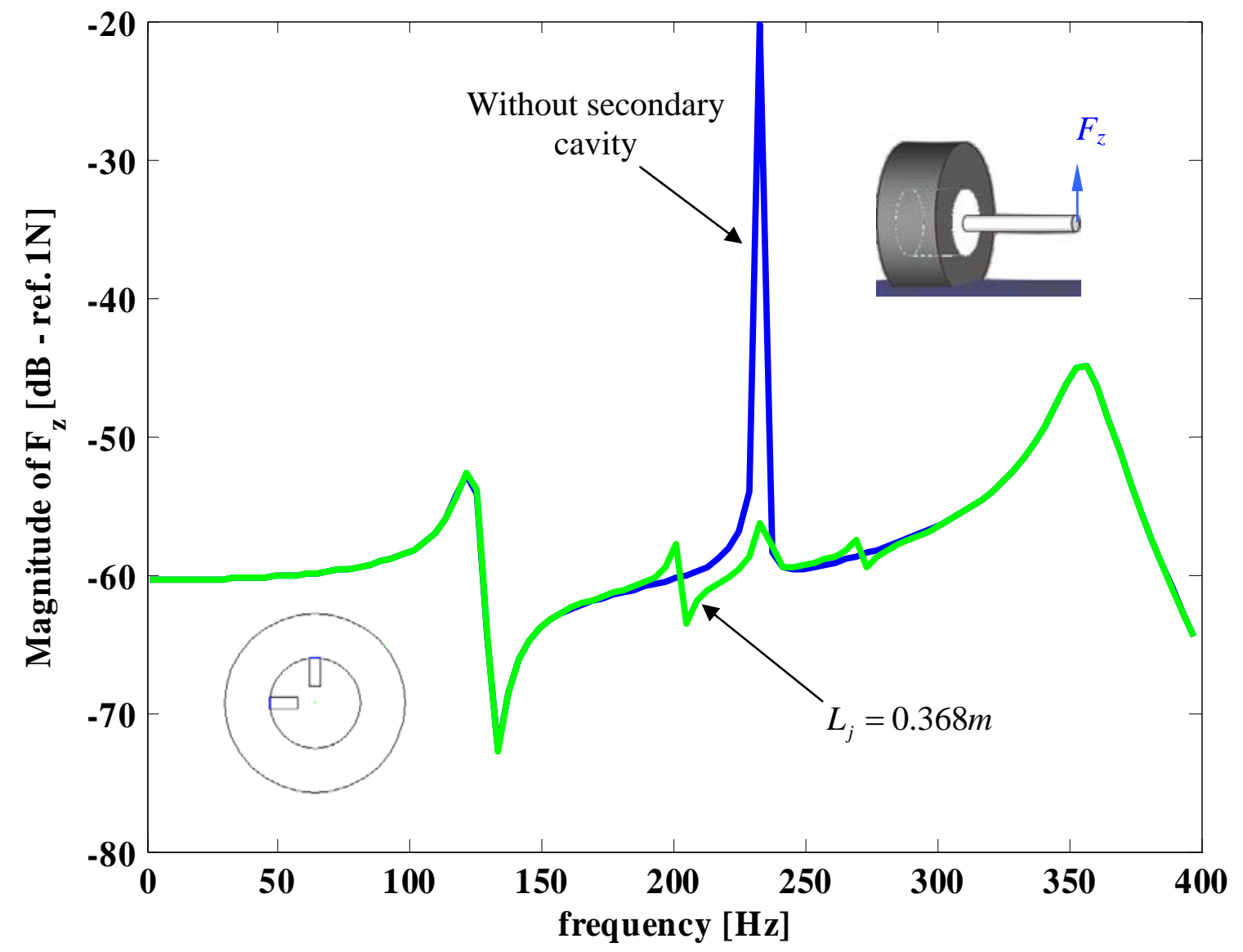

Figure 4.13: Magnitude of the vertical force at the spindle for the deformed tire model. 


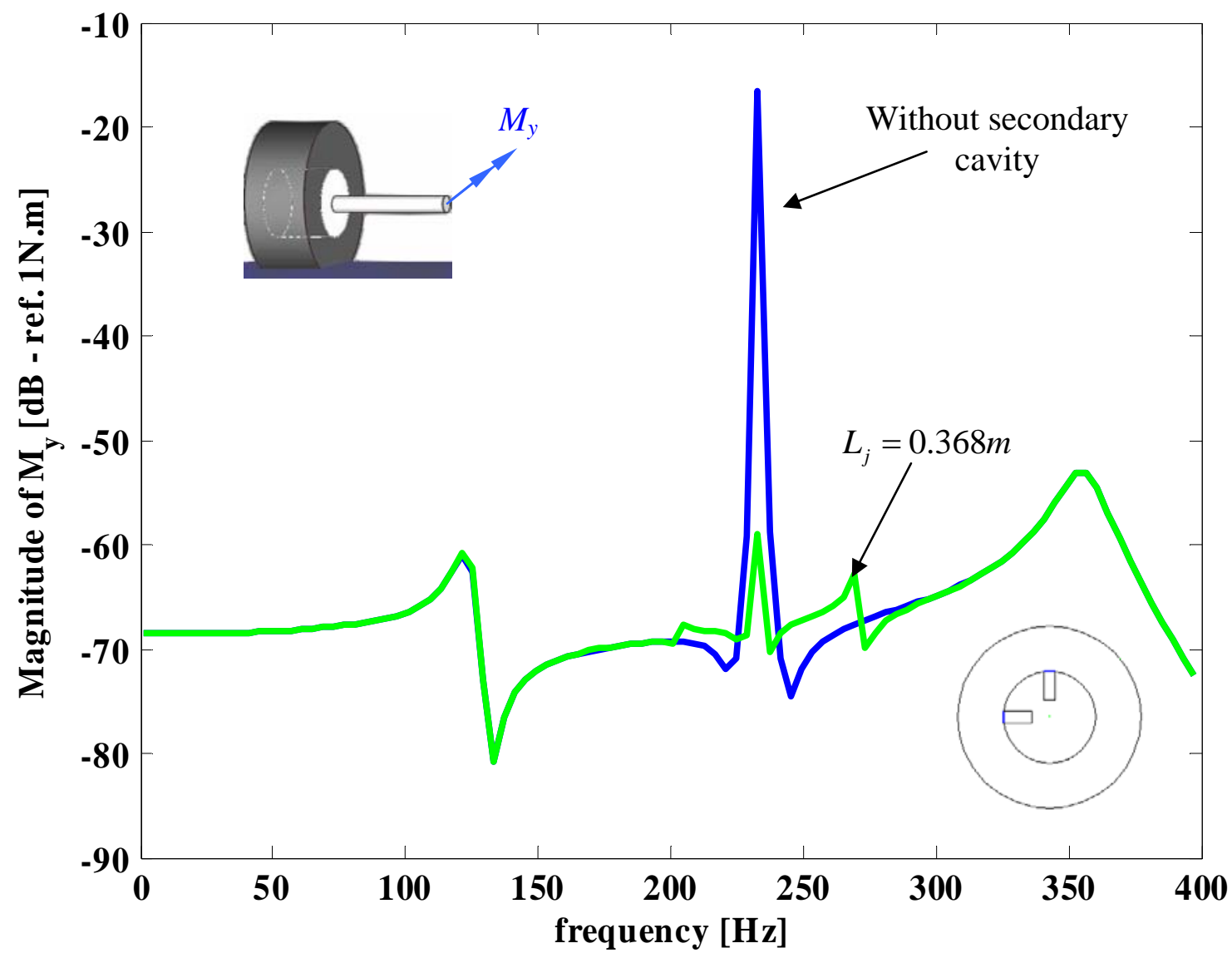

Figure 4.14: Magnitude of the horizontal moment at the spindle for the deformed tire model.

The cross section of the secondary cavities is also evaluated for the deformed tire. The radius of $0.02 \mathrm{~m}$ is chosen for the cross section. The effect of added mass can be seen in the Figures 4.15 and 4.16 for the main tire resonance split as the section becomes larger the split in frequency increases. 


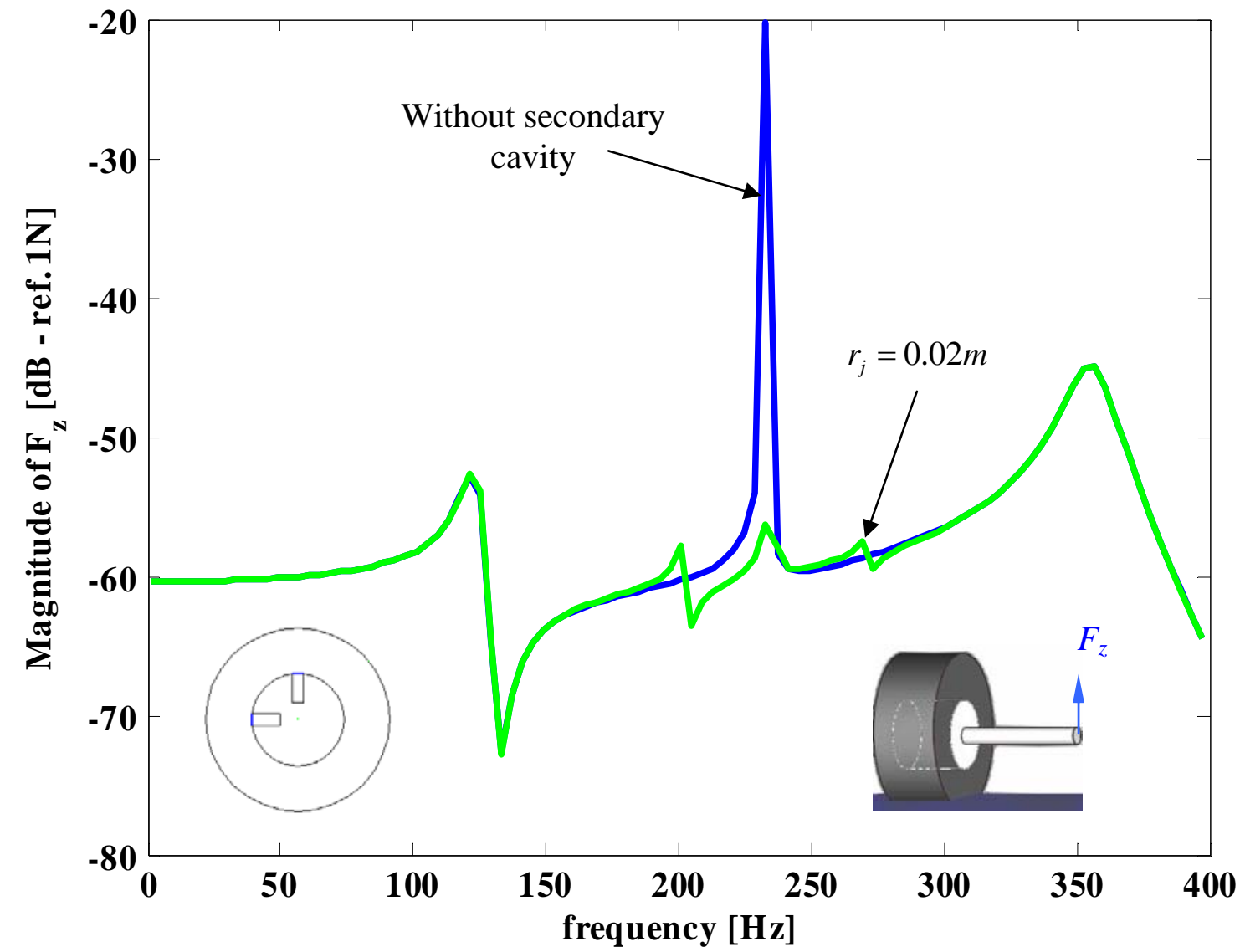

Figure 4.15: Magnitude of the vertical force at the spindle for the deformed tire model. 


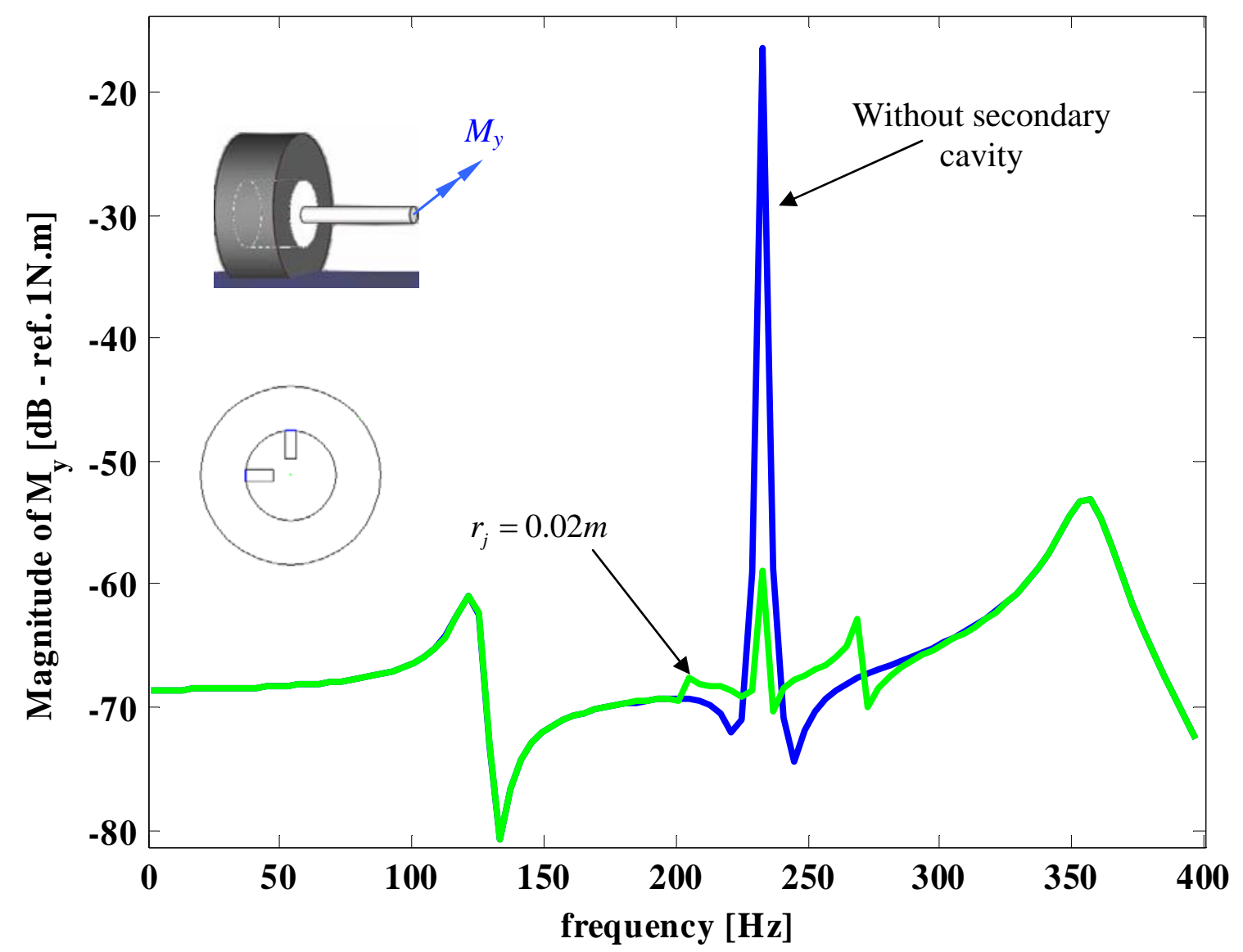

Figure 4.16: Magnitude of the horizontal moment at the spindle for the deformed tire model.

The resistance of screen impedance is evaluated for different cases for the force and moment. The results can be seen in Figures 4.17 and 4.18 respectively. A value of $\mathbf{2 0 \%}$ of $\boldsymbol{P O} \boldsymbol{A}$ is selected for the design of the acoustic system. 


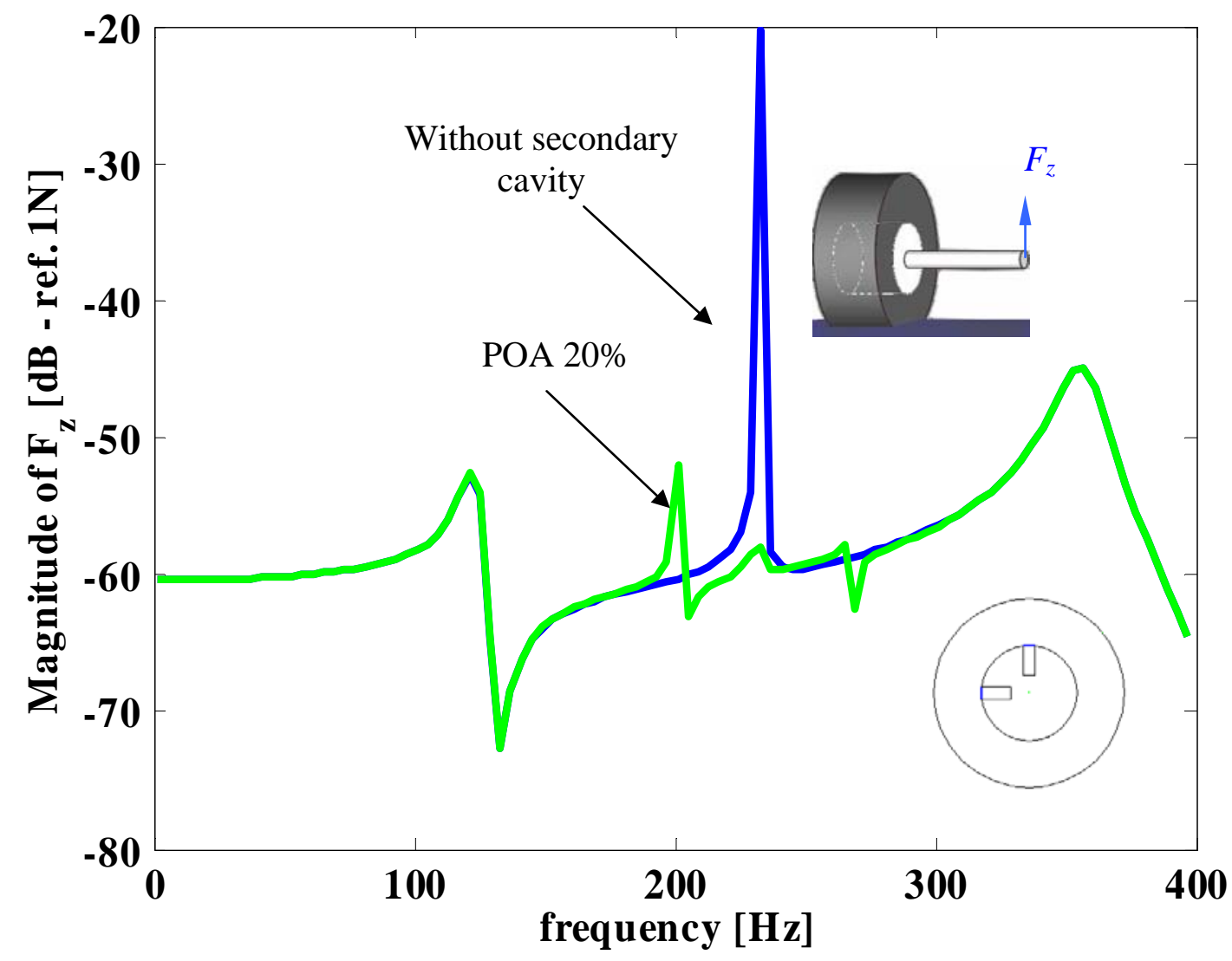

Figure 4.17: Magnitude of the vertical force at the spindle for the deformed tire model. 


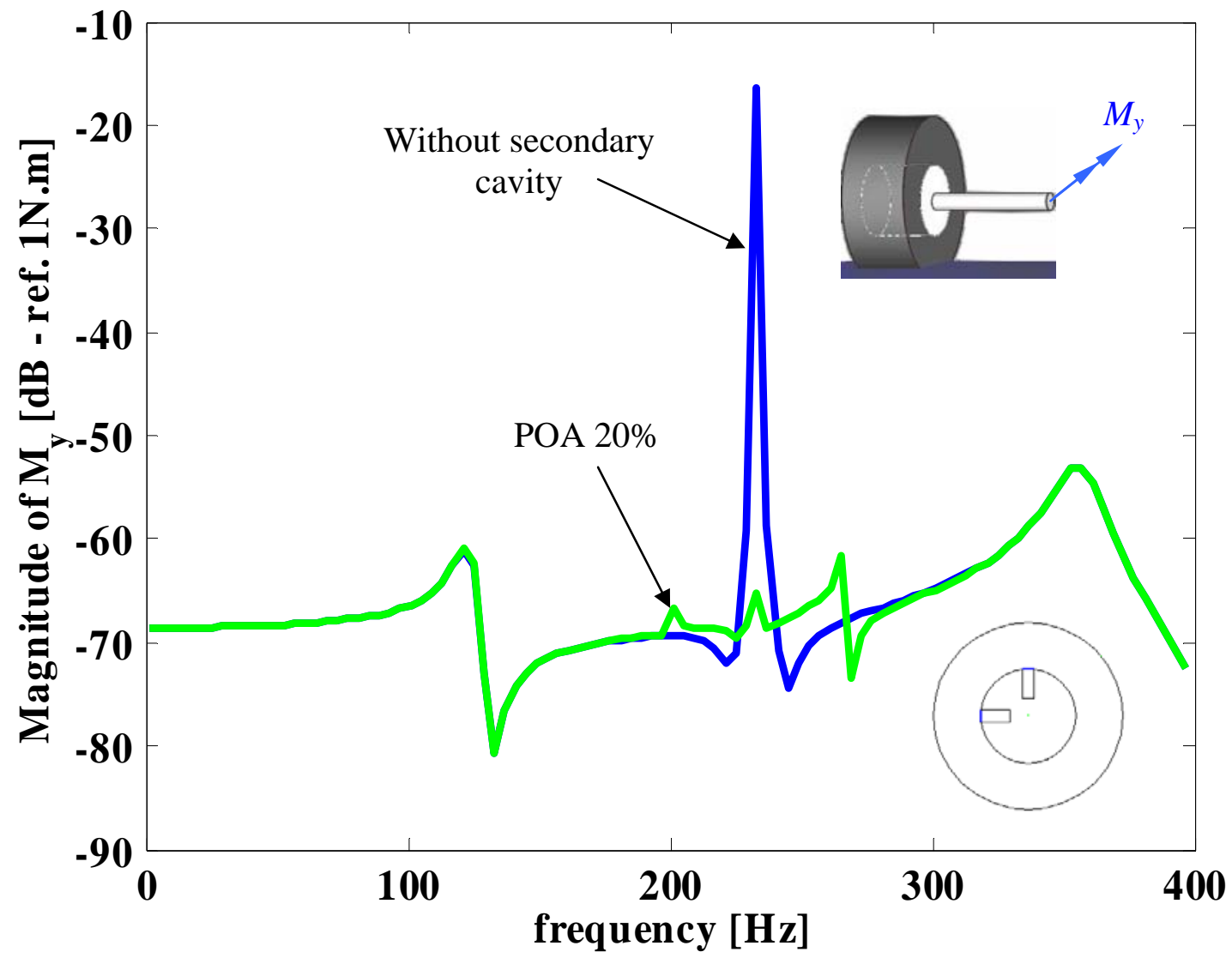

Figure 4.18: Magnitude of the horizontal moment at the spindle for the deformed tire model.

Figure 4.19 shows a comparison between the point accelerance for the in vacuo and coupled deformed tire model, and experimental measurement. In the figure, the tire cavity resonance shows no effect in the accelerance of the tire. 


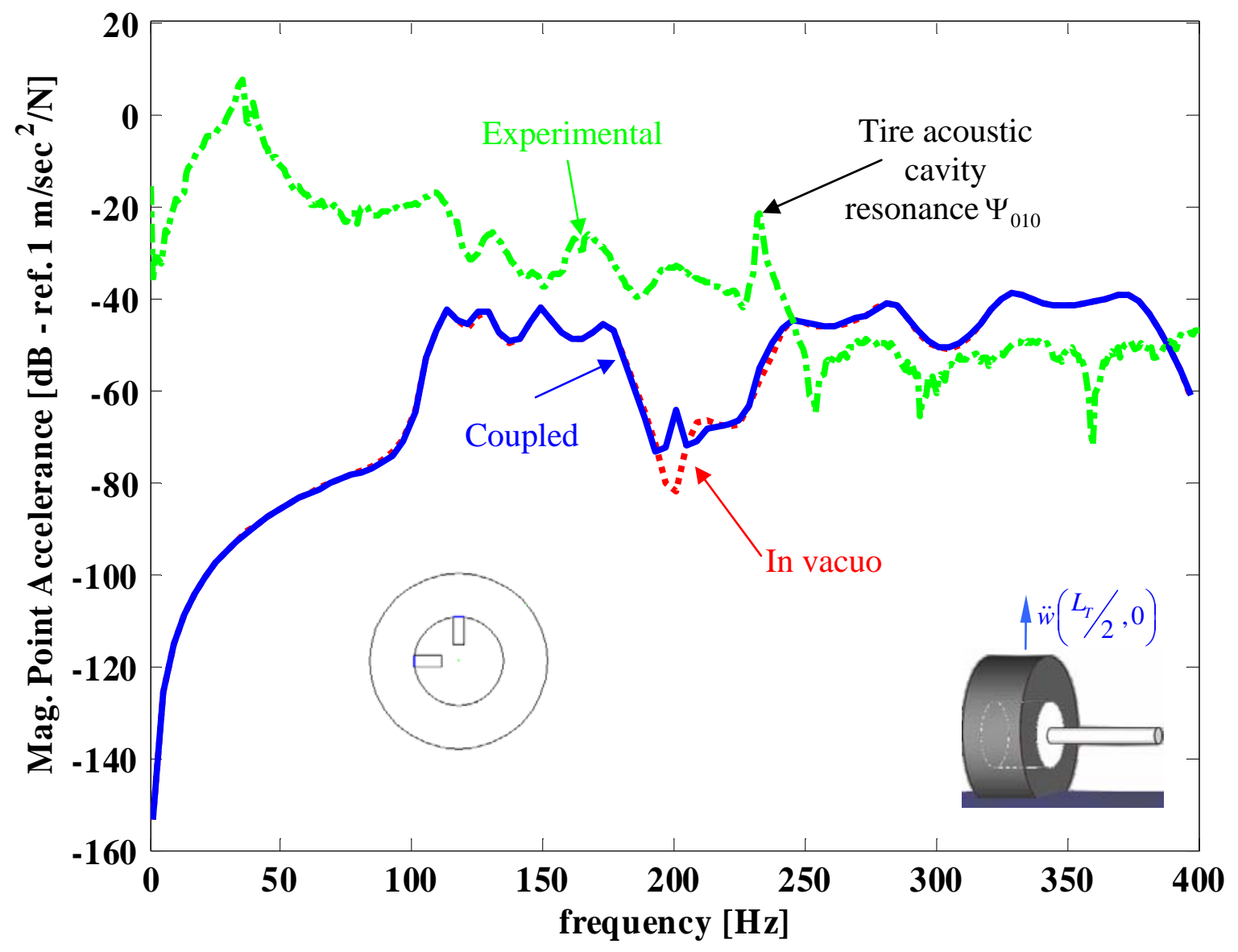

Figure 4.19: Magnitude of the point accelerance.

The result of moving the both secondary cavities in the azimuth direction an amount of $45^{\circ}$ is shown in Figures 4.20 and 4.21. The parameters of the secondary cavities are the ones selected in the previous study. The secondary cavities are shown to be working efficiently even in this adverse condition. 


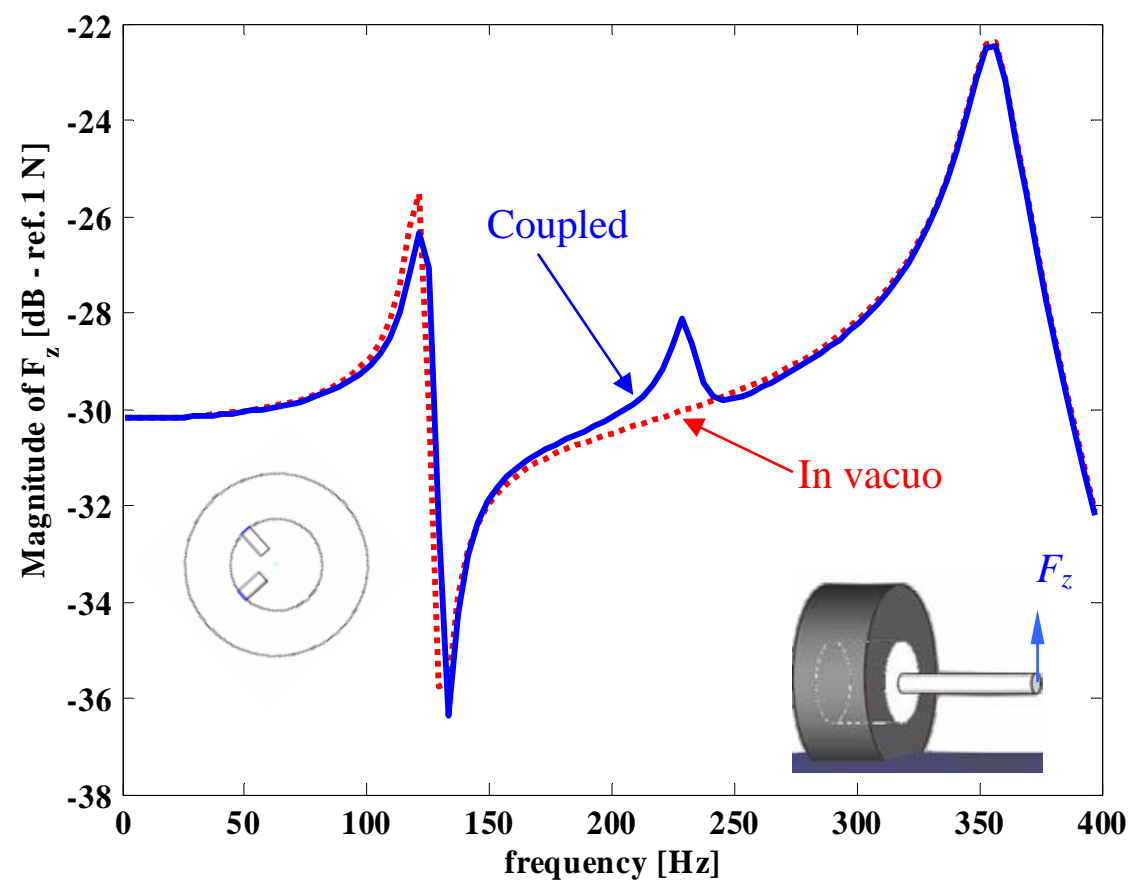

Figure 4.20: Magnitude of the vertical force at the spindle for different secondary cavity azimuth position.

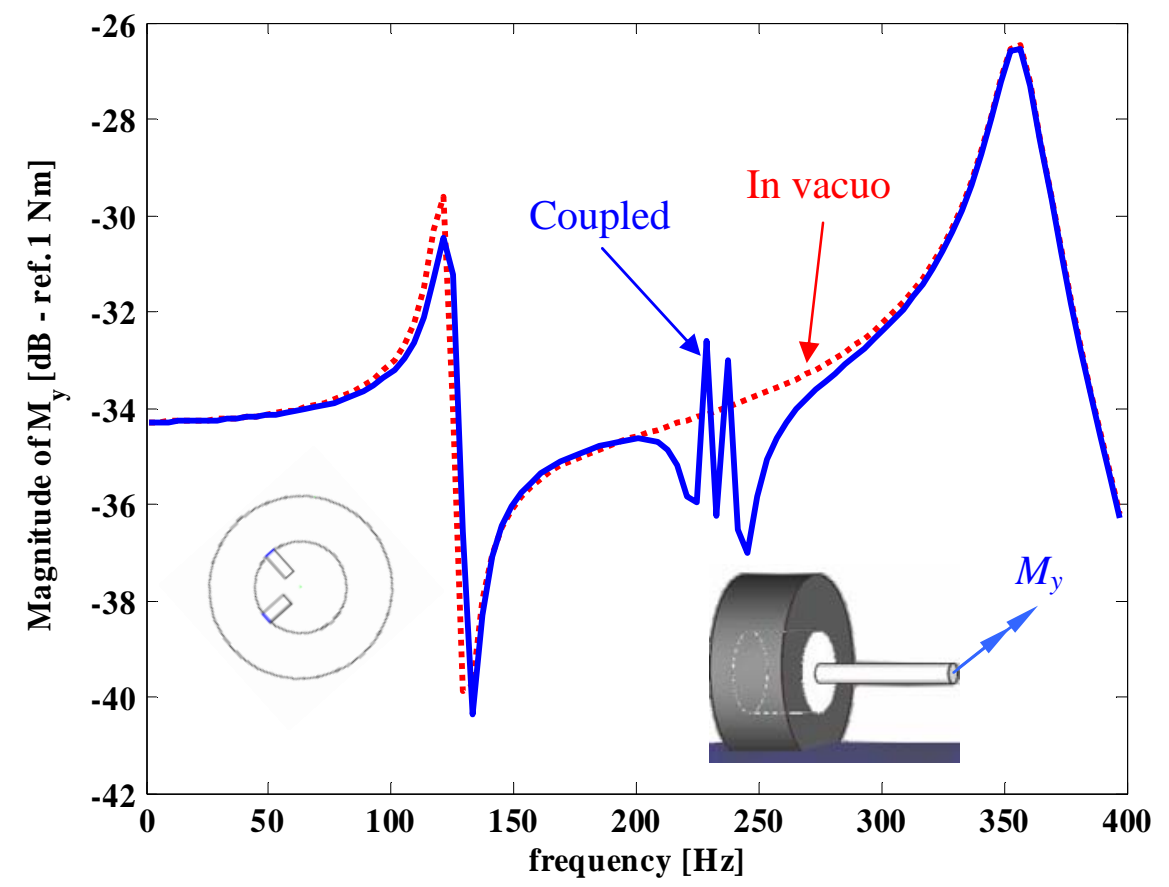

Figure 4.21: Magnitude of the horizontal moment at the spindle for different secondary cavity azimuth position. 


\subsection{DAMPING APPROACH USING VISCOELASTIC SCREENS}

The second control approach proposed in this study is based on introducing damping into the acoustic cavity resonance directly without changing its resonance frequency. This is a new experimental concept proposed in order to reduce the tire resonance. The noise levels produced for the tire acoustic cavity resonance can be attenuated by using "viscoelastic screens" placed inside the tire. Figure 4.22 shows the device, which consists of an elastic "ring" that is mounted in the inside of the wheel. Multiple screens are attached to the elastic ring in a circumferential pattern. The screen can be of different shapes such as strings, perforated membrane, and so forth. The screens and the elastic ring can be made of a viscoelastic material, capable of dissipating vibrational energy, i.e. high loss factor.

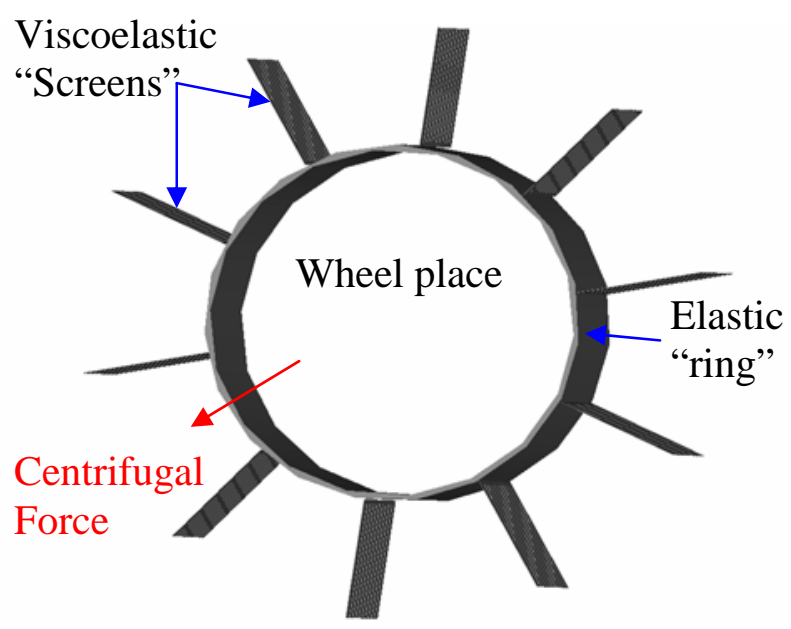

Figure 4.22: Hold and viscoelastic screens arranged in the tangential direction.

The working principle of this innovative approach is described here with the help of Figures 4.23 and 4.24. The "ring" can be easily mounted on the inside surface of the wheel. As the wheel rotates, the "ring" will rotate with the wheel, i.e. no slip between device and wheel. As the vehicle moves and the wheel rotate, a centrifugal force is 
developed in the screens. This centrifugal force unfolds the viscoelastic screens, i.e. the screens span in the radial direction. Figure 4.23 shows a side view of the device mounted in the tire-wheel assembly. Figure 4.24 shows a cut on a tire where the unfolded screen can be seen. The tire cavity acoustic resonances are the result of waves propagating in the circumferential direction (both clock and anti-clock wise). Thus, the "radial screens" are normal to the wave propagation direction and are easily excited. Therefore, the vibration of the viscoelastic screens dissipates the acoustic energy in the tire cavity.

The implementation of this device has the clear advantage that it does not affect in anyway the tire structure, i.e. the device can be applied to old and new tire systems.

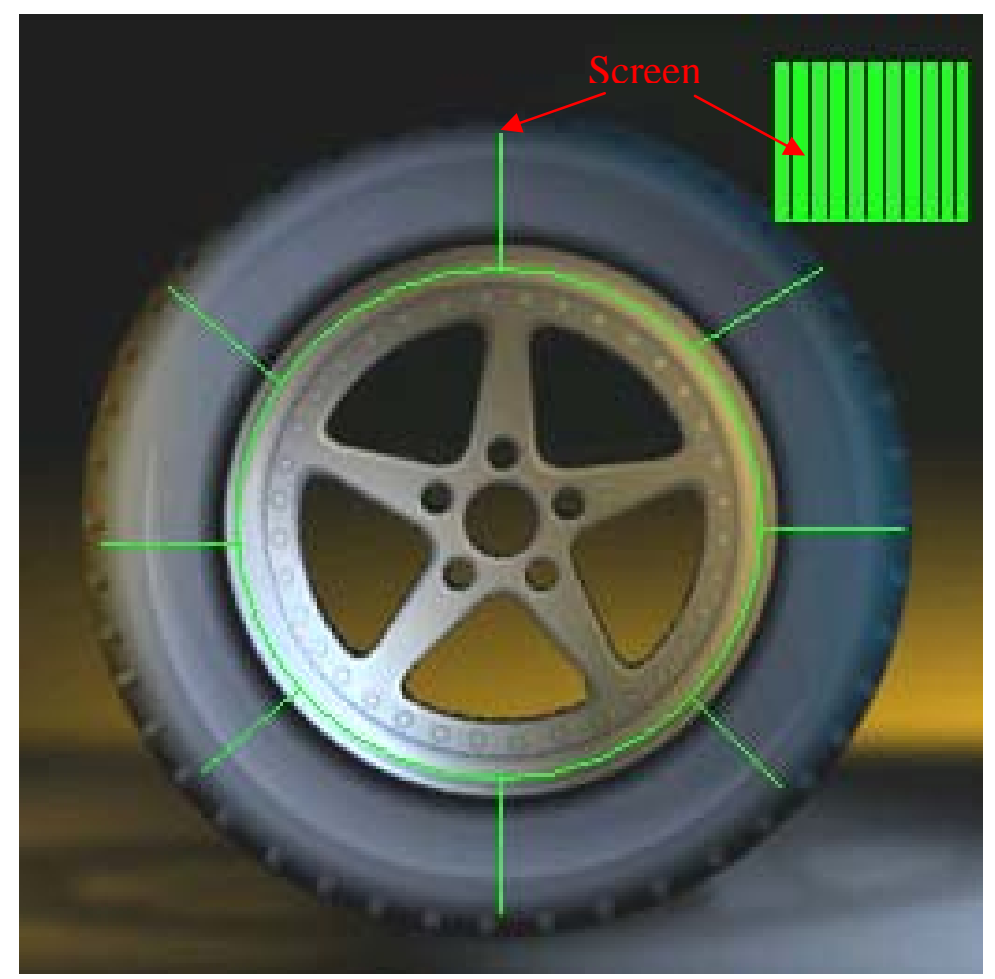

Figure 4.23: Device placed into the tire cavity. 


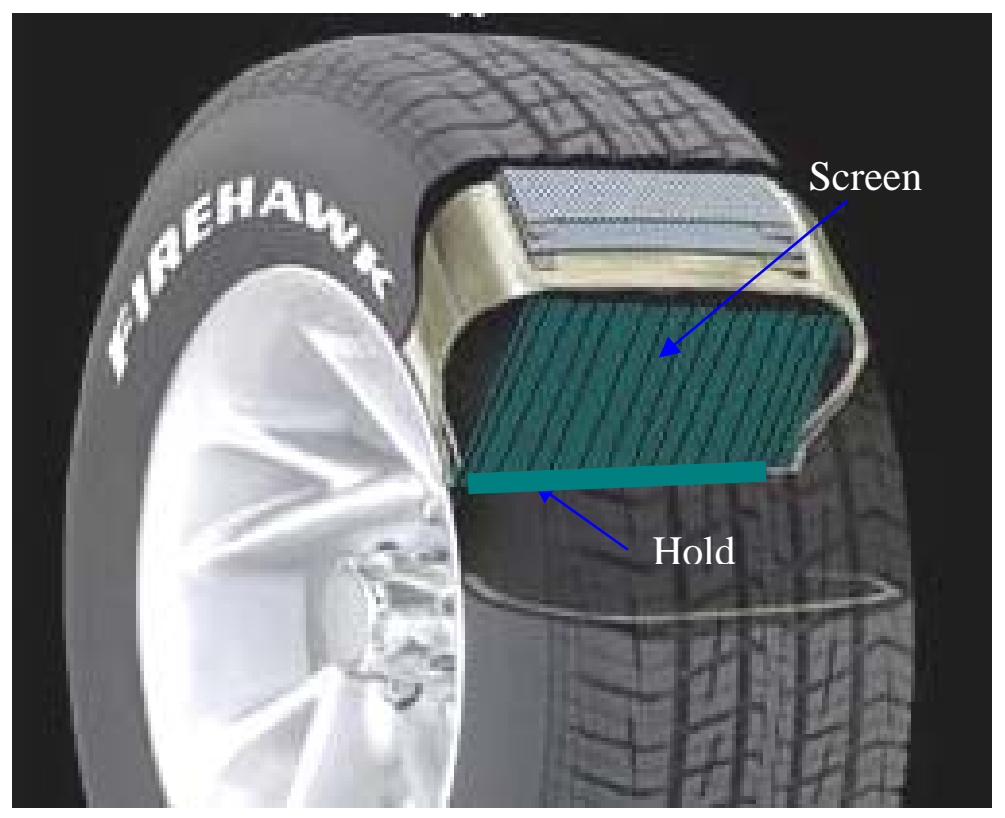

Figure 4.24: Tire with acoustic treatment.

A simple experiment was performed in order to show the potential noise level reduction of the concept in an acoustic cavity with screens. A picture of the experiment set up is shown in Figure 4.26. It consists of a long square cross section duct closed at both ends.

This setup represents or is equivalent to an unwrap tire cavity. This is an approximation of the true tire cavity problem. Eight screens were mounted along the duct. These screens were made of thin ( $1 \mathrm{~mm}$ thickness) rubber sheet as seen in Figure 4.25. The screens were cut into multiple identical slices.

The acoustic field inside of the cavity was exited by a speaker mounted at one end of the duct as seen in Figure 4.26. An array of microphones was mounted along the duct to monitor the sound field. The speaker was driven with white-noise over [0-800] $\mathrm{Hz}$ frequency band and the microphone spectra recorded. As an example, Figure 4.27 shows the spectra with and without screens. This figure shows that screens are a very effective concept to absorb the cavity acoustic energy. From these plots, the acoustic loss factor (or damping) at resonance was estimated using the half-power method, Ewins (1985) and 
Maia and Silva (1997). The reduction of the spectral peaks can be used as an estimate of potential sound level reduction at resonance. However, the increase in the loss factor can also be used to estimate potential noise level reduction.

$$
E[d B]=10 \times \log \left(\frac{\eta_{r}^{(i)}}{\bar{\eta}_{r}^{(i)}}\right)
$$

where

$\eta_{r}^{(i)}$ : Loss factor of the $r^{t h}$ acoustic mode measured on the $i^{t h}$ microphone on the different configurations.

$\bar{\eta}_{r}^{(i)}$ : Loss factor of the $r^{t h}$ acoustic mode measured on the $i^{t h}$ microphone on the reference configuration.

The results from the experiments are summarized in Table 4.1 only for the second resonance frequency. This table shows the loss factor and estimated noise level reductions for several configurations. In addition, the shift in the resonance frequency produced by the screens is indicated in parenthesis in the $2^{\text {nd }}$ row. The "Ref. column" corresponds to the case without screens, i.e. reference configuration. The "No slices" column corresponds to the cavity with screens but without cuts. The other columns present a study of the screens with increasing number of slices. The results show that (a) Increasing the number of slices on the screen decrease the attenuation of noise and (b) the noise reduction increases with the number of screens. 
Table 4.1: Acoustic damping at $2^{\text {nd }}$ resonance and expected noise reduction levels.

\begin{tabular}{|c|c|c|c|c|c|c|}
\hline & \multicolumn{6}{|c|}{ Viscoelastic Screens } \\
\hline & $\begin{array}{c}\text { No } \\
\text { Screen } \\
\text { Ref. }\end{array}$ & $\begin{array}{c}\mathbf{8} \\
\text { Screns } \\
\text { No Slices }\end{array}$ & $\begin{array}{c}\mathbf{8} \\
\text { Screens } \\
\mathbf{6} \text { Slices }\end{array}$ & $\begin{array}{c}\mathbf{8} \\
\text { Screens } \\
\mathbf{1 2} \text { Slices }\end{array}$ & $\begin{array}{c}\mathbf{8} \\
\text { Screens } \\
\mathbf{2 4} \text { Slices }\end{array}$ & $\begin{array}{c}\mathbf{4} \\
\text { 5creens } \\
\mathbf{2 4} \text { Slices }\end{array}$ \\
\hline$\eta_{2}$ & 0.0367 & 0.412 & 0.192 & 0.106 & 0.073 & 0.05 \\
$($ Shift $)$ & $(-98 \mathrm{~Hz})$ & $(-44 \mathrm{~Hz})$ & $(-21 \mathrm{~Hz})$ & $(-9 \mathrm{~Hz})$ & $(-4 \mathrm{~Hz})$ \\
\hline $\begin{array}{c}\text { Expected } \\
\text { reduction } \\
\text { [dB] }\end{array}$ & - & 21.0 & 14.4 & 9.2 & 3.5 & 2.7 \\
\hline
\end{tabular}

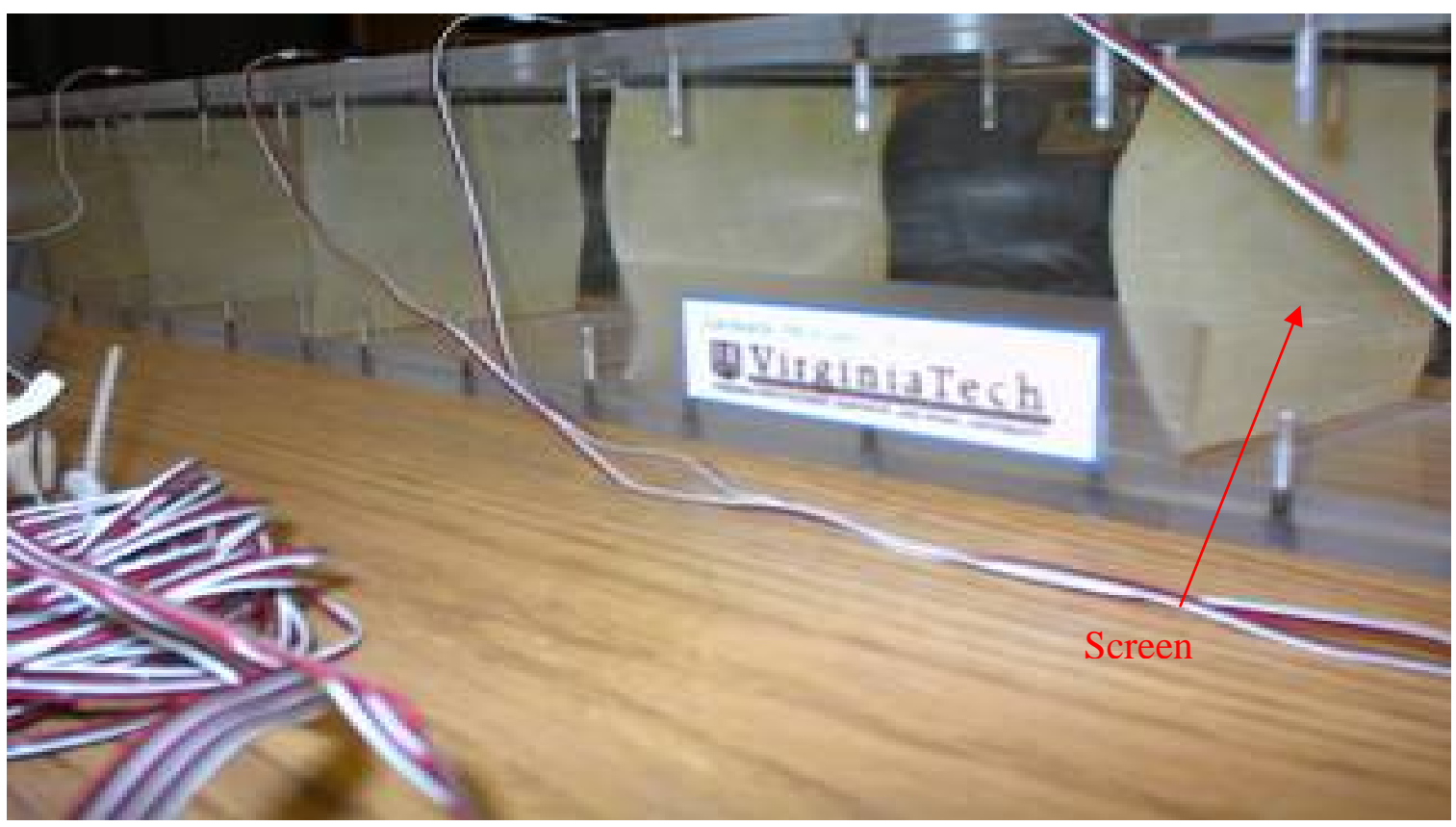

Figure 4.25: View of the viscoelastic screens inside of the main cavity. 


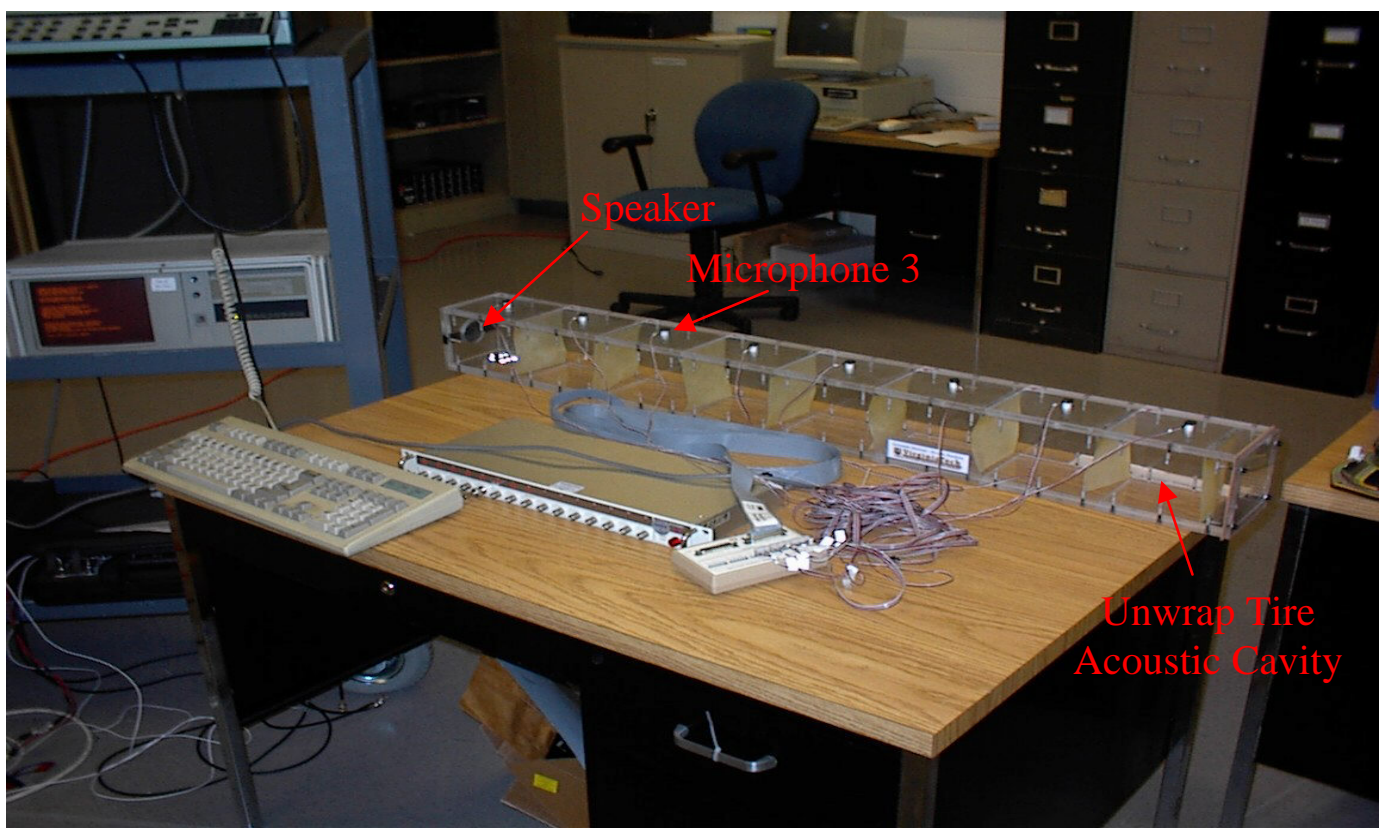

Figure 4.26: Experiment to validate the concept of the viscoelastic screens.

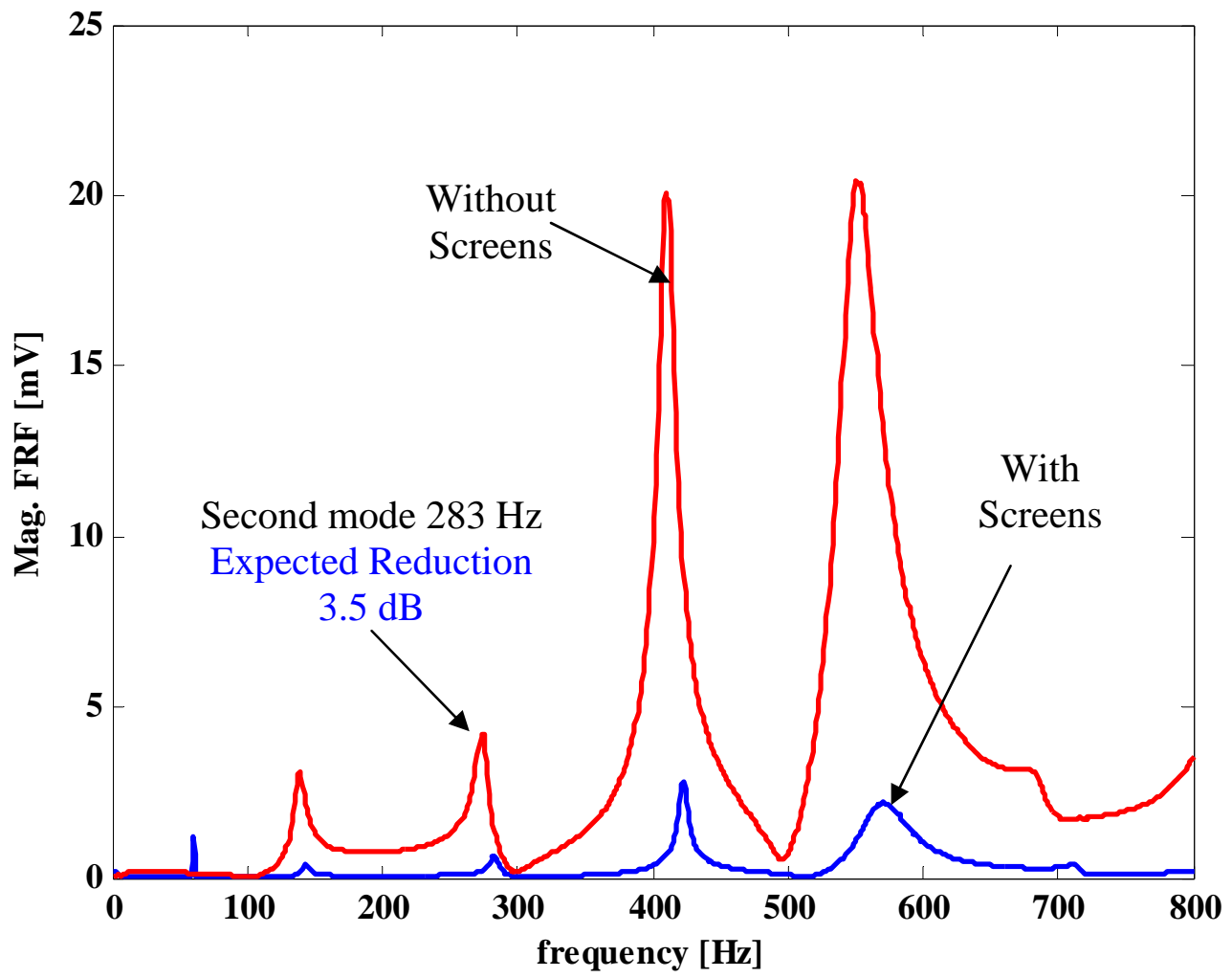

Figure 4.27: FRF Magnitudes with 8 Screens - 24 Slices (blue line) and without screens (red line) at Microphone 3. 


\subsection{VISCOELASTIC SCREENS CONTROL APPROACH}

The idea of the use of the viscoelastic screens concept is modeled easily by adding viscous damping into the tire acoustic cavity. The acoustic damping was introduced into the model by using the "complex speed" of sound, which is defined as

$$
\hat{c}=c\left(1+i \varsigma_{A}\right)
$$

where,

$\varsigma_{A}:$ is the acoustic loss factor

$i$ : is the imaginary unit.

The effect of the viscoelastic screens in the free and deformed model were investigated for an acoustic loss factor of $1 \%$, and $3 \%$ in the [0-400] Hz frequency range.

Figures 4.28 and 4.29 show the force and moment at the spindle for the free tire model, respectively. The addition of acoustic damping helps to damp out the energy of the acoustic cavity resonance i.e. $\zeta_{A}: 3 \%$. Therefore, the viscoelastic screens are an effective method to control the tire cavity resonance effect in the forces at the spindle. 


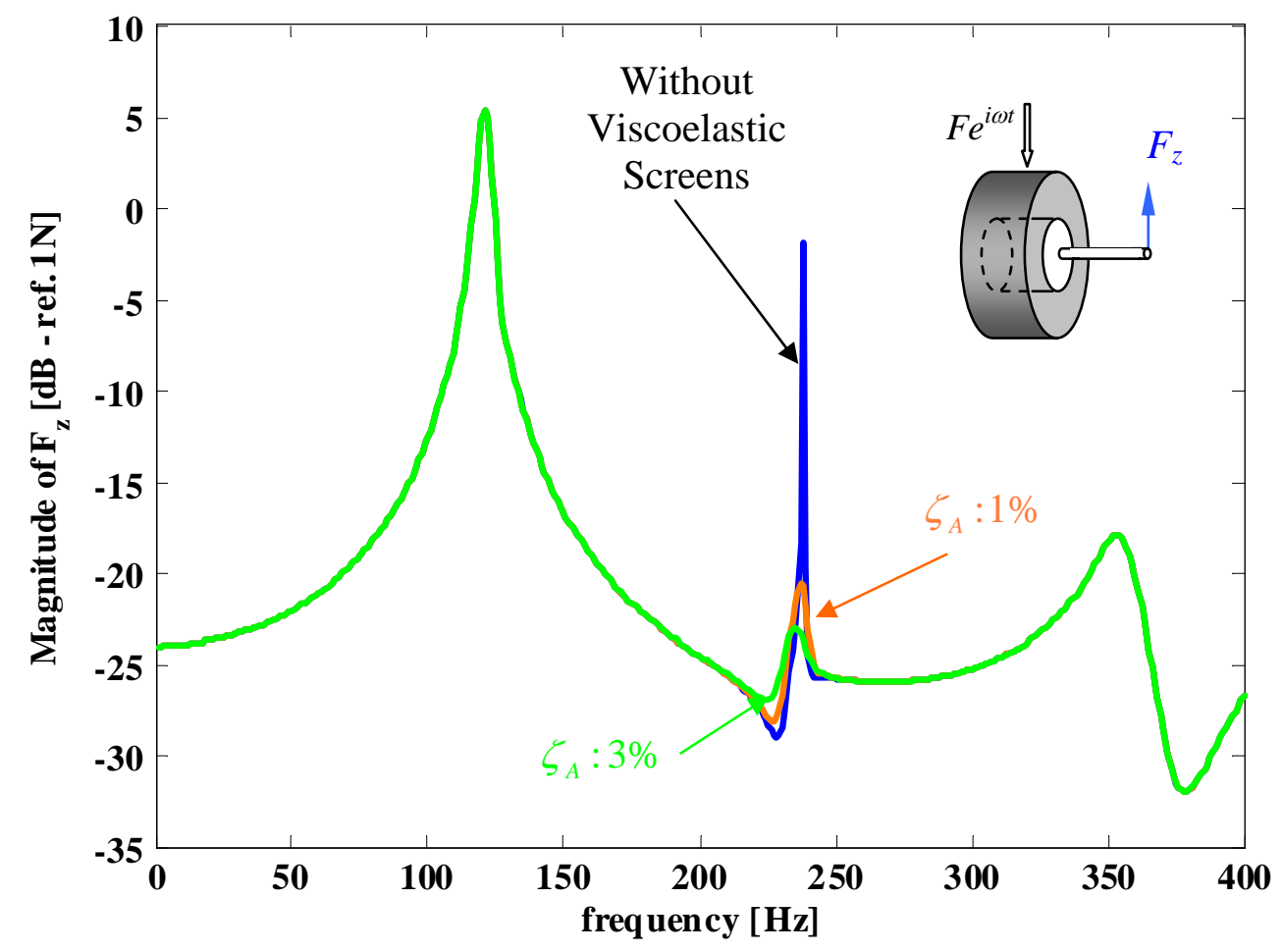

Figure 4.28: Magnitude of the vertical force at the spindle.

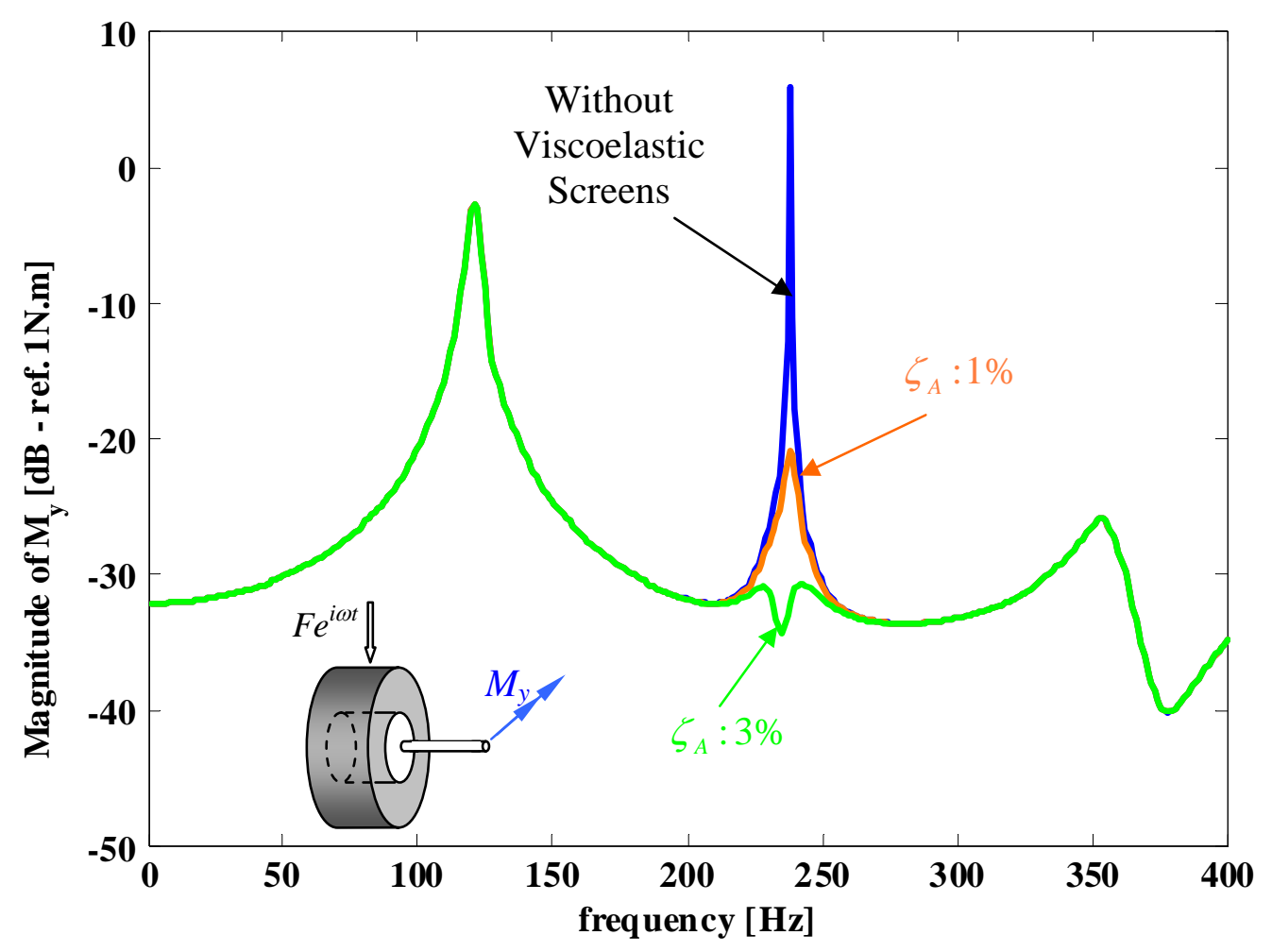

Figure 4.29: Magnitude of the horizontal moment at the spindle. 
Figure 4.30 and 4.31 show the forces at the vehicle spindle for the deformed tire model. Also, in this case an important force reduction at the cavity resonance is observed.

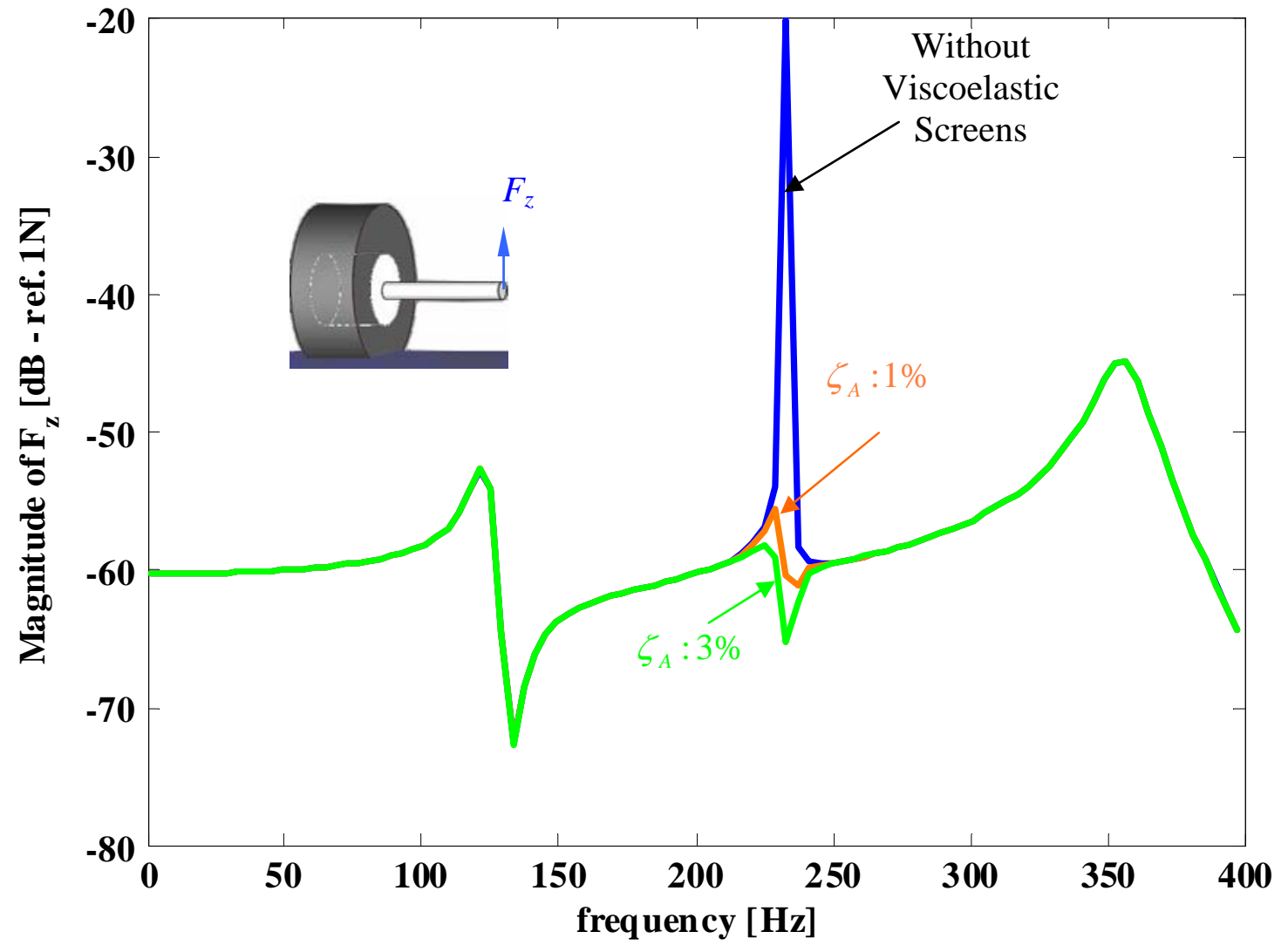

Figure 4.30: Magnitude of the vertical force at the spindle. 


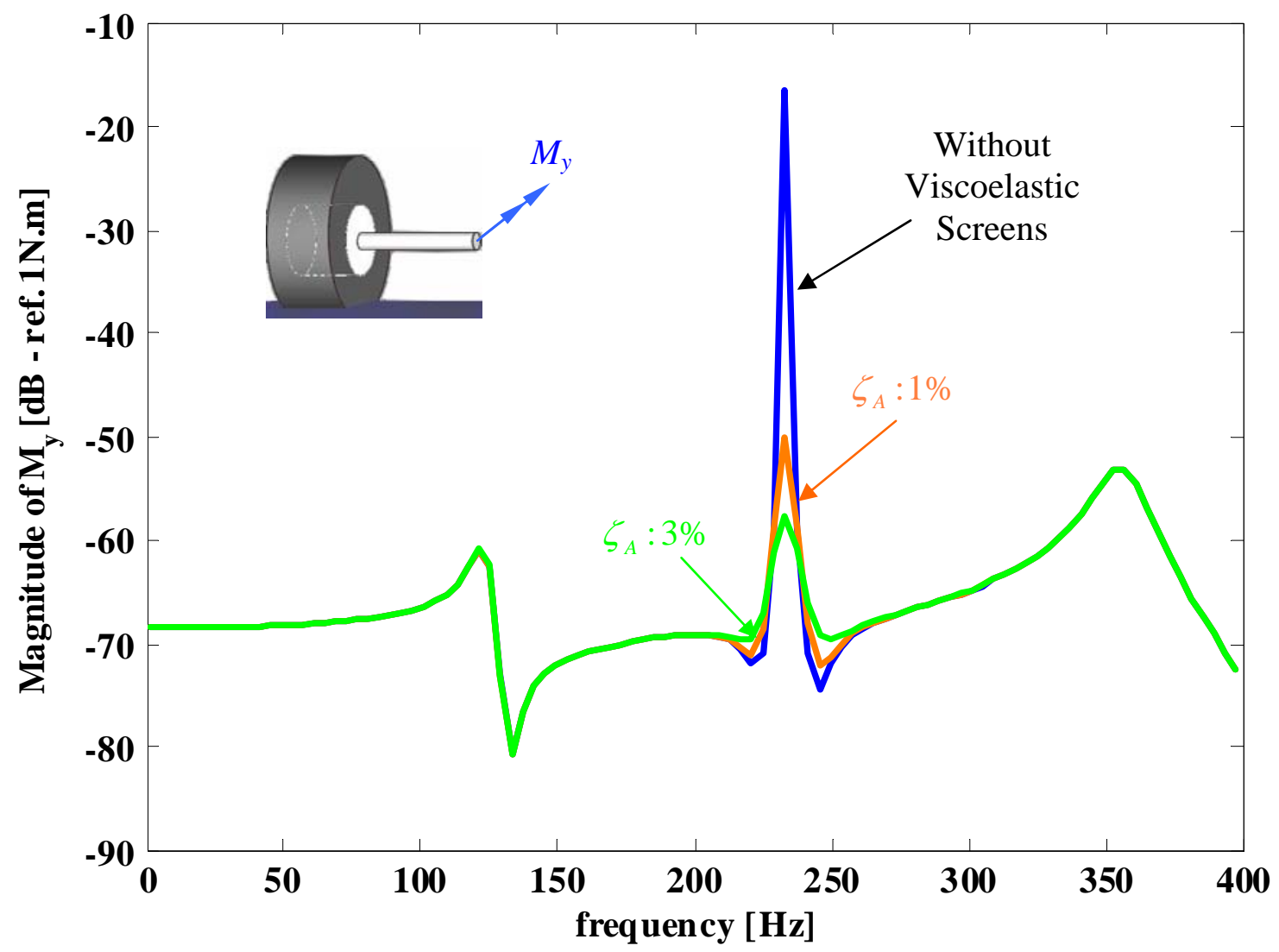

Figure 4.31: Magnitude of the horizontal moment at the spindle.

The viscoelastic screen approach is a simple and practical approach. The results in both the free and deformed tire model show the capability of the viscoelastic screens to control the dominant tire acoustic cavity resonance. In the free tire model the level of reduction achieved permit to eliminate the effect of the cavity resonance. In the deformed tire case where the cavity resonance showed to have a larger effect relative to the structural resonances than in the free tire case, the cavity effect is controlled to have a magnitude smaller than the structural resonances. The techniques presented in this chapter show to be very effective to control the tire acoustic cavity resonance. 


\section{CHAPTER 5: SYNOPSIS, CONCLUSIONS, AND RECOMMENDATIONS FOR FUTURE WORK}

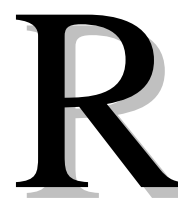

eview of the open literature revealed very limited work in the problem of interior vehicle noise due to the tire acoustic cavity resonance. This cavity resonance problem has only recently been discovered and it is not yet well understood. The open literature offers simple models to predict only the natural frequencies of the tire cavity and qualitatively predict the direction of the forces at the vehicle spindle. Most of the modeling efforts to compute the tire dynamics have focused on FE techniques that hamper the understanding of the physics of the tire-cavity coupling problem. There is also a lack of well understood and validated control approaches. Therefore, this dissertation presents analytical closed form solutions to help gaining insight into the coupling phenomenon and subsequently permits the investigation and development of practical techniques to control the problem.

The modeling of the tire structure includes the formulation for the "un-deformed or free" and "deformed" tire cases. A critical component of the model is the formulation to compute the forces at the spindle from the structural modal responses. The "free tire" is the model of the unsupported tire. In this model, the external excitation is a harmonic point force acting normal to the external surface of the tire. The "deformed tire" development includes the modeling of the contact patch deformation. Afterward, a harmonic vertical motion of the tire is superimposed that results in an effective load acting on the tire and the associated dynamic response. Lastly, numerical results for the tire models are shown. Validation of both "free" and "deformed" structural tire models is also included by comparison with experimental results obtained from the experimental work done by Yamauchi and Akiyoshi (2002). 
To account for the tire acoustic cavity in the structural response, an expression for the acoustic pressure produced by the motion of the tire is derived in this effort. To this end, the acoustic mode shapes and natural frequencies of the tire cavity are computed. The eigenproperties are then used to solve for the Green's functions, which allows to compute the acoustic pressure due to the tire motion. The modeling of the tire acoustic cavity is included in the structural models developed previously. The "free tire" and "deformed tire” models are evaluated numerically and validated by comparison to experimental results from the open literature.

It is shown in this work that the tire acoustic cavity dynamics effect results in a sharp increase of the spindle forces around the tyre cavity resonance. These forces transmitted to the rest of the car structure contribute adversely to the vehicle interior noise. Therefore, the tyre cavity resonance effect must be reduced in order to control the tire contribution to the structural born noise. The analysis and modeling of two feasible approaches to control the tyre acoustic cavity resonances are proposed and investigated in this effort. The approaches are analytically modeled and included into both the "free" and “deformed” tire formulations.

The first approach is the incorporation of secondary acoustic cavities to detune and damp out the main tire cavity resonance energy. The second approach is the addition of damping directly into the tire cavity. It is achieved by placing viscoelastic screens into the tire acoustic cavity. The damping is modeled by including the complex speed of sound in the tire models. The use of viscoelastic screens is a novel technique to control the tire acoustic resonances. 


\subsection{CONCLUSIONS}

The most significant contributions obtained during this research effort are presented in this section. The analysis of the results permits to establish the following foremost conclusions:

Closed form solution models of the acoustic cavity problem were developed. The models capture the physics of the problem as validated by comparisons against experimental results. The models allowed to gain insight into the physics of the problem as attested by the follow up conclusions.

Though the tire model is a modally rich structure, the so called shell "bending modes”, i.e. $n=1$, are the only ones capable to transmit forces to the spindle. In fact, there are only two modes, i.e. radial $(1,1)$ and tangential $(1,1)$, that results in spindle forces in the low frequency range of $[0-400] \mathrm{Hz}$

$>$ In the free tire model driven by a point force, the forces at the spindle are dominated by a single mode. i.e. radial $(1,1)$. On the other hand, the deformed tire model shows that both the radial $(1,1)$ and tangential $(1,1)$ modes contributed nearly the same to the spindle forces.

The acoustic cavity resonance was shown to have a very dominant effect in the response of the tire models. The spindle forces obtained from simulation of the "free tire" model shows that the cavity resonance is as important as the structural tire resonances. For the "deformed tire”, the tire acoustic cavity resonance is the most dominant component of the spindle forces and significantly higher than the structural resonance contributions.

The model also revealed that the high structural damping of tire rubber compound does not have any effect in the tire acoustic cavity resonance. Therefore, the structural damping does not help to damp out energy at the tire acoustic resonance. 
The techniques proposed in this dissertation to suppress the adverse effects of the acoustic cavity in the tire response, i.e. forces at the spindle, show to be very effective and can be easily implemented in practice. The secondary cavity approach requires little damping to attenuate the spindle forces due to the tire acoustic resonance. The viscoelastic screen concept was also shown theoretically and throughout a simple experiment to be a very effective method to absorb the cavity acoustic energy.

\subsection{RECOMMENDATIONS FOR FUTURE WORK}

Though the models developed here are very useful tools to predict the response, understand the dynamics, and investigate control options for the acoustic cavity problem, there are additional research issues or endeavors worthy of further studies. They are:

The experimental implementation of tire acoustic resonance control techniques is an immediate research issue. The control concepts could be first tested in a controlled laboratory setup and later evaluated in an actual vehicle road test.

The model is amenable to incorporate more control techniques. A control option that could be interesting to investigate is the use of the Bragg phenomenon. This phenomenon is based in destructive interference that can be achieved by a cavity with wavy walls. For the case of a tyre, this technique could be realized by using evenly distributed flaps mounted in the vehicle wheel.

The rigid component of the tire model could be modified to account for flexibilities, i.e. tire side plates and spindle. Therefore, the model of the forces will account for the new dynamic of these flexible parts.

The tire model does not predict the split of resonance frequency when the tire is deformed. In fact, in this dissertation only the acoustic mode excited by the vertical motion of the tire is included in the model. The accuracy of the model can be 
Leonardo Molisani Chapter 5: Synopsis, Conclusions, and Recommendations for Future Work

improved by incorporating the effect of the frequency split of the tire cavity resonance. 


\section{REFERENCES}

Belluzzo, D., Mancosu, F., Sangalli, R., Cheli, F., and Bruni, S., (2002) New Predictive Model for the Study of Vertical Forces (up to $250 \mathrm{~Hz}$ ) Induced on tha Tire Hub by Road Irregularities, Tire Science and Technology, TSTCA, Vol. $30 \mathrm{~N}^{0}$ 1, January - March 2002, pp. 2-18.

Beranek, L., and Vér, I., (1992) Noise and Vibration Control Engineering, John Wiley \& Sons, Inc.

Blevins, R., (1995) Formulas for Natural Frequency and Mode Shape, Krieger Publishing Company.

Chai, J., Kim, Y. and Kang K., (2002) Vehicle Tire Noise/Vibration Characteristics Analysis Using SEA (Statistical Energy Analysis), The 2002 International Congress and Exposition on Noise Control Engineering, Dearborn, MI, USA.

Clayton, W. and Sait-Cyr R.,(1998) Incorporation of the Tyre Air Cavity Resonance into the Modal Tyre Model, Conference.

Darnell, I, and Kestler, A., (2002) Analysis of Tire Models for Rolling on a deformable Substrate, Tire Science and Technology, TSTCA, Vol. 30 N 3, July - September 2002, pp. 180-197.

Ewins, J. D., (1985) Modal Testing: Theory and Practice, Research Studies Press Ltd, BRÜEL \& KÆR.

Fahy, F., (2000) Sound and Structural Vibration, Academic Press.

Greenspon, J. E., (1958) Flexural Vibrations of a Thick-Walled Circular Cylinder, Proc. $3^{\text {rd }}$ U.S. Nat. Congr. of Appl. Mech., Providence, R.I., pp163-173. 
Griffel, D. H., (1985) Applied Functional Analysis, Dover Publications, Inc.

Hallez, R. Smith, J., and Burdisso, R, (2000) Control of Higher Order Modes in Ducts using Arrays of Herschel-Quincke Waveguides, ASME 2000, Nov. 5-10, Orlando.

Hansen, T., (1999) Plane-Wave Theory of Time-Domain Fields, IEEE Press.

Ingard U. and Ising H., (1967) Acoustic Non linearity of an Orifice, Journal of Acoustical Society of America, 42(1), pp. 6-17.

Junger, M. C. and Feit, D., (1986) Sound, Structures and Their Interaction, $2^{\text {nd }}$ Edition, MIT Press, Boston.

Kinsler, L et all, (2000) Fundamentals of Acoustics, John Wiley \& Son, Inc.

Klein, P., (2002) Tyre Noise Horn Effect on an Absorbing Road Surface - 2D Analytical Modelling Using the Multipole Synthesis, The 2002 International Congress and Exposition on Noise Control Engineering, Dearborn, MI, USA.

Kreyszig, E., (1978) Introductory Functional Analysis with Aplications, John Wiley \& Son, Inc.

Kuijpers, A., (2001) Tyre/Road Noise Models in the last Two Decades: a critical evaluation, InterNoise 2001 Conference

Lawrenson, C. et al., (1998) The Solution For The Propagation Of Sound In A Toroidal Waveguide With Driven Walls, J. Acoustic Soc. Am., 103(3), pp. 1253-1260.

Leissa, A., (1993) Vibration of Shells, Acoustical Society of America. 
Maia, N. M. M. and Silva, M. M. J., (1997) Theoretical and Experimental Modal Analysis, Research Studies Press.

McLachlan, N., (1961) Bessel Functions for Engineers, Oxford University Press.

Meirovitch, L., (2001) Fundamentals of Vibrations, McGraw-Hill.

Molisani, L. and Burdisso, R., (2000) Modeling of Tire Acoustic Cavity and the Effect on Vehicle Interior Noise, Virginia Tech Final Report, Proposal \# 00-0990-12.

Nackenhorst, U., (2001) Numerical Analysis of the Tyre Rolling Noise Radiation-A State of the Art Review, InterNoise 2001 Conference.

Nakajima, Y. et all, (1992) Application of the Boundary Element Method and Modal Analysis to Tyre Acoustic Problems, Tyre Science and Technology, TSTCA, Vol. 21, $\mathrm{N}^{\mathrm{O}}$ 2 April-June, pp. 66-90.

Naylor, W. and Sell, George, (2000) Linear Operator Theory in Engineering and Science, Applied Mathematical Sciences, Springer.

Olatunbosum, O. and Burke A., (2002) Finite Element Modelling of Rotating Tires in the Time Domain, Tire Science and Technology, TSTCA, Vol. $30 \mathrm{~N}^{\mathrm{o}}$ 1, January - March 2002, pp. 19-33.

Pietrzyk, A., (2001) Prediction Of The Dynamic Response Of A Tyre, InterNoise 2001 Conference.

Rao, S. S., (1990) Mechanical Vibrations, Addison-Wesley Publishing Company.

Roach, G. F., (1970) Green’s Functions, Van Nostrand Reinhold Company. 
Rochat, L., (2002) Tyre/Road Noise, The 2002 International Congress and Exposition on Noise Control Engineering, Dearborn, MI, USA

Sakata, T et. all., (1990) Effect of Tyre Cavity Resonance on Vehicle Road Noise, Tyre Science and Technology, Vol .18. pp 68-79.

Sandberg, U., (2001) Noise Emissions of road Vehicles - Effects of Regulations, NOISE/NEWS INTERNATIONAL Magazine Volume 9, Number 3 - September of 2001.

Sandberg, U., (2002) Review of Current Standarization Efforts with Regard toTyre/Road Noise, The 2002 International Congress and Exposition on Noise Control Engineering, Dearborn, MI, USA

Sandberg, U. and Ejsmont J., (2002) Tyre/Road Noise reference Book, First Edition, Informex, Modena, Poland.

Sobhanie, M., (2003) Road Load Analysis, Tire Science and Technology, TSTCA, Vol. $31 \mathrm{~N}^{\mathrm{o}}$ 1, January - March 2003, pp. 19-38.

Soedel, W., (1993) Vibration of Shells and Plates, Marcel Dekker, Inc.

Thompson, J., (1995) Plane Wave Resonance in the air Cavity as a Vehicle Interior Noise Source Tyre Science and Technology, Vol .23. pp 2-10.

Timoshenko, S. and Woinowsky-Krieger, S., (1959) Theory of Plates and Shells, McGraw-Hill.

Tsihlas, D., (2000) Personal communication for the Proposal\# 00-0990-12, Spring 2000.

Ventsel, E. and Krauthammer, T., (2001) Thin Plates and Shells, Marcel Dekker, Inc. 
Watson, G., (1958) A Treatise on the Theory of Bessel Functions, Cambridge at the University Press.

Watson, G., (1958) A Treatise on the Theory of Bessel Functions, Cambridge at the University Press.

Wu, T. W., (2000) Boundary Element Acoustics, Advances in Boundary Elements Series, WITPress.

Yamauchi H. and Akiyoshi Y., (2002) Theoretical Analysis of tire Acoustic Cavity Noise and Proposal of Improvement Techniques, Society of Automotive Engineers of Japan, Inc. and Elsevier Science.

Zauderer, E.,(1989) Partial Differential Equations of Applied Mathematics, Second Edition, John Wiley \& Sons, Inc. 


\section{APPENDIX A: TIRE SiZE NOMENCLATURE}

In this appendix an explanation of the tire dimensions nomenclature is presented. The schematics for a tire dimensions is shown in Figure A.1. The tire width in millimeters is represented by $\boldsymbol{B}$ in the figure. The tire section height is called $\boldsymbol{H}$, the Rim diameter is $\boldsymbol{d}$, and the tire outer diameter is $\boldsymbol{D}$.

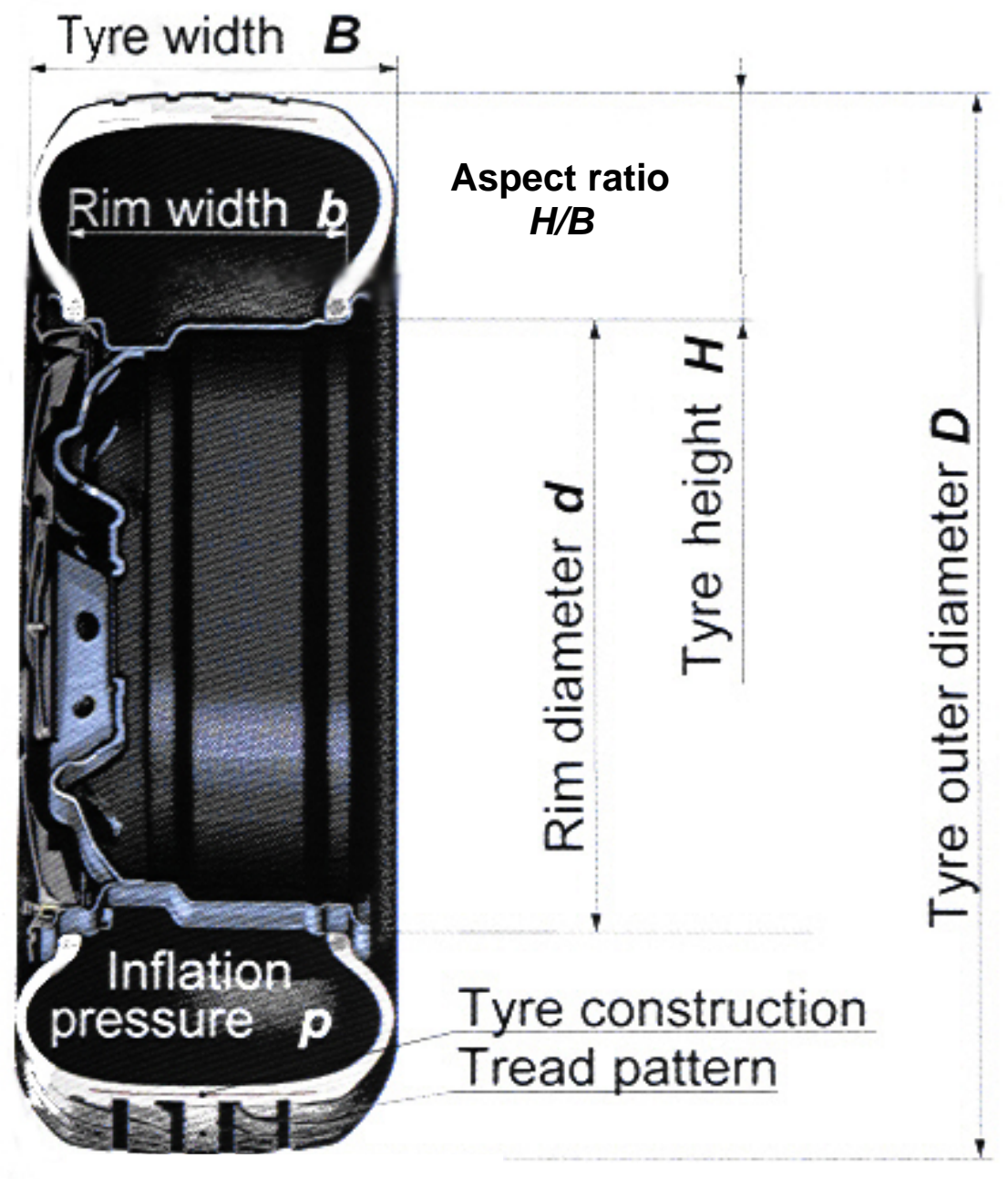

Figure A.1: Schematic tire dimension. 
In most of the cases, the tire size is given by 3 numbers and a letter. The definitions in the tire nomenclature are shown in Figure A.2.

\section{5 / 65 R 15}

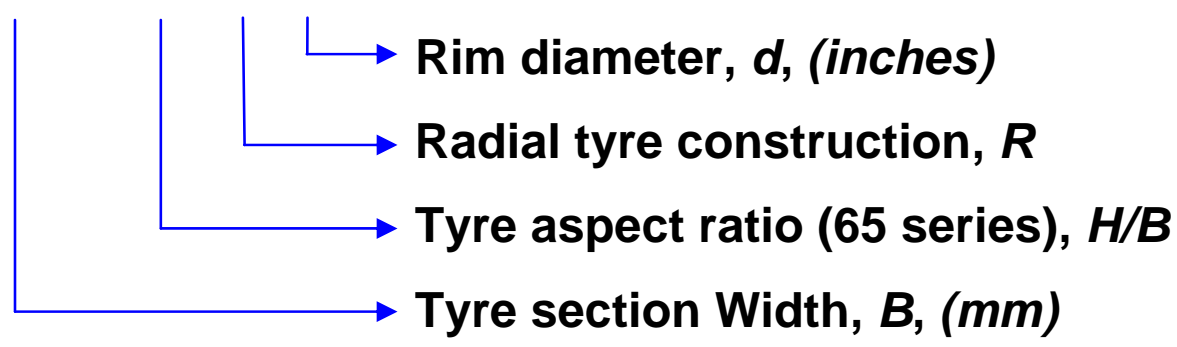

Figure A.2: Tire nomenclature definitions.

The tire 195/65 R15 is used in the simulation on this work to validate the model with experimental data. From the tire size specification the following parameters are solved to input the code.

The tire with $L_{T}$

$$
L_{T}=B=195 \mathrm{~mm}=0.195 \mathrm{~m}
$$

The tire inner radius

$$
b=\frac{d}{2}=\frac{15 i n}{2}=7.5 i n=0.191 m
$$

The tire outer radius

$$
a=b+H=b+0.65 B=0.191 m+0.127 m=0.318 m
$$


The material properties of the tire for the numerical simulations were provided by Dimitri Tsihlas from Michelin North America (Tsihlas, 2001). They are:

Table A.1: Tire’s material and geometrical parameters

\begin{tabular}{|c|c|}
\hline Parameter & Description \\
\hline$L_{T}=0.195 m(7.68 \mathrm{in})$ & Tire Width \\
\hline $\boldsymbol{a}=0.318 \mathrm{~m}(12.52 \mathrm{in})$ & Outer Radius \\
\hline $\boldsymbol{b}=0.191 \mathrm{~m}(7.52 \mathrm{in})$ & Inner Radius \\
\hline $\boldsymbol{h}_{\text {Shell }}=0.015 \mathrm{~m}(0.59 \mathrm{in})$ & Thickness \\
\hline$d=0.05 m(1.97 \mathrm{in})$ & Spindle length \\
\hline $\boldsymbol{E}=75 \times 10^{6} \mathrm{~N} / \mathrm{m}^{2}$ & Young's Modulus \\
\hline $\boldsymbol{\rho}=1350 \mathrm{~kg} / \mathrm{m}^{3}$ & Material Density \\
\hline$\eta=0.03$ & Loss Factor \\
\hline$v=0.4$ & Poisson ratio \\
\hline
\end{tabular}




\section{APPENDIX B: SOLVING THE CONTACT PATCH EQUATIONS}

This appendix shows the calculation of the integral of equation (2.30) in Chapter 2. Equation (2.37) is repeated here in order to continue with the computation of the contact patch

$$
\begin{aligned}
& \int_{O}^{L_{T}} \int_{-\theta_{\mathrm{p}}}^{\theta_{\mathrm{p}}} d(x, \theta) \sin \left(s \frac{\pi}{L_{T}} x\right) \cos (r \theta) a d \theta d x= \\
& =\int_{O}^{L_{T}} \int_{-\theta_{\mathrm{p}}}^{\theta_{\mathrm{p}}}\left[\sum_{m}^{M} \sum_{n}^{N} W_{m n}^{(3)} \sin \left(\frac{n \pi}{L_{T}} x\right) \cos (n \theta) \sin \left(\frac{s \pi}{L_{T}} x\right) \cos (r \theta)\right] a d \theta d x
\end{aligned}
$$

Replacing in equation (B.1) the value of $d(x, \theta)$ and integrating in the axial direction, last equation becomes

$$
\begin{aligned}
& \frac{L_{T}}{s \pi}\left[1-(-1)^{s}\right]\left\{[h(t)-a] \int_{-\theta_{\mathrm{p}}}^{\theta_{\mathrm{p}}} \sec (\theta) \cos (r \theta) d \theta-a \int_{-\theta_{\mathrm{p}}}^{\theta_{\mathrm{p}}} \cos (r \theta) d \theta\right\}= \\
& =\frac{L_{T}}{2} \sum_{n=0}^{N} \int_{-\theta_{\mathrm{p}}}^{\theta_{\mathrm{p}}} W_{m n}^{(3)} \cos (n \theta) \cos (r \theta) d \theta
\end{aligned}
$$

The integrals are solved in close form. Therefore,

$$
I_{r}^{(1)}=\int_{-\theta_{\mathrm{p}}}^{\theta_{\mathrm{p}}} \sec (\theta) \cdot \cos (r \theta) d \theta=\int_{-\theta_{\mathrm{p}}}^{\theta_{\mathrm{p}}} \frac{\cos (r \theta)}{\cos (\theta)} d \theta
$$

which is 


$$
I_{r}^{(1)}=\left\{\begin{array}{ll}
2 \sum_{k=1}^{r}\left[(-1)^{r-k} \frac{\sin \left(2 k \theta_{\mathrm{p}}\right)}{2 k}\right]+(-1)^{r} \theta_{2}-2 \sum_{k=1}^{r}\left[(-1)^{r-k} \frac{\sin \left(-2 k \theta_{\mathrm{p}}\right)}{2 k}\right]+(-1)^{r} \theta_{\mathrm{p} 1}, & r: \text { odd } \\
2 \sum_{k=1}^{r}\left\{(-1)^{r-k} \sin \left[\frac{\left(2 k \theta_{\mathrm{p}}\right)}{2 k}\right] \theta_{\mathrm{p}}\right\}+(-1)^{r} \ln \left[\operatorname{tg}\left(\frac{\pi}{4}+\frac{\theta_{\mathrm{p}}}{2}\right)\right] & \\
-2 \sum_{k=1}^{r}\left\{(-1)^{r-k} \sin \left[\frac{-(2 k-1) \theta_{\mathrm{p}}}{2 k-1}\right]\right\}-(-1)^{r} \ln \left[\operatorname{tg}\left(\frac{\pi}{4}-\frac{\theta_{\mathrm{p}}}{2}\right)\right] & \\
\ln \left[\operatorname{tg}\left(\frac{\pi}{4}+\frac{\theta_{\mathrm{p}}}{2}\right)\right]-\ln \left[\operatorname{tg}\left(\frac{\pi}{4}-\frac{\theta_{\mathrm{p} 1}}{2}\right)\right], & r=0
\end{array}\right\}
$$

Then,

$$
I_{r}^{(2)}=\int_{-\theta_{\mathrm{p}}}^{\theta_{\mathrm{p}}} \cos (r \theta) d \theta=I_{r}^{(2)}=\left\{\begin{array}{c}
2 \theta_{\mathrm{p}}, r=0 \\
\frac{2 \sin \left(r \theta_{\mathrm{p}}\right)}{r}, r \neq 0
\end{array}\right\}
$$

and finally

$$
\begin{gathered}
I_{n r}=\int_{-\theta_{\mathrm{p}}}^{\theta_{\mathrm{p}}} \cos (r \theta) \cos (n \theta) d \theta \\
\left.I_{n r}=\left\{\begin{array}{c}
\frac{1}{4 n}\left[4 n \theta_{\mathrm{p}}+2 \sin \left(2 n \theta_{\mathrm{p}}\right)\right], n=r \\
\frac{1}{r^{2}-n^{2}}\left[-n \cos \left(r \theta_{\mathrm{p}}\right) \sin \left(n \theta_{\mathrm{p}}\right)+r \cos \left(n \theta_{\mathrm{p}}\right) \sin \left(r \theta_{\mathrm{p}}\right)-\right. \\
-n \cos \left(r \theta_{\mathrm{p}}\right) \sin \left(n \theta_{\mathrm{p}}\right)+r \cos \left(n \theta_{\mathrm{p}}\right) \sin \left(r \theta_{\mathrm{p}}\right) \\
2 \theta_{\mathrm{p}}, n=r=0
\end{array}\right\} r+n\right\}
\end{gathered}
$$

Therefore, the equation for the unknown coefficients $W_{s n}$ becomes 


$$
\sum_{n=0}^{N} I_{n r} W_{s n}^{(3)}=\frac{2}{s \pi}\left[1-(-1)^{s}\right] \cdot\left\{[\bar{h}-a] I_{r}^{(1)}-a I_{r}^{(2)}\right\}
$$

with

$$
\begin{aligned}
& r=0,1,2, \ldots, R \\
& n=0,1,2, \ldots, N \\
& s=0,1,2, \ldots, S
\end{aligned}
$$

Calling

$$
f_{s r}=\frac{2}{s \pi}\left[1-(-1)^{s}\right] \cdot\left\{[\bar{h}-a] I_{r}^{(1)}-a I_{r}^{(2)}\right\}
$$

Equation (B.8) becomes

$$
\sum_{n=0}^{N} I_{n r} W_{s n}^{(3)}=f_{s r}
$$

Or in matrix notation

$$
\left[I_{n r}(\bar{h})\right]\left\{W_{s n}^{(3)}\right\}=\left\{f_{s r}(\bar{h})\right\}
$$

Equation (B.11) is repeated in Chapter 2 as equation (2.31) in order to continue the analytical development. 


\section{APPENDIX C: GENERAL BOUNDARY FORCES FOR A SHELL}

This appendix present the calculation of the boundary forces for a general elastic shell. The forces derived in this appendix were then used in the solving of the transmitted forces to the vehicle spindle.

The stress field in a differential piece of shell material is shown in Figure C.1. They are

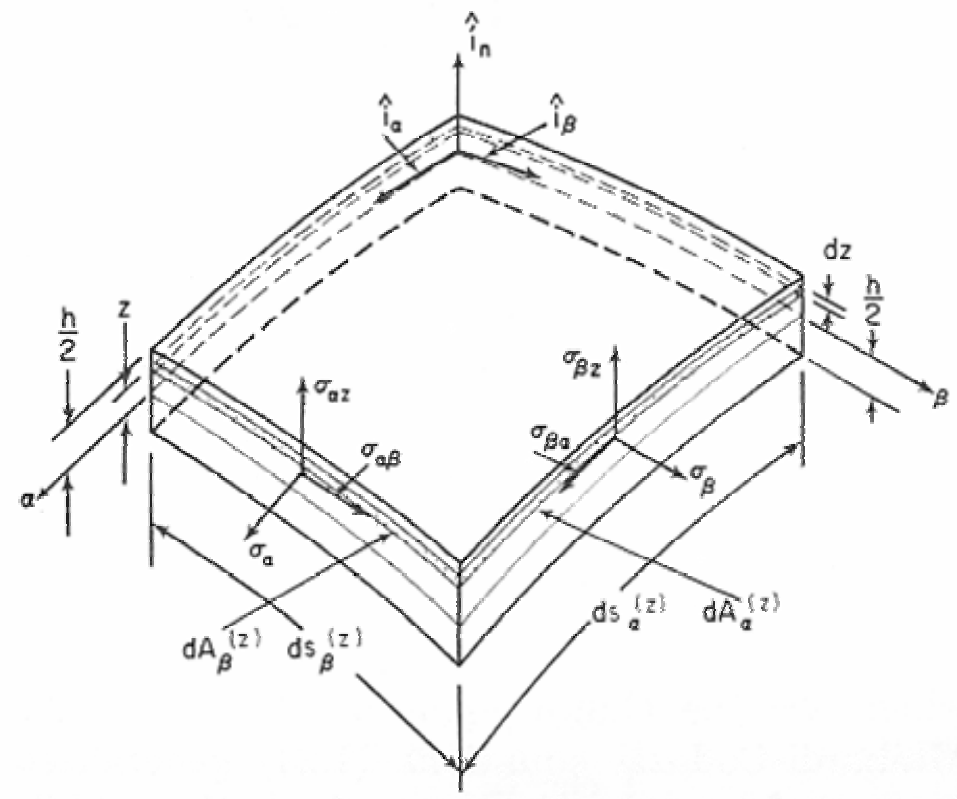

Figure C.1: Stresses in shell coordinates (Leissa, 1993).

$$
\begin{gathered}
\sigma_{\alpha}=\frac{E}{1-v^{2}}\left(\varepsilon_{\alpha}+v \varepsilon_{\beta}\right) \\
\sigma_{\beta}=\frac{E}{1-v^{2}}\left(\varepsilon_{\beta}+v \varepsilon_{\alpha}\right) \\
\sigma_{\alpha \beta}=\frac{E}{2(1+v)} \gamma_{\alpha \beta}
\end{gathered}
$$


The coefficients $\varepsilon_{\alpha}, \varepsilon_{\beta}$, and $\gamma_{\alpha \beta}$ are the total strain according to the Donnel-MushtariVlasov operator(Leissa, 1993). The resulting forces per unit length in the face perpendicular to $\alpha$ - axis are given by

$$
\begin{aligned}
& N_{\alpha}=\int_{-h / 2}^{h / 2} \sigma_{\alpha}\left(1+\frac{z}{R_{\beta}}\right) d z \\
& N_{\alpha \beta}=\int_{-h / 2}^{h / 2} \sigma_{\alpha \beta}\left(1+\frac{Z}{R_{\beta}}\right) d z \\
& Q_{\alpha}=\int_{-h / 2}^{h / 2} \sigma_{\alpha z}\left(1+\frac{z}{R_{\beta}}\right) d z
\end{aligned}
$$

and similarly the force resultants on the face perpendicular to the $\beta$ - axis are given by

$$
\begin{aligned}
N_{\beta} & =\int_{-h / 2}^{h / 2} \sigma_{\beta}\left(1+\frac{z}{R_{\alpha}}\right) d z \\
N_{\beta \alpha} & =\int_{-h / 2}^{h / 2} \sigma_{\beta \alpha}\left(1+\frac{z}{R_{\alpha}}\right) d z \\
Q_{\beta} & =\int_{-h / 2}^{h / 2} \sigma_{\beta z}\left(1+\frac{z}{R_{\alpha}}\right) d z
\end{aligned}
$$

The positive direction of the force resultant forces are shown in Figure C.2. 


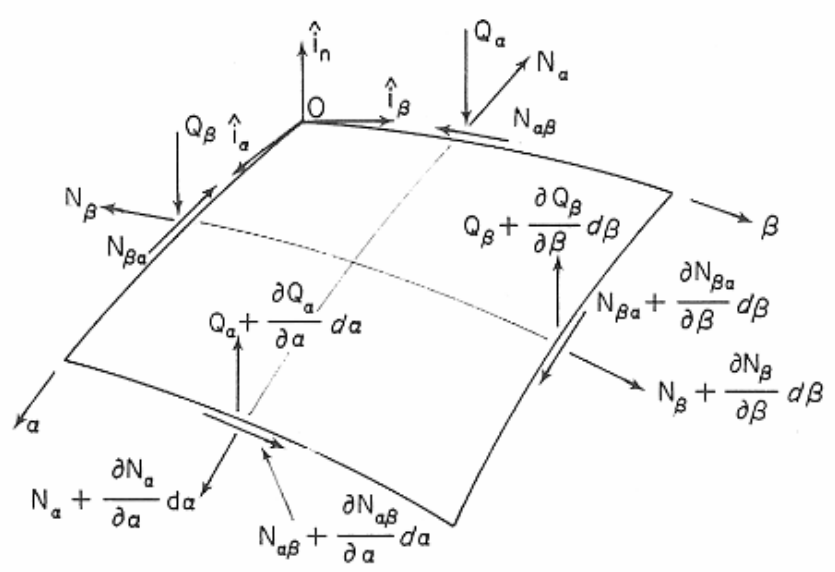

Figure C.2: Force resultants in shell coordinates (Leissa, 1993).

The moment of the infinitesimal force $\sigma_{\alpha} d s_{\beta}^{(z)} d z$ about the $\beta$-line is given by $z \sigma_{\alpha} d s_{\beta}^{(z)} d z$. This moment has the unit of moment per unit length

$$
M_{\alpha}=\int_{-h / 2}^{h / 2} \sigma_{\alpha}\left(1+\frac{z}{R_{\beta}}\right) z d z
$$

and

$$
M_{\alpha \beta}=\int_{-h / 2}^{h / 2} \sigma_{\alpha \beta}\left(1+\frac{z}{R_{\beta}}\right) z d z
$$

On the other face the resultant moments are given

$$
M_{\beta}=\int_{-h / 2}^{h / 2} \sigma_{\beta}\left(1+\frac{Z}{R_{\alpha}}\right) z d z
$$

and 


$$
M_{\beta \alpha}=\int_{-h / 2}^{h / 2} \sigma_{\beta \alpha}\left(1+\frac{z}{R_{\alpha}}\right) z d z
$$

The positive direction of the moment is shown in Figure C.3. Note that although the stress tensor in the three-dimensional space is symmetric $\sigma_{\alpha \beta}=\sigma_{\beta \alpha}$, is clear from equations (C.5), (C.8), (C.11), and (C.13) that $N_{\alpha \beta} \neq N_{\beta \alpha}$ and $M_{\alpha \beta} \neq M_{\beta \alpha}$ unless $R_{\alpha}=R_{\beta}$.

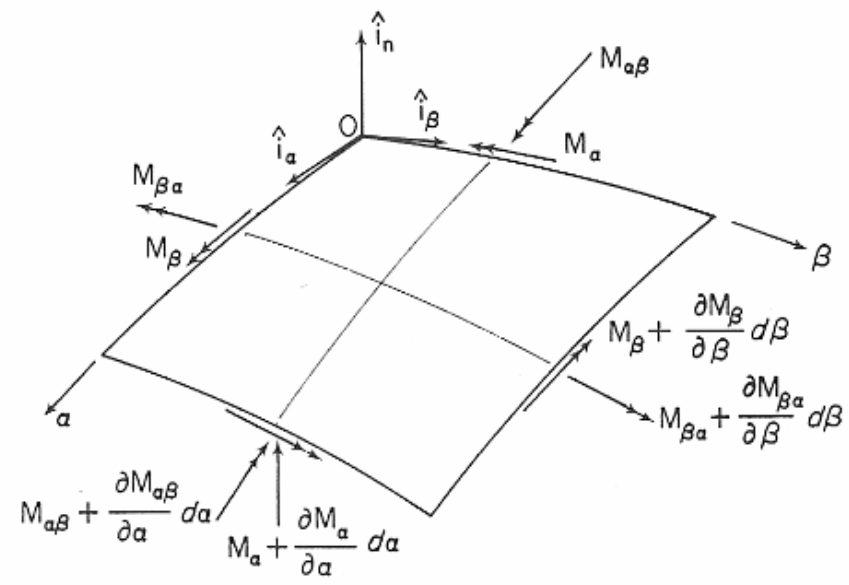

Figure C.3: Moment resultants in shell coordinates (Leissa, 1993).

Assuming that the boundaries lie along the coordinate curves, i.e. Figure C.1, the virtual work done by the reaction forces at the boundary is zero.

$$
W_{1}=\left.\int_{\beta_{1}}^{\beta_{2}}\left(\vec{F}_{\alpha} \cdot \vec{u}+\vec{M}_{\alpha} \cdot \vec{\Omega}\right)\right|_{\alpha=\alpha_{2}} B d \beta=0
$$

along the boundary $\alpha=\alpha_{2}$ and

$$
W_{2}=\left.\int_{\alpha_{1}}^{\alpha_{2}}\left(\vec{F}_{\beta} \cdot \vec{u}+\vec{M}_{\beta} \cdot \vec{\Omega}\right)\right|_{\beta=\beta_{2}} A d \alpha=0
$$


along the boundary $\beta=\beta_{2}$. The forces $\vec{F}_{\alpha}, \vec{F}_{\beta}$ and the moments $\vec{M}_{\alpha}, \vec{M}_{\beta}$ are given by the equations

$$
\begin{aligned}
& \vec{F}_{\alpha}=\left(N_{\alpha} i_{\llcorner\alpha}+N_{\alpha \beta} i_{\beta}+Q_{\alpha} i_{n}\right) B d \beta \\
& \vec{F}_{\beta}=\left(N_{\beta \alpha} i_{\alpha}+N_{\beta} i_{\beta}+Q_{\beta} i_{\mathfrak{N}}\right) A d \beta \\
& \vec{M}_{\alpha}=\left(-M_{\alpha \beta} i_{\alpha}+M_{\alpha} i_{\beta}\right) B d \beta \\
& \vec{M}_{\beta}=\left(-M_{\beta} i_{\alpha \alpha}+M_{\beta \alpha} i_{\alpha \beta}\right) B d \beta
\end{aligned}
$$

and

$$
\begin{aligned}
& \vec{u}=u i_{\sim \alpha}+v i_{\beta}+w i_{\sim} \\
& \vec{\Omega}=-\theta_{\beta} i_{\alpha}+\theta_{\alpha} i_{\beta}
\end{aligned}
$$

where

$$
\begin{aligned}
& \theta_{\alpha}=\frac{u}{R_{\alpha}}-\frac{1}{A} \frac{\partial w}{\partial \alpha} \\
& \theta_{\beta}=\frac{v}{R_{\beta}}-\frac{1}{B} \frac{\partial w}{\partial \beta}
\end{aligned}
$$

Note that $A$ and $B$ are de length of the tangent vectors to the $\alpha$ and $\beta$ curves respectively, and $\frac{1}{R_{\alpha}}$ and $\frac{1}{R_{\beta}}$ are the curvatures of the $\alpha$ and $\beta$ curves. These parameters satisfying the so-called Gauss ${ }^{1}$-Mainardi-Codazzi relations determine the shell surface uniquely, except to position and orientation in space, Leissa (1993).

\footnotetext{
${ }^{1}$ Carl Friedrich Gauss

Born: April 30, 1777, Brunswick, Germany.

Died: February 23, 1855, Göttingen, Germany.

From the outside, Gauss' life was very simple. Having brought up in an austere childhood in a poor and uneducated family he showed extraordinary precocity. He received a stipend from the duke of Brunswick starting at the age of 14 which allowed him to devote his time to his studies for 16 years. Before his 25th birthday, he was already famous for his work in mathematics and astronomy. When he became 30 he went to Göttingen to become director of the observatory. He rarely left the city except on scientific business. From there, he worked for 47 years until his death at almost 78. In contrast to his external simplicity, Gauss' personal life was tragic and complicated. Due to the French Revolution, Napoleonic period and the democratic revolutions in Germany, he suffered from political turmoil and financial insecurity. He found no fellow mathematical collaborators and worked alone for most of his life. An unsympathetic father, the
} 
Replacing equations (C.17) and (C.16) into (C.14) and (C.15), it gives

$$
W_{1}=\left.\int_{\beta_{1}}^{\beta_{2}}\left(N_{\alpha} u+N_{\alpha \beta} v+Q_{\alpha} w+M_{\alpha \beta} \theta_{\beta}+M_{\alpha} \theta_{\alpha}\right)\right|_{\alpha=\alpha_{2}} B d \beta=0
$$

and

$$
W_{2}=\left.\int_{\alpha_{1}}^{\alpha_{2}}\left(N_{\alpha \beta} u+N_{\beta} v+Q_{\beta} w+M_{\beta} \theta_{\beta}+M_{\beta \alpha} \theta_{\alpha}\right)\right|_{\beta=\beta_{2}} A d \alpha=0
$$

by using equation (C.18), equations (C.19) and (C.20) becomes

$$
W_{1}=\left.\int_{\beta_{1}}^{\beta_{2}}\left[N_{\alpha} u+N_{\alpha \beta} v+Q_{\alpha} w+M_{\alpha \beta}\left(\frac{v}{R_{\beta}}-\frac{1}{B} \frac{\partial w}{\partial \beta}\right)+M_{\alpha} \theta_{\alpha}\right]\right|_{\alpha=\alpha_{2}} B d \beta=0
$$

and

$$
W_{2}=\left.\int_{\alpha_{1}}^{\alpha_{2}}\left[N_{\alpha \beta} u+N_{\beta} v+Q_{\beta} w+M_{\beta} \theta_{\beta}+M_{\beta \alpha}\left(\frac{u}{R_{\alpha}}-\frac{1}{A} \frac{\partial w}{\partial \alpha}\right)\right]\right|_{\beta=\beta_{2}} A d \alpha=0
$$

Integrating by parts the following equations, it gives

$$
\int_{\beta_{1}}^{\beta_{2}} M_{\alpha \beta} \frac{1}{B} \frac{\partial w}{\partial \beta} B d \beta=\left.M_{\alpha \beta} w\right|_{\beta_{1}} ^{\beta_{2}}-\int_{\beta_{1}}^{\beta_{2}} \frac{\partial M_{\alpha \beta}}{\partial \beta} w d \beta
$$

and

$$
\int_{\alpha_{1}}^{\alpha_{2}} M_{\beta \alpha} \frac{1}{A} \frac{\partial w}{\partial \alpha} A d \alpha=\left.M_{\beta \alpha} w\right|_{\alpha_{1}} ^{\alpha_{2}}-\int_{\beta_{1}}^{\beta_{2}} \frac{\partial M_{\beta \alpha}}{\partial \alpha} w d \alpha
$$

Substituting equations (C.23) and (C.24) into (C.21) and (C.22) respectively, it yields

early death of his first wife, the poor health of his second wife, and terrible relations with his sons denied him a family sanctuary until late in life.

Even with all of these troubles, Gauss kept an amazingly rich scientific activity. An early passion for numbers and calculations extended first to the theory of numbers, to algebra, analysis, geometry, probability, and the theory of errors. At the same time, he carried on intensive empirical and theoretical research in many branches of science, including observational astronomy, celestial mechanics, surveying, geodesy, capillarity, geomagnetism, electromagnetism, mechanism optics, actuarial science. His publications, abundant correspondence, notes, and manuscripts show him to have been one of the greatest scientific virtuosos of all time.

(Source: http://www.geocities.com/RainForest/Vines/2977/gauss/english.html ) 


$$
\begin{aligned}
W_{1}= & \left.\int_{\beta_{1}}^{\beta_{2}}\left[N_{\alpha} u+\left(N_{\alpha \beta}+\frac{M_{\alpha \beta}}{R_{\beta}}\right) v+\left(Q_{\alpha}+\frac{1}{B} \frac{\partial M_{\alpha \beta}}{\partial \beta}\right) w+M_{\alpha} \theta_{\alpha}\right]\right|_{\alpha=\alpha_{2}} B d \beta \\
& -\left.M_{\alpha \beta} w\right|_{\beta_{1}, \alpha=\alpha_{2}} ^{\beta_{2}}=0
\end{aligned}
$$

and

$$
\begin{aligned}
W_{2}= & \left.\int_{\alpha_{1}}^{\alpha_{2}}\left[\left(N_{\beta \alpha}+\frac{M_{\beta \alpha}}{R_{\alpha}}\right) u+N_{\beta} v+\left(Q_{\beta}+\frac{1}{A} \frac{\partial M_{\beta \alpha}}{\partial \alpha}\right) w+M_{\beta} \theta_{\beta}\right]\right|_{\beta=\beta_{2}} A d \alpha \\
& -\left.M_{\beta \alpha} w\right|_{\alpha_{1}, \beta=\beta_{2}} ^{\alpha_{2}}=0
\end{aligned}
$$

Equations (C.25) and (C.26) are satisfied if the integrand and the second parts of the equation are set equal to zero. The boundary conditions for the shell where $\alpha=$ constant are

$$
\begin{array}{ccc}
N_{\alpha} & \text { or } & u=0 \\
N_{\alpha \beta}+\frac{M_{\alpha \beta}}{R_{\beta}} & \text { or } & v=0 \\
Q_{\alpha}+\frac{1}{B} \frac{\partial M_{\alpha \beta}}{\partial \beta} & \text { or } & w=0 \\
M_{\alpha} & \text { or } & \theta_{\alpha}=0 \\
\left.M_{\alpha \beta} w\right|_{\beta_{1}} ^{\beta_{2}}=0 & &
\end{array}
$$

and on the edge where $\beta=$ constant

$$
\begin{array}{rrrr}
N_{\beta \alpha}+\frac{M_{\beta \alpha}}{R_{\alpha}} & \text { or } & u=0 \\
N_{\beta} & \text { or } & v=0 \\
Q_{\beta}+\frac{1}{A} \frac{\partial M_{\beta \alpha}}{\partial \alpha} & \text { or } & w=0 \\
M_{\beta} & \text { or } & \theta_{\beta}=0 \\
\left.M_{\beta \alpha} w\right|_{\alpha_{1}} ^{\alpha_{2}}=0 & &
\end{array}
$$




\section{APPENDIX D: SOLVING THE SPECTRAL PROPERTIES OF THE HELMHOLTZ OPERATOR}

The Helmholtz equation is given by

$$
\nabla^{2} \Psi+k^{2} \Psi=0
$$

that in cylindrical coordinates is given as,

$$
\frac{d^{2} \Psi}{d r^{2}}+\frac{1}{r} \frac{d \Psi}{d r}+\frac{1}{r^{2}} \frac{d^{2} \Psi}{d \theta^{2}}+\frac{d^{2} \Psi}{d x^{2}}+k^{2} p=0
$$

The rigid wall boundary conditions are defined as follow

$$
\left(\frac{d \Psi}{d x}\right)_{x=0}=\left(\frac{d \Psi}{d x}\right)_{x=L_{T}}=\left(\frac{d \Psi}{d r}\right)_{r=a}=\left(\frac{d \Psi}{d r}\right)_{r=b}=0
$$

The solution is assumed to have the form

$$
\Psi(x, \theta, r)=R(r) \Theta(\theta) X(x)
$$

Due to the assumed form of the solution, the separation of variables technique is applied. This technique leads to a set of three differential systems. The first system shown in the equation (D.5) is related to the axial direction. 


$$
\begin{aligned}
& \frac{1}{X(x)} \frac{d^{2} X(x)}{d x^{2}}=k_{x q}^{2} \\
& \text { with } \\
& \frac{d X}{d x}(0)=0 \text { and } \frac{d X}{d x}\left(L_{T}\right)=0
\end{aligned}
$$

For the differential system given by (D.5) related to the axial direction the spectral properties are given by

$$
\begin{aligned}
& k_{x q}^{2}=\frac{q^{2} \pi^{2}}{L_{T}^{2}} ; \text { with } q=0,1, \ldots, Q \\
& \text { and } \\
& X_{q}(x)=A \cos \left(\frac{q \pi}{L_{T}} x\right) \text {, with } 0<\mathrm{x}<\mathrm{L}_{\mathrm{T}}
\end{aligned}
$$

The value $A \neq 0$ included in the Real domain is not defined in the eigenfunction $X_{q}(x)$. The reason is that there is an infinite set of eigenfunction $X_{q}(x)$ defined for each value of $A$. Each eigenfunction $X_{q}(x)$ satisfies the system given by equation (D.5) therefore for simplicity the value $A=1$ is assumed.

The second system given by (D.7) is related to the azimuthal direction

$$
\begin{aligned}
& \frac{1}{\Theta(\theta)} \frac{d^{2} \Theta(\theta)}{d \theta^{2}}=-p^{2} \\
& \text { with } \\
& \Theta(\theta)=\Theta(\theta+2 \pi)
\end{aligned}
$$


The condition $\Theta(\theta)=\Theta(\theta+2 \pi)$ implies a periodic solution of the differential equation in the domain $0 \leq \theta<2 \pi$. The third and last system is related to the radial direction of the acoustic cavity. The spectral properties are given by

$$
\begin{aligned}
& p=0,1, \ldots, P \\
& \text { and } \\
& \Theta_{p}(\theta)=B \cos (p \theta+\phi)
\end{aligned}
$$

The value of $B$ in the eigenfunction $\Theta_{p}(\theta)$ is assumed to be 1 due to the same justification than in (D.7). The "orientation" or phase angle of $\Theta_{p}(\theta)$ is given by $\phi$, which is not defined by the system (D.7). The value of $\phi$ is defined by the external forcing function acting upon the system. Finally, the third system associated to the radial direction is given by

$$
\begin{aligned}
& r^{2} \frac{d^{2} R(r)}{d r^{2}}+r \frac{d R(r)}{d r}+R(r)\left(r^{2} k_{p l}^{2}-p^{2}\right)=0 \\
& \text { with } \\
& \frac{d R(a)}{d r}=\frac{d R(b)}{d r}=0
\end{aligned}
$$

The equation given by (D.9) has the form of the Bessel equation, whose general solution is known. The general solution is given in term of Bessel functions as follow

$$
R(r)=A_{p} J_{p}\left(k_{p l} r\right)+B_{p} Y_{p}\left(k_{p l} r\right)
$$

where $J_{p}(\cdot)$ is the Bessel's function of first kind and $Y_{p}(\cdot)$ is the Bessel's function of second kind. Replacing equation (D.10) into (D.9), the algebra leads to the characteristic equation

$$
J_{p}^{\prime}\left(k_{p l} a\right) Y_{p}^{\prime}\left(k_{p l} b\right)-Y_{p}^{\prime}\left(k_{p l} a\right) J_{p}^{\prime}\left(k_{p l} b\right)=0
$$


The roots of this equation $k_{p l}$ must be obtained numerically. However, for the case of $b>0.5 a$ the characteristic equation was solved approximately by Blevins (1995) with the following result

$$
k_{p l} \cong \sqrt{\left(\frac{l \pi}{a-b}\right)^{2}+\left(\frac{2 p}{a+b}\right)^{2}}
$$

The values of $A_{p}$ and $B_{p}$ are given by

$$
A_{p}=Y_{p}^{\prime}\left(k_{p l} a\right) \text { and } B_{p}=-J_{p}^{\prime}\left(k_{p l} a\right)
$$

Therefore, the spectral properties of the acoustic cavity are obtained by combining equations (D.6), (D.8), (D.12), and (D.10).The eigenvalue is then

$$
k_{q p l}=\frac{\omega_{q p l}}{c}=\frac{2 \pi f_{q p l}}{c} \cong \sqrt{\left(\frac{l \pi}{a-b}\right)^{2}+\left(\frac{2 p}{a+b}\right)^{2}+\left(\frac{q \pi}{L_{T}}\right)^{2}}
$$

and the mode shape is

$$
\begin{array}{r}
\Psi_{q p l}(x, \theta, r)=\rho c^{2}\left[A_{p} J_{p}\left(k_{p l} r\right)+B_{p} Y_{p}\left(k_{p l} r\right)\right] \cos (p \theta) \cos \left(k_{x q} x\right) \\
\text { with } q, p, l=0,1,2, \ldots
\end{array}
$$

Orthogonality condition of the acoustic modes leads to

$$
\int_{0}^{2 \pi} \int_{0}^{L_{T}} \int_{b}^{a} \Psi_{q p l}(x, \theta, r) \Psi_{r s o}(x, \theta, r) r d r d x d \theta=\Lambda_{q p l} \delta_{q r} \delta_{p s} \delta_{l o}
$$




\section{APPENDiX E: THE COUPLING MATRIX}

Especial attention will be given to the obtaining of the coupling matrix $\sum_{q=0}^{Q} \sum_{p=0}^{P} \sum_{l=0}^{L}\left[\alpha_{s r m n q p}\right]$.

The coupling matrix is obtained from the following integration scheme

$$
\int_{0}^{L_{r}} \int_{0}^{2 \pi}\left[\Phi_{r s}\right]^{T}\left\{\begin{array}{c}
0 \\
0 \\
p(x, \theta, a)
\end{array}\right\} a d \theta d x
$$

where

$$
\begin{gathered}
p(x, \theta, a)=\int_{0}^{L_{r}} \int_{0}^{2 \pi} i \omega \rho_{A} i \omega \sum_{m=1}^{M} \sum_{n=0}^{N}\left\{W_{m n}^{(1)}, W_{m n}^{(2)}, \quad W_{m n}^{(3)}\right\}\left\{\begin{array}{c}
A_{m n}^{(1)} \\
A_{m m}^{(2)} \\
A_{m n}^{(3)}
\end{array}\right\} \sin \left(\frac{m \pi}{L_{T}} x_{o}\right) \cos \left(n \theta_{o}\right) . \\
\cdot \sum_{q=0}^{Q} \sum_{p=0}^{P} \sum_{l=0}^{L} \frac{\Psi_{q p l}(x, \theta, a) \Psi_{q p l}\left(x_{o}, \theta_{o}, a\right)}{\Lambda_{q p l}\left(k_{q p l}^{2}-k^{2}\right)} a d \theta_{o} d x_{o}
\end{gathered}
$$

and

$$
\begin{gathered}
\Psi_{q p l}(r, \theta, x)=\rho c^{2}\left[Y_{p}^{\prime}\left(k_{p l} a\right) J_{p}\left(k_{p l} r\right)-J_{p}^{\prime}\left(k_{p l} a\right) Y_{p}\left(k_{p l} r\right)\right] \cos (p \theta) \cos \left(k_{x q} x\right) \\
\text { with } q, p, l=0,1,2, \ldots \\
{\left[\Phi_{r s}\right]^{T}=\left[\begin{array}{lll}
U_{r s}^{(1)} \cos (r \theta) \cos \left(\lambda_{s} \frac{x}{a}\right) & V_{r s}^{(1)} \sin (r \theta) \sin \left(\lambda_{s} \frac{x}{a}\right) & W_{r s}^{(1)} \cos (r \theta) \sin \left(\lambda_{s} \frac{x}{a}\right) \\
U_{r s}^{(2)} \cos (r \theta) \cos \left(\lambda_{s} \frac{x}{a}\right) & V_{r s}^{(2)} \sin (r \theta) \sin \left(\lambda_{s} \frac{x}{a}\right) & W_{r s}^{(2)} \cos (r \theta) \sin \left(\lambda_{s} \frac{x}{a}\right) \\
U_{r s}^{(3)} \cos (r \theta) \cos \left(\lambda_{s} \frac{x}{a}\right) & V_{r s}^{(3)} \sin (r \theta) \sin \left(\lambda_{s} \frac{x}{a}\right) & W_{r s}^{(3)} \cos (r \theta) \sin \left(\lambda_{s} \frac{x}{a}\right)
\end{array}\right]}
\end{gathered}
$$

recall that $\lambda_{s}=\frac{s \pi a}{L_{T}}$. Then, equation (E.1) becomes, 


$$
\begin{gathered}
\int_{0}^{L_{\mathrm{r}}} \int_{0}^{2 \pi}\left[\Phi_{r s}\right]^{T}\left\{\begin{array}{c}
0 \\
0 \\
p(x, \theta, a)
\end{array}\right\} a d \theta d x=\int_{0}^{L_{r}} \int_{0}^{2 \pi}\left[\int_{0}^{L_{r}} \int_{0}^{2 \pi}\left\{\begin{array}{l}
W_{r s}^{(1)} \\
W_{r s}^{(2)} \\
W_{r s}^{(3)}
\end{array}\right\} \cos (r \theta) \sin \left(\frac{s \pi}{L_{T}} x\right) i \omega \rho_{A} i \omega \sum_{m=1}^{M} \sum_{n=0}^{N}\left\{W_{m n}^{(1)}, \quad W_{m n}^{(2)}, \quad W_{m n}^{(3)}\right\} .\right. \\
\left\{\begin{array}{c}
A_{m n}^{(1)} \\
A_{m n}^{(2)} \\
A_{m n}^{(3)}
\end{array}\right\} \sin \left(\frac{m \pi}{L_{T}} x_{o}\right) \cos \left(n \theta_{o}\right) \sum_{q=0}^{Q} \sum_{p=0}^{P} \sum_{l=0}^{L} \frac{\left[Y_{p}^{\prime}\left(k_{p l} a\right) J_{p}\left(k_{p l} a\right)-J_{p}^{\prime}\left(k_{p l} a\right) Y_{p}\left(k_{p l} a\right)\right] \cos (p \theta) \cos \left(k_{x q} x\right)}{\Lambda_{q p l}\left(k_{q p l}^{2}-k^{2}\right)} \\
\left.\cdot\left[Y_{p}^{\prime}\left(k_{p l} a\right) J_{p}\left(k_{p l} a\right)-J_{p}^{\prime}\left(k_{p l} a\right) Y_{p}\left(k_{p l} a\right)\right] \cos \left(p \theta_{o}\right) \cos \left(k_{x q} x_{o}\right) d \theta_{o} d x_{o}\right] a d \theta d x
\end{gathered}
$$

Performing the integration, equation (E.5) becomes

$$
\begin{gathered}
\int_{0}^{L_{r}} \int_{0}^{2 \pi}\left[\Phi_{r s}\right]^{T}\left\{\begin{array}{c}
0 \\
0 \\
p(x, \theta, a)
\end{array}\right\} d \theta d x=-\omega^{2} \rho_{A} \pi^{2} \sum_{m=1}^{M} \sum_{n=0}^{N} \sum_{q=0}^{Q} \sum_{p=0}^{p} \sum_{l=0}^{L} \frac{\left[Y_{p}^{\prime}\left(k_{p l} a\right) J_{p}\left(k_{p l} a\right)-J_{p}^{\prime}\left(k_{p l} a\right) Y_{p}\left(k_{p l} a\right)\right]^{2}}{\Lambda_{q p l}\left(k_{q p l}^{2}-k^{2}\right)} \\
\delta_{r p} \delta_{n p} \varepsilon_{r} \varepsilon_{n} \varepsilon_{s q} \varepsilon_{m q}\left[\begin{array}{lll}
W_{r s}^{(1)} W_{m n}^{(1)} & W_{r s}^{(1)} W_{m n}^{(2)} & W_{r s}^{(1)} W_{m n}^{(3)} \\
W_{r s}^{(2)} W_{m n}^{(1)} & W_{r s}^{(2)} W_{m n}^{(2)} & W_{r s}^{(2)} W_{m n}^{(3)} \\
W_{r s}^{(3)} W_{m n}^{(1)} & W_{r s}^{(3)} W_{m n}^{(2)} & W_{r s}^{(3)} W_{m n}^{(3)}
\end{array}\right]\left\{\begin{array}{c}
A_{m n}^{(1)} \\
A_{m n}^{(2)} \\
A_{m n}^{(3)}
\end{array}\right\}
\end{gathered}
$$

where

$$
\begin{aligned}
& \varepsilon_{r}= \begin{cases}2, & r=0 \\
1, & r \neq 0\end{cases} \\
& \varepsilon_{n}= \begin{cases}2, & n=0 \\
1, & n \neq 0\end{cases} \\
& \varepsilon_{s q}= \begin{cases}0, & q=s \\
\frac{\left[-1+(-1)^{q+s}\right] L s}{\pi\left(q^{2}-s^{2}\right)}, & q \neq s\end{cases} \\
& \varepsilon_{m q}= \begin{cases}0, & m=q \\
\frac{\left[-1+(-1)^{q+m}\right] L m}{\pi\left(q^{2}-m^{2}\right)}, & m \neq q\end{cases}
\end{aligned}
$$

Equation (E.6) can be written as

$$
\int_{0}^{L_{T}} \int_{0}^{2 \pi}\left[\Phi_{r s}\right]^{T}\left\{\begin{array}{c}
0 \\
0 \\
p(x, \theta, a)
\end{array}\right\} d \theta d x=\Omega^{2} \sum_{q=0}^{Q} \sum_{p=0}^{P} \sum_{l=0}^{L}\left[\alpha_{\text {srmnqpl }}\right]\left\{A_{m n}\right\}
$$

In matrix notation equation (E.8) becomes 


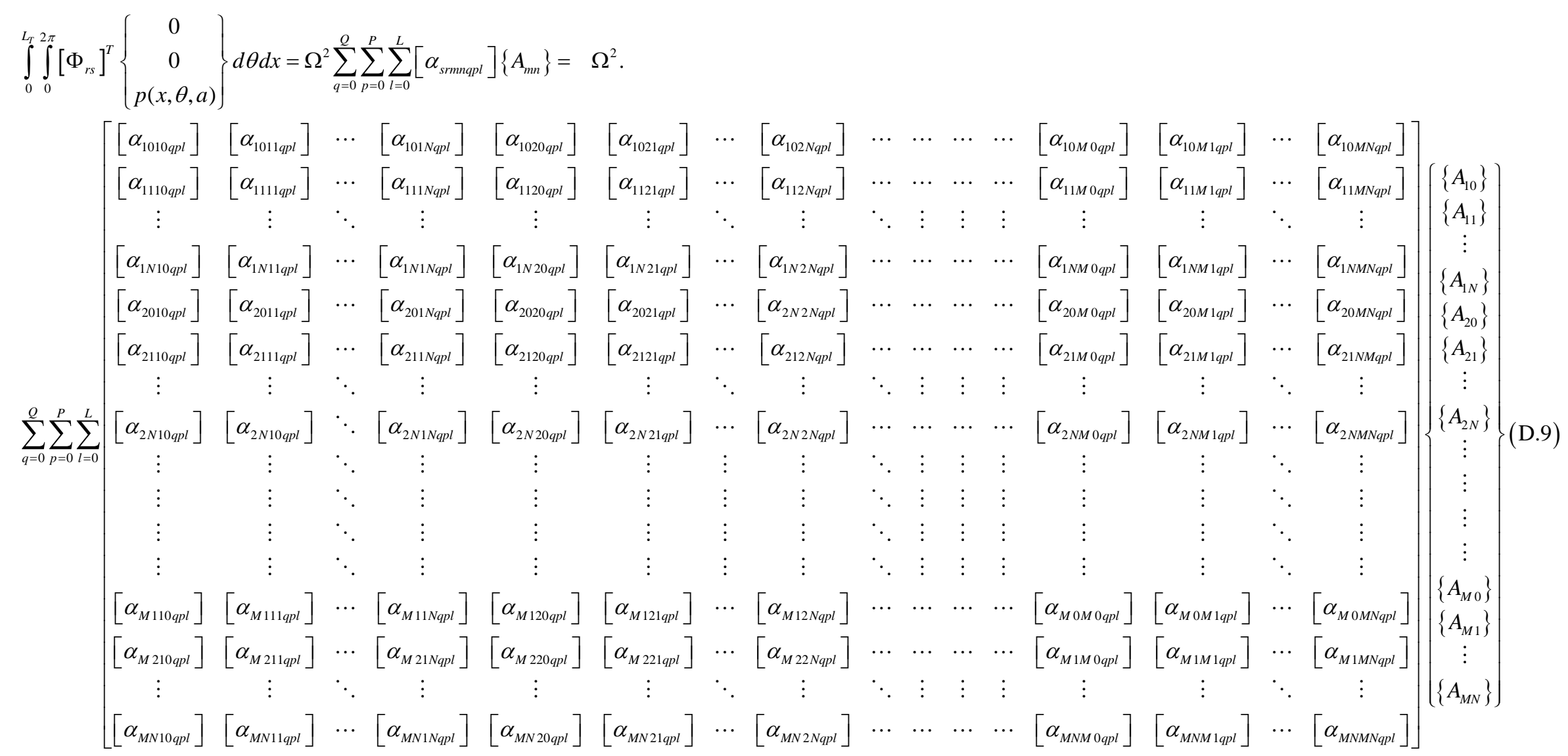




\section{APPENDIX F: SCREEN ACOUSTIC IMPEDANCE MODEL}

In this appendix the model of the acoustic impedance $Z_{S}^{(j)}$ of the screen placed at the coupling interface is presented. The model presented here is based on the work by Ingard and Ising (1967).

The normalized linear impedance $Z_{S}^{(j)}$ is given by

$$
\frac{Z_{S}^{(j)}}{\rho_{A} c}=\frac{\sqrt{8 \mu \rho_{A} \omega}}{\rho_{A} c \sigma}\left(1+\frac{t}{d}\right)+\frac{i \omega}{c \sigma}\left(t+\frac{8 d}{3 \pi}\right)
$$

The parameters of the perforate screen (facesheet) are as follows:

$$
\begin{aligned}
& t=\text { thickness of the perforate screen (facesheet) }[\mathrm{m}] \\
& r=\text { radius of orifices } \\
& d=\text { diameter of orifices }(d=2 r) \\
& \sigma=\text { screen (facesheet) porosity or percentage open area (POA) }
\end{aligned}
$$

while the properties of the fluid are given by

$$
\begin{aligned}
& c=\text { speed of sound }(343 \mathrm{~m} / \mathrm{s}) \\
& \rho_{A}=\text { density of air }\left(\sim 1.21 \mathrm{NS}^{2} / \mathrm{m}^{4} \text { at } 20{ }^{\circ} \mathrm{C}\right) \\
& \mu=\text { viscosity of air }\left(\sim 18 \times 10^{-6} \mathrm{NS} / \mathrm{m} \text { at } 20{ }^{\circ} \mathrm{C}\right)
\end{aligned}
$$

The screen normalized acoustic impedance is evaluated numerically for a thickness of the perforated screen $t=0.00075 \mathrm{~m}$, radius of the orifices $r=0.00075 \mathrm{~m}$, and a screen porosity $\sigma=0.05($ POA 5\%). 
The resistive part of the normalized linear impedance $Z_{s}^{(j)}$ is shown in Figure F.1 and the reactive part in Figure F.2. The reactive component of the acoustic impedance shows a nonlinear behavior at low frequency $(<130 \mathrm{~Hz})$. For higher frequencies $(>130 \mathrm{~Hz})$ the resistivity can be considered linear. The reactive component is linear in the complete frequency range.

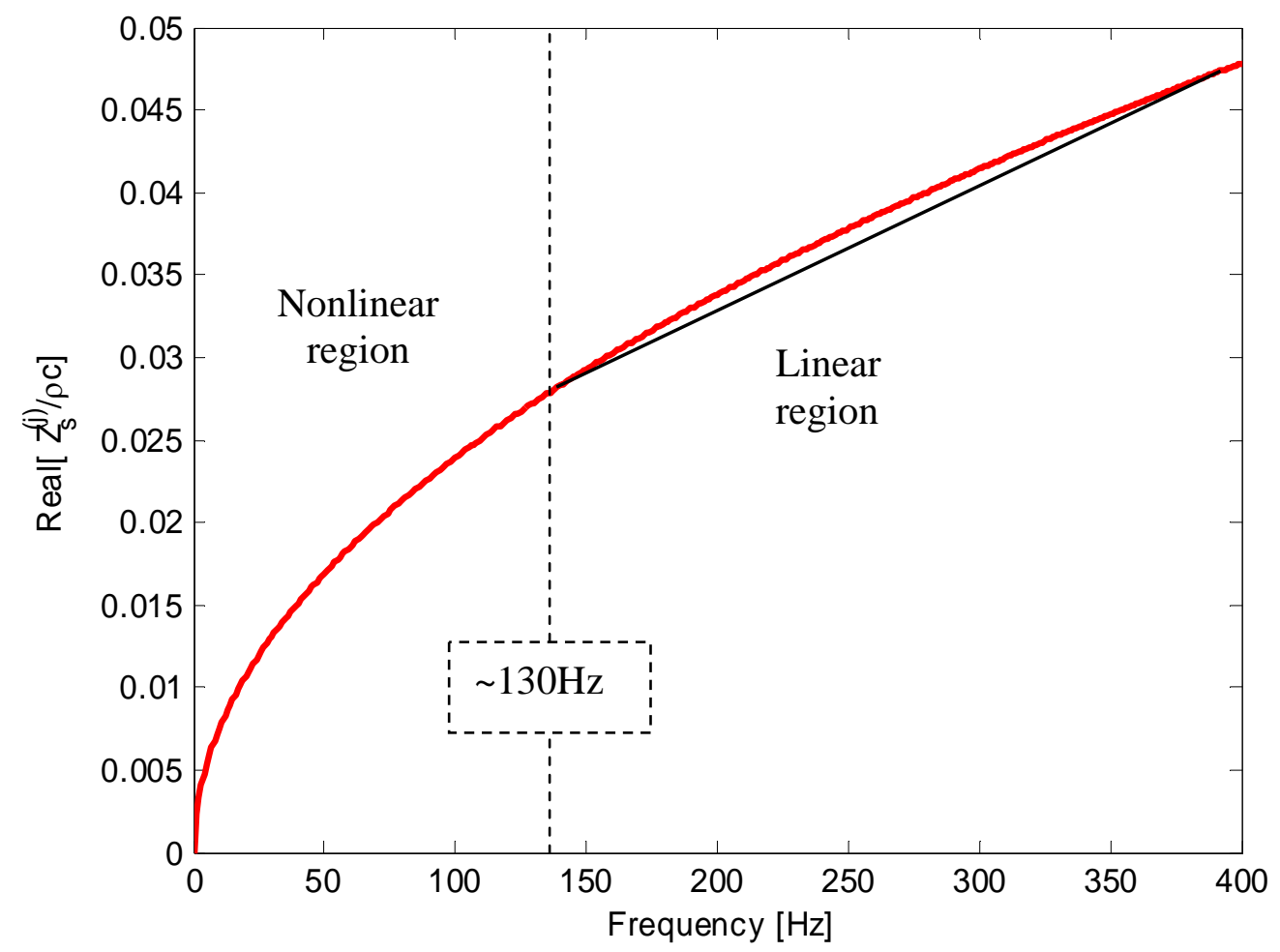

Figure F.1: Facesheet Normalized Impedance - Resistive Component. 


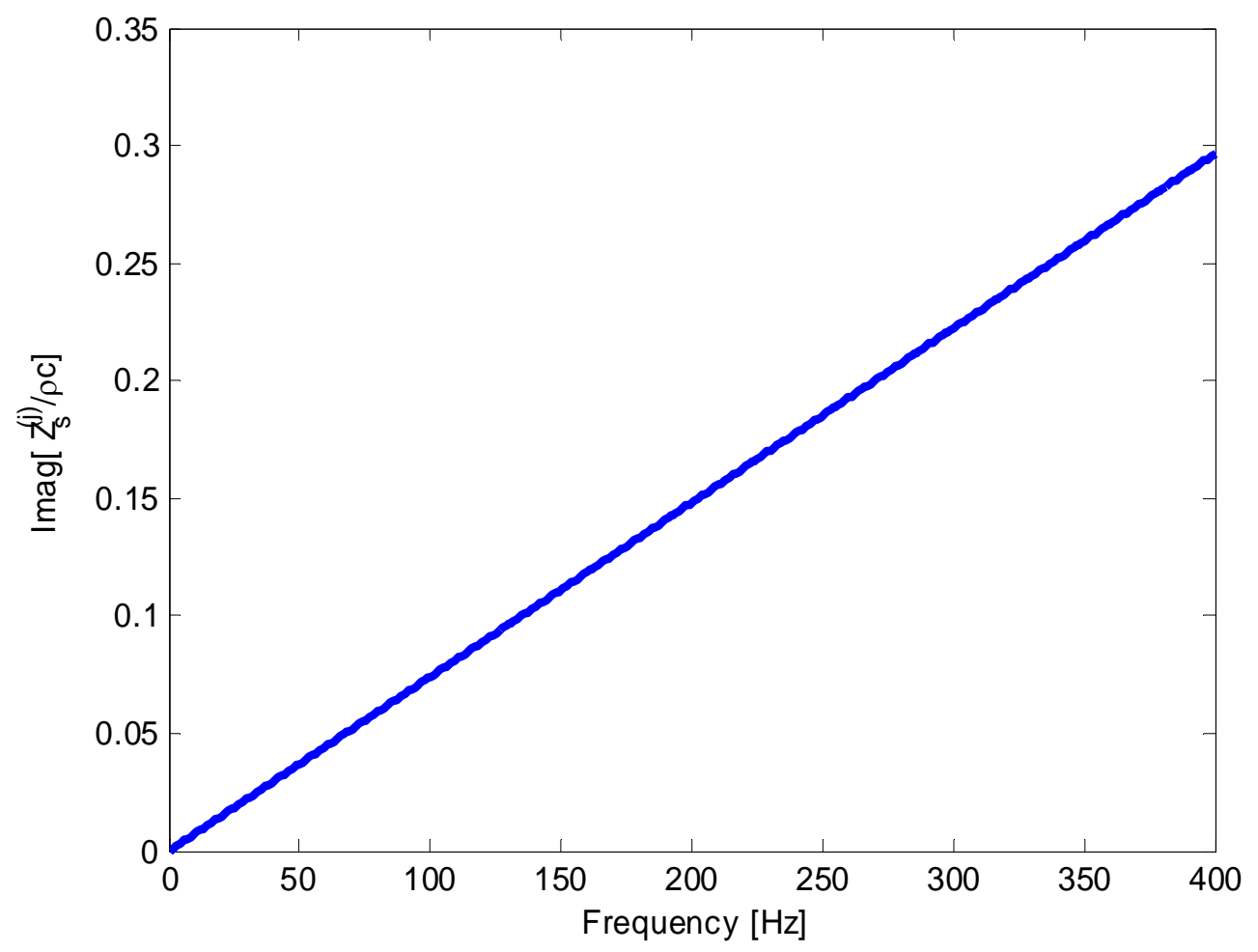

Figure F.2: Facesheet Normalized Impedance - Reactive Component 


\section{Appendix G: Solving The COUPLing MATRICES}

The acoustic pressure it written as:

$$
\begin{aligned}
p(x, \theta, \pi)= & \int_{0}^{2 \pi} \int_{0}^{L_{T}} G\left(x, \theta, \pi \mid x_{0}, \theta_{0}, \alpha\right) i \omega \rho \dot{w}\left(x_{0} \theta_{0}\right) a d x_{0} d \theta_{0} \\
& +\frac{i \omega \rho_{A}}{z_{11} z_{22}-z_{12} z_{21}}\left\{\int_{0}^{2 \pi} \int_{0}^{L_{T}} G\left(x_{0}^{(1)} \theta_{0}^{(1)}, b \mid x_{0} \theta_{0}, a\right) a \dot{w}\left(x_{0} \theta_{0}\right) d x_{0} d \theta_{0}\right. \\
& {\left[-S_{1} G\left(x, \theta, \pi \mid x_{0}^{(1)} \theta_{0}^{(1)}, b\right) z_{22}+S_{2} G\left(x, \theta, \pi \mid x_{0}^{(2)} \theta_{0}^{(2)}, b\right) z_{21}\right] } \\
& +\int_{0}^{2 \pi} \int_{0}^{L} G\left(x_{0}^{(2)} \theta_{0}^{(2)}, b \mid x_{0}, \theta_{0}, a\right) a \dot{w}\left(x_{0}, \theta_{0}\right) d x_{0} d \theta_{0} \\
& {\left[S_{1} G\left(x, \theta, \pi \mid x_{0}^{(1)} \theta_{0}^{(1)}, b\right) z_{12}+S_{2} G\left(x, \theta, \pi \mid x_{0}^{(2)} \theta_{0}^{(2)}, b\right) z_{11}\right] }
\end{aligned}
$$

Replacing (G.1) in the last equation, it yields then

$$
\begin{aligned}
& \int_{0}^{2 \pi} \int_{0}^{L_{T}}\left[\Phi_{s r}\right]^{T}\left\{\begin{array}{c}
0 \\
0 \\
p(x, \theta, a)
\end{array}\right\} a d \theta d x=\int_{0}^{2 \pi} \int_{0}^{L_{T}}\left\{\begin{array}{l}
W_{s r}^{(1)} \\
W_{s r}^{(2)} \\
W_{s r}^{(3)}
\end{array}\right\} \sin \left(\frac{s \pi}{L_{T}} x\right) \cos (r \theta)\left[\int_{0}^{2 \pi} \int_{0}^{L} G\left(x \theta, a \mid x_{0} \theta_{0}, a\right)\right. \\
& i \omega \rho\left[i \omega \sum_{m=1}^{M} \sum_{n=0}^{N}\left\{W_{m n}^{(1)}, W_{m n}^{(2)}, W_{m n}^{(3)}\right\}\left\{\begin{array}{l}
A_{m n}^{(1)} \\
A_{m n}^{(2)} \\
A_{m n}^{(3)}
\end{array}\right\} \sin \left(\frac{m \pi}{L_{T}} x_{0}\right) \cos \left(n \theta_{0}\right)\right] a d x_{0} d \theta_{0}+\frac{i \omega \rho}{\left(z_{11} z_{22}-z_{12} z_{21}\right)} . \\
& {\left[-S_{1} G\left(x, \theta, \pi \mid x_{0}^{(1)} \theta_{0}^{(1)}, b\right) z_{22}+S_{2} G\left(x, \theta, \pi \mid x_{0}^{(2)} \theta_{0}^{(2)}, b\right) z_{21}\right]+\int_{0}^{2 \pi} \int_{0}^{L_{T}} G\left(x_{0}^{(2)} \theta_{0}^{(2)}, b \mid x_{0}, \theta_{0}, a\right)} \\
& \left\{\int_{0}^{2 \pi} \int_{0}^{L_{T}} G\left(x_{0}^{(1)} \theta_{0}^{(1)}, b \mid x_{0}, \theta_{0}, a\right) a\left[i \omega \sum_{m=1}^{M} \sum_{n=0}^{N}\left\{W_{m n}^{(1)}, W_{m n}^{(2)}, W_{m n}^{(3)}\right\}\left\{\begin{array}{c}
A_{m n}^{(1)} \\
A_{m n}^{(2)} \\
A_{m n}^{(3)}
\end{array}\right\} \sin \left(\frac{m \pi}{L_{T}} x_{0}\right) \cos \left(n \theta_{0}\right)\right] d x_{0} d \theta_{0} .\right. \\
& a\left[i \omega \sum_{m=1}^{M} \sum_{n=0}^{N}\left\{W_{m n}^{(1)}, W_{m n}^{(2)}, W_{m n}^{(3)}\right\}\left\{\begin{array}{l}
A_{m n}^{(1)} \\
A_{m n}^{(2)} \\
A_{m n}^{(3)}
\end{array}\right\} \sin \left(\frac{n \pi}{L_{T}} x_{0}\right) \cos \left(n \theta_{0}\right)\right] d x_{0} d \theta_{0} \\
& {\left[S_{1} G\left(x, \theta, a \mid x_{0}^{(1)} \theta_{0}^{(1)}, b\right) z_{12}+S_{2} G\left(x, \theta, \pi \mid x_{0}^{(2)} \theta_{0}^{(2)}, b\right) z_{11}\right] a d x d \theta}
\end{aligned}
$$


Combining the terms, it yields

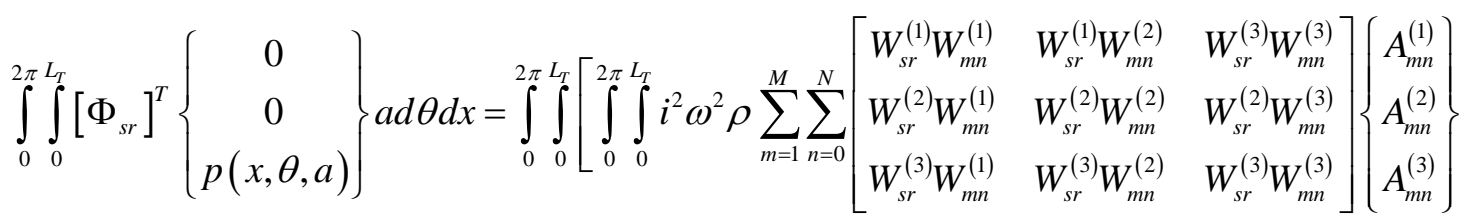

$$
\begin{aligned}
& \left.\sin \left(\frac{s \pi}{L_{T}} x\right) \cos (n \theta) \cdot G\left(x, \theta, a \mid x_{0} \theta_{0}, a\right) \sin \left(\frac{m \pi}{L_{T}} x_{0}\right) \cdot \cos \left(n \theta_{0}\right) a d x_{0} d \theta_{0}\right] a d x d \theta \\
& +\frac{i \omega \rho}{\left(z_{11} Z_{22}-Z_{12} Z_{21}\right)}\left\{\int _ { 0 } ^ { 2 \pi } \int _ { 0 } ^ { L _ { T } } \left[\int_{0}^{2 \pi} \int_{0}^{L_{T}} i \omega \sum_{m=1}^{M} \sum_{n=0}^{N}\left[\begin{array}{ccc}
W_{s r}^{(1)} W_{m n}^{(1)} & W_{s r}^{(1)} W_{m n}^{(2)} & W_{s r}^{(3)} W_{m n}^{(3)} \\
W_{s r}^{(2)} W_{m n}^{(1)} & W_{s r}^{(2)} W_{m n}^{(2)} & W_{s r}^{(2)} W_{m n}^{(3)} \\
W_{s r}^{(3)} W_{m n}^{(1)} & W_{s r}^{(3)} W_{m n}^{(2)} & W_{s r}^{(3)} W_{m n}^{(3)}
\end{array}\right]\left\{\begin{array}{c}
A_{m n}^{(1)} \\
A_{m n}^{(2)} \\
A_{m n}^{(3)}
\end{array}\right\}\right.\right. \\
& \left.G\left(x_{0}^{(1)} \theta_{0}^{(1)}, b \mid x_{0}, \theta_{0}, a\right) \sin \left(\frac{n \pi}{L_{T}} x_{0}\right) \cos \left(n \theta_{0}\right) a d x_{0} d \theta_{0}\right] \cdot\left[-S_{1} G\left(x, \theta, \pi \mid x_{0}^{(1)} \theta_{0}^{(1)}, b\right) .\right. \\
& \left.\sin \left(\frac{s \pi}{L_{T}} x_{0}\right) \cdot \cos \left(r \theta_{0}\right) z_{22}+S_{2} G\left(x, \theta, a \mid x_{0}^{(2)} \theta_{0}^{(2)}, b\right) \sin \left(\frac{s \pi}{L_{T}} x\right) \cdot \cos \left(r \theta_{0}\right) z_{21}\right] a d x d \theta+ \\
& +\int_{0}^{2 \pi} \int_{0}^{L_{T}}\left[\int_{0}^{2 \pi} \int_{0}^{L_{T}} i \omega \sum_{m=1}^{M} \sum_{n=0}^{N}\left[\begin{array}{ccc}
W_{s r}^{(1)} W_{m n}^{(1)} & W_{s r}^{(1)} W_{m n}^{(2)} & W_{s r}^{(3)} W_{m n}^{(3)} \\
W_{s r}^{(2)} W_{m n}^{(1)} & W_{s r}^{(2)} W_{m n}^{(2)} & W_{s r}^{(2)} W_{m n}^{(3)} \\
W_{s r}^{(3)} W_{m n}^{(1)} & W_{s r}^{(3)} W_{m n}^{(2)} & W_{s r}^{(3)} W_{m n}^{(3)}
\end{array}\right]\left\{\begin{array}{c}
A_{m n}^{(1)} \\
A_{m n}^{(2)} \\
A_{m n}^{(3)}
\end{array}\right\} \cdot G\left(x_{0}^{(2)} \theta_{0}^{(2)}, b \mid x_{0}, \theta_{0}, a\right)\right. \\
& \left.\cdot \sin \left(\frac{s \pi}{L_{T}} x_{0}\right) \cdot \cos \left(r \theta_{0}\right) a d x_{0} d \theta_{0}\right] \cdot\left[S_{1} G\left(x, \theta, a \mid x_{0}^{(1)} \theta_{0}^{(1)}, b\right) \sin \left(\frac{s \pi}{L_{T}} x\right) \cdot \cos (r \theta) z_{12}\right. \\
& \left.-S_{2} G\left(x, \theta, a \mid x_{0}^{(1)} \theta_{0}^{(1)}, b\right) \sin \left(\frac{s \pi}{L_{T}} x\right) \cdot \cos (r \theta) z_{11}\right] a d x d \theta
\end{aligned}
$$

The integrals are given by

$$
\begin{aligned}
& \int_{0}^{L_{T}} \sin \left(\frac{s \pi}{L_{T}} x\right) \cdot \cos \left(\frac{q \pi}{L_{T}} x\right) d x=\varepsilon_{s q}=\left\{\frac{L_{T} s\left[-1+(-1)^{q+s}\right]}{\pi\left(q^{2}-s^{2}\right)}, q \neq s\right. \\
& \int_{0}^{2 \pi} \cos (r \theta) \cdot \cos (p \theta) a d \theta=a \pi \delta_{r p} \varepsilon_{r}, \quad \text { with } \varepsilon_{p}= \begin{cases}2, & r=0 \\
1, & r \neq 0\end{cases}
\end{aligned}
$$


Where

$$
\int_{0}^{L_{T}} \sin \left(\frac{m \pi}{L_{T}} x\right) \cdot \cos \left(\frac{q \pi}{L_{T}} x_{0}\right) d x=\varepsilon_{m q}=\left\{\begin{array}{cl}
0, & q=m \\
\frac{L_{T} m}{\pi\left(q^{2}-n^{2}\right)}\left[-1+(-1)^{q+m}\right], & q \neq m
\end{array}\right.
$$

and

$$
\int_{0}^{2 \pi} \cos \left(n \theta_{0}\right) \cdot \cos \left(p \theta_{0}\right) a d \theta_{0}=a \pi \delta_{n p} \varepsilon_{n}, \quad \text { with } \varepsilon_{n}=\left\{\begin{array}{l}
2, n=0 \\
1, n \neq 0
\end{array}\right.
$$

Then

$$
\begin{aligned}
& \int_{0}^{2 \pi} \int_{0}^{L_{T}}\left[\Phi_{s r}\right]^{T}\left\{\begin{array}{c}
0 \\
0 \\
p(x, \theta, a)
\end{array}\right\} a d x d \theta=i^{2} \omega^{2} \rho \sum_{m=1}^{M} \sum_{n=0}^{N} \sum_{q=0}^{Q} \sum_{p=0}^{P} \sum_{l=0}^{L} \frac{\beta^{2}(a)}{\Lambda_{q p \rho}\left(k_{q p \rho}^{2}-k^{2}\right)} \\
& {\left[\begin{array}{lll}
W_{s r}^{(1)} W_{m n}^{(1)} & W_{s r}^{(1)} W_{m n}^{(2)} & W_{s r}^{(3)} W_{m n}^{(3)} \\
W_{s r}^{(2)} W_{m n}^{(1)} & W_{s r}^{(2)} W_{m n}^{(2)} & W_{s r}^{(2)} W_{m n}^{(3)} \\
W_{s r}^{(3)} W_{m n}^{(1)} & W_{s r}^{(3)} W_{m n}^{(2)} & W_{s r}^{(3)} W_{m n}^{(3)}
\end{array}\right]\left\{\begin{array}{c}
A_{m n}^{(1)} \\
A_{m n}^{(2)} \\
A_{m n}^{(3)}
\end{array}\right\}\left(\varepsilon_{s q} \cdot a \pi \delta_{n p} \varepsilon_{n}\right)+\frac{i \omega \rho_{A}}{\left(z_{11} z_{22}-z_{12} z_{21}\right)}} \\
& \left\{i \omega \sum_{m=1}^{M} \sum_{n=0}^{N} \sum_{q=0}^{Q} \sum_{p=0}^{P} \sum_{l=0}^{L} \frac{\beta(b) \beta(a)}{\Lambda_{q p \rho}\left(k_{q p \rho}^{2}-k^{2}\right)} \cdot \cos \left(q \theta_{0}^{(1)}\right) \cos \left(\frac{q \pi}{L_{T}} x_{0}^{(1)}\right)\left(\varepsilon_{m q} a \pi \delta_{n p} \varepsilon_{n}\right)\right. \\
& {\left[\begin{array}{ccc}
W_{s r}^{(1)} W_{m n}^{(1)} & W_{s r}^{(1)} W_{m n}^{(2)} & W_{s r}^{(3)} W_{m n}^{(3)} \\
W_{s r}^{(2)} W_{m n}^{(1)} & W_{s r}^{(2)} W_{m n}^{(2)} & W_{s r}^{(2)} W_{m n}^{(3)} \\
W_{s r}^{(3)} W_{m n}^{(1)} & W_{s r}^{(3)} W_{m n}^{(2)} & W_{s r}^{(3)} W_{m n}^{(3)}
\end{array}\right]\left\{\begin{array}{c}
A_{m n}^{(1)} \\
A_{m n}^{(2)} \\
A_{m n}^{(3)}
\end{array}\right\}} \\
& {\left[-S_{1} \sum_{q=0}^{Q} \sum_{p=0}^{P} \sum_{l=0}^{L} \frac{\beta(b) \beta(a)}{\Lambda_{q p l}\left(k_{q p l}^{2}-k^{2}\right)} \cos \left(\frac{q \pi}{L_{T}} x_{0}^{(1)}\right) \cos \left(p \theta_{0}^{(1)}\right) \cdot\left(\varepsilon_{s q} a \pi \delta_{r p} \varepsilon_{r}\right) z_{22}\right.} \\
& \left.+S_{2} \sum_{q=0}^{Q} \sum_{p=0}^{P} \sum_{l=0}^{L} \frac{\beta(b) \beta(a)}{\Lambda_{q p l}\left(k_{q p l}^{2}-k^{2}\right)} \cos \left(\frac{q \pi}{L_{T}} x_{0}^{(2)}\right) \cos \left(p \theta_{0}^{(2)}\right) \cdot\left(\varepsilon_{s q} a \pi \delta_{r p} \varepsilon_{r}\right) z_{21}\right] \\
& +i \omega \sum_{m=1}^{M} \sum_{n=0}^{N} \sum_{q=0}^{Q} \sum_{p=0}^{P} \sum_{l=0}^{L} \frac{\beta(b) \beta(a)}{\Lambda_{q p \rho}\left(k_{q p \rho}^{2}-k^{2}\right)} \cdot \cos \left(q \theta_{0}^{(2)}\right) \cdot \cos \left(\frac{q \pi}{L_{T}} x_{0}^{(2)}\right)\left(\varepsilon_{m q} a \pi \delta_{n p} \varepsilon_{n}\right) \text {. } \\
& {\left[\begin{array}{ccc}
W_{s r}^{(1)} W_{m n}^{(1)} & W_{s r}^{(1)} W_{m n}^{(2)} & W_{s r}^{(3)} W_{m n}^{(3)} \\
W_{s r}^{(2)} W_{m n}^{(1)} & W_{s r}^{(2)} W_{m n}^{(2)} & W_{s r}^{(2)} W_{m n}^{(3)} \\
W_{s r}^{(3)} W_{m n}^{(1)} & W_{s r}^{(3)} W_{m n}^{(2)} & W_{s r}^{(3)} W_{m n}^{(3)}
\end{array}\right]\left\{\begin{array}{c}
A_{m n}^{(1)} \\
A_{m n}^{(2)} \\
A_{m n}^{(3)}
\end{array}\right\}} \\
& {\left[S_{1} \sum_{q=0}^{Q} \sum_{p=0}^{P} \sum_{l=0}^{L} \frac{\beta(b) \beta(a)}{\Lambda_{q p l}\left(k_{q p l}^{2}-k^{2}\right)} \cos \left(\frac{q \pi}{L_{T}} x_{0}^{(1)}\right) \cos \left(p \theta_{0}^{(1)}\right) \cdot\left(\varepsilon_{s q} a \pi \delta_{r p} \varepsilon_{r}\right) z_{12}\right.} \\
& \left.\left.-S_{2} \sum_{q=0}^{Q} \sum_{p=0}^{P} \sum_{l=0}^{L} \frac{\beta(b) \beta(a)}{\Lambda_{q p l}\left(k_{q p l}^{2}-k^{2}\right)} \cos \left(\frac{q \pi}{L_{T}} x_{0}^{(2)}\right) \cos \left(p \theta_{0}^{(2)}\right)\left(\varepsilon_{s q} a \pi \delta_{r p} \varepsilon_{r}\right) z_{11}\right]\right\}
\end{aligned}
$$


Therefore, the coupling matrices are given by

$$
\begin{aligned}
\sum_{q=0}^{Q} \sum_{p=0}^{P} \sum_{l=0}^{L}\left[\alpha_{\text {srmnqpl }}\right] & =-\frac{\rho}{\rho_{S}} \sum_{q=0}^{Q} \sum_{p=0}^{P} \sum_{l=0}^{L} \frac{\beta^{2}(a)}{\Lambda_{q p l}\left(k_{q p l}^{2}-k^{2}\right)} \\
& \delta_{r p} \delta_{n p} \varepsilon_{r} \varepsilon_{n} \varepsilon_{s q} \varepsilon_{m q} a^{2} \pi^{2}\left[\begin{array}{lll}
W_{s r}^{(1)} W_{m n}^{(1)} & W_{s r}^{(1)} W_{m n}^{(2)} & W_{s r}^{(3)} W_{m n}^{(3)} \\
W_{s r}^{(2)} W_{m n}^{(1)} & W_{s r}^{(2)} W_{m n}^{(2)} & W_{s r}^{(2)} W_{m n}^{(3)} \\
W_{s r}^{(3)} W_{m n}^{(1)} & W_{s r}^{(3)} W_{m n}^{(2)} & W_{s r}^{(3)} W_{m n}^{(3)}
\end{array}\right]
\end{aligned}
$$

and

$$
\begin{aligned}
& \sum_{q=0}^{Q} \sum_{p=0}^{P} \sum_{l=0}^{L}\left[\alpha_{\text {srmnqpl }}^{(1)}\right]=-\frac{\rho}{\rho_{S}\left(z_{11} z_{22}-z_{12} z_{21}\right)} \sum_{q=0}^{Q} \sum_{p=0}^{P} \sum_{l=0}^{L} \frac{\beta(b) \beta(a)}{\Lambda_{q p l}\left(k_{q p l}^{2}-k^{2}\right)} \cos \left(\frac{q \pi}{L_{T}} x_{0}^{(2)}\right) \cos \left(p \theta_{0}^{(2)}\right) \\
& \left(\varepsilon_{m q} a \pi \delta_{n p} \varepsilon_{n}\right)\left[\begin{array}{ccc}
W_{s r}^{(1)} W_{m n}^{(1)} & W_{s r}^{(1)} W_{m n}^{(2)} & W_{s r}^{(3)} W_{m n}^{(3)} \\
W_{s r}^{(2)} W_{m n}^{(1)} & W_{s r}^{(2)} W_{m n}^{(2)} & W_{s r}^{(2)} W_{m n}^{(3)} \\
W_{s r}^{(3)} W_{m n}^{(1)} & W_{s r}^{(3)} W_{m n}^{(2)} & W_{s r}^{(3)} W_{m n}^{(3)}
\end{array}\right] \\
& {\left[S_{1} \sum_{q=0}^{Q} \sum_{p=0}^{P} \sum_{l=0}^{L} \frac{\beta(b) \beta(a)}{\Lambda_{q p l}\left(k_{q p l}^{2}-k^{2}\right)} \cos \left(\frac{q \pi}{L_{T}} x_{0}^{(1)}\right) \cos \left(p \theta_{0}^{(1)}\right) \varepsilon_{s q} a \pi \delta_{r p} \varepsilon_{r} z_{12}-\right.} \\
& \left.-S_{1} \sum_{q=0}^{Q} \sum_{p=0}^{P} \sum_{l=0}^{L} \frac{\beta(a) \beta(b)}{\Lambda_{q p l}\left(k_{q p l}^{2}-k^{2}\right)} \cos \left(\frac{q \pi}{L_{T}} x_{0}^{(2)}\right) \cos \left(p \theta_{0}^{(2)}\right) \varepsilon_{s q} a \pi \delta_{r p} \varepsilon_{r} z_{11}\right]
\end{aligned}
$$

and

$$
\begin{aligned}
\sum_{q=0}^{Q} \sum_{p=0}^{P} \sum_{l=0}^{L}\left[\alpha_{\text {srmnqpl }}^{(2)}\right]= & -\frac{\rho}{\rho_{S}\left(z_{11} z_{22}-z_{12} z_{21}\right)} \sum_{q=0}^{Q} \sum_{p=0}^{P} \sum_{l=0}^{L} \frac{\beta(b) \beta(a)}{\Lambda_{q p l}\left(k_{q p l}^{2}-k^{2}\right)} \cos \left(\frac{q \pi}{L_{T}} x_{0}^{(1)}\right) \cos \left(p \theta_{0}^{(1)}\right) \\
& \left(\varepsilon_{m q} a \pi \delta_{n p} \varepsilon_{n}\right)\left[\begin{array}{lll}
W_{s r}^{(1)} W_{m n}^{(1)} & W_{s r}^{(1)} W_{m n}^{(2)} & W_{s r}^{(3)} W_{m n}^{(3)} \\
W_{s r}^{(2)} W_{m n}^{(1)} & W_{s r}^{(2)} W_{m n}^{(2)} & W_{s r}^{(2)} W_{m n}^{(3)} \\
W_{s r}^{(3)} W_{m n}^{(1)} & W_{s r}^{(3)} W_{m n}^{(2)} & W_{s r}^{(3)} W_{m n}^{(3)}
\end{array}\right] \\
& {\left[-S_{1} \sum_{q=0}^{Q} \sum_{p=0}^{P} \sum_{l=0}^{L} \frac{\beta(a) \beta(b)}{\Lambda_{q p l}\left(k_{q p l}^{2}-k^{2}\right)} \cos \left(\frac{q \pi}{L_{T}} x_{0}^{(1)}\right) \cos \left(p \theta_{0}^{(1)}\right)\left(\varepsilon_{s q} a \pi \delta_{r p} \varepsilon_{r}\right) z_{22}\right.} \\
& \left.+S_{2} \sum_{q=0}^{Q} \sum_{p=0}^{P} \sum_{l=0}^{L} \frac{\beta(a) \beta(b)}{\Lambda_{q p l}\left(k_{q p l}^{2}-k^{2}\right)} \cos \left(\frac{q \pi}{L_{T}} x_{0}^{(2)}\right) \cos \left(p \theta_{0}^{(2)}\right)\left(\varepsilon_{s q} a \pi \delta_{r p} \varepsilon_{r}\right) z_{21}\right]
\end{aligned}
$$


The symmetry by pairs is an important property of these matrices. This property is used to solve the coupled linear system of equation for the unknown modal amplitudes presented in Chapter 4 . The symmetry by pairs is given by

$$
\begin{aligned}
& \sum_{q=0}^{Q} \sum_{p=0}^{P} \sum_{l=0}^{L}\left[\alpha_{\text {srmnqpl }}\right]=\sum_{q=0}^{Q} \sum_{p=0}^{P} \sum_{l=0}^{L}\left[\alpha_{\text {mnsrapl }}\right] \\
& \sum_{q=0}^{Q} \sum_{p=0}^{P} \sum_{l=0}^{L}\left[\alpha_{\text {srmnqpl }}^{(1)}\right]=\sum_{q=0}^{Q} \sum_{p=0}^{P} \sum_{l=0}^{L}\left[\alpha_{\text {mnsrapl }}^{(1)}\right] \\
& \sum_{q=0}^{Q} \sum_{p=0}^{P} \sum_{l=0}^{L}\left[\alpha_{\text {srmnqpl }}^{(2)}\right]=\sum_{q=0}^{Q} \sum_{p=0}^{P} \sum_{l=0}^{L}\left[\alpha_{\text {mnsrapl }}^{(2)}\right]
\end{aligned}
$$




\section{VITA}

Leonardo Molisani was born in April $12^{\text {th }}$ of 1973 , in Alejo Ledesma, Córdoba, Argentina. After completion of high school in 1991, Leonardo attended to the National University of Río Cuarto, where he completed a Bachelor of Science degree in Mechanical Engineering. While at the university he was part of a research team in the Mechanical Department working in a heat transfer project related to a radiation problem in a micro satellite. Subsequent to his graduation in 1997, the author worked as teacher and researcher in areas of structural vibrations and computational mechanics at the National University of Río Cuarto. In Spring of 2000, he began graduates studies in the Mechanical Department at the Virginia Polytechnic Institute \& State University. During the completion of his graduate program he investigated in the area of vibration and acoustics. 